Universidade de São Paulo

Instituto de Astronomia, Geofísica e Ciências Atmosféricas

Departamento de Astronomia

Ulisses Diego A. S. Machado

\title{
Relações de Dispersão Deformadas na Cosmologia Inflacionária
}

\author{
São Paulo
}

2012

Versão corrigida. A versão original encontra-se disponivel na Unidade. 



\author{
Ulisses Diego A. S. Machado
}

\title{
Relações de Dispersão Deformadas na Cosmologia Inflacionária
}

Tese apresentada ao Departamento de Astronomia do Instituto de Astronomia, Geofísica e Ciências Atmosféricas da Universidade de São Paulo como requisito parcial para a obtenção do título de Doutor em Ciências.

Área de Concentração: Astronomia Orientador: Prof. Dr. Reuven Opher

\author{
São Paulo
}

2012

Versão corrigida. A versão original encontra-se disponivel na Unidade. 

Dedico esta tese a quem acreditou em mim...E a quem lê-la, que possa sentir em cada palavra nela escrita que não houve um único dia que eu não tenha dado o melhor de $\operatorname{mim} .$. 



\section{Agradecimentos}

Eu acredito que o doutorado é uma fase muito importante no surgimento de um novo pesquisador. Quando eu comecei o meu doutorado, logo percebi que a minha então incipiente postura científica e interesses eram diferentes das do meu orientador R. Opher e até mesmo do instituto de Astronomia. Apesar de tudo, R. Opher me deu liberdade para seguir o caminho que eu desejava e me tornar o tipo de pesquisador que eu queria ser. Foi um caminho muito difícil em que muitas pessoas duvidaram que eu pudesse ter êxito, mas mesmo assim eu perseverei, porque eu acreditava que era o que eu deveria fazer. Shakespeare dizia que se leva muito tempo para se tornar a pessoa que se quer ser e o tempo é curto. Eu senti a veracidade dessas palavras nesses anos. Apesar de tudo, devo agradecer ao meu orientador pela oportunidade de poder seguir o que eu queria fazer e tentar me tornar aquilo que eu sinto que eu deveria ser. Continuarei nesta busca, por muito além do fim deste doutorado...

Eu agradeço aos meus amigos, principalmente, Douglas Barros, que assim como eu, veio do DF UFPE, onde infelizmente não tivemos a oportunidade de nos conhecermos, mas nos conhecemos aqui e logo nos tornamos grandes amigos. Ele sempre me ouviu nos momentos difíceis em que eu precisava ser ouvido.

Agradeço ao Departamento de Física da UFPE. Uma excelente instituição a que devo muito do que eu sei hoje. Não fosse pela excelente formação dada a mim por esta instituição, eu não teria tido a menor chance de superar os obstáculos que eu enfrentei aqui.

Agradeço ao CNPQ pelo auxílio financeiro através do qual eu pude ter acesso a tudo que eu precisei para tornar o projeto factível.

Agradeço aos meus amigos cosmólogos, alunos do Prof. R. Opher e do prof. Ademir, pois compartilhamos esse momento tão importante de nossas vidas e muitas vezes nos 
ajudamos, nem que fosse com palavras amigas.

Gostaria particularmente de agradecer ao meu amigo Rafael que, sendo mais experiente e de competência indiscutível, ajudou, quando não com valiosas colaborações, com ensinamentos valiosos sobre a arte de fazer ciência. Sem essa ajuda, tudo teria sido muito mais difícil.

Agradeço ao professor Ademir, pois apesar de não ser meu orientador, nas poucas oportunidades em que pude usufruir da sua imensa sabedoria, aprendi coisas muito importantes.

Agradeço ao prof. Marcelo Gomes que me ensinou as primeiras lições sobre QFT e deu ajudas fundamentais sobre teorias não comutativas. Apesar de ele talvez achar que não fez muito por mim, cada minuto que eu o ouvi, fez uma grande diferença pra mim. Isso se estende ao seu grupo de pesquisa.

Eu gostaria de agradecer especialmente à Karina Guirado, que foi minha namorada e amiga, e que me acompanhou por todos esses anos. Que teve fé em mim até nos momentos em que eu mesmo tinha perdido. Que com sua (por vezes) imensa sabedoria de vida me reanimou até nos momentos em que eu não via sentido em continuar. Quando minha força interior faltou, ela me emprestou a dela.

Por fim, mas não menos importante, eu gostaria de agradecer à minha mãe, Lisieux de Maria, pois sem o apoio dela eu não seria ninguém. Todos os momentos em que eu precisei de qualquer tipo de ajuda-e como eu precisei- ela sempre esteve pronta para ajudar. Desde o momento em que eu decidi seguir a caminho da ciência, aos quatorze anos, ela me incentivou. Eu não teria chegado até aqui sem o apoio dela... Nós sempre estivemos longe do resto da nossa família... Cabia a cada um de nós ser a família do outro... 
Durante toda a minha vida, eu quis acreditar que trazia comigo algo especial, um talento ou algo assim que me faria vencer todos os obstáculos que se interpusessem no meu caminho. Mas cada vez que eu fracassava, sentia o meu orgulho ferido e minha confiança em mim mesmo abalada. As dificuldades me faziam retroceder em dúvida. Hoje, acredito em algo inabalável: que levo sim comigo algo raro e especial, não talento, mas algo muito mais raro chamado propósito. Por acreditar nisso, não mais retrocedo ou hesito, as vezes que caio não me desmerecem, pois estimo o meu valor não pelo número de vezes que evito a queda, mas sim pelo número de vezes que sou capaz de tornar a me levantar.

Valemos pelo sol que buscamos e não pela sombra que deixamos para trás...

"Se a vida tem mil ondas como o mar, assim como as ondas do mar, devemos fazer de cada recuo o caminho para um avanço ainda maior"

Lisieux de Maria (Minha mãe)

" This is my quest

To follow that star No matter how hopeless

No matter how far 



\section{Resumo}

Relação de dispersão é outro nome para a função Hamiltoniana, cujo conhecimento especifica completamente a dinâmica de um sistema no formalismo da mecânica clássica. Sua escolha está intimamente vinculada às simetrias do sistema e, no contexto cosmológico aqui apresentado, com as simetrias locais obedecidas pelas leis físicas. Mais ainda, a contribuição da matéria na dinâmica cosmológica reflete a escolha do grupo local de simetrias das leis físicas.

Por outro lado, o problema fundamental da cosmologia pode ser definido como a construção de um modelo de evolução temporal de estados que, sob as hipóteses mais simples sobre estados iniciais (digamos, que demande a menor quantidade de informação possível para serem enunciadas), prediga o estado atual observado. O paradigma inflacionário é atualmente a ideia que melhor cumpre esta definição, uma vez que prediz que uma grande variedade de condições iniciais leva a aspectos fundamentais do universo observado. Contudo, os mecanismos usuais de realização da inflação sofrem de problemas conceituais. O ponto de vista deste trabalho é que a realização convencional da inflação, isto é, através dos campos escalares minimamente acoplados, é a formulação localmente relativisticamente invariante da inflação. A maneira de incluir quebras e deformações da estrutura de simetrias locais na cosmologia é não única e está associado ao chamado problema trans Planckiano da inflação. Analogamente, a motivação conceitual para incluir esse tipo de modificação tampouco é única. Dependendo do esquema de realização, a versão localmente não relativística da mesma pode apresentar graves dificuldades de conciliação com observações atuais, ou apresentar vantagens conceituais em relação ao modelo padrão de inflação, enquanto em conformidade com observações cosmológicas.

Da maneira como foi posto o problema fundamental da cosmologia, a escolha das 
simetrias locais influi na regra de evolução dos estados. O conceito de simetrias encontra sua formulação independente de teorias físicas no formalismo da teoria de grupos, mas consideraremos uma extensão da ideia, de aplicabilidade mais geral, a teoria das álgebras de Hopf que, de certo modo, trata das simetrias de estruturas algébricas. Esta extensão é útil inclusive no trato de simetrias dos espaços não comutativos, uma das principais propostas físicas que em última análise afeta a estrutura de simetrias locais do espaçotempo.

A expressão "simetrias locais", por si só, não diz muito sem a consideração de regras de realização. Essas regras dependem da estrutura matemática das observáveis da teoria. Sob hipóteses muito gerais, que não especificam uma teoria em particular, é possível mostrar, não como um teorema matemático formal, mas como uma hipótese tecnicamente bem motivada, que existem apenas dois tipos de teorias físicas: as clássicas e as quânticas. Trabalharemos sob essas hipóteses, as quais se formulam algebricamente assumindo a estrutura de $C^{*}$-álgebra para as observáveis físicas, outra motivação para o uso das álgebras de Hopf para descrição das simetrias da natureza. 


\section{Abstract}

Dispersion relation is another name for the Hamiltonian function whose knowledge completely specifies the dynamics in the formalism of classical mechanics. Its choice is intimately related to the symmetries of the system, and, in the cosmological context here exposed, with the local space-time symmetries obeyed by physical laws.

For the other side, the fundamental problem of cosmology can be defined as a construction of a time evolution model of states which, under simplest possible hypothesis concerning initial conditions (say, which demands the minimal amount of information to be specified), predicts the present observed state. The inflationary paradigm is currently the idea which better accomplishes this definition, since it predicts that a great variety of initial conditions lead to essential aspects of observed universe. The usual mechanisms of inflation suffer, however, from conceptual problems. The point of view of this work is that the usual realization of inflation based on weakly coupled scalar fields suffer is the local relativistic invariant realization. The way of including breaks and deformations of local space-time symmetries is not unique and it is associated to the so called Trans-Planckian problem of inflation. Analogously, the motivation to include this kind of modification is neither unique. Depending of the scheme of realization, the locally non-relativistic version may lead to serious difficulties in conciliation with observations, or to conceptual advantages over standard formulations while in accordance with observational data.

In the way that was proposed the fundamental problem of cosmology, the choice of local symmetries affects the rule of evolution of states. The concept of symmetry finds its formulation independently of physical theories in the group theory formalism, but we will consider an extension of idea, of wider applicability, the theory of Hopf algebras, which, is about symmetries of algebraic structures. That extension is useful including dealing with 
symmetries of non-commutative spaces, one of the main physical proposals that, in de end, affect the structure of space-time symmetries.

The expression, " local symmetries", by itself, does not say too much without considering realization rules. Those rules depend on mathematical structure of observables in the theory. Under very general hypothesis that do not specify a particular theory, it is possible to show, not as a formal mathematical theorem, but as a technically well motivated hypothesis, that only two types of physical theories do exist: The classical ones and the quantum ones. We are going to work under those hypothesis, which can be algebraically formulated assuming a $C^{*}$-algebra structure for physical observables, another motivation for the use of algebraic structures like Hopf algebras for the description of nature symmetries. 


\section{Lista de Figuras}

$8.1 \quad$ Generic graphic of $w(T)$ versus $T . \ldots \ldots \ldots$

\begin{tabular}{lll|l|l}
\hline 8.2 & $\mathrm{w}(\mathrm{T})$ versus $\ln (\mathrm{T})$ para a função $g$ da Eq. & 8.4. & Temos nesse exemplo uma \\
\hline
\end{tabular} equação de estado de alta temperatura no intervalo inflacionário $(w(T \rightarrow$

$\infty) \approx-0.6)$, mas para algum $T$, temos que $w(T)<-1$, levando a um problema de saída graciosa. . . . . . . . . . . . . . . . . . . . . 195 



\section{Sumário}

1. Introdução . . . . . . . . . . . . . . . . . . . . . . . . 19

2. A estrutura clássica do espaço-tempo e matéria . . . . . . . . . . . . . . . . . 27

2.1 Introdução $\ldots \ldots \ldots \ldots \ldots \ldots$. . . . . . . . . . . . . . . . . . . . 27

2.2 O espaço-tempo plano $\ldots \ldots \ldots \ldots \ldots$

2.3 O espaço-tempo curvo . . . . . . . . . . . . . . . . . . . . . . . . 33

$2.3 .1 \quad$ A localidade na descrição da física . . . . . . . . . . . . . . . 33

$2.3 .2 \quad$ A formulação tensorial . . . . . . . . . . . . . . . . . . . . . 38

$2.3 .3 \quad$ As equações de campo . . . . . . . . . . . . . . . . . . . . . . . 45

3. Cosmologia inflacionária $\ldots \ldots \ldots \ldots \ldots$

3.1 Introdução . . . . . . . . . . . . . . . . . . . . . . . . . 53

$3.2 \quad$ A cosmologia de Friedmann-Robertson-Walker e seus problemas conceituais 54

$3.3 \quad$ O mecanismo de inflação dirigida por campo escalar . . . . . . . . . . . . . 62

$3.3 .1 \quad$ O rolamento lento . . . . . . . . . . . . . . . . . . . . . . . . 62

$3.3 .2 \quad$ Fim da inflação . . . . . . . . . . . . . . . . . . . . . . 66

4. Teoria de Perturbações cosmológicas . . . . . . . . . . . . . . . . . . . . . 71

4.1 Introdução . . . . . . . . . . . . . . . . . . . . . . . . . 71

$4.2 \quad$ A descrição estatística da matéria . . . . . . . . . . . . . . . . . 72

4.3 O problema de calibre das perturbações $\ldots \ldots \ldots \ldots$

4.4 Geração de perturbações . . . . . . . . . . . . . . . . . . . . . . . 82

$4.4 .1 \quad$ A evolução clássica das perturbações durante a inflação . . . . . . . . 82 
$4.4 .2 \quad$ A teoria quântica de perturbações cosmológicas . . . . . . . . . . . 86

5. O significado conceitual da relação de dispersão . . . . . . . . . . . . . . . . . . . . 95

$5.1 \quad$ Introdução . . . . . . . . . . . . . . . . . . . . . . . . . . . . . . . . . 95

$5.2 \quad$ O problema da realização de simetrias no contexto quântico . . . . . . . . 97

$5.2 .1 \quad$ O teorema de Wigner . . . . . . . . . . . . . . . . . . . 97

$5.2 .2 \quad$ A representação de grupos de Lie . . . . . . . . . . . . . . . . . 104

$5.2 .3 \quad$ A representação do Grupo de Poincaré . . . . . . . . . . . . . . . . 110

6. Espaços não comutativos, teoria quântica e a simetria relativística . . . . . . . . 131

$6.1 \quad$ Introdução . . . . . . . . . . . . . . . . . . . . . . . 131

$6.2 \quad$ A abordagem das álgebras $C^{*}$ para a teoria quântica $\ldots . . . . .132$

6.3 Teoria quântica sob hipótese de espaço-tempo quântico . . . . . . . . . . . 144

6.4 O caráter não relativístico de um espaço-tempo quântico . . . . . . . . . . 151

7. A Inflação Algébrica I:

A estrutura algébrica admissível e uma primeira formulação para a física infla-

cionária não relativística . . . . . . . . . . . . . . . . . . . . . . . . . . 163

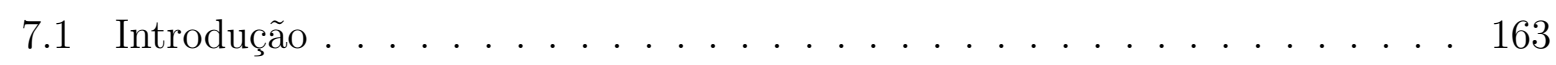

7.2 A abordagem da teoria de grupos para a inflação . . . . . . . . . . . . . 164

7.3 Estrutura algébrica admissível $\ldots$. . . . . . . . . . . . . . . . . . . . . . . 172

7.4 Sobre a conjectura da tradução da não comutatividade na estrutura algébrica

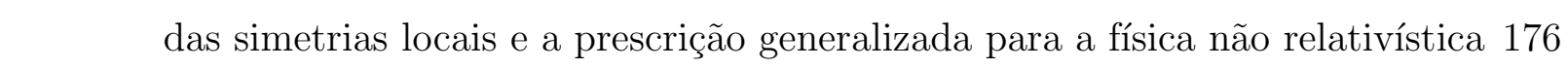
7.4 .1 As Hopf álgebras . . . . . . . . . . . . . . . . . . 176

7.4.2 Uma prescrição alternativa para a física quântica não relativística e/ou no espaço não comutativo: a prescrição $\pi_{\mathfrak{g}} \ldots$. . . . . . . . . . 182

8. A Inflação Algébrica II:

Uma nova prescrição para definir a física inflacionária não relativística. . . . . . . 189

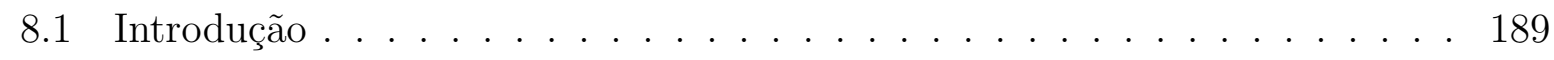

8.2 Condições sobre $f(E)$ análogas às condições de rolamento lento da inflação dirigida por campo escalar . . . . . . . . . . . . . . . . 190

8.2.1 Condição de termodinâmica aceitável e limite de baixas energias . . 191 
$8.2 .2 \quad$ Condição de número mínimo de e-folds . . . . . . . . . . . . . . . . 192

$8.2 .3 \quad$ A condição de saída graciosa . . . . . . . . . . . . . . . . . . . . . . 194

$8.3 \quad$ O algoritmo numérico . . . . . . . . . . . . . . . . . . . . . 196

8.4 Uma prescrição alternativa para a física quântica não relativística e/ou no espaço não comutativo: a prescrição $\pi_{\mathcal{A}} \ldots$. . . . . . . . . . . . . . . 198

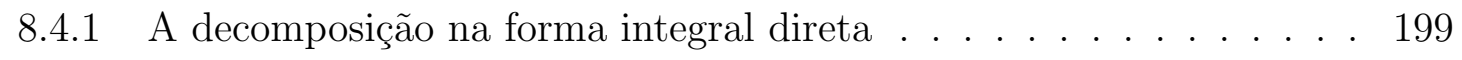

8.4.1.1 A decomposição integral direta da representação de uma álgebra no espaço de Hilbert. . . . . . . . . . . . . . . . . 202

8.4.2 A não existência de um grupo de Lie que realiza a inflação não comutativa . . . . . . . . . . . . . . . . . . 204

$8.4 .3 \quad$ A prescrição $\pi_{\mathcal{A}} \ldots \ldots \ldots \ldots$. . . . . . . . . . . . . 207

$8.5 \quad$ A existência da estrutura algébrica admissível . . . . . . . . . . . . . . . . 211

9. Conclusões . . . . . . . . . . . . . . . . . . . . . . . . . 213

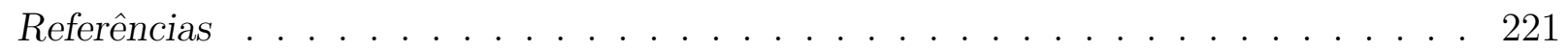



Capítulo 1

\section{Introdução}

Talvez possamos definir o problema fundamental da física como a construção um modelo de evolução temporal de estados em que cada um destes contém a informação de todas as possíveis observáveis a princípio, e no qual o estado num determinado instante qualquer é função unicamente determinada daquele num fixo instante arbitrário. Esse modelo de teoria física foi certamente enfraquecido pelo advento da teoria quântica e da relatividade geral. Nesta, porque é sabido que o chamado problema de Cauchy para equações de campo de Einstein só pode ser formulado em uma classe particular de espaço-tempo chamada de globalmente hiperbólica. Mas principalmente naquela, porque a teoria quântica prediz que não existe informação suficiente em nenhum instante do tempo para especificar completamente o futuro ou o passado 17. Mas de todo modo, mesmo em uma versão enfraquecida, num sentido matemático paradoxal em que um conceito anterior é substituído por outro mais abrangente, esse modelo de evolução temporal de estados está na base de toda a física. Sendo assim, a cosmologia distingue-se do resto da física pela inclusão de um elemento adicional no problema: a construção de um modelo de evolução temporal de estados que, sob as hipóteses mais simples sobre as condições do universo em algum momento suficientemente remoto do tempo, prediga o estado atual observado. Por mais simples, poderíamos definir a que demande a menor quantidade possível de informação para se especificar. Ao descrever tal estado, teoria e observação se confundem.

Se por um lado a informação que nos chega aos instrumentos e sentidos é essencialmente uma imagem produzida por fontes extraterrestres, a maneira como codificamos essa

\footnotetext{
${ }^{1}$ Além do mais, restringir a classe de espaço-tempo que ocorre na física não é diferente de restringir a classe de potenciais que ocorre na física Newtoniana de modo a evitar aqueles que violam as condições de unicidade de soluções dadas condições iniciais.
} 
informação em nosso modelo de universo é dependente da formulação das nossas teorias físicas. Na descrição clássica, a informação sobre o universo é completamente especificada pela escolha de uma variedade topológica diferenciável no qual se define certo conjunto de campos tensoriais que especificam a informação sobre a matéria e forças fundamentais como a gravidade. A finitude da velocidade máxima de propagação dos sinais no universo, sendo uma das ideias mais fundamentais na física do ponto de vista teórico e experimental, nos leva a uma conclusão de notável importância: a imagem que chega até nós é na verdade uma amostragem da história do universo. Não apenas, a noção de causalidade relativística, associada à existência desse limite, é de fundamental importância à cosmologia e afetado por considerações sobre simetrias fundamentais.

Em 1963, segundo Malcolm Longair (1993, QJRAS, 34, 157), Peter Scheuer teria dito:

"There are only two and a half facts in cosmology:

1. The sky is dark at night;

2. The galaxies are receding from each other as expected in a uniform expansion;

3. The contents of the Universe have probably changed as the Universe grows older;" Outra famosa frase, atribuída a Landau, é:

"Cosmologists are often in error but never in doubt."

Se não pudermos dizer que a descoberta da expansão do universo por Hubble em 1929 elevou a cosmologia ao status de ciência pela falseabilidade de suas previsões, então esse status é inescapável à descoberta da radiação cósmica de fundo (CMB) em 1965 por Arno Penzias e Robert W. Wilson, pois esta pôs fim ao debate entre o modelo de estado estacionário de Fred Hoyle e o Big Bang de Gamov, tendo em vista que este último previa um estado térmico primordial. Estes avanços, juntamente com outros experimentos observacionais paradigmáticos, como estimativas de idade do universo pelo diagrama HR da sequência principal, por exemplo, têm nos conduzido a uma visão cada vez mais detalhada e restritiva, do ponto de vista teórico, sobre o estado observado do universo.

Podemos destacar a validação, pelos surveys de altos redshifts, do chamado princípio cosmológico. O princípio cosmológico, primeiramente formulado por Einstein em 1917, tinha por objetivo simplificar a solução das equações de campo relativísticas, de modo a permitir extrair uma solução que pudesse descrever o universo. O princípio diz que o universo deve parecer o mesmo, pelo menos no que diz respeito à distribuição média 
de matéria, quando visto de qualquer ponto e qualquer direção num mesmo instante do tempo cósmico. O universo ser descrito, até onde se pode observar, por uma solução tão altamente simétrica, num conjunto de infinitas soluções possíveis das equações de Einstein, é realmente uma característica improvável de se explicar pelo acaso. Num modelo de universo que satisfaça essas condições, existem apenas duas liberdades na especificação do mesmo: a curvatura da superfície de tempo constante e a evolução temporal do fator de escala. Estes modelos são coletivamente chamados de Friedman-Robertson-Walker (FRW). Outro fato relevante é que muitos testes independentes conduzem à conclusão de que o universo é plano, ou aproximadamente plano. Essa é outra característica intrigante do universo, visto que as equações de Einstein preveem que um universo plano é um ponto crítico instável da evolução temporal.

Algo que ressalta imediatamente aos olhos é que a distribuição de matéria do universo não é completamente uniforme. A simples inspeção do ambiente à nossa volta sugere isso. Somos então levados a considerar um universo com perturbações de densidade. Essa densidade deve refletir os vínculos impostos pela causalidade. O horizonte de causalidade, ou horizonte de partícula, impõe restrições sobre a correlação entre valores de quaisquer observáveis em diferentes pontos do espaço-tempo. Nos modelos FRW satisfazendo as chamadas condições de energia forte, $\rho+3 p \geq 0$, este é da ordem de grandeza do horizonte de Hubble, $H^{-1}$, e espera-se que um valor particular de uma observável qualquer num dado ponto A, digamos a densidade, não implique em uma maior probabilidade da obtenção de algum outro valor particular para outra observável qualquer num outro ponto B se a separação entre eles for maior do que o tamanho do horizonte de Hubble. Tal observação não teria explicação num universo FRW onde se supõe que o efeito da gravidade seja sempre atrativo, pois este exibe um peculiar comportamento do horizonte: o mesmo sempre cresce mais rápido do que a separação entre observadores que acompanham a expansão do universo (separação comóvel). Isto implica que num tempo suficientemente remoto, o horizonte é sempre menor que a separação entre quaisquer dois observadores. Mais ainda, que o momento da igualdade entre estas escalas, o cruzamento do horizonte, ocorre apenas uma vez. Por tanto, encontrar uma correlação entre observáveis em pontos mais distantes que o horizonte num dado instante estaria para sempre afastado de qualquer explicação.

O mapeamento do padrão de flutuações de temperatura da CMB pelo satélite COBE 
(1989-1992), outro evento paradigmático, demonstrou exatamente esse fato. Sabemos, pelo efeito Sachs-Wolf, que as flutuações de temperatura traçam o padrão de flutuações de densidade que deram origem à estrutura em larga escala do universo. Esta observação nos mostrou o desconcertante ajuste da temperatura, a uma proporção de $10^{-5}$, entre pontos que deveriam ser causalmente desconectados, juntamente com flutuações de densidade que apresentam um peculiar padrão de correlações.

Colocado na maneira como foi o problema fundamental da cosmologia, e diante dos fatos observacionais acima mencionados, a inflação é o paradigma que melhor cumpre a proposta. Isto porque prevê que se abandonarmos a hipótese de gravidade sempre atrativa, de forma tal que o universo tenha uma fase primordial de expansão acelerada, então uma grande variedade de condições iniciais conduziria ao mesmo conjunto de propriedades fundamentais observadas do universo.

Embora, como paradigma, a inflação desfrute do mesmo status que o próprio Big Bang, este é um paradigma em busca de uma realização para além de qualquer problema conceitual. Desde sua concepção, o mecanismo de inflação passou por várias revisões. Se a inflação realmente ocorreu, não temos hoje uma evidência irrefutável de como. Os ingredientes fundamentais envolvidos na realização usual da inflação já estavam presentes em trabalhos dos anos 70, tanto de Andrei Linde, sobre a possibilidade da energia potencial de campos escalares imitar o efeito da constante cosmológica, como de Starobinsky (19791980) em conexão com efeitos de gravitação quântica. Andrei Linde e Gennady Chibisov (1978) buscavam a origem da entropia do universo num modelo com uma fase de expansão exponencial primordial, mas que conduzia a um universo altamente inomogêneo, provavelmente o primeiro exemplo do chamado problema da saída graciosa. O modelo de Alexei Starobinsky era um mecanismo de expansão exponencial sem problema de saída graciosa, mas que já partia de um universo homogêneo e isotrópico. Mas foi em 1981 que Alan Guth propôs um modelo muito mais simples que conectou o mecanismo de inflação com toda uma nova classe de problemas conceituais do Big Bang. Problemas estes antes vistos como um conjunto qualquer de condições iniciais do universo, mais do que se poderia esperar que uma teoria física fornecesse. Pode-se dizer que a inflação introduziu o elemento adicional que destacou a formulação da cosmologia perante a física como um todo.

O modelo de Guth, agora chamado de velha inflação, tinha três ingredientes abandona- 
dos em realizações posteriores da inflação: a hipótese de equilíbrio termodinâmico; a ideia de que o inflaton, campo escalar que dirige a inflação, é o mesmo campo responsável pela quebra espontânea de simetrias de calibre nas teorias de grande unificação; e que a parte efetiva da inflação acontece no falso vácuo do inflaton (mínimo local do potencial).

Neste modelo, em uma interpretação semiclássica, a inflação ocorre enquanto o campo escalar está aprisionado, por uma barreira de potencial, no mínimo local deste. A inflação termina quando efeitos de tunelamento permitem que o campo atinja o seu vácuo verdadeiro. Contudo, trata-se de transição de fase de primeira ordem (i.e. descontínua), no qual se formam regiões da nova fase, as chamadas bolhas (onde a barreira foi penetrada), que coexistem com regiões da fase anterior. A probabilidade de penetração da barreira determinava a taxa de formação das bolhas. Se a taxa de formação é pequena, a inflação dura muito, a bolhas jamais se encontram e o interior delas representa um universo isolado com densidade tendendo a zero. Se a probabilidade é alta, as bolhas se formam próximas e sua colisão produz um universo altamente inomogêneo. Isso levou ao abandono da hipótese de falso vácuo. Este é outro exemplo do problema de saída graciosa.

A alternativa veio na chamada nova inflação, no qual a o inflaton pode iniciar a inflação tanto no falso vácuo como em qualquer outro estado instável. A diferença crucial é que a parte útil da inflação não ocorre no falso vácuo, mas sim durante a lenta descida do potencial em direção ao mínimo. Isso necessita de um potencial muito plano próximo ao mínimo, satisfazendo as chamadas condições de rolamento lento, que por sua vez implicam em constantes de acoplamento muito pequenas. A constante de acoplamento regula a força das interações, que por sua vez levam ao equilíbrio. Uma constante de acoplamento pequena torna improvável o equilíbrio térmico, base da velha e nova inflação. A teoria de transições de fase cosmológica é, portanto, inaplicável, a não ser que o universo já tivesse uma grande quantidade de partículas (entropia) já de princípio, de modo a aumentar a taxa de interação, favorecendo o estabelecimento do equilíbrio.

A hipótese de equilíbrio térmico ainda assim parecia a mais natural no momento da concepção da inflação, devido própria existência da CMB implicar em uma fase térmica primordial, além do sucesso do Big Bang em prever, sob a hipótese de equilíbrio, a abundância observada de elementos primordiais. Esta foi abandonada com a paradigmática inflação caótica, que assumia uma inflação ocorrendo fora do equilíbrio com condições iniciais 
aleatórias. Um campo escalar muito deslocado do mínimo de seu potencial, possivelmente por grandes flutuações quânticas de uma fase Trans-Planckiana anterior. Supõe-se, contudo, que a inflação termine em uma fase onde a densidade de energia é dominada pelo campo escalar e este decai lentamente na matéria hodierna, de modo tal que possibilite um restabelecimento do equilíbrio. Este período é chamado reaquecimento.

A hipótese de inflação imersa nas teorias de grande unificação (que preveem que a eletrodinâmica, força nuclear fraca e forte surgem pela quebra espontânea das simetrias de uma única teoria de calibre) foi motivada por sua própria classe de problemas internos, pois previam a altas temperaturas a criação de partículas estáveis e pesadas que rapidamente se tornariam a principal componente do universo, mas que no entanto, jamais foram observadas, a mais notável delas sendo o monopólo magnético. Essa tentativa de conciliação, contudo, também não foi concretizada. O motivo para isso é a inadequação da forma do potencial do campo associado que, sendo muito íngreme, não levaria a uma duração adequada da inflação para, por exemplo, permitir que o horizonte de partícula compreendesse a região observada com temperatura homogênea da CMB, permitindo assim uma origem causal para a correlação.

Em 1999, Andreas Albrecht e João Magueijo propuseram uma mudança paradigmática na inflação. Segundo eles, se a velocidade da luz, codificada em combinações adimensionais de variáveis, for maior no universo primordial, então temos uma solução alternativa para o problema do horizonte: O horizonte no passado é maior do que o esperado se a velocidade da luz for maior. Estes autores assumiram ainda uma peculiar maneira de modificar a relatividade geral, que quebra a covariância geral das equações de Einstein, sob a hipótese de velocidade da luz variável (VSL). Sob essa hipótese, vários outros problemas previamente resolvidos pela inflação são resolvidos pela VSL.

Em um trabalho posterior, Stephon Alexander e João Magueijo (2001) conectaram uma versão alternativa de seu modelo de VSL com as então intensamente estudadas teorias de campo não comutativas. A motivação para isso foi um resultado na teoria de cordas conhecido como limite Seiberg-Witten. Este dizia que num certo limite, a teoria de cordas previa uma modificação efetiva na teoria de campos: esta seria uma teoria no espaço não comutativo, ou seja, um espaço sob a hipótese de um princípio de incerteza entre as coordenadas do espaço-tempo $\left[x^{\mu}, x^{\nu}\right] \neq 0$, abrindo a possibilidade de tratar consequências 
fenomenológicas de baixa energia da teoria de cordas. Ao longo de uma década, essas teorias tem então sido intensamente estudadas. Como definir tais teorias, tem sido uma das principais questões, mas diversas realizações demonstram algo em comum: violação da relatividade especial. Esta é a característica fundamental conectada com velocidade da luz variável. Esta ideia ofereceu o vislumbre da até então elusiva conexão entre a teoria de cordas e a inflação. Isso levou Stephon Alexander, Robert Brandenberger e João Magueijo a proporem a inflação não comutativa (2003, 2005 e 2007). Este modelo se diferencia da VSL por, diferentemente desta, ser um mecanismo de inflação, e por não se assumir violações da covariância geral das equações de campo de Einstein. Um ponto importante é que, na indefinição de como construir a teoria quântica no espaço-tempo curvo não comutativo, que remota a recentes desenvolvimentos da matemática no ramo conhecido como geometria não comutativa, recorreu-se a uma hipótese assumidamente heurística de que seria possível calcular a função de partição canônica da radiação, possibilitando uma análise da sua termodinâmica no universo primordial, baseando-se apenas no seu aspecto fenomenológico não relativístico codificado em uma deformação da relação energia-momento. Recuperouse neste modelo de inflação a então abandonada hipótese de inflação em equilíbrio térmico e com uma nova mudança de paradigma: Inflação poderia ser dirigida pela radiação em altas temperaturas, não por algum campo escalar hipotético.

Essa hipótese de trabalho, isto é, codificar aspectos de uma física de altas energias não completamente entendida na modificação da relação energia-momento, não é nova na literatura. Já tinha sido empregada antes para estudar a robustez do cálculo da radiação Hawking com respeito a modificações de uma física de altas energias e na própria inflação com o intuito de testar a invariância de suas previsões com respeito a efeitos desconhecidos da física de altas energias. No mais, vários trabalhos mostraram que em algumas circunstâncias, várias tentativas de modificar a formulação da teoria de campos, por modificações dos esquemas de quantização, ou princípio de incerteza energia-momento modificados, por exemplo, terminavam por se manifestar através de violações na simetria relativística da teoria.

A presente tese estuda, de maneira não exaustiva, a hipótese Trans-Planckiana na inflação, isto é, como o mecanismo de inflação pode ser afetado por violações da relatividade especial oriundos de uma física de altas energias. Mais ainda, estuda o significado 
conceitual e teórico da relação de dispersão no contexto quântico da representação de simetrias e definição da teoria quântica da matéria. Ideia essa em conexão com os próprios fundamentos da teoria quântica expressos em resultados fundamentais devido a Von Neumann, Wigner, Stone, Wightman e Haag. Explora a conexão entre esta ideia e a ideia de espaço-tempo não comutativo, regras de quantização deformadas e princípio de incerteza modificado, algumas das principais motivações teóricas para uma violação da relatividade especial em altas energias. Dentre esses, destaca a ideia de não comutatividade, cuja estrutura está intimamente ligada ao problema de representação de simetrias da teoria quântica e motiva o modelo de inflação não comutativa, principal modelo explorado nessa tese. No tocante a essa conexão, estuda como o problema de representação de simetrias se generaliza para simetrias de espaços não comutativos através de estruturas mais gerais que grupos, as álgebras de Hopf, revalidando o modelo de inflação não comutativa.

Apesar de os resultados originais dessa tese estarem essencialmente nos capítulos 7 e 8. dispersos ao longo de todos os capítulos, existem interpretações, observações e considerações originais desse autor sobre as teorias fundamentais sobre as quais se apoia essa tese. Fruto da maneira original e particular como cada pesquisador deve olhar as bases da sua ciência. Observamos ainda que a estrutura em que os nossos resultados originais são apresentados, apesar de baseada no primeiro trabalho publicado desta tese, é diferente deste, devido a novos resultados que estão por ser submetidos, mas já estão na tese. 


\section{Capítulo 2}

\section{A estrutura clássica do espaço-tempo e matéria}

\subsection{Introdução}

Falar do universo é, em princípio, falar da distribuição de matéria, e toda sua informação associada, ao longo de grandes distâncias e, por conseguinte, dos fatores que moldam tal distribuição. Na visão Newtoniana da física, apenas forças podem afetar a distribuição da matéria. Em última análise, o problema cosmológico envolve todas as forças a que a matéria está submetida, mas são mais relevantes as forças que criam as correlações mais fortes na distribuição da matéria no limite de longas distâncias. Sobressaem-se, então, as forças que mais lentamente decaem em função da distância, como a força gravitacional e a eletromagnética. Mais ainda, são mais relevantes as forças que decaem mais lentamente quando consideramos o efeito líquido associado à distribuição típica de suas fontes no universo. A gravidade então se sobressai sobre a força eletromagnética como a força mais relevante no universo, por afetar todos os corpos da mesma maneira. Conforme dito, essa é uma descrição Newtoniana do universo. Numa descrição quântica, efeitos de flutuações podem afetar a distribuição de quaisquer observáveis através do espaço-tempo.

Começaremos aqui uma compilação de conceitos e resultados relevantes na descrição clássica da matéria e do universo, partindo do conceito sobre o qual repousa a ideia de força: o referencial inercial, através do qual chegaremos ao conceito de referencial localmente inercial e, por consequência lógica, ao conceito de espaço-tempo curvo, sobre o qual se ergue toda a cosmologia. Desde o início, construiremos a notação útil para tratar a geometria de um espaço-tempo curvo, com ênfase na descrição geométrica do grupo de Poincaré, foco desta tese, deixando abordagens mais algébricas para capítulos posteriores. As principais referências para esse capítulo são: de Felice e Clarke (1990), Landau e Lifshitz (1980), 
Hawking e Ellis (1973), Wald (1984), Stewart (1993), Weinberg 1972), do Carmo (1992b), Nakahara (2003), Heller (1992), do Carmo (2005), Schrödinger (1950), Spivak (1971) e Friedlander e Joshi (1998).

\subsection{O espaço-tempo plano}

As leis de Newton postulam a existência de um sistema de coordenadas de tempo e espaço $(t, x)$ chamado referencial inercial, no qual as trajetórias $x_{i}(t)$ dos corpos obedecem equações diferenciais de segunda ordem no tempo e lineares na derivada de maior ordem:

$$
\begin{aligned}
m_{1} \frac{d^{2} x_{1}}{d t^{2}} & =F_{1}\left(\frac{d x_{1}}{d t}, \ldots, \frac{d x_{N}}{d t}, x_{i}, \ldots, x_{j}, t\right) \\
& \vdots \\
m_{N} \frac{d^{2} x_{N}}{d t^{2}} & =F_{N}\left(\frac{d x_{1}}{d t}, \ldots, \frac{d x_{N}}{d t}, x_{i}, \ldots, x_{j}, t\right)
\end{aligned}
$$

No contexto geral das equações diferenciais, isso é uma severa restrição à generalidade. Tal restrição, que não afetaria a consistência lógica da teoria caso não fosse assumida, é o que se pode entender por lei física. Neste sistema de coordenadas particular, a menos de uma constante intrínseca ao corpo que se move e que multiplica a derivada segunda- a massa $m_{i}$, que mede a resistência ao movimento- o que aparece igualado à derivada segunda da posição é o que se define por força. A possibilidade, por postulado, de unicamente determinar as derivadas de segunda ordem nas equações de movimento é o que torna o conceito de força unicamente definido. As leis de Newton postulam então um tipo de equação diferencial muito particular no qual a existência e unicidade de soluções para um dado conjunto de condições iniciais podem ser estabelecidas, levando a um modelo de teoria física baseado no problema do valor inicial, ou problema de Cauchy, que influenciou toda a formulação da física posterior.

Ao postular um particular sistema de coordenadas, determinado pela forma das leis físicas neste, todos aqueles obtidos deste por transformações que não afetam a forma das equações em questão definem toda uma classe de equivalência de referenciais. Somos então levados a considerar as transformações entre referenciais inerciais, o que define a relação de equivalência entre os mesmos: as transformações de Galileu, que formando um conjunto fechado à composição e inversa, definem um grupo, cada uma destas transformações 
é unicamente definida pela especificação de 10 parâmetros:

$$
\begin{aligned}
& \vec{x} \rightarrow R \overrightarrow{x^{\prime}}+\vec{v} t+\vec{d} \\
& t \rightarrow t^{\prime}+\tau
\end{aligned}
$$

A eletrodinâmica, contudo, não é invariante por transformações de Galileu. A relatividade especial postula então uma modificação dessa classe de transformações de modo a preservar a formulação da eletrodinâmica nos diferentes referenciais inerciais: as transformações de Lorentz. Estas são caracterizadas por serem transformações afins- isto é, transformações lineares seguidas por translações- e por preservarem a estrutura de cone de luz embutida nas equações de Maxwell.

Preservar a estrutura de cone de luz equivale ao postulado de Einstein de que a velocidade da luz é a mesma para todos os observadores inerciais. Este postulado é expresso matematicamente pelo requerimento:

$$
d s=0 \Leftrightarrow d s^{\prime}=0
$$

onde

$$
d s^{2}=d t^{2}-d x^{2}-d y^{2}-d z^{2}
$$

e $d s^{\prime}$ é a transformação de $d s$ no novo sistema de coordenadas.

A equação 2.4 define a métrica de Minkowski. Uma métrica é uma forma bilinear simétrica não degenerada 1 que define um produto interno no espaço tangente de cada ponto $p$. O espaço tangente em $p$, denotado por $T_{p} \mathcal{M}$, é constituído de todas os vetores tangentes a todas as curvas que passam em $p$. Num dado sistema de coordenadas, este é identificado com o espaço vetorial gerado pelas derivadas parciais $\partial_{\mu}$ em $p$, denotadas $\partial_{\mu}$. O vetor tangente de uma dada curva $\gamma:(a, b) \rightarrow \mathbb{R}^{4}$, no ponto $\gamma\left(t^{\prime}\right)$ é denotado por $\frac{p}{d \gamma\left(t^{\prime}\right)} d$, faz corresponder uma derivada direcional em $\mathcal{C}$, o conjunto das funções diferenciáveis em $\mathcal{M}$, pela regra $\left.\frac{d}{d t} f(\gamma(t))\right|_{t=t^{\prime}}, f \in \mathcal{C}$, com a qual é identificado. Esta última definição é independente do sistema de coordenadas usado.

Sendo os elementos de $T_{p} \mathcal{M}$ identificados com os funcionais lineares com suporte em $p$ satisfazendo a regra de Leibniz, isto é $\underset{p}{X}(f g)=\underset{p}{\underset{p}{X} f) g}+\underset{p}{f} \underset{\mathrm{X}}{\mathrm{X}} \mathrm{g})$, que atuam no espaço $\mathcal{C}$

\footnotetext{
${ }^{1}$ Uma forma bilinear num espaço vetorial real $V$ é uma regra ()$: V \times V \rightarrow \mathbb{R}$ tal que $(u, \lambda v+\beta v)=$ $\lambda(u, v)+\beta(u, v)$ e $(\lambda u+\beta u, v)=\lambda(u, v)+\beta(u, v)$. É dita não degenerada se $(u, v)=0$ para todo $u$ se, e somente se, $v=0$. É dita simétrica se $(u, v)=(v, u)$
} 
de funções diferenciáveis no $\mathbb{R}^{4}$, o que simbolicamente denotamos $\underset{p}{X} \in T_{p} \mathcal{M}: \phi \in \mathcal{C} \rightarrow \mathbb{R}$, definimos um campo vetorial $X$ como um operador linear satisfazendo a regra de Leibniz tal que $X: \mathcal{C} \rightarrow \mathcal{C}$. Denotaremos o espaço vetorial dos campos vetoriais por $\mathcal{X}(\mathcal{M})$.

Uma aplicação diferenciável $\psi: \mathcal{U} \rightarrow \mathcal{V}$, sendo $\mathcal{U}$ e $\mathcal{V}$ abertos do $\mathbb{R}^{n}$, induz uma aplicação linear entre espaços tangentes. A diferencial $d \psi_{p}$, no ponto $p$, de uma transformação diferenciável $\psi: \mathcal{U} \rightarrow \mathcal{V}$ é um mapa linear entre os espaços tangentes $T_{p} \mathcal{U}$ e $T_{\psi(p)} \mathcal{V}$, denotado por $d \psi_{p}: T_{p} \mathcal{U} \rightarrow T_{\psi(p)} \mathcal{V}$, tal que, qualquer que seja a curva $\gamma:(a, b) \rightarrow \mathcal{U}$ satisfazendo $\gamma\left(t^{\prime}\right)=p$, é válido:

$$
\left.\frac{d}{d t} \psi(\gamma(t))\right|_{t=t^{\prime}}=d \psi_{p}\left(\frac{d \gamma\left(t^{\prime}\right)}{d t}\right)
$$

A maneira mais geral de escrever uma métrica $<,>: \mathcal{X}(\mathcal{M}) \times \mathcal{X}(\mathcal{M}) \rightarrow \mathbb{R}$ é dada por $d s^{2}=g_{\mu \nu} d x^{\mu} d x^{\nu}$, onde a forma diferencial $d x^{\mu}$ é definida pela escolha em cada ponto do funcional linear em $T_{p} \mathcal{M}$, denotado $\underset{p}{d x^{\mu}}$, satisfazendo $\underset{p}{d \underset{p}{\mu}} \underset{p}{\left(\partial_{\nu}\right)}=\delta_{\nu}^{\mu}$, sendo o produto de diferenciais, denotado por $d x^{\mu} \otimes d x^{\nu}$ ou $d x^{\mu} d x^{\nu}$, definido tal que $d x^{\mu} \otimes d x^{\nu}(u, v)=$ $d x^{\mu}(u) \cdot d x^{\nu}(v)$, para $u, v \in \mathcal{X}(\mathcal{M})$. Os funcionais lineares no espaço tangente formam o chamado espaço dual, denotado por $T_{p} \mathcal{M}^{*}$, sendo o produto de diferenciais $d x^{\mu} \otimes d x^{\nu}$ uma base no espaço dos funcionais bilineares no espaço tangente $\otimes^{2} T_{p} \mathcal{M}^{*}$. O elemento de linha de Minkowski é então especificado por $g_{\mu \nu}=\eta_{\mu \nu}$, onde:

$$
\eta_{\alpha \beta}=\left(\begin{array}{cccc}
1 & 0 & 0 & 0 \\
0 & -1 & 0 & 0 \\
0 & 0 & -1 & 0 \\
0 & 0 & 0 & -1
\end{array}\right)
$$

$d s$ define uma medida no espaço de trajetórias no espaço-tempo, onde a medida de cada curva $\gamma(t):(a, b) \rightarrow \mathbb{R}^{4}$ é dada por

$$
\int d s=\int d t \sqrt{\left\langle\frac{d \gamma}{d t}, \frac{d \gamma}{d t}\right\rangle_{\gamma(t)}}=\int d t \sqrt{\eta_{\mu \nu} \frac{d \gamma^{\beta}}{d t} \frac{d \gamma^{\beta}}{d t}}
$$

A condição 2.3 então implica que:

$$
d s=a(x) d s^{\prime}, \quad a(x) \neq 0 \text { para todo } x
$$

O que se justifica por um teorema da teoria da medida e integração conhecido como teorema de Radon-Nikodym Isnard (2007). Este teorema exercerá um papel importante em conexão com representação de operadores em espaços de Hilbert mais adiante. 
O requerimento de linearidade/afinidade decorre da imposição de que partículas livres, que se movem em linha reta e a velocidade constante, são mapeadas em partículas livres, movendo-se em linhas retas e a velocidade constante no novo referencial-Princípio da Inércia de Galileu - Este requerimento implica $a(x)$ constante, uma vez que $d s^{2}$ muda com a diferencial da transformação de coordenadas:

$$
d s^{\prime 2}=g_{\alpha \beta} d x^{\prime \alpha} d x^{\prime \beta}=\eta_{\alpha \beta} \frac{\partial x^{\prime \alpha}}{\partial x^{\mu}} \frac{\partial x^{\prime \beta}}{\partial x^{\nu}} d x^{\mu} d x^{\nu}
$$

Uma vez que $d s^{\prime}=c d s$, uma simples redefinição das unidades de medida no referencial transformado faz $d s^{\prime}=d s$. Sem o requerimento $a(x)$ constante, estaremos considerando um grupo de Lie de 15 parâmetros conhecido como grupo conforme.

Reciprocamente, a propriedade de deixar invariante a elemento de linha de Minkowski determina unicamente as transformações de Lorentz, isto é, não existe transformação inversível e diferenciável de inversa diferenciável, um difeomorfismo, que tenha essa propriedade e que não pertença ao grupo de Lorentz. Por tanto, escrevemos os seus elementos como:

$$
x^{\prime \mu}=\Lambda_{\nu}^{\mu} x^{\nu}+b
$$

Sendo assim, $d s^{2}$ define uma geometria Lorentz invariante no espaço-tempo. Isto é, uma forma Lorentz invariante de definir medidas em curvas, superfícies, volumes e análogos em todas as dimensões. A menos de uma constante, absorvível por uma mudança das unidades de medida de espaço, é a única geometria compatível com os postulados da relatividade especial.

A métrica nos permite classificar as curvas que conectam dois pontos quaisquer do espaço-tempo, no que diz respeito ao estabelecimento da noção de causalidade relativística. Um axioma fundamental da física é que causa precede efeito. Esta noção é certamente afetada pela relatividade da simultaneidade proposta na relatividade especial. Pontos separados por uma distância tipo espaço são, em algum outro referencial inercial, simultâneos. Desse modo, uma versão Lorentz invariante de causalidade implica que um dado ponto $q$ só pode afetar o seu cone de luz do futuro $J^{+}(x)$, isto é, a região dos pontos que podem ser conectados por uma curva que parte de $q$ e na qual o vetor tangente em cada ponto é nulo $\left(\frac{d \gamma}{d t}, \frac{d \gamma}{d t}\right)=0$, ou tipo-tempo $\left(\frac{d \gamma}{d t}, \frac{d \gamma}{d t}\right)>0$ e que aponta para o futuro $\left(\frac{d \gamma}{d t}, \partial_{t}\right)>0$. Reciprocamente, definimos o cone de luz do passado $J^{-}(x)$, como 
o conjunto de todos os eventos que podem afetar $x$. Dizemos então que $q$ é causalmente desconectado de $p$ se $p \notin J^{+}(q) \cup J^{-}(q)$, o que equivale a $q \notin J^{+}(p) \cup J^{-}(p)$, estabelecendo uma relação de equivalência que substitui a noção pré-relativística de simultaneidade absoluta. Esta condição pode ainda ser enunciada de outra maneira: O vetor tangente em algum ponto de qualquer curva que liga $q$ a $p$ é necessariamente tipo espaço $\left(\frac{d \gamma}{d t}, \frac{d \gamma}{d t}\right)<0$.

A métrica também define um conjunto privilegiado de curvas, as geodésicas, curvas cujos comprimentos são estacionários com respeito a deformações das mesmas mantendo-se fixos os pontos extremos ${ }^{2}$ :

$$
\frac{\delta}{\delta \gamma^{\mu}(t)} \int_{\gamma} d s=0
$$

No caso de uma geometria de métrica positiva definida, i.e. $\left\langle v, v>_{p} \geq 0\right.$, a geodésica é a curva de menor comprimento entre dois pontos suficientemente próximos, isto é, existe uma vizinhança $\mathcal{V}$ tal que existe uma única geodésica que liga $p, q \in \mathcal{V}$ e esta é a curva de menor comprimento que liga $p$ a $q$. Mais ainda, sendo $\gamma:(a, b) \rightarrow \mathbb{R}^{4}$ a curva de menor comprimento entre dois pontos, esta é uma das geodésicas que os ligam. No caso do espaço-tempo de topologia trivial $\left(\mathbb{R}^{4}\right)$ e métrica de Minkowski, as geodésicas representam as trajetórias das partículas livres, e maximizam o comprimento da curva. As geodésicas podem ser reparametrizadas, como qualquer outra curva regular - isto é, que admite reparametrização local tal que o vetor tangente seja definido e não se anula em nenhum ponto-, de modo que $\left(\frac{d \gamma}{d t}, \frac{d \gamma}{d t}\right)=$ const.

Sob essas mesmas condições, o ponto $q$ de coordenadas $\left(x^{0}, x^{1}, x^{2}, x^{3}\right)$ e $q^{\prime}$ de coordenadas $\left(x^{\prime 0}, x^{\prime 1}, x^{\prime 2}, x^{\prime 3}\right)$ causalmente conectados são aqueles entre os quais existe uma geodésica que os conecta e é tipo tempo, ou nula. O que é equivalente à:

$$
\left(x^{0}-x^{\prime 0}\right)^{2}-\left(x^{1}-x^{\prime 1}\right)^{2}-\left(x^{2}-x^{\prime 2}\right)^{2}-\left(x^{3}-x^{\prime 3}\right)^{2} \geq 0
$$

\footnotetext{
${ }^{2}$ Seja $\mathcal{F}: \mathcal{C}\left(\mathbb{R}^{n}\right) \rightarrow \mathbb{R}$ um funcional, a derivada funcional é definida como o funcional linear contínuo $\frac{\delta \mathcal{F}}{\delta \gamma}$, tal que $\lim _{\epsilon \rightarrow 0} \frac{\mathcal{F}\left(\gamma^{\prime}(x)+\epsilon f(x)\right)-\mathcal{F}\left(\gamma^{\prime}(x)\right)}{\epsilon}=\int \frac{\delta \mathcal{F}}{\delta \gamma} f(x) d x$ para todo $f(x) \in \mathcal{C}_{c}^{\infty}\left(\mathbb{R}^{4}\right)$ da funções infinitamente diferenciáveis de suporte compacto. A ação de $\frac{\delta \mathcal{F}}{\delta \gamma}$ em $f(x),<\frac{\delta \mathcal{F}}{\delta \gamma}, f(x)>$, é denotada por $\int \frac{\delta \mathcal{F}}{\delta \gamma} f(x) d x$. Continuidade significa que $<\frac{\delta \mathcal{F}}{\delta \gamma}, f_{n}(x)>\rightarrow 0$ se todos os $f_{n}(x)$ se anulam fora de algum compacto $K$ e $\sup \left|\frac{\partial^{N}}{\partial x^{i}{ }_{1} \ldots \partial x^{i} N} f_{n}(x)\right| \rightarrow 0$, para todo $N$ quando $n \rightarrow \infty$.
} 


\subsection{O espaço-tempo curvo}

\subsubsection{A localidade na descrição da física}

A ideia de localidade como requerimento na formulação da física remete a Faraday. De certo modo, diz que a topologia do espaço-tempo é um vínculo imposto à formulação de qualquer lei física. Localidade é o requerimento de que não pode, a princípio, haver lei física relacionando observáveis em $p$ e cuja formulação/verificação não possa ser feita apenas com o conhecimento da informação numa vizinhança arbitrariamente pequena de $p$.

O mecanismo através do qual a noção de localidade é implementada na física é o conceito de campo. Se um observador determina que a informação no ponto $A$ do espaçotempo influencia a informação no ponto $B$, ele deverá, a princípio, poder constatar que $A$ influenciou a informação em cada um dos pontos de um caminho que liga $A$ a $B$. Desse modo, é necessário especificar alguma informação em cada ponto do espaço-tempo, sendo esta especificada por um conjunto de números reais (resultado de um conjunto de medições) que definem um conjunto de funções $\Phi_{i}(x), i=1, \ldots N$. Mais ainda, dito de uma maneira matemática, a localidade diz que as leis físicas relacionando o valor dos campos em $p$ são formuladas através do $\boldsymbol{g e r m e}$ dos campos em $p$, isto é, a classe de equivalência de funções idênticas em alguma vizinhança de $p$. Podemos ainda expressar de outra maneira a ideia de localidade: A leis físicas envolvendo as informações em $p$ são funcionais não identicamente nulos dos campos $\mathcal{F}: \Phi \rightarrow \mathbb{R}$, com suporte em $p$, igualados a zero:

$$
\mathcal{F}[\Phi(x)]=0
$$

Podemos assumir que os campos são infinitamente diferenciáveis, uma vez que o grau de diferenciabilidade do campo possivelmente não é uma observável física. Podemos assumir que os campos tem o suporte compacto, uma vez que as condições no infinito assintótico não deveriam afetar a formulação de uma física local. Nesse caso, podemos definir uma noção de proximidade entre os campos: duas configurações de um dado campo $\phi_{1}(x)$ e $\phi_{2}(x)$ são próximas quando sua diferença é arbitrariamente pequena em algum sentido. Um campo é arbitrariamente próximo a outro quando o sup $\left|\partial_{i}\left(\phi_{1}(x)-\phi_{2}(x)\right)\right|<\epsilon$. Nesse caso, podemos assumir o que funcional 2.13 é contínuo $\lim _{N} \mathcal{F}\left[\Phi_{N}(x)\right]=0$, quando uma sequência de campos $\Phi_{N}$ aproxima um campo fisicamente realizável. Pelo mesmo argumento que 
assumimos os campos infinitamente diferenciáveis, podemos assumir um funcional diferenciável, expandindo-se o funcional em termos de funcionais multilineares:

$$
\mathcal{F}\left(\Phi^{\prime}(x)+\epsilon \Psi(x)\right)=C+<\frac{\delta \mathcal{F}}{\delta \Phi(x)}, \Psi(x)>\epsilon+<\frac{\delta^{2} \mathcal{F}}{\delta \Phi(x) \delta \Phi(y)}, \Psi(x) \Psi(y)>\epsilon^{2}+\mathcal{O}\left(\epsilon^{3}\right)
$$

e observando que o funcional linear contínuo mais geral com suporte em $p$ é dado por:

$$
\sum_{N=0}^{M} \frac{\partial^{N}}{\partial x^{i_{1}} \ldots \partial x^{i_{N}}} \delta(x-p)
$$

concluímos que $\mathcal{F}[\Phi(x)]$ é uma função de $\Phi(x)$ e suas derivadas parciais em $p$, o que implica que, sob as hipóteses acima, equações diferenciais parciais envolvendo derivadas dos campos no mesmo ponto são a única formulação local possível das leis físicas. O requerimento de invariância translacional das leis implica que:

$$
\mathcal{F}(\Phi(x+a))=0, \quad \text { para todo } a
$$

Observe ainda que a expansão 2.14 embute o resultado de que o funcional multilinear contínuo mais geral é da forma:

$$
B(\Psi(x), \Phi(y))=<\mathcal{B}, \Psi(x) \cdot \Phi(y)>
$$

onde $\mathcal{B}$ é um funcional linear contínuo- teorema de Swartz Friedlander e Joshi (1998).

A gravidade Newtoniana incorpora localidade por se formular através de campos, mas é incompatível com a causalidade relativística - uma mudança na distribuição de matéria numa região instantaneamente se faz sentir em todo o universo. Mais ainda, a gravidade newtoniana é outra teoria invariante por transformações de Galileu, não de Lorentz. A equivalência entre massa inercial e gravitacional, a afirmação da universalidade da ação da gravidade, já conhecida e demonstrada na física desde Galileu, foi um dos ingredientes na formulação da gravidade Newtoniana. A terceira lei, da ação e reação, juntamente com o princípio da equivalência, implica que a força gravitacional sentida por um corpo é proporcional à sua massa e à massa da fonte da força sobre ele. A equivalência entre massa gravitacional e massa inercial implica que, na vizinhança de cada ponto de um campo gravitacional arbitrário, existe uma transformação de coordenadas não Galileana que anula, na vizinhança desse ponto, o efeito da gravidade na mecânica, recuperando a forma da lei de movimento na ausência de gravidade: 


$$
m_{i} \frac{d^{2} x}{d t^{2}}=m_{i} g+\sum F\left(x_{i}-x_{j}\right)
$$

submetida à mudança de coordenadas:

$$
\begin{aligned}
x_{i}^{\prime} & =x_{i}-\frac{1}{2} g t^{2} \\
t^{\prime} & =t
\end{aligned}
$$

nos conduz à:

$$
m_{i} \frac{d^{2} x^{\prime}}{d t^{\prime 2}}=\sum F\left(x_{i}^{\prime}-x_{j}^{\prime}\right)
$$

O princípio da equivalência foi o princípio guiador de Einstein para a reformulação relativística da gravitação. Einstein elegeu o princípio da equivalência como a regra fundamental a definir o efeito da gravidade sobre todas as leis físicas, não apenas sobre a mecânica:

Princípio da equivalência entre gravitação e inércia: Na presença de um campo gravitacional arbitrário, existe uma transformação de coordenadas $\Psi_{x}: \mathcal{U} \rightarrow \mathcal{V}$ na vizinhança $\mathcal{U}$ de cada ponto $x$ que recupera, em $x$, a forma das leis no referencial inercial na ausência de gravidade.

Vemos aqui a emergência de um novo paradigma: Por um lado, uma classe particular de referenciais é privilegiada, os localmente inerciais, nos quais a forma das leis físicas é pré-estabelecida. Por outro lado, a diferença crucial é a inexistência, em geral, de referencial global privilegiado. Ou seja, a impossibilidade de, em geral, recobrir o espaço-tempo $\mathcal{M}$ por tais sistemas de coordenadas $\Psi_{x}: \mathcal{U}_{x} \subset \mathcal{M} \rightarrow \mathcal{V} \subset \mathbb{R}^{4}$ de tal modo que se $\mathcal{U}_{x} \cap \mathcal{U}_{y} \neq \emptyset$, então $\Psi_{x}^{-1} \circ \Psi_{y}=I d$. Os referenciais inerciais de Newton são substituídos pelos referencias inerciais locais de Einstein. A classe de transformações de coordenadas considerada, que era restrita tanto na física Newtoniana, como na relatividade especial, passa a incluir todos os difeomorfismos locais possíveis.

Isso nos leva naturalmente ao conceito de variedade topológica diferenciável de dimensão $N$ :

Variedade topológica diferenciável de dimensão $N$ é um conjunto $\mathcal{M}$ mais uma família de aplicações biunívocas $\Psi_{\alpha}: \mathcal{U}_{\alpha} \rightarrow \mathbb{R}^{N}$ tais que: 
- Cada $\Psi_{\alpha}\left(\mathcal{U}_{\alpha}\right)$ é um aberto de $\mathbb{R}^{N}$

- $\bigcup_{\alpha} \mathcal{U}_{\alpha}=\mathcal{M}$

- Se $\mathcal{U}_{\alpha} \cap \mathcal{U}_{\beta} \neq \emptyset$, então $\Psi_{\alpha} \circ \Psi_{\beta}^{-1}: \Psi_{\beta}\left(\mathcal{U}_{\alpha} \cap \mathcal{U}_{\beta}\right) \rightarrow \Psi_{\beta}\left(\mathcal{U}_{\alpha} \cap \mathcal{U}_{\beta}\right)$ é diferenciável $\left(\mathcal{C}^{\infty}\right)$ e inversível, isto é, um difeomorfismo.

- A família $\Psi=\left\{\left(\mathcal{U}_{\alpha}, \Psi_{\alpha}\right)\right\}$, chamada atlas de coordenadas, é maximal com respeito às condições anteriores, ou seja, qualquer outra família $\Phi=\left\{\left(\mathcal{V}_{\alpha}, \Phi_{\alpha}\right)\right\}$ cuja união com $\Psi$ ainda satisfaça as condições anteriores, é subconjunto de $\Psi$.

Dizemos então que o conjunto $\Psi$ define uma estrutura diferenciável no espaço topológico $\mathcal{M}$. A estrutura diferenciável nos permite estender noções previamente definidas no $\mathbb{R}^{n}$, como o espaço tangente em cada ponto, a um espaço mais geral. Um resultado fundamental na caracterização das variedades é o teorema de Whitney do Carmo (2005) do Carmo (1992a) que diz que toda variedade é uma superfície regular de um espaço $\mathbb{R}^{n}$ de dimensionalidade suficientemente elevada. Onde superfície regular de dimensão $k$ é um subconjunto $\mathcal{S}$ do $R^{n}$ tal que todo ponto de $\mathcal{S}$ admite uma vizinhança $\mathcal{V}$ e um difeomorfismo $\phi: \mathcal{V} \rightarrow \mathcal{U} \subset \mathbb{R}^{n}$ onde $\mathcal{U}$ é um aberto do $\mathbb{R}^{n}$ tal que $\phi(\mathcal{V} \cap \mathcal{S})=\mathcal{U} \cap\left\{x^{i}=0 ; i>k\right\}$. Uma variedade Riemanniana é uma variedade com uma métrica positiva definida em cada ponto. Uma variedade Riemanniana sempre pode ser mergulhada isometricamente no $\mathbb{R}^{n}$, isto é, identificada com uma superfície regular do $R^{n}, \mathcal{S}$, através do mapa $\psi: \mathcal{M} \rightarrow \mathcal{S}$, satisfazendo $<u, v>_{p}=<d \psi_{p}(u), d \psi_{p}(v)>_{\psi(p)}$.

Poderíamos nos perguntar qual, segundo o princípio da equivalência, é a forma mais geral de codificar a informação sobre o campo gravitacional. Para tal, consideremos a trajetória de uma partícula em queda livre. Segundo o princípio, no referencial inercial, a equação da queda livre equivale à equação da partícula livre:

$$
\frac{d^{2} x^{\mu}}{d t^{2}}=0
$$

Ou, a mesma equação escrita na forma Lorentz invariante:

$$
\frac{d^{2} x^{\mu}}{d s^{2}}=0, \quad d s^{2}=\eta_{\mu \nu} d x^{\mu} d x^{\nu}
$$

Num sistema de coordenadas arbitrário, esta equação se escreve: 


$$
\frac{d}{d s}\left(\frac{d \bar{x}^{\mu}}{d s^{2}} \frac{\partial x^{\alpha}}{\partial \bar{x}^{\mu}}\right)=0, \quad d s^{2}=\eta_{\mu \nu} \frac{\partial x^{\mu}}{\partial \bar{x}^{\alpha}} \frac{\partial x^{\nu}}{\partial \bar{x}^{\beta}} d \bar{x}^{\alpha} d \bar{x}^{\beta}
$$

Estas equações se reduzem à:

$$
\frac{d^{2} \bar{x}^{\mu}}{d s^{2}}+\Gamma_{\alpha \beta}^{\mu} \frac{d \bar{x}^{\alpha}}{d s} \frac{d \bar{x}^{\beta}}{d s}=0, \quad d s^{2}=g_{\alpha \beta} d \bar{x}^{\alpha} d \bar{x}^{\beta}
$$

onde

$$
\Gamma_{\alpha \beta}^{\mu}=\frac{\partial x^{\mu}}{\partial \bar{x}^{\tau}} \frac{\partial \bar{x}^{\tau}}{\partial x^{\alpha} \partial x^{\beta}}
$$

É possível mostrar que a relação entre $g_{\alpha \beta}$ e $\Gamma_{\alpha \beta}^{\mu}$ é dada por:

$$
\Gamma_{\alpha \beta}^{\mu}=\frac{1}{2} g^{\mu \tau}\left(\partial_{\alpha} g_{\tau \beta}+\partial_{\beta} g_{\alpha \tau}-\partial_{\tau} g_{\alpha \beta}\right)
$$

sendo $g^{\alpha \beta}$ definido pela condição $g^{\alpha \tau} g_{\tau \beta}=\delta_{\beta}^{\alpha}$.

Esta é a equação da geodésica numa métrica arbitrária $g_{\alpha \beta}$, sendo $\Gamma_{\alpha \beta}^{\mu}$ os símbolos de Christoffel. Somos levados à notável conclusão de que a gravidade é codificada numa deformação da métrica. Elegemos então a métrica ao status de campo físico, determinado pela distribuição de matéria. Um campo cuja existência e estrutura é consequência lógica do princípio da equivalência. No referencial localmente inercial em $x$, esta métrica deve então assumir a forma da métrica de Minkowski, e os símbolos de Christofell devem se anular em $x$.

A maneira mais elegante de implementar o princípio da equivalência é através do princípio da covariância geral: Um sistema de equações diferenciais parciais expressando uma lei física num conjunto de campos é válido na presença da gravidade se:

1. O sistema depende de $g_{\alpha \beta}$ e $\Gamma_{\alpha \beta}^{\mu}$ de tal modo que quando $g_{\alpha \beta}=\eta_{\alpha \beta}$ e $\Gamma_{\alpha \beta}^{\mu}=0$ as equações se reduzem à forma prescrita pela relatividade especial no referencial inercial e na ausência de gravidade.

2. As equações são geralmente covariantes, isto é, mantém a mesma forma quando expressas em sistemas de coordenadas locais arbitrários. 


\subsubsection{A formulação tensorial}

A covariância geral é reflexo da inexistência de referencial privilegiado no que diz respeito a considerações sobre leis físicas envolvendo mais que um ponto, ou seja, o requerimento de que todos os sistemas de coordenadas locais $\left(\mathcal{U}_{\alpha}, \Psi_{\alpha}\right)$ sejam equivalentes no que diz respeito à forma das leis físicas sobre a vizinhança $\mathcal{U}_{\alpha}$. Numa teoria local, que, por conseguinte, envolve o conceito de campo, isso é atingido através da noção de campo

\section{tensorial.}

Um tensor é um funcional linear no espaço produto de $N$ cópias do espaço vetorial $\mathcal{V}$ :

$$
T: \underbrace{\mathcal{V} \times \cdots \mathcal{V} \times \mathcal{V}}_{N} \rightarrow \mathbb{R}
$$

tendo o espaço-tempo $\mathcal{M}$ estrutura de variedade diferenciável, existe em cada ponto um espaço vetorial dado pelo espaço tangente $T_{p} \mathcal{M}$, bem como o espaço dual $T_{p} \mathcal{M}^{*}$, dos funcionais lineares no espaço tangente em $p$.

Os funcionais multilineares do tipo

$$
T: \underbrace{T_{p} \mathcal{M}^{*} \cdots \times T_{p} \mathcal{M}^{*}}_{r} \times \underbrace{T_{p} \mathcal{M} \cdots \times T_{p} \mathcal{M}}_{s} \rightarrow \mathbb{R}
$$

definem o espaço vetorial $\mathcal{T}_{s}^{r}(p)$ dos tensores do tipo $(r, s)$ em $p$. Segue da definição que $\mathcal{T}_{s}^{r}(p)=\otimes^{r} T_{p} \mathcal{M} \otimes^{s} T_{p} \mathcal{M}^{*}$. Dizemos que estes tensores tem $r$ indices contravariantes e $s$ indices covariantes.

Dado um sistema de coordenadas locais denotado por $x^{\mu}, \mu=1, \ldots, n$, escolhemos então uma base coordenada $\partial_{\mu}, \mu=1, \ldots, n$, das derivadas parciais em $p$, para o espaço tangente $T_{p} \mathcal{M}$ e a base dual $\underset{p}{\underset{p}{\mu}}, \mu=1, \ldots, n$, definida por $\left.\underset{p}{\left(d x_{p}^{\mu}\right.}, \underset{p}{\partial_{\nu}}\right)=\delta^{\mu}{ }_{\nu}$, para $T_{p} \mathcal{M}^{*}$. Aqui, $(\eta, X)$ denota a ação do funcional $\eta \in T_{p} \mathcal{M}^{*}$ no vetor $X \in T_{p} \mathcal{M}$. Definimos então uma base em $\mathcal{T}_{s}^{r}(p)$ por:

$$
\underset{p}{\partial_{\mu_{1}}} \otimes \underset{p}{\partial_{\mu_{2}}} \cdots \otimes{\underset{p}{\mu_{r}}}_{p} \otimes d x_{p}^{\nu_{1}} \otimes d x_{p}^{\nu_{2}} \cdots \otimes d x_{p}^{\nu_{s}}
$$

A escolha de um tensor do tipo $(r, s)$ em cada ponto $p \in \mathcal{M}$ de modo diferenciável, isto é, cujos componentes numa base coordenada são $\mathcal{C}^{\infty}$, define um campo tensorial $\mathcal{T}_{s}^{r}(\mathcal{M})$. Campos tensoriais são então objetos definidos na variedade e que induzem uma representação em cada sistema de coordenadas locais $\left(\mathcal{U}_{\alpha}, \Psi_{\alpha}\right)$ :

$$
T=T_{l m \cdots n}^{i j \cdots k}\left(x^{\mu}\right) \partial_{i} \otimes \partial_{j} \cdots \otimes \partial_{k} \otimes d x^{l} \otimes d x^{m} \cdots \otimes d x^{n}
$$


Observe que temos ainda a possibilidade de definir uma base não-coordenada $X_{i}$, $i=1, \ldots, N$ para o espaço tangente e a respectiva base dual $X^{k}$ satisfazendo $X^{k}\left(X_{i}\right)=\delta_{i}^{k}$. A base não-coordenada não satisfaz $X_{i}=\partial_{i}$ para todo $i$ e algum sistema local de coordenadas $x^{i}$. A caracterização da base coordenada é feita pelo teorema de Frobenius de Felice e Clarke (1990): Uma base é coordenada se e somente se $\left[X_{i}, X_{j}\right]=0$.

A transição entre coordenadas locais $\Psi_{\alpha} \circ \Psi_{\beta}^{-1}: \Psi_{\beta}\left(\mathcal{U}_{\alpha} \cap \mathcal{U}_{\beta}\right) \rightarrow \Psi_{\beta}\left(\mathcal{U}_{\alpha} \cap \mathcal{U}_{\beta}\right)$, doravante denotada apenas por $x \rightarrow \bar{x}$, induz uma regra de transformação para os componentes dos tensores entre os diversos sistemas de coordenadas:

$$
\bar{T}_{l m \cdots n}^{i j \cdots k}\left(\overline{x^{\mu}}\right)=\frac{\partial \bar{x}^{i}}{\partial x^{a}} \cdot \frac{\partial \bar{x}^{j}}{\partial x^{b}} \cdots \frac{\partial \bar{x}^{k}}{\partial x^{c}} \cdot \frac{\partial x^{c}}{\partial \bar{x}^{l}} \cdot \frac{\partial x^{d}}{\partial \bar{x}^{m}} \cdots \frac{\partial x^{e}}{\partial \bar{x}^{n}} T_{d e \cdots f}^{a b \cdots c}\left(x^{\mu}\right)
$$

A regra de transformação para uma transformação $X_{i} \rightarrow \bar{X}_{j}$ entre bases não-coordenadas é dada pela regra acima com a matriz da mudança de base $\Phi_{j}^{k}, \bar{X}=\Phi_{j}^{k} X_{k}$, no lugar de $\frac{\partial \bar{x}^{i}}{\partial x^{a}}$.

Podemos ainda expressar a medida de integração em termos das diferenciais:

$$
\int f d x^{1} \wedge d x^{2} \wedge \cdots \wedge d x^{N}=\int f d^{N} x
$$

onde $d x^{1} \wedge d x^{2} \wedge \cdots \wedge d x^{N}$ é o produto antissimetrizado das diferenciais, isto é, um tensor $\mathcal{T}_{N}^{0}(\mathcal{M})$ totalmente antissimétrico. A totalidade desses constitui o espaço vetorial das $N$-formas $\Omega_{N}(\mathcal{M})$.

Escrito dessa forma, a regra de transformação tensorial induz a regra de transformação da medida $d^{N} x=\left|\frac{\partial x}{\partial x^{\prime}}\right| d^{N} x^{\prime}$, sob a hipótese do determinante da diferencial da transformação de coordenas ser positivo. Recobrir a variedade $\mathcal{M}$ por um sistema de coordenadas tal que este determinante seja sempre positivo, quando possível, define uma orientação. A notação acima é útil, pois nos leva a uma regra para induzir uma medida em subvariedades: toda $k$-forma $\omega$ em $\mathcal{M}$ induz uma medida nas subvariedades $\mathcal{S}$ de dimensão $k \leq \operatorname{dim}(\mathcal{M})$, pois o mapa coordenado local, $\phi: \mathcal{V} \cap S \rightarrow \mathcal{U}$, sendo $\mathcal{U}$ um aberto de $\mathbb{R}^{k}$ e $\mathcal{V}$ um aberto de $\mathcal{M}$, induz em $\mathcal{U}$ uma $k$-forma $\omega^{\prime}$ dada por $\omega^{\prime}\left(v_{1}, \ldots, v_{k}\right)=\omega\left(d \phi^{-1}\left(v_{1}\right), \ldots, d \phi^{-1}\left(v_{k}\right)\right)=$ $\phi_{*} \omega\left(v_{1}, \ldots, v_{k}\right)$. A regra $\phi_{*}: \omega \rightarrow \omega^{\prime}$ é chamada de push-forward e sua definição é extensível a todos os tensores: Dado $\phi: \mathcal{M} \rightarrow \mathcal{N}$ um difeomorfismo, primeiro definimos $\phi_{*}: \mathcal{T}_{0}^{1}(\mathcal{M}) \rightarrow \mathcal{T}_{0}^{1}(\mathcal{N})$ dado por:

$$
\left(\phi_{*} X\right)(\phi(p))=d \phi_{p}(X(p))
$$


em seguida $\phi_{*}: \mathcal{T}_{1}^{0}(\mathcal{M}) \rightarrow \mathcal{T}_{1}^{0}(\mathcal{N})$ dado $\eta \in \Omega_{1}(\mathcal{M})$ e $v \in T_{p} \mathcal{M}:$

$$
\left(\left(\phi_{*} \eta\right)_{\phi(p)}, v\right)=\left(\eta_{p}, d \phi_{p}^{-1}(v)\right)
$$

e para para tensores em geral, definimos $\phi_{*}: \mathcal{T}_{s}^{r}(\mathcal{M}) \rightarrow \mathcal{T}_{s}^{r}(\mathcal{N})$ :

$$
\left(\phi_{*} T\right)_{\phi(p)}\left(\eta_{1}, \cdots, \eta_{r}, v_{1}, \cdots, v_{s}\right)=T\left(\phi^{*} \eta_{1}, \cdots, \phi^{*} \eta_{r}, \phi^{*} v_{1}, \cdots, \phi^{*} v_{s}\right)
$$

onde $\phi^{*}$ é a inversa de $\phi_{*}$, conhecida como pull-back.

Para definir a integral em $\mathcal{S}$, podemos usar uma partição da unidade $\Psi$, isto é, uma coleção de funções $\mathcal{C}^{\infty}$, denotadas $\psi_{\alpha}$, que se anulam fora de um dado compacto $K$ dentro de cada vizinhança coordenada $\mathcal{V}_{\alpha}$, coberta por um mapa $\phi_{\alpha}: \mathcal{V} \rightarrow \mathbb{R}^{k}$ descrita por coordenadas $x_{\alpha}^{i}$, de tal modo que cada ponto $p$ de $\mathcal{M}$ admite uma vizinhança no qual apenas um número finito de funções $\phi_{\alpha}$ é diferente de zero e $\sum_{\alpha} \phi_{\alpha}=1$.

Definimos então a integral de uma função $f$ na variedade $\mathcal{M}$ com respeito a medida $\omega \in \Omega_{N}(\mathcal{M}):$

$$
\int_{\mathcal{M}} f \omega=\sum_{\alpha} \int f \cdot \psi_{\alpha} \cdot \phi_{\alpha *} \omega
$$

onde a integral à direita é feita conforme 2.32 .

Outra maneira seria definir outro sistema de vizinhanças coordenadas $\mathcal{U}_{1}^{\prime}=\mathcal{U}_{1}, \mathcal{U}_{2}^{\prime}=$ $\mathcal{U}_{2}-\mathcal{U}_{1} \cap \mathcal{U}_{2}, \mathcal{U}_{3}^{\prime}=\mathcal{U}_{3}-\mathcal{U}_{3} \cap \mathcal{U}_{1}-\mathcal{U}_{3} \cap \mathcal{U}_{2}, \ldots$ todos disjuntos e somar as integrais no interior-maior subconjunto aberto- de cada $\mathcal{U}_{\alpha}^{\prime}$ :

$$
\sum_{\alpha} \int_{\operatorname{int}\left(\mathcal{U}_{\alpha}^{\prime}\right)} \phi_{*} f \cdot \omega
$$

Toda $k$-forma, num sistema local de coordenadas $x^{i}$, pode ser escrita da forma

$$
\omega_{\alpha \ldots \beta} \underbrace{d x^{\alpha} \wedge \ldots \wedge d x^{\beta}}_{k} .
$$

introduzimos então a derivada exterior $d: \Omega_{k}(\mathcal{M}) \rightarrow \Omega_{k+1}(\mathcal{M})$ :

$$
d \omega=\partial_{\sigma} \omega_{\alpha \ldots \beta} d x^{\sigma} \wedge d x^{\alpha} \wedge \ldots \wedge d x^{\beta}
$$

Usando essa notação, enunciamos o teorema de Stokes:

$$
\int_{\Omega} d \omega=\int_{\partial \Omega} \omega
$$


onde $\partial \Omega$ é a fronteira de uma variedade compacta orientada $\Omega$ cuja orientação é induzida de $\Omega$. Isto é, todo ponto $p$ de $\partial \Omega$ adimite um vizinhança $\mathcal{U}$ e um mapa $\phi: \mathcal{U} \rightarrow \mathcal{V} \subset$ $\mathbb{R}^{k}$ tal que $\phi(\mathcal{U} \cap(\Omega \cup \partial \Omega))=\mathcal{V} \cap\left\{x^{k} \geq 0\right\}$ e $\phi(p)=0$, sendo $\phi$ restrito a $\mathcal{U} \cap \Omega$ compatível com a orientação de $\Omega$ e $\phi$ restrito a $\mathcal{U} \cap \partial \Omega$ compatível com a orientação de $\partial \Omega$. Compatibilidade de um mapa $\phi$ com a orientação de $\mathcal{M}$ significa que o determinante da transição de coordenadas entre $\phi$ e qualquer um dos mapas que definem a orientação de $\mathcal{M}$ é positivo.

Do teorema de Stokes, deduzimos o teorema de Gauss:

$$
\int_{\partial \Omega} \sum_{i} P^{i} d x^{1} \wedge \cdots \wedge \widehat{d x^{i}} \cdots \wedge d x^{N}=\int_{\Omega} P^{i}{ }_{i} d x^{1} \wedge \ldots \wedge d x^{N}
$$

onde o termo $\widehat{d x^{i}}$ é omitido.

A métrica é o exemplo imediato de campo tensorial correspondente a uma observável física (no sentido de, num dado sistema de coordenadas e em cada ponto, ser unicamente determinado pela observação de trajetótias de partículas teste, veja análise na páginas 60-61 de Hawking e Ellis (1973)). Os campos de vetores tangentes são identificados com os campos tensoriais contravariantes $\mathcal{T}_{0}^{1}(\mathcal{M})$. Na literatura física, entretanto, mais importante que a interpretação geométrica é a regra de transformação desses objetos. A covariância geral é então implementada escrevendo-se o sistema de equações diferenciais da forma:

$$
T_{\theta \vartheta \ldots \rho}^{\phi \varphi \ldots \eta}\left(g_{\alpha \beta}, \Psi(x), \Phi(x)_{\tau v \ldots \omega}^{\alpha \beta \ldots \gamma}, \ldots\right)=0
$$

Cada $T_{\theta \vartheta \ldots \rho}^{\phi \varphi \ldots \eta}$ é uma equação diferencial parcial nos campos cuja regra de transformação é tensorial. A regra de transformação 2.31 assegura que as equações 2.42 são válidas num dado sistema de coordenadas se, e somente se, forem válidas em qualquer outro sob a mesma forma. Vale frisar que a covariância geral não é uma lei física, e sim uma escolha arbitrária de formulação. Doravante, usaremos a expressão tensor, ao invés de campo tensorial.

A mais simples equação diferencial parcial envolvendo um tensor $\partial_{\mu} \psi^{\alpha}=0$ é incompatível com o requerimento de covariância geral. A derivada parcial não preserva a natureza tensorial:

$$
\frac{\partial x^{\mu}}{\partial \bar{x}^{\nu}} \partial_{\mu}\left(\frac{\partial \bar{x}^{\beta}}{\partial x^{\alpha}} \psi^{\alpha}\right)=\frac{\partial x^{\mu}}{\partial \bar{x}^{\nu}} \frac{\partial \bar{x}^{\beta}}{\partial x^{\alpha}} \partial_{\mu} \psi^{\alpha}+\frac{\partial x^{\mu}}{\partial \bar{x}^{\nu}} \frac{\partial^{2} \bar{x}^{\beta}}{\partial x^{\mu} \partial x^{\alpha}} \psi^{\alpha}
$$


A derivada parcial $\partial_{\mu}$ usual é completamente definida por um conjunto de propriedades algébricas: a linearidade $\partial_{\mu}(f+g)=\partial_{\mu}(f)+\partial_{\mu}(g)$; a regra de Liebniz $\partial_{\mu}(f g)=\left(\partial_{\mu} f\right) g+$ $f\left(\partial_{\mu} g\right)$; e a propriedade $\partial_{\mu} x^{\nu}=\delta_{\nu}^{\mu}$. Definimos então a derivada covariante $\nabla_{X} Y$, $X, Y \in \mathcal{X}(\mathcal{M})$, por propriedades análogas, mais o requerimento de preservar a natureza tensorial:

1. $\nabla_{X} Y$ é um tensor em $X: \nabla_{f X+g Z} Y=f \nabla_{X} Y+g \nabla_{Z} Y$, onde $f, g \in \mathcal{C}^{\infty}(\mathcal{M})$

2. $\nabla_{X} Y$ é linear em $Y: \nabla_{X}(\alpha Y+\beta Z)=\alpha \nabla_{X} Y+\beta \nabla_{Z} Y$, onde $\alpha, \beta \in \mathbb{R}$

3. $\nabla_{X} Y$ obedece a regra de Liebniz em $Y: \nabla_{X} f Y=X^{\mu} \partial_{\mu} f Y+f \nabla_{X} Y, f \in \mathcal{C}^{\infty}(\mathcal{M})$

Observe que esse conjunto de propriedades é independente da escolha de uma base. A regra $\nabla: \mathcal{X}(\mathcal{M}) \times \mathcal{X}(\mathcal{M}) \rightarrow \mathcal{X}(\mathcal{M})$ é chamada de conex $\tilde{a} \boldsymbol{o}$. Escolhendo uma base em cada $T_{p} \mathcal{M}$ de maneira suave $\left(\mathcal{C}^{\infty}\right)$, definimos uma base em $\mathcal{X}(\mathcal{M})$ denotada $X_{\tau}, \tau=1, . ., N$, a derivada covariante é então expressa nessa base por:

$$
\begin{aligned}
\nabla_{x^{\alpha} X_{\alpha}}\left(y^{\beta} X_{\beta}\right) & =\left(x^{\alpha} X_{\alpha} y^{\beta}\right) X_{\beta}+x^{\alpha} y^{\beta} \nabla_{X_{\alpha}} X_{\beta} \\
& =\left(x^{\alpha} X_{\alpha} y^{\tau}\right) X_{\tau}+x^{\alpha} y^{\beta} \Gamma_{\alpha \beta}^{\tau} X_{\tau}
\end{aligned}
$$

Dado um sistema de coordenadas locais, escolhamos a base coordenada correspondente para o espaço tangente $X_{\alpha}=\partial_{\alpha}, 2.44$ então se escreve como:

$$
\nabla_{x^{\alpha} \partial_{\alpha}} y^{\beta} \partial_{\beta}=\left(x^{\alpha} \partial_{\alpha} y^{\tau}+x^{\alpha} y^{\beta} \Gamma_{\alpha \beta}^{\tau}\right) \partial_{\tau}
$$

donde concluímos a regra de transformação tensorial com respeito ao índice $\tau$ da independência do conceito de derivada com respeito à escolha de base e da regra de transformação covariante da base $\partial_{\tau}$. Identicamente, a regra de transformação tensorial em $\alpha$ decorre da propriedade (1).

A derivada covariante pode ser estendida a todo campo tensorial $\mathcal{T}_{s}^{r}(\mathcal{M})$ :

1. Se $T$ é um tensor do tipo $\mathcal{T}_{s}^{r}(\mathcal{M}), \nabla T$ é um tensor do tipo $\mathcal{T}_{s+1}^{r}(\mathcal{M})$

2. $\nabla$ é linear e comuta com as contrações: $\nabla\left(A^{i} B_{i}\right)=(\nabla A)^{i} B_{i}+A^{i}(\nabla B)_{i}$

3. $\nabla$ obedece a regra de Leibniz: $\nabla(T \otimes R)=(\nabla T) \otimes R+T \otimes(\nabla R)$

4. $\nabla f=d f$ para $f \in \mathcal{C}(\mathcal{M})$ 
onde as propriedades acima contém as anteriores como caso particular, considerando-se adicionalmente (2) temos que:

$$
\nabla_{X^{\alpha} \partial_{\alpha}} A_{\beta} d x^{\beta}=\left(X^{\alpha} \partial_{\alpha} A_{\beta}-X^{\alpha} \Gamma_{\alpha \beta}^{\gamma} A_{\gamma}\right) d x^{\beta}
$$

Doravante, empregaremos a notação $T_{\theta \rho \cdots \sigma ; \tau}^{\alpha \beta \cdots \gamma}$ para os componentes da derivada covariante $\nabla_{X_{\tau}} T_{\theta \rho \cdots \sigma}^{\alpha \beta \cdots \gamma} X_{\alpha} \otimes \ldots \otimes X^{\sigma}$. Concluímos das propriedades acima que:

$$
T_{\theta \rho \cdots \sigma ; \tau}^{\alpha \beta \cdots \gamma}=T_{\theta \rho \cdots \sigma, \tau}^{\alpha \beta \cdots \gamma}+\Gamma_{\mu \tau}^{\alpha} T_{\theta \rho \cdots \sigma}^{\mu \beta \cdots \gamma}+\ldots-\Gamma_{\sigma \tau}^{\mu} T_{\theta \rho \cdots \mu}^{\mu \beta \cdots \gamma}
$$

onde $T_{\theta \rho \cdots \sigma, \tau}^{\alpha \beta \cdots \gamma}$ denota $\partial_{\tau} T_{\theta \rho \cdots \sigma}^{\alpha \beta \cdots \gamma}$.

A especificação de uma regra de derivação covariante define uma regra de transporte de vetores ao longo de uma curva $\gamma(t)$. Um campo vetorial $V(t)$ ao longo de $\gamma$ é paralelamente transportado se:

$$
\nabla_{\frac{d \gamma}{d t}(t)} V(t)=0
$$

onde $\nabla_{\frac{d \gamma}{d t}(t)}$ é definido através de qualquer extensão do campo vetorial $\frac{d \gamma(t)}{d t}$ para alguma vizinhança da curva $\gamma$. Isto define um mapa linear entre os espaços tangentes $T_{\gamma\left(t_{i}\right)} \mathcal{M}$ e $T_{\gamma\left(t_{f}\right)} \mathcal{M}$ dependente do caminho $\gamma(t):\left(t_{i}, t_{f}\right) \rightarrow \mathcal{M}:$

$$
\Gamma\left(\gamma(t), t_{i}, t_{f}\right) V\left(t_{i}\right)=V\left(t_{f}\right)
$$

reciprocamente, a derivada covariante pode ser reescrita através do transporte paralelo associado:

$$
\begin{aligned}
& \nabla_{\frac{d \gamma}{d t}} T\left(\gamma\left(t_{i}\right)\right)=\lim _{\delta t \rightarrow 0} \frac{1}{\delta t}\left\{T_{\epsilon \phi \cdots \gamma}^{\alpha \beta \cdots \chi}\left(\gamma\left(t_{i}+\delta t\right)\right) \Gamma^{-1}\left(\gamma, t_{i}, t_{i}+\delta t\right) \underset{\gamma\left(t_{i}+\delta t\right)}{X_{\alpha}} \otimes \ldots \otimes \Gamma^{-1}\left(\gamma, t_{i}, t_{i}+\delta t\right) \underset{\gamma\left(t_{i}+\delta t\right)}{X^{\gamma}}\right. \\
& \left.-T_{\epsilon \phi \cdots \gamma}^{\alpha \beta \cdots \chi}\left(\gamma\left(t_{i}\right)\right) \underset{\gamma\left(t_{i}\right)}{X_{\gamma}} \otimes \cdots \otimes \underset{\gamma\left(t_{i}\right)}{X^{\gamma}}\right\}
\end{aligned}
$$
onde $\underset{\gamma\left(t_{i}\right)}{X_{\gamma}}$ e $\underset{\gamma(t)}{X^{\gamma}}$ são uma base no espaço tangente e cotangente em $T_{\gamma(t)} \mathcal{M}$, respectivamente.

Deduzimos a regra de transformação dos símbolos $\Gamma_{\alpha \beta}^{\tau}=g^{\tau \sigma}\left\langle\nabla_{X_{\alpha}} X_{\beta}, X_{\sigma}\right\rangle$, os coeficientes da conexão pelas propriedades (1), (2) e (3):

$$
\bar{\Gamma}_{\alpha \beta}^{\tau}=\frac{\partial \bar{x}^{\tau}}{\partial x^{\tau^{\prime}}} \frac{\partial x^{\alpha^{\prime}}}{\partial \bar{x}^{\alpha}} \frac{\partial x^{\beta^{\prime}}}{\partial \bar{x}^{\beta}} \Gamma_{\alpha^{\prime} \beta^{\prime}}^{\tau^{\prime}}+\frac{\partial \bar{x}^{\tau}}{\partial x^{\tau^{\prime}}} \frac{\partial^{2} x^{\tau^{\prime}}}{\partial \bar{x}^{\alpha} \partial \bar{x}^{\beta}}
$$

Numa teoria em que é válido o princípio da equivalência, $\Gamma_{\alpha \beta}^{\tau}$ deve se anular em cada ponto sob uma conveniente transformação de coordenadas adaptada ao ponto em questão. Portanto:

$$
\Gamma_{\alpha \beta}^{\tau}=\Gamma_{\beta \alpha}^{\tau}
$$


Isso se deve ao fato de que $\Gamma_{\alpha \beta}^{\tau}-\Gamma_{\beta \alpha}^{\tau}$, por 2.51, é um tensor, que, portanto, é identicamente nulo se se anular em qualquer sistema de coodenadas. A conexão satisfazendo essa propriedade é dita simétrica. Uma vez sabido que $\Gamma_{\alpha \beta}^{\tau}$ é identicamente zero em $p$ para algum sistema de coordenadas local, no qual a métrica é dada por $\eta_{\alpha \beta}$ no mesmo ponto, a diferencial da mudança de coordenadas entre o referencial localmente inercial e o referencial laboratório determina $\Gamma_{\alpha \beta}^{\tau}$ por 2.51 .

Uma maneira mais conveniente é observar que no referencial localmente inercial:

$$
\partial_{\mu}\langle X, Y\rangle=\left\langle\partial_{\mu} X, Y\right\rangle+\left\langle X, \partial_{\mu} Y\right\rangle, \quad X, Y \in \mathcal{X}(\mathcal{M})
$$

que se escreve de modo geralmente covariante da forma:

$$
\nabla_{\mu}\left(g_{\alpha \beta} X^{\alpha} X^{\beta}\right)=g_{\alpha \beta} \nabla_{\mu}\left(X^{\alpha}\right) X^{\beta}+g_{\alpha \beta} X^{\alpha} \nabla_{\mu}\left(X^{\beta}\right)
$$

empregamos aqui a notação de Einstein de soma de índices repetidos. A equação 2.54 equivale então à equação:

$$
\nabla_{\mu} g_{\alpha \beta}=0
$$

que juntamente com o requerimento de simetria da conexão determina unicamente os coeficientes da conexão:

$$
\Gamma_{\alpha \beta}^{\mu}=\frac{1}{2} g^{\mu \tau}\left(\partial_{\alpha} g_{\tau \beta}+\partial_{\beta} g_{\alpha \tau}-\partial_{\tau} g_{\alpha \beta}\right)
$$

a conexão acima é chamada de conexão de Levi-Civita, onde mais uma vez recuperamos os símbolos de Christoffel 2.26.

O transporte associado à métrica é dito paralelo devido ao fato de que, ao mergulhar isometricamente uma variedade $\mathcal{M}$ num espaço euclidiano $\mathbb{R}^{n}$, cada ponto $p$ de cada curva $\gamma(t)$ admite uma vizinhança $\mathcal{V}$ tal que existe uma superfície plana (isométrica ao plano) que contém a curva em $\mathcal{V}$ e cujo espaço tangente em cada ponto $\gamma(t) \in \mathcal{V}$ é paralelo ao espaço tangente de $\mathcal{M}$ em $\gamma(t)$. Nesta situação, a derivada covariante é a derivada usual projetada no plano tangente. O transporte paralelo no plano é, portanto, o mesmo que o da superfície. Mas o plano adimite um sistema de coordenadas tal que $\frac{d V(t)}{d t}=0$, isto é, um campo vetorial constituído de vetores paralelos ao longo de uma curva.

Nessa formulação, a geodésica é a curva tal que o vetor tangente em cada ponto é paralelamente transportado:

$$
\frac{d \gamma(t)}{d t}=\Gamma\left(\gamma(t), t_{i}, t\right) \frac{d \gamma\left(t_{i}\right)}{d t}
$$


observe que a definição acima é dependente da parametrização da curva. Quando a parametrização é tal que 2.57 é válida, a mesma é dita $\boldsymbol{a}$ fim.

\subsubsection{As equações de campo}

Uma vez tendo promovido a métrica ao status de campo físico, necessitamos de uma lei física local que a determine, isto é, uma equação de campo. O princípio da equivalência nada nos diz sobre a forma da equação de campo, apenas nos diz sobre como a gravidade afeta a dinâmica dos sistemas físicos. A equação é então postulada através de um conjunto de requerimentos: localidade; covariância geral e limite newtoniano, ou seja, as equações linearizadas em $h_{\alpha \beta}$, onde $g_{\alpha \beta}=\eta_{\alpha \beta}+h_{\alpha \beta}$, se reduzem à equação de Poisson no limite de campo fraco, i.e. $\left|h_{\alpha \beta}\right|<<1$, estacionário, i.e. $\frac{\partial}{\partial t} h_{\alpha \beta}=0$ e velocidades não relativísticas $v<<c$ :

$$
\Delta \Phi=4 \pi G \rho
$$

O limite acima é obtido da equação da geodésica:

$$
\frac{d^{2} x^{\mu}}{d s^{2}}+\Gamma_{\alpha \beta}^{\mu} \frac{d x^{\alpha}}{d s} \frac{d x^{\beta}}{d s}=0
$$

fazendo $d s^{2}=c^{2} d t^{2}, \frac{d x^{i}}{d s}=0, i=1, . .3$ e $\Gamma_{00}^{i}=\frac{1}{2} \eta^{i j} \frac{h_{00}}{\partial x^{j}}=-\frac{1}{2} \frac{\partial h_{00}(x)}{\partial x^{i}}$ :

$$
\begin{aligned}
\frac{d^{2} x^{\mu}}{d x^{2}} & =\frac{1}{2} \frac{\partial h_{00}(x)}{\partial x^{i}} \frac{d x^{0}}{d t} \frac{d x^{0}}{d t} \\
\frac{d^{2} x^{0}}{d t^{2}} & =0
\end{aligned}
$$

O que nos conduz ao limite de campo fraco

$$
g_{00}=1-2 \Phi(x)
$$

onde $\Phi(x)$ é o potencial Newtoniano.

Primeiramente, consideremos que, no referencial localmente inercial, o campo gravitacional depende da densidade de energia de um conjunto de partículas em repouso. Um gás de partículas com pressão nula e sem graus de liberdade internas, por exemplo, é, localmente e neste referencial, completamente descrito por sua densidade de energia $\rho$, e por isso, nenhuma informação adicional pode então influenciar o campo gravitacional. Num referencial em movimento uniforme em relação a este, esta mesma distribuição de 
matéria apresentará fluxo de energia, densidade de momento não nula e fluxo de momento em correspondência biunívoca com a densidade de energia no referencial anterior. No referencial Lorentz transformado, concluímos, portanto, que o campo gravitacional depende não apenas da densidade de energia, mas também da densidade de momento e do fluxo dessas quantidades, uma vez que estas últimas são completamente definidas pela densidade de energia no primeiro referencial, que por hipótese determina o campo gravitacional. Em outras palavras, a invariância local de Lorentz nos leva a considerar não apenas a densidade de energia como fonte do campo gravitacional.

A situação acima sugere que estas quantidades (densidade de energia, momento e o fluxo destas) transformam-se conjuntamente via transformações de Lorentz. De fato, elas determinam o tensor energia-momento, que para um dado conjunto de partículas se escreve:

$$
T^{\mu \nu}=\sum_{N} \int p_{N}^{\mu} \frac{d x_{N}^{\nu}\left(t^{\prime}\right)}{d t} \delta^{4}\left(x-x_{N}\left(t^{\prime}\right)\right) d t^{\prime}=\sum_{N} \int p_{N}^{\mu} \frac{d x_{N}^{\nu}(s)}{d s} \delta^{4}\left(x-x_{N}(s)\right) d s
$$

donde deduzimos da definição de quadrimomento relativístico $p^{\mu}=m \frac{d x^{\mu}}{d s}$, onde $s$ é comprimento relativisticamente invariante da linha de mundo descrita pela partícula, bem como de sua regra de transformação contravariante e da invariância da delta de Dirac- consequência da invariância do elemento de quadrivolume $d^{4} x$ por transformações de Lorentz-, a regra de transformação do tensor energia-momento:

$$
T^{\alpha \beta}=\Lambda_{\mu}^{\alpha} \Lambda_{\nu}^{\beta} T^{\mu \nu}
$$

Assumindo esta como uma definição no referencial localmente inercial e promovendo ao status de tensor por transformações gerais de coordenadas, não apenas de Lorentz, este é um tensor bem definido.

O Tensor energia-momento 2.60 satisfaz ainda, sob a hipótese de conservação do quadrimomento total, a lei de conservação:

$$
\partial_{\beta} T^{\alpha \beta}=0
$$

que se estende de pelo princípio da covariância geral da forma:

$$
T_{; \beta}^{\alpha \beta}=0
$$


O raciocínio acima nos leva a uma equação da foma:

$$
G^{\alpha \beta}=\kappa T^{\alpha \beta}
$$

no qual $G^{\alpha \beta}$ deve ser um tensor com dependência local em $g_{\mu \nu}$, simétrico e conservado no sentido $G_{; \beta}^{\alpha \beta}=0$. A equação de Poisson sugere uma equação diferencial parcial de segunda ordem no campo. Mais ainda, não existe tensor que possa ser construído apenas da métrica e de suas derivadas primeiras que não seja função apenas do tensor métrico em si, não de suas derivadas. Isso porque sempre existe uma mudança de coordenadas em torno de $p$ tal que $g_{\alpha \beta}=\eta_{\alpha \beta}$ e $\partial_{\mu} g_{\alpha \beta}=0$ em $p$. De fato, construímos esse sistema de coordenadas através das geodésicas que partem de $p$ com vetor tangente dentro de um dada vizinhança $\mathcal{V}$ da origem do espaço tangente em $p$ e são definidas para valores do seu parâmetro afim $\lambda \in(-1,1)$. Associamos a cada $v \in T_{p} \mathcal{M}$ o ponto $\exp _{p}(v)=\gamma(1, v, p)$, onde $\gamma(\lambda, v, p)$ é a geodésica que satisfaz $\left.\frac{d \gamma(\lambda, v, p)}{d \lambda}\right|_{\lambda=\lambda_{0}}=v \in \mathcal{V}$ e $\gamma\left(\lambda_{0}\right)=p$. Este é chamado mapa exponencial: $\exp : \mathcal{V} \subset T_{p} \mathcal{M} \rightarrow \mathcal{M}$. Uma vez que as geodésicas que emanam de um mesmo ponto de um espaço curvo podem se encontrar, esta correspondência não se estende necessariamente de maneira inversível a todo espaço tangente, mas podemos garantir pelo teorema da função inversa que sempre existe uma vizinhança em torno da origem tal que o mapa exponencial define um difeomorfismo. A equação da geodésica nesse sistema de coordenadas se escreve como

$$
\frac{d^{2} x^{\mu}}{d s^{2}}=0
$$

o que implica $\Gamma_{\alpha \beta}^{\mu}=0$ e consequentemente $\partial_{\mu} g_{\alpha \beta}=0$.

A construção de tensores com as propriedades de $G^{\alpha \beta}$ pode se dar por intermédio de funcionais diferenciáveis invariantes por transformações gerais de coordenadas. Dado um campo vetorial $\xi^{\alpha}$ diferenciável, definimos uma família de um parâmetro de transformações que se escreve da forma $\bar{x}^{\mu}=x^{\mu}+\epsilon \cdot \xi^{\mu}+\mathcal{O}\left(\epsilon^{2}\right)$. Supondo ainda que $\xi$ se anula fora de algum compacto $K$, a invariância do funcional $S$ se escreve como:

$$
\delta S=S\left[\frac{\partial x^{\alpha}}{\partial \bar{x}^{\mu}} \frac{\partial x^{\beta}}{\partial \bar{x}^{\nu}} g_{\alpha \beta}(x(\bar{x}))\right]-S\left[g_{\mu \nu}(\bar{x})\right]=0,
$$

da diferenciabilidade decorre que

$$
\delta S=\left\langle\frac{\delta S}{\delta g_{\alpha \beta}}, \mathcal{L}_{\xi} g_{\alpha \beta}\right\rangle \cdot \epsilon+\mathcal{O}\left(\epsilon^{2}\right)=\int\left(\frac{1}{\sqrt{-g}} \frac{\delta S}{\delta g_{\alpha \beta}}\right) \mathcal{L}_{\xi} g_{\alpha \beta} \sqrt{-g} d^{4} x \cdot \epsilon+\mathcal{O}\left(\epsilon^{2}\right)
$$


na expressão acima, $\mathcal{L}_{\xi} g_{\alpha \beta}$ é a chamada derivada de Lie:

$$
\mathcal{L}_{\xi} g_{\alpha \beta}=\lim _{\epsilon \rightarrow 0} \frac{1}{\epsilon}\left\{\frac{\partial x^{\alpha}}{\partial \bar{x}^{\mu}} \frac{\partial x^{\beta}}{\partial \bar{x}^{\nu}} g_{\alpha \beta}(x(\bar{x}))-g_{\mu \nu}(\bar{x})\right\}
$$

que se reduz a

$$
\mathcal{L}_{\xi} g_{\alpha \beta}=\xi^{\mu} g_{\alpha \beta, \mu}+g_{\mu \beta} \xi_{, \alpha}^{\mu}+g_{\alpha \mu} \xi_{, \beta}^{\mu}=\xi_{\alpha ; \beta}+\xi_{\alpha ; \beta}=\xi_{(\alpha ; \beta)}
$$

sendo a regra $\xi^{\alpha} \rightarrow \xi_{\alpha}$ dada por $\xi_{\beta}=g_{\alpha \beta} \xi^{\alpha}$. Escrevendo a integral em 2.67 em termos da medida $\sqrt{-g} d^{4} x$, invariante por transformações gerais de coordenas e igual a $d^{4} x$ em $p$ no referencial localmente inercial em $p$, asseguramos a regra de transformação tensorial da quantidade $T^{\alpha \beta}=\frac{1}{\sqrt{-g}} \frac{\delta S}{\delta g_{\alpha \beta}}$ dada a natureza escalar de $S$.

Temos então que:

$$
\begin{gathered}
\delta S=\int T^{\alpha \beta} \xi_{(\alpha ; \beta)} \sqrt{-g} d^{4} x \cdot \epsilon+\mathcal{O}\left(\epsilon^{2}\right) \\
=\left(\int\left(T^{\alpha \beta} \xi_{\alpha}\right)_{; \beta} \sqrt{-g} d^{4} x-\int T_{; \beta}^{\alpha \beta} \xi_{\alpha} \sqrt{-g} d^{4} x\right) \cdot \epsilon+\mathcal{O}\left(\epsilon^{2}\right) \\
=\left(\int \frac{1}{\sqrt{-g}} \frac{\partial\left(\sqrt{-g} T^{\alpha \beta} \xi_{\alpha}\right)}{\partial x^{\beta}} \sqrt{-g} d^{4} x-\int T_{; \beta}^{\alpha \beta} \xi_{\alpha} \sqrt{-g} d^{4} x\right) \cdot \epsilon+\mathcal{O}\left(\epsilon^{2}\right)
\end{gathered}
$$

Aplicando o teorema de Gauss $\int_{\Omega} P^{\mu}{ }_{, \mu} d^{4} x=\int_{\partial \Omega} P^{\mu} d \sigma_{\mu}$, mais o fato de $\xi=0$ fora de algum compacto $K$ e fazendo $\delta S=0$ em primeira ordem em $\epsilon$ :

$$
\int T_{; \beta}^{\alpha \beta} \xi_{\alpha} \sqrt{-g} d^{4} x=0
$$

Pela arbitrariedade de $\xi$ e simetria de $\xi_{(\alpha ; \beta)}$ nos índices $\alpha$ e $\beta$, temos que:

$$
T^{\alpha \beta}=\frac{1}{\sqrt{-g}} \frac{\delta S}{\delta g_{\alpha \beta}}
$$

é um tensor simétrico conservado.

Reciprocamente, podemos reconstruir o funcional pela condição inicial $S[0]=0$ dado $T^{\alpha \beta}(x)\left[g_{\mu \nu}\right]$ satisfazendo

$$
\frac{\delta \sqrt{-g} T^{\alpha \beta}}{\delta g_{\mu \nu}}=\frac{\delta \sqrt{-g} T^{\mu \nu}}{\delta g_{\alpha \beta}}
$$

que equivale a 2.71 . 
De fato, dada uma família de um parâmetro $g_{\mu \nu}^{\lambda}(x)$ tal que $g_{\mu \nu}^{1}(x)=g_{\mu \nu}(x)$, defina:

$$
S\left[g_{\alpha \beta}\right]=\int_{0}^{1} d \lambda \int \sqrt{-g} d^{4} x \sum_{\alpha \beta} T^{\alpha \beta}\left[g_{\mu \nu}^{\lambda}\right] \frac{d g_{\alpha \beta}^{\lambda}(x)}{d \lambda}
$$

A independência de 2.73 com respeito a família $g_{\alpha \beta}^{\lambda}$ segue da condição 2.72 , como podemos constatar variando o caminho, mantendo-se fixo os extremos e aplicando 2.72 .

Invertendo a ordem das integrais acima, e assumindo $T^{\alpha \beta}$ uma função local em $g_{\mu \nu}$, reescrevemos o funcional na forma:

$$
S=\int \mathcal{L}\left[g_{\alpha \beta}\right] \sqrt{-g} d^{4} x
$$

onde $\mathcal{L}$ é um escalar construído como função local da métrica.

O requerimento de que sistemas de equações diferenciais locais nos campos são deriváveis por funcionais da forma 2.74 através do requerimento $\frac{\delta S}{\delta \phi}=0$ para algum $\mathcal{L}$, escalar e função local dos campos envolvidos no processo físico, é um postulado na física. Tais funcionais definem a ação dos campos e $\mathcal{L}$ a densidade lagrangiana. Podemos usar esse postulado para definir o tensor energia-momento associado a um conjunto de campos a partir de uma ação compatível com o princípio da covariância geral:

$$
S_{M}=\int \mathcal{L}\left(g_{\alpha, \beta}, \Gamma_{\alpha \beta}^{\mu}, \phi, \partial \phi\right) \sqrt{-g} d^{4} x
$$

sendo $\phi$ um campo tensorial. As equações da dinâmica são postuladas da forma:

$$
\frac{\delta S_{M}}{\delta \phi}=0
$$

Estas equações são automaticamente tensoriais. E nos levam a uma definição para o tensor energia-momento dos campos:

$$
T_{M}^{\alpha \beta}=\frac{1}{\sqrt{-g}} \frac{\delta S_{M}}{\delta g_{\alpha \beta}}
$$

Conforme dito, para construir $\mathcal{L}\left[g_{\alpha \beta}\right]$, necessitamos considerar termos além da derivada de primeira ordem, de modo a produzir um escalar não trivial, isto é, não constante. Para produzir uma equação de segunda ordem, $\mathcal{L}$ deve ser tal que $\mathcal{L}=\mathcal{L}\left(g_{\alpha \beta}, \partial g_{\alpha \beta}, \partial^{2} g_{\alpha \beta}\right)$ e:

$$
\frac{\partial \mathcal{L}}{\partial\left(\partial_{\mu \nu} g_{\alpha \beta}\right)}=w^{\alpha \beta \nu \mu}\left(g_{\alpha \beta}\right)
$$

conforme deduzimos das equações de Euler-lagrange:

$$
\frac{\delta S}{\delta g_{\alpha \beta}}=\frac{\partial \mathcal{L}}{\partial g_{\alpha \beta}}-\partial_{\mu} \frac{\partial \mathcal{L}}{\partial\left(\partial_{\mu} g_{\alpha \beta}\right)}+\partial_{\mu \nu} \frac{\partial \mathcal{L}}{\partial\left(\partial_{\mu \nu} g_{\alpha \beta}\right)}=0
$$


O que resulta numa ação da forma:

$$
S=\int G\left(g_{\alpha \beta}, \partial g_{\alpha \beta}\right) \sqrt{-g} d^{4} x+\int w^{\alpha \beta \nu \mu}(g) \frac{\partial^{2} g_{\alpha \beta}}{\partial x^{\nu} \partial x^{\mu}} \sqrt{-g} d^{4} x
$$

de modo que:

$$
\begin{aligned}
S= & \int G\left(g_{\alpha \beta}, \partial g_{\alpha \beta}\right) \sqrt{-g} d^{4} x+\int \frac{\partial}{\partial x^{\nu}}\left(w^{\alpha \beta \nu \mu}(g) \frac{\partial g_{\alpha \beta}}{\partial x^{\mu}} \sqrt{-g}\right) d^{4} x \\
& -\int \frac{\partial}{\partial x^{\nu}}\left(w^{\alpha \beta \nu \mu}(g) \sqrt{-g}\right) \frac{\partial g_{\alpha \beta}}{\partial x^{\mu}} d^{4} x
\end{aligned}
$$

onde o segundo termo acima se reduz a uma integral na fronteira $\partial \Omega$ que se anula para variações de $g_{\alpha \beta}$ de suporte compacto, produzindo uma ação invariante por transformações gerais de coordenadas que depende efetivamente das derivadas até primeira ordem.

No referencial localmente inercial em $p$, o termo $w^{\alpha \beta \nu \mu}(g) \frac{\partial^{2} g_{\alpha \beta}}{\partial x^{\nu} \partial x^{\mu}}$ em 2.80 se reduz a uma combinação linear a coeficientes constantes. Esta condição implica na existência de um único escalar $R$, a menos de uma constante multiplicativa, o escalar de curvatura:

$$
R=g^{\alpha \beta} R_{\alpha \beta}
$$

onde

$$
R_{\alpha \beta}=R_{\alpha \mu \beta}^{\mu}
$$

é o tensor de Ricci, e

$$
R_{\beta \mu \nu}^{\alpha}=\frac{\partial \Gamma_{\beta \mu}^{\alpha}}{\partial x^{\nu}}-\frac{\partial \Gamma_{\beta \nu}^{\alpha}}{\partial x^{\mu}}+\Gamma_{\beta \mu}^{\eta} \Gamma_{\nu \eta}^{\alpha}-\Gamma_{\beta \nu}^{\eta} \Gamma_{\mu \eta}^{\alpha}
$$

\section{é tensor de curvatura de Riemann-Christoffel.}

As equações de campo da matéria mais campo gravitacional são portando postuladas pelo requerimento de que a seguinte ação seja estacionária:

$$
S=\int \frac{1}{\kappa} R \sqrt{-g} d^{4} x+\int \mathcal{L}_{M} \sqrt{-g} d^{4} x
$$

onde $\kappa$ é fixado pelo limite de campo fraco 2.59 reproduzindo a equação de Poisson 2.58 como sendo $8 \pi G$, produzindo as equações de Einstein:

$$
\begin{aligned}
G^{\alpha \beta} & =8 \pi G T^{\alpha \beta} \\
R^{\alpha \beta}-\frac{1}{2} R g^{\alpha \beta} & =8 \pi G T^{\alpha \beta}
\end{aligned}
$$


onde $G^{\alpha \beta}$ é o tensor de Einstein.

O requerimento de linearidade com respeito às derivadas de segunda ordem no referencial localmente inercial, juntamente com a simetria e conservação do tensor de Einstein, são suficientes para alternativamente levar às equações de Einstein de modo único. Tal linearidade pode ser justificada pela invariância de escala. De fato, $G_{\mu \nu}$ deve ter dimensão de comprimento elevado a menos dois, $L^{-2}$. Supondo ainda que seja analítico nas derivadas de $g_{\mu \nu}$, decorre que não deve haver nenhuma constante dimensional (com dimensão de comprimento elevado a alguma potência) que multiplique um monômio nas derivadas da métrica. Os únicos monômios de dimensão $L^{-2}$ associados a derivadas de ordem segunda são lineares.

O tensor de Riemann está intimamente relacionado ao conceito de transporte paralelo. Dado um vetor $u_{p}$ em $p$, as curvas integrais associadas a dois campos vetoriais $X$ e $Y$, podemos transportar $u_{p}$ ao longo de $\gamma_{1}(t)$, a curva que passa por $p$ e cujo vetor tangente em cada ponto é $X\left(\gamma_{1}(t)\right)$, entre $t_{0}$ e $t_{1}=t_{0}+\delta t$ e em seguida transportar ao logo da curva integral $\gamma_{2}(s)$, a curva que passa por $\gamma_{1}\left(t_{1}\right)$ e cujo vetor tangente em cada ponto é $Y\left(\gamma_{2}(s)\right)$, entre $s_{0}$ e $s_{1}=s_{0}+\delta s$, obtendo o vetor.

$$
u_{X Y}=\Gamma\left(s_{0}, s_{1}, \gamma_{2}\right) \circ \Gamma\left(t_{0}, t_{1}, \gamma_{1}\right) u_{p}
$$

Reciprocamente, podemos transportar $u_{p}$ através a curva $\gamma_{1}^{\prime}(t)$, a curva que passa por $p$ e cujo vetor tangente em cada ponto é $Y\left(\gamma_{1}^{\prime}(t)\right)$, entre $t_{0}$ e $t_{1}=t_{0}+\delta t$ e em seguida transportar ao longo de $\gamma_{2}^{\prime}$, a curva que passa por $\gamma_{2}^{\prime}\left(t_{1}\right)$ e cujo vetor tangente em cada ponto é $Y\left(\gamma_{2}^{\prime}(s)\right)$, entre $s_{0}$ e $s_{1}=s_{0}+\delta s$, obtendo o vetor:

$$
u_{Y X}=\Gamma\left(s_{0}, s_{1}, \gamma_{2}^{\prime}\right) \circ \Gamma\left(t_{0}, t_{1}, \gamma_{1}^{\prime}\right) u_{p}
$$

A diferença entre esses vetores é:

$$
u_{X Y}-u_{Y X}=R_{\beta \mu \nu}^{\alpha} X^{\mu} Y^{\nu} u^{\beta} e_{\alpha} \delta t \delta s+\mathcal{O}\left(\delta t^{3}, \delta s^{3}\right)
$$

onde $X^{\mu}$ e $Y^{\nu}$ são os componentes de $X$ e $Y$ na base $e_{\beta}$, donde concluímos que $R_{\beta \mu \nu}^{\alpha}$ é um tensor, visto que $u_{X Y}-u_{Y X}$ é um vetor e da regra de transformação dos componentes de $X, Y$ e $u_{p}$. Donde concluímos que o transporte paralelo é independente da trajetória se e somente se

$$
R_{\beta \mu \nu}^{\alpha}=0
$$


No referencial localmente inercial, podemos eleger uma base $X_{i}, i=1, \ldots, N$ para o espaço tangente em $p$, de tal modo que $<X_{j}, X_{k}>_{p}=\eta_{j k}$. Transportando esta base paralelamente para todos os pontos de uma vizinhança $\mathcal{V}$ de $p$ obtemos um conjunto campo vetoriais $X_{i}$ tais que

$$
\left[X_{i}, X_{j}\right]=\nabla_{X_{i}} X_{j}-\nabla_{X_{j}} X_{i}=0
$$

que pelo teorema de Frobenius implica na integrabilidade local do sistema de coordenadas tal que $g_{\alpha \beta}=\eta_{\alpha \beta}$, isto é, $X_{i}=\partial_{i}$. O que nos leva a famosa conclusão de que, na presença de matéria, não existe sistema de coordenadas tal que $g_{\alpha \beta}=\eta_{\alpha \beta}$ em todos os pontos de uma dada vizinhança, ou, equivalentemente, o espaço-tempo é curvo.

Por fim, o tensor de Riemann possui as seguintes simetrias:

$$
\begin{gathered}
R_{i j k l}=-R_{i j l k} \\
R_{i j k l}=-R_{j i l k} \\
R_{i j k l}=R_{k l i j} \\
R_{i j k l}+R_{i l j k}+R_{i k l j}=0
\end{gathered}
$$

e a identidade $\boldsymbol{d e}$ Bianchi, da qual se deduz a conservação do tensor de Einstein:

$$
R_{i j k l ; m}+R_{i j m k ; l}+R_{i j l m ; k}=0
$$

das simetrias resultam que existem 20 componentes independentes no tensor de Riemann em quatro dimensões. 
Capítulo 3

\section{Cosmologia inflacionária}

\subsection{Introdução}

Conforme dito, a formulação local da física clássica é implementada através do conceito de campo, enquanto leis físicas locais são equações diferenciais parciais envolvendo derivadas no mesmo ponto. Há ainda outro requerimento na estrutura dessas equações: o caráter hiperbólico, isto é, a possibilidade de formular localmente, ou seja, na vizinhança de cada ponto $p$, o problema de valor inicial em função da informação definida na hipersuperfície que passa por $p$.

Uma vez que o ponto de partida do problema cosmológico é a determinação do mais relevante dos campos: $g_{\mu \nu}$, o campo gravitacional, necessitamos de um conjunto de condições adicionais, juntamente com as equações de Einstein, para determiná-lo. É nesse ponto que a cosmologia se distingue do resto da física, pois não podemos preparar um estado inicial para o universo e evoluí-lo via equações de campo. Postular as condições iniciais do universo faz parte do problema cosmológico. O princípio cosmológico é historicamente a primeira hipótese neste sentido, formulada por Einstein como hipótese simplificadora das equações de campo, e, surpreendentemente, uma ideia bem sucedida diante da evidência observacional da astronomia moderna.

Começaremos aqui, com base em conceitos, resultados e terminologias desenvolvidos no capítulo anterior, uma descrição do universo a partir do princípio cosmológico. A princípio, esta é uma premissa que não se poderia esperar explicar a partir do modelo. Mas a inflação absorve o princípio cosmológico como consequência da tentativa de resolver o principal problema do Big Bang Padrão: a impossibilidade de origem causal para o padrão de perturbações de densidade que dá origem ao universo observado. As referências 
para esse capítulo são: Weinberg (2008), Padmanabhan (1996), Mukhanov (2005), Mukhanov (2007), Peter e Uzan (2009), Kolb e Turner (1994), Lyth e Riotto (1999), Linde (2008), Brandenberger (1998), Brandenberger (1999), Brandenberger (2001), Brandenberger (2002), Brandenberger (2008), Liddle (1999)

\subsection{A cosmologia de Friedmann-Robertson-Walker e seus problemas conceituais}

O princípio cosmológico diz que existe um tempo cósmico $t$, determinado por um conjunto de observadores em queda livre com relógios sincronizados, e que observam um universo que parece o mesmo, no que diz respeito ao campo gravitacional médio, para cada observador, quando visto em qualquer direção.

Num espaço-tempo curvo, um observador $A$ que envia um sinal luminoso no instante $t_{1}$ do seu tempo próprio, sinal que é refletido num dado ponto $p$ infinitesimalmente próximo e recebido de volta por $A$ no instante $t_{2}$, determina a relação de simultaneidade entre $p$ e a posição do observador $A$ no instante $\frac{t_{1}+t_{2}}{2}$. A possibilidade de sincronização de relógios ao longo de curvas fechadas na hipersuperfície $t=0$, e consequente extensão do critério anterior para separações não infinitesimais, necessita $g_{0 \beta}=0$, o que nos leva a uma métrica da forma:

$$
d s^{2}=d t^{2}-\gamma_{i j} d x^{i} d x^{j}
$$

De fato, num sistema de coordenadas $\left(x^{0}, x^{i}\right)$ a luz viaja ao longo das geodésicas nulas conectando pontos vizinhos e tal modo que:

$$
d s^{2}=g_{00}\left(d x^{0}\right)^{2}+2 g_{0 \beta} d x^{0} d x^{\beta}+g_{i j} d x^{i} d x^{j}=0
$$

resolvendo para $d x^{0}$ :

$$
\begin{aligned}
& d x^{0(1)}=\frac{1}{g_{00}}\left\{-g_{0 i} d x^{i}-\sqrt{\left(g_{0 i} g_{0 j}-g_{0 j} g_{00}\right) d x^{i} d x^{j}}\right\} \\
& d x^{0^{(2)}}=\frac{1}{g_{00}}\left\{-g_{0 i} d x^{i}+\sqrt{\left(g_{0 i} g_{0 j}-g_{0 j} g_{00}\right) d x^{i} d x^{j}}\right\}
\end{aligned}
$$

de modo que o ponto de coordenadas $\left(x^{0}+\Delta x^{0}, x^{i}+d x^{i}\right)$ é simultâneo com $\left(x^{0}, x^{i}\right)$, onde:

$$
\Delta x^{0}=\frac{1}{2}\left(d x^{0(1)}+d x^{0(2)}\right)=-\frac{g_{0 i}}{g_{00}} d x^{i}
$$


sendo assim, para a integral de $\Delta x^{0}$ ao longo de uma curva fechada em $x^{0}=t$ ser nula, sincronização ao longo de uma curva fechada, necessitamos $g_{0 j}=0$.

Nesse sistema de coordenadas, o princípio cosmológico se traduz na existência de uma parametrização $x^{i}$ tal que a métrica seja invariante por reparametrizações da forma:

$$
x^{\prime}=R x \quad \text { e } \quad x^{\prime}=x+a
$$

onde $R$ é uma matriz de rotação arbitrária e $a$ um vetor constante arbitrário. Reciprocamente, a invariância da métrica pelas transformações 3.6 implica que $d s^{2}$ da forma diagonal 3.1. Estas transformações são obtidas transportando-se cada ponto do espaço-tempo $\mathcal{M}$ ao longo das curvas integrais de campos vetoriais gerados por combinações lineares dos vetores $\xi_{i}=\epsilon_{i j k} x^{j} \partial^{k}, \partial^{i}=\delta^{i}{ }_{j} \partial^{j}$ e $\chi_{j}=\delta^{i}{ }_{j} \partial_{i}$. Escrevemos, então, o requerimento de simetria através do conceito de Derivada de Lie 2.68:

$$
\left\{\begin{array}{c}
\mathcal{L}_{\xi_{i}} \gamma_{i j}=0 \\
\mathcal{L}_{\chi_{j}} \gamma_{i j}=0
\end{array}\right.
$$

Que geradores formam uma álgebra de Lie com respeito ao comutador, vem da identidade:

$$
\mathcal{L}_{\left[\xi_{i}, \xi_{j}\right]} X=\mathcal{L}_{\xi_{i}} \mathcal{L}_{\xi_{j}} X-\mathcal{L}_{\xi_{j}} \mathcal{L}_{\xi_{i}} X
$$

ou seja, $\mathcal{L}_{\xi_{j}} X=0$ e $\mathcal{L}_{\xi_{i}} X=0$ implicam $\mathcal{L}_{\left[\xi_{i}, \xi_{j}\right]} X=0$.

Então, o princípio cosmológico é expresso numa forma covariante por 3.7, mais a álgebra de Lie do grupo euclidiano de rotações e translações:

$$
\left[\chi_{i}, \chi_{j}\right]=0, \quad\left[\xi_{i}, \chi_{j}\right]=i \epsilon_{i j k} \chi_{k}, \quad \text { e } \quad\left[\xi_{i}, \xi_{j}\right]=i \epsilon_{i j k} \xi_{k}
$$

Que esta é uma forma covariante de expressar o princípio cosmológico vem da relação:

$$
\mathcal{L}_{X} Y=[X, Y]
$$

e da expressão da derivada de Lie em termos da derivada covariante:

$$
\left(\mathcal{L}_{X} T\right)_{e f \cdots g}^{a b \cdot c}=T_{e f \cdots g ; h}^{a b \cdot c} X^{h}-T_{e f \cdots g ; h}^{j b \cdot c} X_{; j}^{a}-(\cdots)+T_{j f \cdots g ; h}^{a b \cdot c} X_{; h}^{j}+(\cdots)
$$

As considerações acima nos levam a uma métrica da forma:

$$
d s^{2}=d t^{2}-a^{2}(t)\left(\frac{d r^{2}}{1-k r^{2}}+r^{2}\left(d \theta^{2}+\sin ^{2} \theta d \phi^{2}\right)\right)
$$


onde $a(t)$ é o fator de escala, que traduz o fato de que a única forma de evolução de uma métrica satisfazendo o princípio cosmológico é uma expansão ou contração uniforme a uma taxa maior ou igual a zero, no caso de equações de Einstein com constante cosmológica. $k$ é a curvatura da superfície de tempo constante. A expansão do universo é responsável por um importante fenômeno observacional: o redshift, oriundo da expansão do comprimento de onda de fótons emitidos no passado cósmico, um caso particular do fenômeno dinâmico de diminuição do momento das partículas em função da expansão do universo.

De fato, dada a definição do quadrimomento $p^{\mu}=m \frac{d x^{\mu}}{d s}=m u^{\mu}$, onde $s$ é o parâmetro afim, pela equação da geodésica:

$$
\frac{d u^{0}}{d s}+\Gamma_{\mu \nu}^{0} u^{\mu} u^{\nu}=0
$$

Calculando-se os coeficientes da conexão para a métrica 3.12 :

$$
\Gamma_{\mu \nu}^{0}=\frac{\dot{a}}{a} \gamma_{i j}
$$

o que implica

$$
\frac{d u^{0}}{d s}+\frac{\dot{a}}{a}|\vec{u}|^{2}=0
$$

mas uma vez que $\left(u^{0}\right)^{2}-|\vec{u}|^{2}=1$, segue que $u^{0} d u^{0}=|\vec{u}| d|\vec{u}|$, o que implica

$$
\frac{1}{u^{0}} \frac{d|\vec{u}|}{d s}+\frac{\dot{a}}{a}|\vec{u}|=0
$$

e como $u^{0} \equiv \frac{d t}{d s}$

$$
\frac{d|\vec{u}|}{d t}+\frac{\dot{a}}{a}|\vec{u}|=0
$$

que tem como solução $|\vec{u}| \propto a^{-1}$.

O redshift é denotado por $z$, tal que:

$$
1+z=\frac{a\left(t_{0}\right)}{a\left(t_{1}\right)}
$$

Uma mudança de unidades de medida de comprimento faz $k=1,0$ ou -1 , implicando em curvatura constante positiva, nula ou negativa. Que só pode existir um parâmetro caracterizando uma hipersuperfície de tempo constante satisfazendo o princípio cosmológico é devido a interpretação geométrica do tensor de Riemann como generalização do conceito de curvatura gaussiana. A curvatura gaussiana é originalmente definida para as superfícies regulares de $\mathbb{R}^{3}$ em função da aplicação normal de Gauss que mapeia cada ponto $p$ no seu 
vetor tangente normalizado, identificado com um ponto da esfera unitária $S^{3}$. A curvatura gaussiana é então o determinante da diferencial dessa aplicação que, a menos de um sinal, mede a razão entre a área, na esfera unitária, coberta pelos vetores normais da vizinhança de um ponto e a área dessa vizinhança. Este conceito, aparentemente relacionado a maneira como a superfície está mergulhada em $\mathbb{R}^{3}$, é na verdade um conceito intrínseco, como demonstrado por Gauss (Teorema Egregium), isto é, dependente apenas da métrica induzida na superfície e pode ser expresso pelo mapa exponencial:

$$
\mathcal{K}=\lim _{r \rightarrow 0} \frac{\mu\left(S_{r}(0)\right)-\mu\left(W_{r}(p)\right)}{r^{2} \mu\left(S_{r}(0)\right)}
$$

onde $\mu\left(S_{r}(0)\right)$ é a medida da área do disco $S_{r}(0)$ de raio $r$ centrado na origem do espaço tangente $T_{p} \mathcal{M}$, enquanto $\mu\left(W_{r}(p)\right)$ é a medida da área da superfície $\exp _{p}\left(S_{r}(0)\right)$.

O tensor de Riemann em $p$ é completamente determinado pelo conhecimento de todas as curvaturas gaussianas em $p$ de todas as superfícies bidimensionais obtidas por geodésicas que passam por $p$ com vetores tangentes formando um plano gerado por $u, v \in T_{p} \mathcal{M}$ no espaço tangente:

$$
\mathcal{K}=\frac{R_{\alpha \beta \mu \nu} u^{\alpha} v^{\beta} u^{\mu} v^{\nu}}{<u, u><v, v>-<u, v>^{2}}
$$

donde concluímos:

$$
\mathcal{K}=\frac{R}{n(n-1)}
$$

O tensor de Riemann tem as mesmas simetrias da métrica 3.6, o que implica necessariamente em superfícies de tempo constante de curvatura seccional constante. O que é possível se e somente para a hipersuperfície de tempo constante:

$$
R_{i j k l}=\mathcal{K} \cdot\left(g_{i k} \cdot g_{j l}-g_{i l} \cdot g_{j k}\right)
$$

A interpretação acima nos diz que o número máximo de geradores das simetrias de uma métrica de um espaço de dimensão $n$ é igual ao número de geradores das rotações de $R^{n}$ mais as translações, de modo que o espaço-tempo FRW tem hipersuperfícies maximamente simétricas, e que existe um único parâmetro $\mathcal{K}$ caracterizando-as.

Do mesmo modo, o tensor de Einstein $G_{\alpha \beta}$ 2.85 herda simetrias da métrica, que, pelo argumento já usado, implicam que é diagonal, bem como o tensor energia-momento associado. A consequência disto para o tensor energia-momento é que este é associado 
a um fluido perfeito, isto é, um fluido cujo tensor energia-momento no referencial que acompanha o movimento do fluido é diagonal:

$$
T^{\mu \nu}=\left(\begin{array}{llll}
\rho & 0 & 0 & 0 \\
0 & p & 0 & 0 \\
0 & 0 & p & 0 \\
0 & 0 & 0 & p
\end{array}\right)
$$

que, transformado para um referencial que enxerga o fluido se movendo com quadrivelocidade $U^{\mu}$ se escreve:

$$
T^{\mu \nu}=-p g^{\mu \nu}+(\rho+p) U^{\mu} U^{\nu}
$$

O tensor de Ricci é então:

$$
R_{00}=-3 \frac{\ddot{a}}{a}, \quad R_{i j}=\left(2 H^{2}+\frac{\ddot{a}}{a}+2 \frac{k}{a^{2}}\right) a^{2} \gamma_{i j}
$$

donde concluímos

$$
R=6\left(H^{2}+\frac{\ddot{a}}{a}+\frac{k}{a^{2}}\right)
$$

que nos levam a

$$
G_{00}=3\left(H^{2}+\frac{k}{a^{2}}\right), \quad G_{i j}=-\left(H^{2}+2 \frac{\ddot{a}}{a}+\frac{k}{a^{2}}\right) a^{2} \gamma_{i j}
$$

e por conseguinte às equações fundamentais da cosmologia, as equações de Friedmann:

$$
\begin{aligned}
& H^{2}=\frac{8 \pi G}{3} \rho-\frac{k}{a^{2}}+\frac{\Lambda}{3} \\
& \frac{\ddot{a}}{a}=-\frac{4 \pi G}{3}(\rho+3 p)+\frac{\Lambda}{3}
\end{aligned}
$$

onde o termo $\Lambda / 3$ vem de um possível termo adicional nas equações de Einstein da forma $\Lambda g_{\mu \nu}$ somado ao tensor de Einstein e formando o tensor simétrico conservado mais geral possível e que equivale a um fluido perfeito de equação de estado $p=-\rho$. Este tensor energia-momento é o único invariante por transformações de Lorentz, sendo, por tanto, associado a energia do vácuo que, na teoria de representação discutida mais adiante nesta tese, é definido como o estado invariante pela ação do grupo de Poincaré.

As equações 3.28 e 3.29 formam um conjunto de duas equações para determinar três incógnitas: $a(t), \rho(t)$ e $p(t)$. Necessitamos então de uma equação adicional, esta é dada 
por uma equação de estado da forma $p=p(\rho)$, a equação de um fluido isentrópico, caso particular da equação de estado dependente da densidade de entropia, $p=p(\rho, S)$. Adicionalmente, sendo um sistema de equações diferenciais de segunda ordem, necessitamos de condições iniciais, $\dot{a}\left(t_{0}\right)$ e $a\left(t_{0}\right)$, para determinar uma solução. No contexto cosmológico, alternativamente expressamos essas condições em termos dos parâmetros:

$$
H_{0} \equiv\left(\frac{\dot{a}}{a}\right)_{0} \quad \text { e } \quad \Omega=\frac{\rho}{\rho_{c r t}}
$$

onde $H_{0}$ é a constante de Hubble, escrito da forma $100 \mathrm{~h} \mathrm{~km} / \mathrm{s} / \mathrm{Mpc}$, onde $h \approx 0.7$ representa incertezas observacionais e $\Omega$ o parâmetro de densidade de matéria $\mathrm{e}$

$$
\rho_{c r t}=\frac{3}{8 \pi G}\left(\frac{\dot{a}}{a}\right)_{0}^{2}
$$

é a densidade crítica de matéria no universo da ordem de $10^{-30} \mathrm{~g} / \mathrm{cm}^{3}$, que determina o sinal da curvatura das secções de tempo constante do universo. Isso decorre da equação:

$$
\frac{k}{a^{2} H^{2}}=(\Omega-1)
$$

donde também determinarmos as condições iniciais para $a(t)$ a partir de $H_{0}$ e $\Omega$ tão $\operatorname{logo}$ $k \neq 0$.

Uma equação adicional, mas que não é independente de 3.28 e 3.29 , e que advém da conservação do tensor energia-momento $\left(T^{\alpha \beta}{ }_{; \beta}=0\right)$ é a equação da continuidade:

$$
\dot{\rho}=-3 H(\rho+p)
$$

ou ainda, escrita de outra forma:

$$
\frac{d\left(\rho a^{3}\right)}{d t}=-p \frac{d a^{3}}{d t}
$$

Observações sugerem que o universo é composto de radiação (com equação de estado $p=\frac{\rho}{3}$, para a qual reservamos o parâmetro de densidade denotado $\Omega_{\text {rad }}$ ), bárions (com equação de estado $p \cong 0$, associado ao parâmetro $\Omega_{B}$ ), matéria não bariônica (de equação de estado $p \cong 0$, associado ao parâmetro $\Omega_{N B}$ ) e possivelmente uma constante cosmológica (de equação de estado $p=-\rho$, associada ao parâmetro $\Omega_{\Lambda}$ ). Desde que não haja decaimento de uma componente na outra, estas satisfazem separadamente 3.34 para seus 
respectivos parâmetros de densidade de matéria, permitindo escrever 3.28 da seguinte forma:

$$
H^{2}+\frac{k}{a^{2}}=H_{0}^{2}\left[\Omega_{\text {rad }}\left(\frac{a_{0}}{a}\right)^{4}+\left(\Omega_{B}+\Omega_{N B}\right)\left(\frac{a_{0}}{a}\right)^{3}+\Omega_{\Lambda}\right]
$$

Com frequência, a equação acima é usada na cosmologia com a simplificação de que apenas um componente domina a expansão do universo num determinado período.

Observando a equação 3.32 , verificamos que, tão logo $\dot{a}$ seja decrescente, $\Omega(t)$ tende a se afastar de 1 ao passar do tempo, tornando este valor um ponto crítico instável das equações de evolução do universo. As observações indicam, contudo, $\Omega_{0}$ muito próximo da unidade, implicando num ajuste fino tanto maior quanto mais distante o tempo passado considerado. Este é o chamado Problema da Planura do universo.

Uma importante quantidade é a distância física percorrida por uma partícula que se move no cone de luz, o horizonte de partícula, obtida da métrica 3.12 pela condição $d s=0$ e integrado-se $d \chi=\frac{d r}{1+k r^{2}}$ ao longo da geodésica satisfazendo $d \theta=d \phi=0$ :

$$
d_{p}=a(t) \int_{0}^{a_{0}} \frac{d t}{a}=a(t) \int_{0}^{a_{0}} \frac{d a}{a \dot{a}}
$$

que impõe um limite de alcance para processos físicos conectando dois pontos. Esta distância é da ordem de grandeza do horizonte de Hubble $d_{H} \equiv H^{-1}$ em modelos satisfazendo a condição de energia forte, $\rho+3 p \geq 0$. Esta última escala é de interesse físico per si, caracterizando o tamanho do referencial localmente inercial, sendo ainda a escala relevante nas equações de perturbações cosmológicas.

Na medida em que $t \rightarrow 0$ podemos ignorar o termo $\frac{k}{a^{2}}$ em 3.28 e escrever:

$$
d_{H}^{2}=\frac{3}{8 \pi G \rho}
$$

de tal modo que

$$
\frac{d \ln d_{H}}{d t}=-\frac{1}{2} \frac{d \ln \rho}{d t}=\frac{3}{2}\left(1+\frac{p}{\rho}\right) \frac{d \ln a}{d t}
$$

uma vez que os comprimentos próprios crescem linearmente com o fator de escala $\lambda \propto a(t)$ :

$$
\frac{d \ln d_{H}}{d \ln \lambda}=\frac{3}{2}\left(1+\frac{p}{\rho}\right)
$$

donde concluímos que para $p / \rho \geq 0$ o horizonte de Hubble sempre cresce mais rápido que a separação entre observadores comóveis. Em outras palavras, desde que tomado um tempo suficientemente remoto, qualquer separação comóvel será maior que o horizonte de Hubble, 
ou ainda, dito de outro modo, cada escala cruza o horizonte uma única vez e permanece dentro do mesmo a partir de então.

Esta condição é agravada pela observação da radiação cósmica de fundo. De fato, o horizonte hoje é $H_{0}^{-1}$, considerando que num universo dominado por matéria ou radiação, este escala linearmente com o tempo cósmico $t$, e como desde a superfície de último espalhamento o universo pode ser considerado dominado por matéria, levando a $a(t) \propto t^{\frac{2}{3}}$, temos que $d_{H} \sim H_{0}^{-1}\left(1+z_{L}\right)^{-\frac{3}{2}}$, que dividida pela distância diâmetro angular ${ }^{1}$ $d_{A} \sim H_{0}^{-1}\left(1+z_{L}\right)$, para $z_{L} \sim 1100$, nos conduz a uma estimativa do ângulo compreendido pelo horizonte na última superfície de espalhamento: $1,6^{\circ}$. Contudo, a temperatura é a mesma a uma parte em $10^{5}$ ao longo de todo o céu observado, o que implica que nenhum processo físico causal poderia explicar o ajuste de temperaturas observado na CMB. Este é o problema do horizonte.

Outro problema relacionado a este advém da observação da estrutura em pequena escala do universo. A densidade de matéria não relativística dentro da esfera de raio $\lambda / 2$ é constante ao longo da evolução cósmica. Dado a densidade do universo atual, o comprimento de $1 \mathrm{Mpc}$ contêm a massa típica de uma galáxia, da ordem de $10^{11}$ massas solares $\left(M_{\odot} \approx 10^{33} \mathrm{~kg}\right)$, e este comprimento deixa o horizonte na época de domínio da radiação, mais especificamente, no redshift segundo a lei:

$$
z_{\text {enter }}(M)=\left\{\begin{aligned}
1.41 \times 10^{5}\left(\Omega h^{2}\right)^{\frac{1}{3}}\left(M / 10^{12} M_{\odot}\right)^{-\frac{1}{3}}, & M<M_{e q} \cong 3.2 \times 10^{14} M_{\odot}\left(\Omega h^{2}\right) \\
1.10 \times 10^{6}\left(\Omega h^{2}\right)^{-\frac{1}{3}}\left(M / 10^{12} M_{\odot}\right)^{-\frac{2}{3}}, & M>M_{e q} \cong 3.2 \times 10^{14} M_{\odot}\left(\Omega h^{2}\right)
\end{aligned}\right.
$$

inviabilizando uma origem causal para as perturbações de densidade que dão origem a estrutura em pequena escala do universo. Este é o problema da inomogeneidade em pequena escala.

Os comentários acima sugerem que o Big Bang padrão é incompleto enquanto modelo preditivo, e o processo faltante é o mecanismo de inflação cósmica. A inflação é o mecanismo que torna o universo observado, até primeira ordem de perturbação na métrica e tensor energia-momento, um ponto atrator, no espaço de parâmetros, de um processo físico causal. A equação 3.32 e seu comentário associado já indicam que os problemas

\footnotetext{
${ }^{1}$ Esta é definida como a distância $d_{A}$ tal que o ângulo $\theta$ subtendido por um objeto de comprimento $L$ é dado por $\theta=L / d_{A}$, fazendo-se $d s=L, d \phi=d r=0$, chegamos a $d_{A}=a(t) r$
} 
mencionados existem em um universo sempre em desaceleração. Desse modo, a inflação é uma fase de expansão acelerada para o universo, usualmente quase exponencial.

De fato, suponha uma fase de expansão (quasi)exponencial no qual o universo infla por um fator de $e^{\mathcal{N}}$, em que $\mathcal{N}$ é o número $\boldsymbol{d e}$ e-folds, e que o módulo do termo à esquerda em $3.32 \frac{|k|}{a^{2} H^{2}}$, seja da ordem da unidade no início da inflação. Este então decresce por um fator $e^{-2 \mathcal{N}}$ para o valor $\frac{|k|}{a_{I}^{2} H_{I}^{2}}$, onde o índice $I$ indica o valor no final da inflação, de modo que hoje teríamos:

$$
\frac{|k|}{a_{0}^{2} H_{0}^{2}}=e^{-2 \mathcal{N}}\left(\frac{a_{I} H_{I}}{a_{0} H_{0}}\right)^{2}
$$

De modo que para $|\Omega-1| \sim 1$ atualmente, necessitamos:

$$
e^{\mathcal{N}}>\frac{a_{I} H_{I}}{a_{0} H_{0}}
$$

Supondo que as condições não mudem muito entre o fim da inflação e início da era da radiação, estimamos $a_{I} H_{I} \approx a_{1} H_{1}$, onde o índice 1 significa valores tomados no início da era da radiação. Usando 3.35 para um universo composto de radiação e CDM:

$$
H=\frac{H_{e q}}{\sqrt{2}} \sqrt{\left(\frac{a_{e q}}{a}\right)^{3}+\left(\frac{a_{e q}}{a}\right)^{4}}
$$

onde $a_{e q}=\frac{a_{0} \Omega_{R}}{\Omega_{M}}$ e $H_{e q}=\sqrt{2 \Omega_{M}} H_{0}\left(\frac{a_{0}}{a_{e q}}\right)^{\frac{3}{2}}$ são o fator de escala e parâmetro de Hubble no momento de igualdade entre matéria e radiação. Fazendo $a=a_{1} \ll a_{e q}$ :

$$
H_{1}=\frac{H_{e q}}{\sqrt{2}}\left(\frac{a_{e q}}{a_{1}}\right)
$$

3.42 pode então ser estimado:

$$
e^{\mathcal{N}}>\left(\frac{\Omega_{M} a_{e q}}{a_{0}}\right)^{\frac{1}{4}} \sqrt{\frac{H_{I}}{H_{0}}}=\frac{\left[\rho_{1}\right]^{\frac{1}{4}}}{0.037 h e V}
$$

Tomando a escala de Planck como limite superior de energia, $\rho_{1}=\left[1.22 \times 10^{19} \mathrm{GeV}\right]^{4}$, e assumindo $h=0.7$, chegamos à estimativa $\mathcal{N} \approx 68$. Uma estimativa análoga, levando ao mesmo resultado, pode ser feito com base da condição de que o horizonte 3.36 é maior ou igual a distância diâmetro angular até a última superfície de espalhamento.

\subsection{O mecanismo de inflação dirigida por campo escalar}

\subsubsection{O rolamento lento}

Pela observação da equação 3.29, concluímos que um universo em aceleração necessita da violação da condição de energia forte $\rho+3 p \geq 0$. Devido ao sucesso Big Bang padrão 
em prever as abundâncias dos elementos primordiais, como H, He e Li, um possível período de inflação deve ocorrer numa escala de energia superior a escala da conversão neutronpróton , que marca o início da nucleossíntese, numa densidade de energia da ordem de $[1 \mathrm{MeV}]^{4}$. Dado o sucesso do modelo padrão em descrever a matéria em energias até aquelas atualmente atingidas por aceleradores, de algumas centenas de $\mathrm{GeV}$, era natural tentar mergulhar o cenário de inflação dentro do modelo padrão, baseado na ideia de campos quânticos relativísticos e simetrias de calibre. Considerando que a inflação ocorra numa escala de energia tal que a descrição da gravidade por uma teoria clássica ainda seja significativa, a matéria afeta o universo a partir do seu tensor energia-momento efetivo. No contexto de campos, somente o campo escalar com potencial é capaz de produzir um tensor energia-momento violando a condição de energia forte, enquanto mantendo compromisso com renormalizabildade. Entretanto, muito embora os potenciais abordados na literatura levem a uma teoria quântica de perturbações cosmológicas consistente, nem todos são consistentes com critérios de renormalização utilizados no cálculo de matrizes de espalhamento.

O mecanismo de inflação originalmente foi concebido dentro do contexto de teorias de grande unificação, no qual o campo escalar responsável pela quebra espontânea de simetrias dirige a inflação. Além disso, sob a hipótese de equilíbrio térmico, no contexto da teoria de transição de fases cosmológicas, hipótese esta expressa por um potencial do tipo Coleman-Weinberg. Mais ainda, a parte efetiva da inflação ocorria no estado de falso vácuo do campo escalar. Conforme discutido na introdução, todas essas hipóteses levavam a problemas e foram abandonadas nos modelos posteriores de inflação, de modo que o paradigma vigente é o de condições iniciais caóticas, possivelmente muito deslocados de seu mínimo de potencial, devido a grandes flutuações de um período Trans-Planckiano, não térmicas, e satisfazendo as chamadas condições de rolamento lento. Passaremos a discutir esse paradigma.

Conforme argumentado no segundo capítulo, o ponto de partida é uma ação compatível com o princípio da covariância geral para o campo inflaton, que dirige a inflação:

$$
S=\int d^{4} x \sqrt{-g} \mathcal{L}\left(\phi, \partial \phi, g_{\mu \nu}\right)=\int d^{4} x \sqrt{-g}\left(\partial^{\mu} \phi \partial_{\mu} \phi-V(\phi)\right)
$$

da qual deduzimos o tensor energia momento pela variação da métrica:

$$
T^{\mu \nu}=\partial^{\mu} \phi \partial^{\nu} \phi-\mathcal{L} g^{\mu \nu}
$$


e para tornar o problema da inflação tratável, fazemos a hipótese $\phi(t, x)=\phi(t)+\delta \phi(t, x)$, $\delta \phi \ll \phi$, o que nos conduz a um tensor energia-momento diagonal em primeira ordem de perturbação, mais perturbações em ordem mais elevada fora da diagonal, e por todo o raciocínio anterior a uma métrica do tipo FRW e a um fluido perfeito.

A densidade de energia e pressão desse fluido são então dados pelas equações:

$$
\begin{aligned}
& \rho_{\phi}=\frac{\dot{\phi}^{2}}{2}+V(\phi) \\
& p_{\phi}=\frac{\dot{\phi}^{2}}{2}-V(\phi)
\end{aligned}
$$

donde concluímos que se $\frac{\dot{\phi}^{2}}{2} \ll V(\phi)$, a equação de estado para esse fluido é $p \approx-\rho$. Usando a conservação do tensor energia-momento $\left(T_{; \nu}^{\mu \nu}=0\right)$ :

$$
\ddot{\phi}+3 H \dot{\phi}+V^{\prime}(\phi)=0
$$

As equações 3.28 e 3.29 com a pressão e densidade dadas por 3.48 e 3.49 .

$$
\begin{gathered}
H^{2}=\frac{8 \pi G}{3}\left(\frac{1}{2} \dot{\phi}^{2}+V+\frac{k}{a^{2}}\right) \\
\frac{\ddot{a}}{a}=\frac{8 \pi G}{3}\left(V-\dot{\phi}^{2}\right),
\end{gathered}
$$

onde, conforme o argumento sobre o problema da planura, tão logo a inflação comece, o termo de curvatura de torna desprezível, de modo que podemos ignorá-lo. Sendo assim, derivando com relação ao tempo 3.51 e usando 3.50 .

$$
\dot{H}=-4 \pi G \dot{\phi}^{2}
$$

temos ainda que, uma vez que $\dot{\phi}^{2} \ll V(\phi)$, 3.51 se simplifica para:

$$
H^{2}=\frac{8 \pi G}{3} V
$$

A equação de estado associada a $\dot{\phi}^{2} / 2 \ll V(\phi), p \approx-\rho$, quando colocado na equação 3.34 e na equação 3.28 , nos leva a uma expansão quase exponencial $a(t) \sim e^{H t}$. A condição de expansão quase exponencial é equivalente a dizer que durante um tempo característico de expansão, $\frac{1}{H}, H$ tem uma mudança fracional muito menor que a unidade, ou, equivalentemente:

$$
\left|\frac{\dot{H}}{H}\right|\left(\frac{1}{H}\right) \ll 1
$$


Do mesmo modo, é assumido que a mudança fracional em $\dot{\phi}$ durante um tempo característico de expansão $1 / H$ é muito menor que a unidade, de modo que

$$
\ddot{\phi} \ll H|\dot{\phi}|
$$

, e por consequência, a equação 3.50 se simplifica como:

$$
\dot{\phi}=-\frac{V^{\prime}(\phi)}{3 H}=-\frac{V^{\prime}(\phi)}{\sqrt{24 \pi G V(\phi)}}
$$

, pela equação 3.53, 3.55 pode ser reescrito como:

$$
\frac{\dot{H}}{H^{2}}=\frac{1}{16 \pi G}\left(\frac{V^{\prime}(\phi)}{V(\phi)}\right)^{2} \ll 1
$$

Derivando 3.57 e usando 3.57 e 3.53 .

$$
\ddot{\phi}=-\frac{V^{\prime \prime}(\phi) \dot{\phi}}{3 H}+\frac{V^{\prime}(\phi) \dot{H}}{3 H^{2}}=\frac{V^{\prime \prime}(\phi) V^{\prime}(\phi)}{9 H^{2}}-\frac{V^{\prime 3}}{48 \pi G V^{2}}
$$

que, devido a 3.56 e à simplificação de 3.50 , 3.57), implica que $\ddot{\phi} \ll V^{\prime}(\phi)$, de modo que a condição

$$
\left|\frac{V^{\prime \prime}(\phi)}{V(\phi)}\right| \ll 24 \pi G
$$

é necessária.

Os parâmetros:

$$
\epsilon=-\frac{\dot{H}}{H^{2}} \quad \text { e } \quad \delta=\frac{\ddot{\phi}}{H \dot{\phi}}
$$

são chamados de parâmetros de slow-roll, e muito da dinâmica inflacionária pode ser reescrita em termos deles:

$$
H^{2}\left(1-\frac{1}{3} \epsilon\right)=\frac{8 \pi G}{3} V, \quad \frac{\ddot{a}}{a}=H^{2}(1-\epsilon),
$$

bem como a equação de estado efetiva da inflaton como:

$$
w_{\phi}=-1+\frac{2}{3} \epsilon
$$

de modo que a condição para aceleração se reduz a:

$$
\ddot{a}>0 \Leftrightarrow w<-\frac{1}{3} \Rightarrow \epsilon<1 .
$$

Desde que $\epsilon \ll 1$ e $\delta \ll 1$, as condições de rolamento lento, as previsões da inflação são praticamente insensíveis à escolha do potencial $V(\phi)$. 
Podemos escrever o número de e-folds como:

$$
N\left(t_{i}, t_{f}\right)=\int_{t_{i}}^{t_{f}} H d t=\int_{\phi_{i}}^{\phi_{f}} H \frac{d \phi}{\dot{\phi}} \approx-\int_{\phi_{i}}^{\phi_{f}}\left(\frac{8 \pi G V(\phi)}{V^{\prime}(\phi)}\right) d \phi=-\sqrt{4 \pi G} \int_{\phi_{i}}^{\phi_{f}} \frac{d \phi}{\sqrt{\epsilon}}
$$

que nos mostra de outra perspectiva como o a resolução de problemas como o da planura e horizonte estão relacionados com as condições de rolamento lento.

As equações que governam a inflação, sendo um sistema de equações diferencias de segunda ordem, necessitam de condições iniciais $\phi\left(t_{0}\right)$ e $\dot{\phi}\left(t_{0}\right)$ para determinar uma solução. As condições de rolamento lento, contudo, tornam a inflação um atrator de um amplo espectro de condições iniciais.

Para verificar a estabilidade da solução inflacionária, tão logo a evolução de $\phi$ seja monótona, durante o rolamento lento, podemos usar $\phi$ como parâmetro ao invés de $t$ e reescrevamos a equação 3.53 como:

$$
\frac{d H}{d \phi} \equiv H_{, \phi}=-4 \pi G \dot{\phi}
$$

de tal modo que 3.51 se reescreve como $(\mathrm{k}=0)$ :

$$
H_{, \phi}^{2}-12 \pi G H^{2}(\phi)=-32 \pi^{2} G^{2} V(\phi)
$$

podemos perturbar a equação acima com respeito a solução de rolamento lento $H_{0}: H(\phi)=$ $H_{0}(\phi)+\delta H(\phi):$

$$
H_{0, \phi} \delta H_{, \phi}=12 \pi G H_{0} \delta H(\phi)
$$

que tem solução da forma:

$$
\delta H(\phi)=\delta H\left(\phi_{0}\right) \exp \left[12 \pi G \int_{\phi_{0}}^{\phi} \frac{H_{0}(\phi)}{H_{0, \phi}(\phi)} d \phi\right]
$$

que pode ser expresso através da equação 3.66 em termos do número de e-folds 3.65 .

$$
\delta H=\delta H\left(\phi_{0}\right) \exp \left[-3 N\left(t_{i}, t_{f}\right)\right]
$$

\subsubsection{Fim da inflação}

A inflação termina quando as condições de rolamento lento não são mais satisfeitas, isto é:

$$
\max (|\epsilon|,|\delta|) \sim 1
$$


onde os parâmetros $\epsilon$ e $\delta$ são dados pela Eq. 3.61.

Outra característica do mecanismo de inflação é que a mesma termina no mínimo global do potencial em $\phi=\sigma$, no qual o campo $\phi$ oscila com frequência característica $\omega^{2}=V^{\prime \prime}(\sigma)$. O universo entra então na fase oscilações coerentes, na qual $V^{\prime \prime}(\sigma) \gg H^{2}$, ou seja, o campo evolui rapidamente num tempo característico de expansão. Uma vez que durante a expansão inflacionária do universo a matéria usual de equação de estado $p=w \rho$ é diluída segundo a lei:

$$
\rho=\rho_{0}\left(\frac{a_{0}}{a}\right)^{3(1+w)}
$$

o que se deduz de 3.33 , decorre que o universo termina completamente diluído de matéria ordinária, sendo dominado por um condensado de partículas do inflaton com momento zero.

Necessitamos, por tanto, de um mecanismo que produza, ao fim da inflação, um universo dominado por radiação. Este é chamado de reaquecimento. Os detalhes do reaquecimento são um problema em aberto. Uma possível modelagem fenomenológica para o problema é assumir um termo de decaimento na equação de conservação do tensor energiamomento 3.50 ;

$$
\ddot{\phi}+3 H \dot{\phi}+\Gamma_{\phi} \dot{\phi}+V^{\prime}(\phi)=0
$$

onde este termo, $\Gamma_{\phi} \dot{\phi}$, pode advir de um termo de interação na lagrangiana do inflaton e outros campos do modelo padrão, induzindo uma taxa de decaimento $\Gamma_{\phi}$.

Multiplicando-se 3.73 por $\dot{\phi}$ e observando a expressão para densidade de energia do campo 3.49 , obtemos:

$$
\dot{\rho_{\phi}}+3 H \dot{\phi}^{2}+\Gamma \dot{\phi}^{2}=0
$$

e para tratar essa equação, retomamos a hipótese de rápida oscilação $V^{\prime \prime}(\sigma) \gg H^{2}$ e substituímos $\dot{\phi}$ por sua média ao longo de um ciclo de oscilação, em que

$$
\langle V\rangle=\left\langle\frac{\dot{\phi}^{2}}{2}\right\rangle=\frac{\rho_{\phi}}{2},
$$

que nos leva a

$$
\left\langle\dot{\phi}^{2}\right\rangle_{\text {ciclo }}=\rho_{\phi}
$$

e consequentemente a

$$
\dot{\rho_{\phi}}+3 H \rho_{\phi}+\Gamma \rho_{\phi}=0
$$


A resolução dessa equação necessita o conhecimento de $H$, mas podemos usar o conhecimento da equação de estado do inflaton:

$$
\left\langle p_{\phi}\right\rangle=\left\langle\frac{\dot{\phi}^{2}}{2}-V(\phi)\right\rangle=0
$$

o que nos leva, pela equação $3.35 \operatorname{com} \Omega_{\text {rad }}=\Omega_{\Lambda}=k=0$, a $a \propto t^{\frac{2}{3}}$ e $H=\frac{2}{3 t}$, e por conseguinte:

$$
\rho(t)=\rho_{0} t^{-2} e^{-\Gamma_{\phi}\left(t-t_{0}\right)}=M^{4}\left(\frac{a}{a_{o s c}}\right)^{-3} e^{-\Gamma_{\phi}\left(t-t_{0}\right)} .
$$

Essa aproximação é justificável até um tempo $t \sim \Gamma_{\phi}^{-1}$, após o que, a componente na qual decai, a radiação ordinária, passa a dominar a dinâmica. $a_{o s c}$ é o fator de escala do início da fase de oscilações.

De fato, o conjunto completo de equações necessita adicionalmente de:

$$
\begin{gathered}
\dot{\rho_{R}}+4 H \rho_{R}=\Gamma_{\phi} \rho_{\phi} \\
H^{2}=8 \pi G\left(\rho_{\phi}+\rho_{R}\right) / 3
\end{gathered}
$$

onde 3.80 vem de um termo de criação ao lado direito da equação de conservação do tensor energia-momento da radiação.

Podemos então escrever uma solução aproximada para $\rho_{R}$ em 3.80 , no regime $t<\Gamma_{\phi}^{-1}$, no qual o universo é dominado por matéria, $H=\frac{2}{3 t}$, e o termo de decaimento do inflaton, $\Gamma_{\phi} \phi$, pode ser desprezado em 3.73 juntamente com o termo de potencial ao fim a inflação, implicando $\rho_{\phi} \propto a^{-3} \propto\left(t / t_{o s c}\right)^{-2}$, onde $t_{o s c}$, início da fase de oscilação, pode ser tomado como $H^{-1}=\left(\frac{8 \pi G}{3} M^{4}\right)^{-1}=\left(\frac{8 \pi}{3 m_{P l}^{2}} M^{4}\right)^{-1}$, numa aproximação em que $\Gamma_{\phi}^{-1}$ é muito maior que o momento de início da inflação. Isso nos leva a:

$$
\rho_{R} \approx \frac{m_{P l}^{2} \Gamma_{\phi}}{10 \pi t}\left[1-\left(\frac{t}{t_{o s c}}\right)^{-\frac{5}{3}}\right] \approx \frac{\left(\frac{6}{\pi}\right)^{\frac{1}{2}}}{10} m_{P l} \Gamma_{\phi} M^{2}\left(\frac{a}{a_{o s c}}\right)^{-\frac{3}{2}}\left[1-\left(\frac{a}{a_{o s c}}\right)^{-\frac{5}{2}}\right]
$$

Podemos então calcular a temperatura do início da era da radiação, ou Temperatura de reaquecimento sabendo sua densidade de energia $\rho_{R}=\frac{\pi^{2}}{30} g_{*} T^{4}$, onde $g_{*}$ é o número total efetivo de graus de liberdade das partículas relativísticas que compõem o universo (Mais sobre a termodinâmica da radiação será dito quando discutirmos o modelo de inflação não-comutativa):

$$
T_{R H} \equiv T\left(t=\Gamma_{\phi}^{-1}\right) \sim 0.55 g_{*}^{-\frac{1}{4}}\left(m_{P l} \Gamma_{\phi}\right)^{\frac{1}{2}}
$$


Com base nessas informações, podemos fazer uma nova estimativa do número de e-folds sem a hipótese anterior de que as condições do universo não mudam muito entre o fim do rolamento lento e início da era da radiação. Consideremos que o universo se expande por um fator $\exp (\mathcal{N})$ durante o rolamento lento, e durante o reaquecimento por um fator $\left(M^{4} / T_{R H}^{4}\right)^{\frac{1}{3}}$, devido a dominação por matéria. Considerando que o universo observado começa dentro do horizonte na época do início da inflação, determinado pela escala de energia da inflação, $H^{-1} \sim \frac{m_{P l}}{M^{2}}$, a entropia final $\left(\propto T^{3}\right)$ será:

$$
S \sim \exp 3 \mathcal{N}\left(\frac{M^{4}}{T^{4}}\right) H^{-3} T_{R H}^{3} \sim \exp 3 \mathcal{N} \frac{m_{P l}^{3}}{M^{2} T_{R H}}
$$

e fazendo essa entropia ser maior do que a entropia observada dentro do horizonte atual, da ordem de $10^{88}$ :

$$
\mathcal{N} \geq 53+\frac{2}{3} \ln \left(\frac{M}{10^{14} \mathrm{GeV}}\right)+\frac{1}{3} \ln \left(\frac{T_{R H}}{10^{10} \mathrm{GeV}}\right)
$$

Considerando como limite inferior para a escala de energia da inflação não o limite da escala de energia da nucleossíntese (da ordem da escala conversão neutron-próton), 1Mev, mas sim a escala de energia dos aceleradores, da ordem de $G e V$, bem como a escala de Planck como limite superior, tanto para $M$ quanto para $T_{R H}$, temos que $\mathcal{N}$ varia entre 24 e 68. 
Capítulo 4

\section{Teoria de Perturbações cosmológicas}

\subsection{Introdução}

O princípio cosmológico advoga um universo que parece o mesmo quando visto de qualquer lugar e em qualquer direção num mesmo tempo. Esta foi uma ideia bastante frisada no capítulo anterior. Contudo, assumindo a validade do princípio, a simples inspeção do ambiente a nossa volta nos leva a conclusão da existência de uma escala de comprimento abaixo da qual o universo é altamente inomogêneo e anisotrópico. Esta inomogeneidade pode ser caracterizada de muitas maneiras. Poderíamos, num dado instante cósmico, observar a quantidade de massa no interior de uma esfera de raio $\lambda$ e centro $p$ e compará-la com a densidade média do universo calculando-se a diferença percentual entre aquela e a que se esperaria num universo homogêneo. Num universo homogêneo e isotrópico, esperamos uma média em $p$ dessa quantidade igual a zero, mas o desvio padrão, raiz quadrada da média dos quadrados dessa quantidade, $\left\langle\left(\frac{\delta M}{M}\right)_{\lambda}^{2}\right\rangle$, é um importante caracterizador das inomogeneidades do universo nas escalas de tamanho $\lambda$. Ao longo do intervalo de escalas que compreende a estrutura não linear observada hoje, variando de $1 \mathrm{Mpc}$ a $10 \mathrm{Mpc}$, o universo é compatível com um padrão de inomogeneidades que, no instante $t$, em que $\lambda=H^{-1}$ satisfaz $\left\langle\left(\frac{\delta M}{M}\right)_{\lambda}^{2}\right\rangle=$ const. Este fato não tem explicação dentro do modelo em desaceleração, ou seja, satisfazendo as condições de energia forte, em que uma escala adentra o horizonte após ter estado fora ao longo de toda história cósmica anterior.

Este padrão de flutuações se chama Harrison-Zel'dovich e, como veremos, é uma previsão genérica da inflação, a menos de característicos desvios em pequenas escalas associados ao fim da inflação. Isso se deve ao modelo de inflação dirigido por campo escalar ser descrito por uma expansão quase exponencial, o que está associado ao chamado espaço 
de Sitter. Este é maximamente simétrico em quatro dimensões, no sentido anteriormente descrito em 3 para modelos FRW no capítulo anterior.

De fato, o espaço de Sitter pode ser obtido da métrica induzida no hiperbolóide

$$
\left(x^{0}\right)^{2}-\left(x^{1}\right)^{2}-\left(x^{2}\right)^{2}-\left(x^{3}\right)^{2}-\left(x^{4}\right)^{2}=-H_{\Lambda}^{-2},
$$

uma superfície invariante pelo grupo de Lorentz homogêneo em cinco dimensões, mergulhado no espaço Minkowski de mesma dimensionalidade (é de fato uma órbita do grupo, conforme a discussão sobre simetrias no capítulo posterior). Este possui dez geradores, o número máximo de simetrias que um espaço métrico de quatro dimensões pode ter, conforme o argumento do capítulo anterior, sendo quatro destes geradores boosts e seis, rotações. Sendo assim, um mecanismo de geração de perturbações, num fundo de quase simetria de translação temporal, produz níveis de distorção quase iguais em cada escala ao longo da saída das mesmas, marco do instante a partir do qual a física causal não mais pode afetar sua forma.

Descreveremos este mecanismo aqui, com base nas mesmas referências do capítulo anterior. Adicionalmente, Gotay (1999) e Zainuddin et al. (2007), para questões técnicas envolvendo a quantização na subseção 4.4.2.

\subsection{A descrição estatística da matéria}

Existem essencialmente três níveis de descrição clássica da matéria no universo, cada um tendo seu domínio de aplicabilidade e significando uma perda de informação em relação a anterior:

- Podemos considerar a trajetória de cada partícula, $x(t)$, ao longo da história cósmica;

- Podemos considerar a função de distribuição no espaço de fases, $f(x, p, t)$;

- Podemos considerar a densidade (ou contraste de densidade) de matéria em função do tempo e posição, $\rho(t, x)$, bem como a velocidade média das partículas $v(t, x)$;

A descrição por trajetórias é objeto de grandes simulações numéricas de elevado custo computacional e nem sempre fácil interpretação, enquanto que a descrição pela densidade é viável quando efeitos de dispersão de velocidade entre as partículas não são relevantes ao 
ponto de afetar a evolução do contraste de densidade, quando então a descrição por função de distribuição é preferível. Sob condições de equilíbrio termodinâmico, por exemplo, os valores médios de velocidade são sobrepujantemente mais prováveis que qualquer outro valor, viabilizando uma descrição pela densidade, enquanto que neutrinos após o desacoplamento $]^{1}$ são melhor descritos pela distribuição no espaço de fases e um equação do tipo Boltzmann.

Não podemos, contudo, esperar que a teoria preveja a forma exata de $\rho(x, t)$ e/ou $f(x, p, t)$, mas sim suas propriedades estatísticas. Relacionado a esse problema, o princípio cosmológico ganha uma versão aplicável a perturbações: homogeneidade e isotropia estatísticas. O que significa que, dividindo-se o universo em regiões retangulares $R_{1}, R_{2} \ldots R_{N}$ de mesmo tamanho, a flutuação de densidade $\delta \equiv \frac{\delta \rho}{\rho} \equiv \frac{\rho-\bar{\rho}}{\bar{\rho}}$, ou equivalentemente a densidade $\rho(x, t)$, em cada uma delas é uma realização da mesma variável aleatória, independente da posição e orientação da região. Dito de outro modo, o funcional de probabilidade, que condifica a informação sobre o campo aleatório $\rho(x, t)$, é invariante por translação e rotação, ou seja, depende apenas de $\rho(x, t)$ e do tempo:

$$
\mathcal{P}=\mathcal{P}[\rho(x, t) ; t]
$$

Dado um funcional de probabilidades $\mathcal{P}$, podemos calcular a probabilidade de $\rho$ pertencer a qualquer conjunto mensurável $\mu \mathrm{I}^{2}$ de possíveis padrões de flutuação, segundo a integral associada.

Um maneira alternativa de caracterizar a estatística é usar um conjunto de quantidades que possua a mesma de informação que o funcional de probabilidade. Dentre estas quantidades, é especialmente relevante a função de correlação de dois pontos da função $f(x)$ codificando a informação sobre a matéria:

$$
\xi_{f}(x)=\langle f(x+y) f(y)\rangle
$$

\footnotetext{
${ }^{1}$ Quando a taxa de interação da matéria (por espalhamento, por exemplo) não é suficiente para manter o equilíbrio termodinâmico. Uma caracterização dessa situação é obtida comparando-se o tempo médio entre $t_{s c}$, caracterizado por uma secção de choque $\sigma$, uma densidade $n$ e uma velocidade relativa $v, t_{s c}=\frac{1}{\sigma n v}$ com o tempo de Hubble $H^{-1}: t_{s c} \gg H^{-1}$ implica em desacoplamento

${ }^{2}$ Observe que, segundo o sentido definido na teoria da medida e integração, só podemos definir a integração do funcional de probabilidades após a definição dos conjuntos mensuráveis e suas respectivas medidas no espaço das funções $\rho(x, t)$, a $\sigma$-algebra. Esta não está a priori definida
} 
onde $<>$ denota a média em $y$ num grande volume $V$ que, sob a hipótese de homogeneidade estatística e em um universo idealizado infinito, é equivalente a uma média no ensemble. Ainda sob essas mesmas hipóteses, conclui-se que $\xi_{f}(x)$ depende apenas do módulo de $x$.

A função de dois pontos, por um lado é uma importante caracterização da distribuição estatística que governa $f(x)$, pois é um dos momentos da distribuição e é possível reconstruir a mesma a partir dos seus momentos. Por outro lado, caracteriza o excesso de probabilidade de se encontrar uma região sobredensa ou subdensa a uma determinada distância de um ponto respectivamente sobredenso ou subdenso.

Com efeito, podemos integrar, sob hipótese de homogeneidade estatística, $\xi_{\delta}(x)$ :

$$
\int d^{3} x \xi_{\delta}(x)=\int d^{3} x\langle\delta(x+y) \delta(y)\rangle=\left\langle\int d^{3} x \delta(x+y) \delta(y)\right\rangle=0
$$

que sob isotropia se escreve como:

$$
\int d x 4 \pi x^{2} \xi_{\delta}(x)=0
$$

que implica na existência e um zero em $|x|=L$, que caracteriza o tamanho típico de regiões sobredensas ou vazios.

Outra razão para sua importância é o padrão gaussiano de flutuações de densidade que, como veremos, é uma previsão genérica dos modelos de inflação e o padrão sugerido pelas observações atuais, que é completamente caracterizado pela função de dois pontos.

De fato, indo para o espaço de Fourier $\delta_{k}=\int d^{3} k \delta e^{i k x}$, no regime linear, em que as equações diferenciais para $\delta_{k}$ se desacoplam, podemos considerar a fatoração do funcional $\mathcal{P}$ em distribuições independentes para cada componente $\delta_{k}$. Assim sendo, a distribuição Gaussiana é definida atribuindo-se para cada $\delta_{k}$ a distribuição (assumimos aqui a função $\rho(x, t)$ como definida numa caixa finita, por simplicidade de notação):

$$
p\left(a_{k}, b_{k}\right)=\frac{1}{\sqrt{2 \pi \sigma(t)_{k}^{2}}} e^{-\frac{a_{k}^{2}+b_{k}^{2}}{2 \sigma(t)_{k}^{2}}}=\frac{1}{\sqrt{2 \pi \sigma(t)_{k}^{2}}} e^{-\frac{\left|\delta_{k}\right|^{2}}{2 \sigma(t)_{k}^{2}}}
$$

que nos leva a um funcional da forma:

$$
\mathcal{P}[\delta(x, t) ; t]=N \exp \left[\int d x d y \delta(x) F(x-y) \delta(y)\right]
$$

onde

$$
F(x-y)=\sum_{k} \frac{e^{i k(x-y)}}{\sigma(t)_{k}^{2}}
$$


devido a

$$
\left|\delta_{k}\right|^{2}=\int d^{3} x d^{3} y \delta(x) \delta(y) e^{i k(x-y)}
$$

Um funcional análogo a 4.7 ocorre na função geradora das funções de Green das teorias livres (sem interação entre as partículas), cujas derivadas funcionais com relação a função fonte nos dão a função de correlação para os campos (ordenada no tempo), recebendo também o nome de funcional Gaussiano 3 . Conforme veremos, o padrão Gaussiano surge devido ao fato de que as perturbações cosmológicas, na aproximação de slow roll, são campos livres e, por conseguinte, com função geradora de correlações Gaussiana.

Assumindo então um padrão Gaussiano de flutuações, reescrevemos a função de dois pontos como:

$$
\xi_{\delta}(x)=<\delta(x) \delta(x+y)>=\frac{1}{V^{2}} \sum_{q k}<\delta_{k} \delta_{q}^{*}>e^{i k x-i q y(x-y)}=\frac{1}{V^{2}} \sum_{k} \sigma_{k}^{2} e^{i k y}
$$

onde usamos

$$
<\delta_{k} \delta_{q}>=\delta_{k p} \sigma_{k}^{2}
$$

sendo $\delta_{k q}$ a delta de Kronecker, resultado esse derivado da distribuição 4.6 .

No espaço de Fourier, tomado o limite de caixa infinita, o padrão Gaussiano é caracterizado como:

$$
<\delta(k) \delta(q)>=\sigma_{k}^{2} \delta(k+q)
$$

sendo

$$
\delta(x)=\frac{1}{\sqrt{V}} \int \delta_{k} e^{i k x} \frac{d^{3} k}{(2 \pi)^{\frac{3}{2}}}=\int \delta(k) e^{i k x} \frac{d^{3} k}{(2 \pi)^{\frac{3}{2}}}
$$

e a função de correlação tem então uma simples caracterização:

$$
\xi_{\delta}(x)=\int \frac{d^{3} k}{(2 \pi)^{3}} P_{\delta}(k) e^{i k x}, \quad P_{\delta}(k)=\left|\sigma_{k}\right|^{2} V^{-1}
$$

em que $P_{\delta}(k)$ é o chamado power spectrum de $f(x)$, o limite de grandes volumes da expressão $\sigma_{k}^{2} / V$, transformada de Fourier do contraste de densidade sob a hipótese de homogeneidade estatística. A isotropia nos leva então a:

$$
\xi_{f}(x-y)=\frac{1}{V} \int \frac{\sigma_{k}^{2} k^{3}}{2 \pi^{2}} \frac{\sin (k r)}{k r} \frac{d k}{k}
$$

\footnotetext{
${ }^{3} Z_{0}[J]=N \exp \left[-\frac{1}{2} \int d x d y J(x) \Delta_{F}(x-y) J(y)\right]$, onde $\Delta_{F}(x-y)$ é propagador de Feymann, valor esperado no vácuo do produto ordenado no tempo de dois operadores de campo, $\left\langle 0\left|T \phi\left(x_{1}\right) \phi\left(x_{2}\right)\right| 0\right\rangle$, e a função de correlação é dada por $G^{(N)}\left(x_{1}, \ldots, x_{N}\right)=\left\langle 0\left|T \phi\left(x_{1}\right) \cdots \phi\left(x_{N}\right)\right| 0\right\rangle=(-i)^{N} \frac{\delta^{N} Z[J]}{\delta J\left(x_{1}\right) \cdots \delta J\left(x_{N}\right)}$
} 
Que a função de dois pontos é o único momento relevante é consequência do teorema $\left.\boldsymbol{d e} \boldsymbol{W} \boldsymbol{i} \boldsymbol{c k}\right|^{4}$ que diz que a função de $N$-pontos se fatora em termos da função de dois pontos para uma estatística gaussiana:

$$
\left\langle\delta\left(x_{1}\right) \delta\left(x_{2}\right) \cdots \delta\left(x_{N}\right)\right\rangle=\sum_{P} \prod_{(i, j) \in P}<\delta\left(x_{i}\right) \delta\left(x_{j}\right)>
$$

onde $P$ é uma partição dos $N$ elementos em pares $(i, j)$ disjuntos, sendo $i \neq j$. Mais que isso: a propriedade 4.16 é suficiente para caracterizar completamente o padrão Gaussiano.

Uma generalização de 4.3 é, ao invés de considerar o valor de $f$ em $x$, considerar uma média de $f$ em torno de $x$ com a função filtro $W(y)$ :

$$
f_{W}(x)=\int f(x+y) W(y) d^{3} y
$$

podemos então considerar a média:

$$
\left\langle f_{W}^{2}\right\rangle=\int \frac{d^{3} k}{(2 \pi)^{3}} P(k)|W(k)|^{2}
$$

Um caso particular é a flutuação média quadrática da massa dentro da esfera de raio $R$, $\left\langle\left(\frac{\delta M}{M}\right)_{R}^{2}\right\rangle$, que é dada por 4.17 com $f=\frac{\delta \rho}{\rho}$ e $W(y)=1$ para $|y| \leq R$, zero em caso contrário:

$$
\sigma^{2}(R)=\left\langle\left(\frac{\delta M}{M}\right)_{R}^{2}\right\rangle=\int_{0}^{\infty} \frac{d k}{k} \frac{k^{3} P(k)}{2 \pi^{2}}\left[\frac{3 \sin k R}{(k R)^{3}}-\frac{3 \cos k R}{(k R)^{2}}\right]^{2}
$$

em que $\delta_{f}^{2} \equiv \frac{k^{3} P(k)}{2 \pi^{2}}$ é chamada de variância adimensional. Expressão essa que, com muita frequência, é substituída pela aproximação:

$$
\left\langle\left(\frac{\delta M}{M}\right)_{R}^{2}\right\rangle \sim \delta_{f}^{2}\left(k \sim \frac{2 \pi}{\lambda}\right)
$$

\subsection{O problema de calibre das perturbações}

Na seção anterior, descrevemos um padrão estatístico de flutuações de densidade, o gaussiano e invariante de escala. Começaremos aqui a descrever o ferramental que leva

\footnotetext{
${ }^{4}$ que tem uma versão em teoria de campos e em processos estocásticos de um modo geral, sendo os dois relacionados pela fórmula de Feymann-Kac que expressa a integral de trajetória em termos de um processo estocástico.
} 
a previsão desse padrão a partir da inflação. A evolução das componentes que descrevem as perturbações da métrica não são completamente determinadas pelas equações dinâmicas, restando uma liberdade que pode ser excluída pela escolha de um novo conjunto de variáveis, a escolha deste conjunto é o que se entende por uma escolha de calibre.

Durante a inflação, o horizonte de Hubble, $H^{-1}$, permanece constante enquanto a separação entre observadores comoveis, ou comprimento de onda das perturbações, aumenta exponencialmente. Não é a princípio óbvio que a forma do espectro não se altere ao longo da evolução cósmica para modos de comprimento maior que $H^{-1}$, uma vez que argumentamos que a inflação ocorre durante uma violação da condição de energia forte e, apenas enquanto válida assegura o horizonte de partícula da ordem do horizonte de Hubble. É uma conclusão a posteriori, que a escala relevante na evolução de perturbações é a escala de Hubble. A antecipação desta, contudo, nos leva a conclusão de que o problema de geração de perturbações envolve modos de comprimento muito maior que o tamanho característico do referencial localmente inercial no qual se esperaria que uma descrição Newtoniana das perturbações fosse aplicável, exigindo, por tanto, uma descrição relativística.

As equações de Einstein são em número de dezesseis em variáveis reais (igualdade entre números reais), considerando-se a simetria do tensor de Einstein, caímos para dez equações para determinação de dez incógnitas da métrica. Considerando que a identidade de Bianchi, que são quatro equações, é automaticamente satisfeita pelo tensor de Einstein, representando, por tanto, um vínculo, quatro dessas equações são dependentes das demais. Terminamos com quatro graus de liberdade nas variáveis de campo que não são determinadas pelas equações de Einstein. Estes estão associados à invariância por difeomorfismo da teoria, manifestando-se na forma de quatro geradores infinitesimais para o difeomorfismo:

$$
\bar{x}^{\mu}=x^{\mu}+\varepsilon \xi^{\mu}(x)
$$

O princípio cosmológico privilegia um sistema de coordenadas: aquele em que as simetrias assumem a forma do grupo euclidiano de translações e rotações. Quando, porém, consideramos um universo com perturbações de densidade, o sistema de coordenadas é privilegiado apenas em ordem zero de perturbação, estando a priori indefinido em ordens mais elevadas:

$$
d s^{2}=\left[{ }^{(0)} g_{\alpha \beta}+\delta g_{\alpha \beta}\right] d x^{\alpha} d x^{\beta}
$$


em que

$$
{ }^{(0)} g_{\alpha \beta} d x^{\alpha} d x^{\beta}=a^{2}(\eta)\left(d \eta^{2}-\delta_{i j} d x^{i} d x^{j}\right)
$$

e $d \eta \equiv d t / a$ é a diferencial do tempo conforme, $\eta$.

Necessitamos, então, excluir quatro graus de liberdade das perturbações, $\delta g_{\alpha \beta}$, associados a artefatos, perturbações fictícias. Para tanto, decompomos as perturbações em componentes cujas regras de transformação podem ser consideradas independentemente, o que resulta ser mais simples que considerar a regra de transformação tensorial.

Emprega-se então, nas perturbações, a decomposição $\boldsymbol{S} \boldsymbol{T} \boldsymbol{V}$, ou seja, escalar, tensorial e vetorial:

$$
\delta g_{00}=2 a^{2} \phi
$$

onde $\phi$ é um 3-escalar, isto é, transforma-se como escalar pelo grupo Euclidiano de transformações;

$$
\delta g_{0 i}=a^{2}\left(B_{, i}+S_{i}\right)
$$

sendo $B$ um escalar, $B_{, i} \equiv \frac{\partial B}{\partial x^{i}}$ e $S_{i}$ um 3-vetor, também com respeito ao grupo Euclidiano. $S^{i}$ obedece ainda ao vínculo de divergência nula, $S_{, i}^{i}=0$, sendo a delta de Kronecker usada para levantar e abaixar índices.

$$
\delta g_{i j}=a^{2}\left(2 \psi \delta_{i j}+2 E_{, i j}+F_{i, j}+F_{j, i}+h_{i j}\right),
$$

onde $\psi$ e $E$ são escalares, $F_{i}$ é um vetor de divergência nula, $F^{i}{ }_{i}=0$, e $h_{i j}$ é simétrico e tem divergência e traço nulos:

$$
h_{i}^{i}=0, \quad h_{j, i}^{i}=0
$$

Esta decomposição é única sob a hipótese de rápido decrescimento das perturbações $\delta g_{\alpha \beta}$ no infinito. De fato, 4.25 pode ser reescrita como:

$$
\left\{\begin{array}{c}
\nabla^{2} B=a^{-2} \partial^{i} \delta g_{0 i} \\
S_{i}=a^{-2} \delta g_{0 i}-B_{, i}
\end{array}\right.
$$

enquanto 4.26 nos leva a:

$$
\left\{\begin{array}{c}
\nabla^{2} \psi+\nabla^{4} E=\frac{1}{2} a^{-2} \partial_{i} \partial_{i} \delta g_{i i} \\
\nabla^{2} E=\frac{1}{2} a^{-2} \delta g_{i i} \\
\nabla^{2} F_{i}=a^{-2} \partial_{j} \delta g_{j i}-\partial_{i}\left(2 \psi+2 \nabla^{2} E\right) \\
h_{i j}=a^{-2} \delta g_{i j}-\left(2 \psi \delta_{i j}+2 E_{, i j}+F_{i, j}+F_{j, i}\right)
\end{array}\right.
$$

\footnotetext{
5 índices gregos variam de 0 a 3 , enquanto índices latinos de 1 a 3 , representando coordenadas espaciais
} 
A hipótese de rápido decrescimento (num sentido mais abrangente do que o empregado na teoria de distribuições, por exemplo) pode então ser dada em termos de cada uma das variáveis que são soluções de equações do tipo Poisson, denotadas coletivamente por $\phi$, de modo a assegurar unicidade do problema de contorno (problema de Dirichlet ou Von Neumann $\left.]^{6}\right]^{7}$

As componentes escalares, dentre estas, são as mais importantes, pois são estas que se acoplam às perturbações de densidade via equações de Einstein e, por conseguinte, são as componentes associadas à instabilidade gravitacional. As componentes vetoriais estão associadas à componente rotacional do campo de velocidades, mas, diferentemente das perturbações escalares, decaem ao longo da evolução cósmica. As componentes tensoriais descrevem as ondas gravitacionais, presentes apenas em teorias métricas, não participando contudo, na aproximação linear, da instabilidade gravitacional.

Sob a transformação 4.21, podemos assumir, em primeira ordem, que nenhum tensor é afetado, a métrica de background entre eles. Mas as perturbações de primeira ordem em $\epsilon, \delta T$, de qualquer tensor, $T$, transformam-se segundo lema de Stewart-Walker, que é um consequência direta da definição 2.68 .

$$
\delta \bar{T}=\delta T-\epsilon \mathcal{L}_{\xi} T
$$

Utilizando 2.69, escrevendo-se $\xi=\left(\xi^{0}, \xi^{i}\right)$ e decompondo-se $\xi^{i}$ do mesmo modo que 4.25

$$
\xi_{\perp}^{i}+\varsigma^{, i}
$$

\footnotetext{
${ }^{6}$ Quando a valor de $\phi$, solução de uma equação de Poisson, é especificado na fronteira de um aberto, este é o problema de contorno de Dirichlet. Quando alternativamente especificamos a derivada na direção normal a cada ponto da fronteira, este é o problema de Von Neumann

${ }^{7}$ Segundo a primeira identidade de Green, $\int_{V} d^{3} x \phi \nabla^{2} \phi=-\int_{V} d^{3} x(\nabla \phi, \nabla \phi)+\int_{\partial V} d s \phi \frac{\partial \phi}{\partial n}$, em que $(\nabla \phi, \nabla \phi)$ denota o produto interno dos gradientes e $n$ o vetor normal da fronteira de $V$, denotada $\partial V$. Sendo assim, dado duas soluções da equação de Poison, $\phi_{1}$ e $\phi_{2}$, que decaiam suficientemente rápido, de tal modo que: $\int_{\partial V} d s \phi_{1,2} \frac{\partial \phi_{1,2}}{\partial n} \rightarrow 0$, a diferença entre elas, $\phi_{1-2}$, é uma solução da equação de La'Place, tal que: $\int_{V} d^{3} x\left(\nabla \phi_{1-2}, \nabla \phi_{1-2}\right) \rightarrow 0$, implicando em $\phi_{1-2}=0$. Logo, não existem duas soluções diferentes da equação de Poisson que pertençam ao subespaço satisfazendo $\int_{\partial V} d s \phi_{1,2} \frac{\partial \phi_{1,2}}{\partial n} \rightarrow 0$, o que garante a unicidade da decomposição STV.
} 
$\operatorname{com} \xi_{\perp, i}^{i}=0$, decorre que:

$$
\begin{aligned}
\delta \bar{g}_{00} & =\delta g_{00}-2 a\left(a \xi^{0}\right)^{\prime} \\
\delta \bar{g}_{0 i} & =\delta g_{0 i}+a^{2}\left[\xi_{\perp i}^{\prime}+\left(\varsigma^{\prime}-\xi^{0}\right)_{, i}\right] \\
\delta \bar{g}_{i j} & =\delta g_{i j}+a^{2}\left[2 \frac{a^{\prime}}{a} \delta_{i j} \xi^{0}+2 \varsigma_{, i j}+\left(\xi_{\perp_{i, j}}+\xi_{\perp_{i, j}}\right)\right]
\end{aligned}
$$

em que ${ }^{\prime}$ denota a derivada com respeito ao tempo conforme.

As componentes escalares das perturbações, em termos das quais a métrica de escreve como

$$
d s^{2}=a^{2}\left[(1+\phi) d \eta^{2}+2 B_{, i} d x^{i} d \eta-\left((1-2 \psi) \delta_{i j}-2 E_{, i j}\right) d x^{i} d x^{j}\right],
$$

transformam-se então como:

$$
\begin{aligned}
& \phi \rightarrow \bar{\phi}=\phi-\frac{1}{a}\left(a \xi^{0}\right)^{\prime}, \quad \text { e } \quad B \rightarrow \bar{B}=B+\varsigma^{\prime}-\xi^{0}, \\
& \psi \rightarrow \bar{\psi}=\psi+\frac{a^{\prime}}{a} \xi^{0}, \quad \text { e } \quad E \rightarrow \bar{E}=E+\varsigma .
\end{aligned}
$$

Uma consequência de 4.32 e da unicidade da decomposição STV.

Para perturbações vetoriais, em termos das quais a métrica se escreve como

$$
d s^{2}=a^{2}\left[d \eta^{2}+2 S_{i} d x^{i}-\left(\delta_{i j}-F_{i, j}-F_{j, i}\right) d x^{i} d x^{j}\right]
$$

temos que

$$
S_{i} \rightarrow \bar{S}_{i}=S_{i}+\xi_{\perp i}^{\prime} \quad \text { e } \quad F_{i} \rightarrow \bar{F}_{i}+\xi_{\perp i},
$$

enquanto que as perturbações tensoriais, em termos das quais a métrica se escreve como

$$
d s^{2}=a^{2}\left[d \eta^{2}-\left(\delta_{i j}-h_{i j}\right) d x^{i} d x^{j}\right]
$$

são invariantes.

Podemos então remover os graus de liberdade associados ao calibre pela construção de um conjunto de seis quantidades independentes e invariantes pela ação de difeomorfismos. Duas dessas são obtidas de combinações de componentes escalares:

$$
\Phi \equiv \phi-\frac{1}{a}\left[a\left(B-E^{\prime}\right)\right]^{\prime}, \quad \Psi \equiv \psi+\frac{a^{\prime}}{a}\left(B-E^{\prime}\right)
$$

recebendo o nome de potenciais de Bradeen.

Outros dois componentes, considerando os vínculos associados a $S_{i}$ e $F_{i}$, são obtidos pela construção de

$$
\bar{V}_{i}=S_{i}-F_{i}^{\prime}
$$


enquanto que os dois componentes restantes são associados aos modos de vibração das ondas gravitacionais: seis componentes de $h_{i j}$, menos quatro vínculos.

Alternativamente, podemos remover graus de liberdade de calibre pela imposição de condições de calibre, que restringem a escolha do sistema de coordenadas, podendo ou não determiná-lo unicamente. Uma das mais importantes escolhas é aquela em que $\phi=\Psi$ e $\Phi=\phi$, determinado pelas condições $E=B=0$, conhecido como calibre Newtoniano ou longitudinal.

Podemos então perturbar as equações de Einstein, obtendo desse modo as equações para evolução das perturbações. A perturbação de primeira ordem do tensor de Einstein é então igualada à perturbação do tensor energia-momento:

$$
\delta G_{\beta}^{\alpha}=\delta T_{\beta}^{\alpha}
$$

Estas perturbações não são gauge invariantes. Construímos então uma combinação invariante de perturbações para uma perturbação de tensor de segunda ordem tal como $\delta G$ que o seja. Para tanto, utilizamos o tensor de Einstein não perturbado para uma secção espacial plana e com tempo conforme:

$$
{ }^{(0)} G_{0}^{0}=\frac{3 \mathcal{H}^{2}}{a^{2}}, \quad{ }^{(0)} G_{i}^{0}=0 \quad \text { e }{ }^{(0)} G_{j}^{i}=\frac{1}{a^{2}}\left(2 \mathcal{H}^{\prime}+\mathcal{H}^{2}\right) \delta_{i j}
$$

onde $\mathcal{H} \equiv \frac{a^{\prime}}{a}$, ' denotando a derivada com respeito ao tempo conforme. O seguinte objeto é então invariante:

$$
\begin{aligned}
& \delta \bar{G}_{0}^{0}=\delta G_{0}^{0}-\left({ }^{(0)} G_{0}^{0}\right)^{\prime}\left(B-E^{\prime}\right), \\
& \delta \bar{G}_{i}^{0}=\delta G_{i}^{0}-\left({ }^{(0)} G_{0}^{0}-^{(0)} G_{k}^{k} / 3\right)\left(B-E^{\prime}\right)_{, i}, \\
& \delta \bar{G}_{j}^{i}=\delta G_{0}^{0}-\left({ }^{(0)} G_{j}^{i}\right)^{\prime}\left(B-E^{\prime}\right),
\end{aligned}
$$

${ }^{(0)} G_{k}^{k}$ representando o traço das componentes espaciais.

Desse modo as equações invariantes para perturbações são:

$$
\bar{\delta}_{\beta}^{\alpha}=8 \pi G \delta \bar{T}_{\beta}^{\alpha}
$$


Extraímos então as equações para cada uma das componentes invariantes:

$$
\begin{gathered}
\Delta \Psi-3 \mathcal{H}\left(\Psi^{\prime}+\mathcal{H} \Phi\right)=4 \pi G a^{2} \delta \bar{T}_{0(S)}^{0}, \\
\left(\Psi^{\prime}+\mathcal{H} \Phi\right)_{, i}=4 \pi G a^{2} \delta \bar{T}_{i(S)}^{0}, \\
{\left[\Psi^{\prime \prime}+\mathcal{H}(2 \Psi+\Phi)^{\prime}+\left(2 \mathcal{H}^{\prime}+\mathcal{H}^{2}\right) \Phi+\frac{1}{2} \Delta(\Phi-\Psi)\right] \delta_{i j}-\frac{1}{2}(\Phi-\Psi)_{, i j}=-4 \pi G a^{2} \delta \bar{T}_{j(S)}^{i},}
\end{gathered}
$$

para perturbações escalares, onde o subíndice ${ }_{(S)}$ significa parte escalar;

$$
\begin{gathered}
\Delta \bar{V}_{i}=16 \pi G a^{2} \delta \bar{T}_{i(V)}^{0} \\
\left(\bar{V}_{i, j}+\bar{V}_{j, i}\right)^{\prime}+2 \mathcal{H}\left(\bar{V}_{i, j}+\bar{V}_{j, i}\right)=-16 \pi G a^{2} \delta \bar{T}_{j(V)}^{i},
\end{gathered}
$$

para perturbações vetoriais, sendo que o subíndice $(V)$ denota a parte vetorial da perturbação invariante;

$$
\left(h_{i j}^{\prime \prime}+2 \mathcal{H} h_{i j}^{\prime}-\Delta h_{i j}\right)=16 \pi G a^{2} \delta \bar{T}_{j(T)}^{i},
$$

para perturbações tensoriais, em que o subíndice ${ }_{(T)}$ denota a parte tensorial de $\delta T$.

\subsection{Geração de perturbações}

No capítulo anterior, tratamos a inflação dirigida por campo escalar em ordem zero de perturbação e concluímos que a estrutura de background do universo observado se torna ponto atrator da dinâmica. Reincorporando-se as perturbações do campo $\phi, \delta \phi(x, t)$, conclui-se que, a nível clássico, o paradigma inflacionário não leva a previsões adicionais além de uma universo perfeitamente homogêneo em escalas interiores ao horizonte. Essa situação, contudo, se inverte numa descrição semiclássica.

\subsubsection{A evolução clássica das perturbações durante a inflação}

Consideremos a perturbação do campo inflaton, $\delta \phi(x, t)$, no tensor energia-momento 3.47. Como argumentado, necessitamos de uma quantidade invariante de calibre caracterizando a perturbação em $\phi$. Considerando o lema de Stewart-Walker 4.30 e as regras de transformação 4.3 , conclui-se que

$$
\overline{\delta \phi}=\delta \phi-\phi_{0}^{\prime}\left(B-E^{\prime}\right)
$$


é invariante. Esta quantidade é simplesmente $\delta \phi$ no calibre longitudinal.

O importante a observar é que a perturbação do tensor energia-momento 3.47 contém apenas componentes escalares, como se poderia esperar já pelo fato de serem funções de um único componente escalar, $\delta \phi$. Desse modo, o problema resume-se na consideração das componentes $\Psi, \Phi$ e $\bar{\delta} \phi$. Necessitamos, por tanto, de três equações. Uma delas pode ser obtida perturbando a equação de Euler-Lagrange derivada da ação 3.46, a saber:

$$
\frac{1}{\sqrt{-g}} \frac{\partial}{\partial x^{\alpha}}\left(\sqrt{-g} g^{\alpha \beta} \frac{\partial \phi}{\partial x^{\beta}}\right)+\frac{\partial V}{\partial \phi}=0 .
$$

A equação resultante é:

$$
\delta \phi^{\prime \prime}+2 \mathcal{H} \delta \phi^{\prime}-\nabla \delta \phi+a^{2} V_{, \phi \phi} \delta \phi-\phi_{0}^{\prime}(3 \Psi+\Phi)^{\prime}+2 a^{2} V_{, \phi} \Phi=0
$$

A seguinte pode ser obtida de

$$
\overline{\delta T}_{i}^{0}=\frac{1}{a^{2}}\left(\phi_{0}^{\prime} \overline{\delta \phi}\right)_{, i}
$$

e 4.44, que nos leva a:

$$
\Psi^{\prime}+\mathcal{H} \Phi=4 \pi \phi_{0}^{\prime} \overline{\delta \phi}
$$

enquanto a última equação vem de $\delta \bar{T}_{j}^{i}=0$, que com 4.45 implica em

$$
\Phi=\Psi
$$

A equação 4.51 pode ser versada numa equação diferencial ordinária pela transformada de Fourier e resolvida em dois regimes: o de pequenos comprimentos de onda, $\lambda_{p h} \ll H^{-1}$, o que equivale a $k \gg H a$, e o de grandes comprimentos de onda, $\lambda_{p h} \gg H^{-1}$. Aqui, $\lambda_{p h}$ representa o comprimento de onda físico, isto é, da onda descrita em termos das coordenadas $\bar{x}^{i}=a(t) \cdot x^{i}$, ou seja, da onda descrita na base $e^{i \bar{k}_{j} \bar{x}^{j}}, x^{i}$ definido tal que a métrica se escreve $d s^{2}=d t^{2}-a^{2}(t)\left[\left(d x^{1}\right)^{2}+\left(d x^{2}\right)^{2}+\left(d x^{3}\right)^{2}\right]$, enquanto $k$ é vetor de onda comóvel, isto é, da onda descrita em termos das coordenadas $x^{i}$.

Em pequenas escalas, as perturbações do potencial gravitacional não devem afetar a evolução da perturbação $\delta \phi$. Considerando ainda a condição de slow-roll $V_{, \phi \phi} \ll V \sim H^{2}$, temos que 4.51 se reduz a

$$
\delta \phi_{k}^{\prime \prime}+2 \mathcal{H} \delta \phi_{k}^{\prime}+k^{2} \delta \phi_{k}=0
$$

que tem como solução

$$
\delta \phi_{k} \approx \frac{C_{k}}{a} \exp ( \pm i k \eta)
$$


Essa solução nos mostra que, em escalas que se originam no interior do horizonte de Hubble e cujo comprimento de onda é exponencialmente ampliado até cruzar o horizonte, existe uma supressão da amplitude clássica original que é tão maior quanto menor for esta em relação ao tamanho do horizonte. Como veremos adiante, o mesmo não ocorre com a perturbação $\Psi$, que apenas oscila em pequena escala. Essa situação, contudo, não altera o efeito de supressão inflacionária de inomogeneidades em pequena escala a nível clássico. De fato, isso decorre do lema de Riemann-Lebesgue Friedlander e Joshi (1998), aplicável a um campo pré-inflacionário inicial que é clássico, contínuo e de decrescimento suficientemente rápido, de acordo com o requerido pelas condições de contorno que asseguram a unicidade da decomposição STV:

$$
\lim _{|k| \rightarrow \infty} \int d^{3} x e^{i k x} \Psi(x, t)=0 .
$$

Em outras palavras, desde que a inflação ao menos preserve a amplitude da perturbação numa escala física pequena ao longo de sua evolução, o universo, em escalas comparáveis ao horizonte de Hubble, terminará dominado por componentes que no início da inflação tinham $k$ arbitrariamente grande, implicando em amplitudes arbitrariamente pequenas pelo lema acima.

Quanticamente, porém, o lema de Riemann-Lebesgue não se aplica. Contrariamente, as flutuações quânticas, presentes como correções de quaisquer trajetórias clássicas, têm amplitude tanto maior quanto menor a escala considerada. De fato, considerando, por exemplo, o campo escalar livre $\phi$ no espaço-tempo plano e lagrangiana:

$$
L=\frac{1}{2} \int d^{4} x\left(\partial^{\mu} \phi \partial_{\mu} \phi-m^{2} \phi^{2}\right)
$$

submetido às regras usuais de quantização, nos leva a uma função de correlação no estado de menor energia, o vácuo, da forma:

$$
\langle 0|\phi(t, x) \phi(t, y)| 0\rangle=\xi_{\phi}(|x-y|)=\frac{1}{4 \pi^{2}} \int \frac{k^{3}}{\omega_{k}} \frac{\sin (k|x-y|)}{k|x-y|} \frac{d k}{k},
$$

sendo $\omega_{k}=\sqrt{k^{2}+m^{2}}$, que, comparando-se a 4.15 , nos leva a uma variância adimensional da forma:

$$
\delta \phi_{L}^{2}=\frac{1}{4 \pi^{2}} \frac{k^{3}}{\omega_{k}}
$$

que, no limite de pequenos comprimentos de onda, implica $\delta \phi_{L}=\sqrt{\delta \phi_{L}^{2}}$, um caracterizador da amplitude de flutuação, inversamente proporcional à escala $L$. 
A consequência disso, numa descrição semiclássica de perturbações, é que as flutuações quânticas dominam a amplitude de perturbações no intervalo de escalas comparável ao tamanho do horizonte ao fim da inflação.

Para estudar o comportamento da amplitude de perturbação após cruzar o horizonte, consideremos a aproximação de slow-roll na evolução de background:

$$
\ddot{\phi_{0}}+3 H \dot{\phi}_{0}+V_{, \phi}=0
$$

que se simplifica em:

$$
3 H \dot{\phi}+V_{, \phi} \approx 0
$$

Escrevendo 4.51 e 4.53 em termos do tempo ordinário, ao invés do tempo conforme, resulta em:

$$
\begin{gathered}
\ddot{\delta \phi}+3 H \dot{\delta \phi}-\Delta \delta \phi+V_{, \phi \phi} \delta \phi-4 \dot{\phi}_{0} \dot{\Phi}+2 V_{, \phi} \Phi=0, \\
\dot{\Phi}+H \Phi=4 \pi \dot{\phi}_{0} \delta \phi
\end{gathered}
$$

O rolamento lento nos permite uma simplificação adicional nessas equações, ignorando os termos $\ddot{\delta} \phi$, uma suposição baseada na condição 3.56 , e $\dot{\Phi}$, que significa uma pequena mudança fracional em $\Phi_{k}$ num tempo de Hubble, uma condição que deve ser demonstrada autoconsistente em retrospectiva. Temos ainda que em grandes comprimentos de onda, o termo $\Delta \delta \phi$, ou melhor, sua contrapartida no espaço de Fourier, $-k^{2} \phi_{k}$, é desprezível. Adicionalmente, com a substituição: $y=\frac{\delta \phi}{V, \phi}$, temos:

$$
3 H \dot{y}+2 \Phi=0, \quad H \Phi=4 \pi \dot{V} y
$$

que nos leva a

$$
\delta \phi_{k}=A_{k} \frac{V_{, \phi}}{V}, \quad \Phi_{k}=-\frac{1}{2} A_{k}\left(\frac{V_{, \phi}}{V}\right)^{2}
$$

A solução acima nos mostra que, no limite de grandes comprimentos de onda em relação ao tamanho do horizonte, a amplitude das perturbações aumenta, ao invés de diminuir, na medida em que a inflação prossegue, pois o parâmetro de slow-roll, $\frac{V, \phi}{V}$, aumenta rumo à unidade, o que caracteriza o fim do rolamento lento e, por conseguinte, da inflação. Mais ainda, a amplitude muda por um fator multiplicativo independente de $k$. Ou seja, o padrão de flutuações, caracterizado pela amplitude relativa típica, isto é, a razão de 
amplitudes típicas entre uma dada escala e uma escala arbitrária de referência $\rrbracket^{8}$ em função do comprimento de onda, não mais se altera ao longo da evolução cósmica para um intervalo de escalas já maior que o horizonte. Isso caracteriza o fato da física causal ser inoperante em escalas muito maiores que o horizonte e de que um mecanismo de geração de perturbações deve afetar a amplitude numa dada escala apenas até a sua saída.

\subsubsection{A teoria quântica de perturbações cosmológicas}

De acordo com o exposto, ao fim da inflação, em escalas comparáveis ao horizonte e além, a teoria clássica de perturbações não prevê nada além de um universo perfeitamente homogêneo. Para ganhar em primeira ordem de perturbação a mesma previsibilidade que a inflação tem em ordem zero, devemos partir para uma descrição semiclássica. Que esse nível de descrição nos levaria a previsões adicionais, poderíamos supor já do fato de que flutuações quânticas serem maiores em menores escalas, escalas essas que se tornam as mais relevantes ao fim da inflação, na medida que horizonte comóvel, a escala de referência em modelos FRW, diminui exponencialmente. Para tanto, necessitamos quantizar $\Psi(x, t)$ e $\bar{\delta} \phi(x, t)$. Quantizar uma teoria clássica significa mapear observáveis clássicas, denotadas $\mathcal{O}$, em observáveis quânticas, denotadas $\hat{\mathcal{O}}$, de tal modo que o valor esperado de $\hat{\mathcal{O}}(t)$ em certos estados $|\psi\rangle$, chamados de semiclássicos, tenha como limite $\mathcal{O}(t)$ quando $\hbar \rightarrow 0$. Esta prescrição, embora não determine unicamente a regra de quantização, ressalta o papel da dinâmica na elaboração do método. Os esquemas de quantização disponíveis não são únicos e, além disso, ainda necessitam de uma demonstração de equivalência. O mais bem entendido destes métodos, o de quantização canônica, não é diretamente aplicável, dado o conjunto de equações dinâmicas de que dispomos.

Desejamos então mapear $\Psi(x, t)$ e $\bar{\delta} \phi(x, t)$ nas suas contrapartidas no espaço de Hilbert: $\hat{\Psi}(x, t)$ e $\hat{\bar{\phi}}(x, t)$. Poderíamos pensar em $\hat{\Psi}(x, t)$ ou $\hat{\bar{\phi}}(x, t)$ como regras que associam a cada conjunto de coordenadas, $(x, t)$, um operador autoadjunto no espaço de Hilbert. Em retrospectiva, contudo, essa expectativa é demasiado simplista. O que os métodos de quantização nos devolvem é uma regra que associa a cada função $f(x, t)$ infinitamente diferenciável e de rápido decrescimento 9 , um operador autoadjunto (quando os campos

\footnotetext{
${ }^{8}$ A teoria inflacionária não prevê a amplitude típica de perturbações, apenas a amplitude relativa. A amplitude observada, por exemplo, na CMB, é então um vínculo para os parâmetros livres do modelo

${ }^{9} \mathrm{O}$ que siginifica $\lim _{\|(x, t)\| \rightarrow \infty}\|(x, t)\|^{n} f=0$, para todo $n,\|\cdot\|$ denotando a norma euclidiana
} 
são de valor real) que seria o análogo quântico da expressão $\int d^{4} x f(x, t) \Psi(x, t)$, onde cada expressão desse tipo está associada a um operador definido no mesmo domínio $\mathcal{D}$ no espaço de Hilbert $\mathcal{H}$, que pode ser demonstrado sempre menor que $\mathcal{H}[10$ mas que pode ser escolhido denso, isto é, qualquer elemento de $\mathcal{H}$ pode ser obtido como limite de uma sequência construída por elementos de $\mathcal{D}$. Essa noção generaliza a função de valor operador, um operador para cada coordenada $(x, t)$, para a distribuição de valor operador. Esta é análoga a generalização que as distribuições, como a delta de Dirac, representam para as funções ordinárias, associando um valor real, não a cada ponto do domínio $\mathbb{R}^{n}$, mas sim a cada expressão do tipo $\int d^{4} x f(x, t) \Psi(x, t)$ 11 , para todo $f$ adequando, ainda que não exista uma função $\Psi(x, t)$ que satisfaça tais requisitos. Embora essa possa parecer uma mera tecnicalidade sem relevância física, é um dos principais problemas da teoria quântica de campos ao se tentar construir operadores derivados do campo quântico, os quais são as observáveis associadas ao campo: o produto de operadores no mesmo ponto não está a priori definido. Propriedade esta herdada de um teorema para distribuições, também devido a Swartz, o qual diz que é impossível definir um produto associativo (ou seja, que satisfaça $a(b c)=(a b) c$ ) e distributivo (que satisfaça $a(b+c)=a b+a c$ ) no espaço das distribuições e está ligado até ao problema da constante cosmológica, visto que a regra de quantização para os campos não determina unicamente as observáveis construídas a partir do campo tais como a energia ou densidade de energia.

Para definir a quantização canônica, primeiramente descrevemos a prescrição de Dirac para um número finito de graus de liberdade, a qual denotaremos por $\mathcal{Q}$. Esta é baseada na existência do limite semiclássico e, para tanto, pressupõe o formalismo Hamiltoniano como plano de fundo (veja a formulação de álgebra $C^{*}$ para teoria quântica no capítulo 6 para

\footnotetext{
${ }^{10} \mathrm{O}$ motivo para isso é que os operadores são autoadjuntos e ilimitados, onde ilimitado significa que não existe uma constante $C$ tal que $\|A x\| \leq C\|x\|$, propriedade que decorre das relações de comutação satisfeitas. Operadores autoadjuntos têm o gráfico fechado. O gráfico de um operador $A$ é o conjunto dos pares $(x, A x)$. Dizer que é fechado, quer dizer que todas as sequências convergentes construídas com elementos do gráfico convergem para elementos do gráfico. A sequência convergente é definida pela norma produto, $\|(x, A, x)\|=\sqrt{\|x\|^{2}+\|A x\|^{2}}$, ou seja, uma sequência $a_{n}=\left(x_{n}, A x_{n}\right)$ converge se e somente se $\left\|a_{n+m}-a_{n}\right\| \rightarrow 0$ quando $n \rightarrow \infty$ para qualquer $m \geq 1$ ). Operadores de gráfico fechado definidos num domínio fechado, como o espaço de Hilbert, são limitados, pelo teorema do Gráfico fechado.

${ }^{11}$ Acrescido de uma noção de continuidade que é definida a partir da regra que determina quando a sequência de funções teste $\Psi(x, t)$ converge para zero, isto é, a topologia do espaço de funções teste
} 
uma justificativa deste formalismo): O domínio de $\mathcal{Q}$ é o conjunto de todas as observáveis clássicas a princípio, representado por cada função $\mathcal{O}\left(q_{n}, p_{n}\right)$ das variáveis canônicas $q_{n}$ e $p_{n}$, coletivamente denotadas por $\mathcal{P}$. A operação relevante para a dinâmica é o parênteses de Poisson, o qual entra nas equações de Hamilton para a dinâmica: dado $f$ e $g \in \mathcal{P}$,

$$
\{f, g\}=\sum_{n} \frac{\partial f}{\partial q_{n}} \frac{\partial g}{\partial p_{n}}-\frac{\partial f}{\partial p_{n}} \frac{\partial g}{\partial q_{n}} .
$$

Uma vez que combinações lineares podem ser construídas entre elementos de $\mathcal{P}$, permanecendo ainda em $\mathcal{P}$ e a operação acima pode ser entendida como um produto, com resultado em $\mathcal{P}$, podemos definir $\mathcal{P}$ como uma álgebra, uma álgebra de Lie chamada de álgebra de Poisson, da qual $h(2 n)$ é uma subálgebra chamada de álgebra de Heisenberg ${ }^{12}$,

$$
h(2 n)=\operatorname{span}\left\{1, q_{n}, p_{n} \mid n=1, \ldots, n\right\}
$$

A vantagem conceitual de se falar em álgebra é que o problema fundamental da teoria quântica, quantizar, é então um problema para álgebra: Construir a representação de uma álgebra, isto é, conhecidas as operações entre os elementos, determinar um conjunto de operadores que as satisfaçam e avaliar a unicidade da teoria quântica correspondente.

Enunciemos então a prescrição de Dirac, para quantização de sistemas Hamiltonianos, como um problema algébrico: $\mathcal{Q}$, a operação que mapeia o elemento abstrato na sua representação concreta, é um operador linear do espaço $\mathcal{P}$ no espaço $O p(\mathcal{D})$ de operadores simétricos num domínio $D$ denso num espaço de Hilbert separável que satisfaz:

$\mathcal{Q}$ 1. $\mathcal{Q}(\{f, g\})=\frac{1}{i \hbar}[\mathcal{Q}(f), \mathcal{Q}(g)]$

Q 2. $\mathcal{Q}(1)=I$;

$\mathcal{Q}$ 3. Se e campo vetorial Hamiltoniano $X_{f}=\left(\frac{\partial f}{\partial p},-\frac{\partial f}{\partial q}\right)$ é completo, isto é, a solução das equações de movimento com Hamiltoniano $f$ se estende para todo t, $\mathcal{Q}$ é essencialmente autoadjunto em $\mathcal{D}$;

$\mathcal{Q}$ 4. $\mathcal{Q}$ representa $h(2 n)$ irredutivelmente;

$\mathcal{Q}$ 5. $\mathcal{D}$ contem um conjunto denso de vetores analíticos da base de $\mathcal{Q}(h(2 n))$

Observamos aqui três conceitos distintos que no espaço de Hilbert de dimensão infinita se resumem ao mesmo conceito, mas que devido a impossibilidade, em geral, de se

\footnotetext{
12 spam denota que um elemento da álgebra é uma combinação linear dos elementos listados
} 
estender livremente o domínio de um operador autoadjunto (segundo o footnote 10), são conceitos distintos em dimensão infinita: operador simétrico, essencialmente autoadjunto e autoadjunte ${ }^{13}$. Um espaço de Hilbert separável significa enumerabilidade da base, ou seja, podemos colocar os elementos de uma base de estados normalizáveis numa sequência. Fisicamente, isso significa que partindo de um autoestado normalizado qualquer rotulado por um conjunto de números quânticos, o sistema físico pode realizar transições para todos os demais estados. $\mathcal{Q} 1$. e $\mathcal{Q} 2$. estão associados à existência do limite semiclássico (veja discussão no capítulo 6). Q23. significa que uma função $f\left(q_{n}, p_{n}\right)$ pode ser estendida a uma observável quântica se for, a princípio, uma boa Hamiltoniana levando a comportamentos não singulares ${ }^{14}$, $\mathcal{Q} 4$. está associado à unicidade do problema de representação segundo o teorema de Von Neumann, que estabelece a equivalência das representações irredutíveis da álgebra de Heisenberg (veja capítulo 6), o conceito de irredutibilidade significa que a representação não pode ser tornada mais simples, que não existe nenhum subespaço de $\mathcal{H}$ que é deixado invariante pela ação de $e^{i k_{i} p_{i}}, e^{l_{j} q_{j}}, k_{i}, l_{j} \in \mathbb{R}$ (veja 5.2 .3 do capítulo 5 para os conceitos de irredutibilidade e equivalência de representações). $\mathcal{Q} 5$. diz que existe uma base na qual podemos aplicar livremente $\mathcal{Q}(h(2 n))$ e suas exponenciais, o que está ligado à possibilidade de construir observáveis físicas a partir da posição e momento.

A generalização de 4.67 para campos se escreve mapeando $q_{n}(t)$ no campo $\phi_{n}(t, x)$ e $p_{n}(t)$ no campo $\pi_{n}(t, x)$, as funções $f\left(q_{n}, p_{n}\right)$ nos funcionais $\mathcal{F}[\phi, \pi]$ e definido:

$$
\left\{\mathcal{F}\left[\phi_{n}, \pi_{n}\right], \mathcal{G}\left[\phi_{n}, \pi_{n}\right]\right\}=\sum_{n}\left\{\frac{\delta \mathcal{F}}{\delta \phi_{n}(x, t)} \frac{\delta \mathcal{G}}{\delta \pi_{n}(x, t)}-\frac{\delta \mathcal{F}}{\delta \pi_{n}(x, t)} \frac{\delta \mathcal{G}}{\delta \phi_{n}(x, t)}\right\}
$$

\footnotetext{
${ }^{13}$ Dado um operador linear $A$ com domínio no subespaço $\mathcal{D}$ no espaço de Hilbert $\mathcal{H}$, a adjunta $A^{*}$ é o operador definido no subespaço $\mathcal{D}^{\prime}$ dos elementos $\xi \in \mathcal{H}$ tais que $(\xi, A \psi) \leq C\|\psi\|$. Isto implica, pelo teorema de Riesz, que existe um único $A^{*} \xi$ tal que $\left(A^{*} \xi, \psi\right)=(\xi, A \psi)$. Quando $A=A^{*}$, o que também necessita $\mathcal{D}=\mathcal{D}^{\prime}, A$ é autoadjunto. Se $\mathcal{D}^{\prime} \supseteq \mathcal{D}$ e $A=A^{*}$ em $\mathcal{D}, A$ é dito simétrico. Por fim, suponha $A$ fechável, isto é, se o fechamento do gráfico de $A$ (incorporação, no gráfico, de todos os elementos que podem ser obtidos por limites de sequências no gráfico), segundo a definição dada no footnote 10 , definir o gráfico de um operador $\bar{A}$ tal que $\bar{A}=A^{*}, A$ é essencialmente autoadjunto. A é fechável se e somente se para toda sequência $x_{n} \rightarrow 0, x_{n} \in \mathcal{D}$, então $A x_{n} \rightarrow 0$

${ }^{14}$ Uma solução das equações de Hamilton que não esteja definida para todo $t$ pode a princípio divergir para um $t$ finito. Esse tipo de comportamento contradiz a existência de soluções quânticas definidas para todo $t$ : a evolução temporal dos autoestados de $H$, estados estes que formam uma base para a definição de todas as soluções.
} 
Q1. é então aplicável se as equações de campo forem da forma Hamiltoniana.

$$
\begin{aligned}
& \dot{\phi}=\{\phi, H\} \\
& \dot{\pi}=\{\pi, H\}
\end{aligned}
$$

Na verdade, a propriedade $\mathcal{Q} 1$ acima, conforme a ideia original de Dirac, não pode ser satisfeita para polinômios em $q_{n}$ e $p_{n}$ de ordem mais elevada que dois. Esse é o conteúdo do teorema de Groenewold-Van Hove $€^{15}$, devendo ser substituída por:

$\mathcal{Q}$ 1. $\mathcal{Q}(\{f, g\})=\frac{1}{i \hbar}[\mathcal{Q}(f), \mathcal{Q}(g)]+\mathcal{O}(\hbar)$

onde $\mathcal{O}(\hbar)$ refere-se à constante $C$ na definição de operador limitado no footnote 10 .

Necessitamos então de uma combinação linear de $\Psi$ e $\overline{\delta \phi}$, de modo a manter a linearidade das equações, que obedeça as equações de Hamilton. Estas são:

$$
u \equiv \frac{\Psi}{4 \pi(\epsilon+p)^{\frac{1}{2}}} \quad \text { e } \quad v \equiv \sqrt{\epsilon_{, X}}\left(\overline{\delta \phi}+\frac{\phi_{0}^{\prime}}{\mathcal{H}} \Psi\right)
$$

onde assumimos que a perturbação $\overline{\delta \phi}$ é a perturbação de um campo de lagrangiana

$$
\int p(X, \phi) \sqrt{-g} d^{4} x
$$

sendo $X=\frac{1}{2} g^{\alpha \beta} \phi_{, \alpha} \phi_{, \beta}$, o que nos leva a um tensor energia-momento da forma de um fluido perfeito:

$$
T_{\beta}^{\alpha}=(\epsilon+p) u^{\alpha} u_{\beta}-p \delta_{\beta}^{\alpha}
$$

$\operatorname{com} u_{\alpha} \equiv 2 X p_{, X}-p$.

4.72 satisfazem as equações:

$$
c_{s} \Delta u=z\left(\frac{v}{z}\right)^{\prime} \quad \text { e } \quad c_{s} v=\theta\left(\frac{u}{\theta}\right)^{\prime}
$$

onde

$$
z \equiv \frac{a^{2}(\epsilon+p)^{\frac{1}{2}}}{c_{s} \mathcal{H}}, \quad \theta \equiv \frac{1}{c_{s} z}
$$

15 De fato, um exemplo, não uma demonstração, pode ser obtido assumindo $\mathcal{Q} 1$. válido para variáveis canônicas e estendendo para polinômios a partir da simetrização. Temos que $\frac{1}{9}\left\{q^{3}, p^{3}\right\}=q^{2} p^{2}=$ $\frac{1}{3}\left\{q^{2} p, p^{2} q\right\}$, enquanto $\frac{1}{9}\left[\mathcal{Q}\left(q^{3}\right), \mathcal{Q}\left(p^{3}\right)\right]=\mathcal{Q}\left(q^{2}\right) \mathcal{Q}\left(p^{2}\right)-2 i \hbar \mathcal{Q}(q) \mathcal{Q}(p)-\frac{2}{3} \hbar^{2} I$ e $\frac{1}{3}\left[\mathcal{Q}\left(q^{2} p\right), \mathcal{Q}\left(p^{2} q\right)\right]=$ $\mathcal{Q}\left(q^{2}\right) \mathcal{Q}\left(p^{2}\right)-2 i \hbar \mathcal{Q}(q) \mathcal{Q}(p)-\frac{1}{3} \hbar^{2} I$. 
em que $c_{s}$ é a velocidade do som no fluido:

$$
c_{s}^{2}=\frac{p_{, X}}{\epsilon_{, X}}
$$

4.75 implicam em:

$$
\begin{aligned}
& u^{\prime \prime}-c_{s}^{2} \Delta u-\frac{\theta^{\prime \prime}}{\theta} u=0 \\
& v^{\prime \prime}-c_{s}^{2} \Delta v-\frac{z^{\prime \prime}}{z} v=0
\end{aligned}
$$

que podem então ser deduzidos das lagrangianas:

$$
\begin{aligned}
S_{u} & \equiv \int \mathcal{L}_{u} d \eta d^{3} x=\frac{1}{2} \int\left(u^{\prime 2}+c_{s}^{2} u \Delta u+\frac{\theta^{\prime \prime}}{\theta} u^{2}\right) d \eta d^{3} x \\
S_{v} & \equiv \int \mathcal{L}_{v} d \eta d^{3} x=\frac{1}{2} \int\left(v^{\prime 2}+c_{s}^{2} v \Delta v+\frac{z^{\prime \prime}}{z} v^{2}\right) d \eta d^{3} x
\end{aligned}
$$

levando a momentos canonicamente conjugados:

$$
\pi_{u}=\frac{\partial \mathcal{L}}{\partial u^{\prime}}, \quad \pi_{v}=\frac{\partial \mathcal{L}}{\partial v^{\prime}}
$$

em que a Hamiltoniana é dada pela transformada de Legendre. A quantização é dada por $\mathcal{Q} 1$ e 4.69 . Outro ponto em que o caso de graus de liberdade infinitos se distingue do caso de graus de liberdade finito é que o requerimento $\mathcal{Q} 4$ não mais é suficiente para garantir a unicidade (a menos de uma transformação unitária) da representação. Na verdade, as inequivalentes representações de

$$
\begin{gathered}
{[\hat{v}(\eta, x), \hat{v}(\eta, y)]=\left[\hat{\pi}_{v}(\eta, x), \pi_{v}(\eta, y)\right]=0} \\
{\left[\hat{v}(\eta, x), \pi_{v}(\eta, y)\right]=i \delta(x-y)}
\end{gathered}
$$

desafiam qualquer tentativa de classificação. Isto se manifesta no objeto que desejamos calcular, a função de correlação de dois pontos:

$$
\langle 0|\Psi(\eta, x) \Psi(\eta, y)| 0\rangle
$$

Mais geralmente, a inequivalência das representações se manifesta na inequivalência das funções de $N$-pontos. De fato, discutiremos mais à frente o teorema de Wigthman, o qual diz que o conhecimento de todas as funções de $N$-pontos no vácuo tem informação suficiente para construir a representação dos campos para além de qualquer problema de 
unicidade, se assumirmos a unicidade e ciclicidade ${ }^{16}$ do vácuo. A premissa fundamental da teoria quântica de perturbações cosmológicas é substituir a função de correlação de $N$-pontos estatística pela função de $N$-pontos em algum estado adequado, que é escolhido como um dos possíveis estados de vácuo, primeiramente por estarmos interessados no menor nível possível de flutuações, segundo, por refletir o estado clássico de ausência total de perturbações, ou seja, valor esperado das perturbações é zero no momento em que as perturbações se tornam clássicas. A escolha de funções de $N$-pontos diferentes significa a escolha de representações inequivalentes, mas usualmente é referido como a escolha de um estado de vácuo diferente. O teorema de Wick, que vale para campos livres, de equações lineares, nos diz que a função de $N$-pontos se fatora em termos da função de dois pontos, de modo que esta é a única com que precisamos nos preocupar.

$$
\hat{v}(\eta, x)=\frac{1}{\sqrt{2}} \int \frac{d^{3} k}{(2 \pi)^{\frac{3}{2}}}\left[v_{k}^{*}(\eta) e^{i k x} \hat{a}_{k}^{-}+v_{k}(\eta) e^{-i k x} \hat{a}_{k}^{+}\right]
$$

onde

$$
v_{k}^{\prime \prime}+\omega_{k}^{2}(\eta) v_{k}=0 \quad \text { e } \quad \omega_{k}^{2}(\eta)=c_{s}^{2} k^{2}-\frac{z^{\prime \prime}}{z}
$$

Os operadores $\hat{a}_{k}^{-}$e $\hat{a}_{k}^{+}$podem ser escolhidos satisfazendo as usuais relações de comutação bosônicas,

$$
\left[\hat{a}_{k}^{-}, \hat{a}_{k^{\prime}}^{-}\right]=\left[\hat{a}_{k}^{+}, \hat{a}_{k^{\prime}}^{+}\right]=0 \quad \text { e }\left[\hat{a}_{k}^{-}, \hat{a}_{k^{\prime}}^{+}\right]=\delta\left(k-k^{\prime}\right)
$$

se impusermos a condição

$$
v_{k}^{\prime} v_{k}^{*}-v_{k} v_{k}^{*^{\prime}}=2 i
$$

Uma vez que 4.89 é o Wronskiano de equação 4.87, $v_{k}$ e $v_{k}^{*}$ são soluções independentes de 4.87. Fazendo $v_{k}=r_{k} \exp \left(i \alpha_{k}\right)$, 4.89 se reduz a:

$$
r_{k}^{2} \alpha_{k}^{\prime}=1
$$

É nesse momento que necessitamos de um critério adicional para a escolha do vácuo. Para tanto, escrevamos o Hamiltoniano associado a $v$ :

$$
H=\int d^{3} x\left[\dot{v} \frac{\partial \mathcal{L}}{\partial \dot{v}}-\mathcal{L}\right]
$$

\footnotetext{
${ }^{16}$ A aplicação sucessiva de operadores de campo da forma $\int d \eta d^{3} x f(\eta, x) \Psi(\eta, x)$ no vácuo gera um subconjunto denso no espaço de Hilbert dos campos.
} 


$$
\begin{gathered}
\hat{H}(\eta)=\frac{1}{2} \int d^{3} x\left[\hat{\pi}^{2}+c_{s}^{2}(\nabla v)^{2}+m_{e f f}^{2}(\eta) v^{2}\right], \quad m_{e f f}^{2}=-\frac{z^{\prime \prime}}{z} \\
\hat{H}(\eta)=\frac{1}{2} \int d^{3} k\left[\hat{a}_{k}^{-} \hat{a}_{k}^{-} F_{k}^{*}+\hat{a}_{k}^{+} \hat{a}_{k}^{+} F_{k}+\left(2 \hat{a}_{k}^{+} \hat{a}_{k}^{-}+\delta^{3}(0)\right) E_{k}\right] \\
E_{k}=\frac{1}{2}\left(\left|v_{k}^{\prime}\right|^{2}+\omega_{k}^{2}(\eta)\left|v_{k}\right|^{2}\right)=\frac{1}{2}\left(r_{k}^{\prime 2}+\frac{1}{r_{k}^{2}}+\omega_{k}^{2} r_{k}^{2}\right), \\
F_{k}(\eta)=\frac{1}{2}\left(v_{k}^{\prime 2}+\omega_{k}^{2}(\eta) v_{k}^{2}\right),
\end{gathered}
$$

sendo $\delta^{3}(0)$ uma divergência associada ao já comentado problema do produto de campos no mesmo ponto não definido a priori. Este termo é associado a uma integral no volume infinito. Escolhemos o estado de vácuo de menor densidade energia no instante $\eta_{0},\left|0_{(v)}\right\rangle$ :

$$
\left\langle 0_{(v)}\left|\hat{H}\left(\eta_{0}\right)\right| 0_{(v)}\right\rangle=\frac{1}{2} \delta^{3}(0) \int d^{3} k E_{k}(\eta)
$$

que é também o estado de aniquilado pelos operadores de destruição $\hat{a}_{k}^{-}\left(\eta_{i}\right)$.

A minimização de $E_{k}$ em $\eta=\eta_{0}$, para todo $k$, exige $r_{k}^{\prime}\left(\eta_{i}\right)=0$ e $r_{k}\left(\eta_{i}\right)=\omega_{k}^{-\frac{1}{2}}$, que são então condições iniciais para as equações 4.87 que fixam a representação das relações de comutação 4.83 e 4.84 . Em termos de $v$, essas condições se escrevem como:

$$
v_{k}\left(\eta_{i}\right)=\frac{1}{\sqrt{\omega_{k}}} e^{i \alpha_{k}\left(\eta_{i}\right)}, \quad v_{k}^{\prime}\left(\eta_{i}\right)=i \sqrt{\omega_{k}} e^{i \alpha_{k}\left(\eta_{i}\right)} .
$$

Observe que apenas quando $\omega_{k}^{2}(\eta)=c_{s}^{2} k^{2}-\frac{z^{\prime \prime}}{z}>0$ a noção de estado instantâneo de mínima energia existe. As escalas mais relevantes ao fim da inflação são associadas aos maiores valores $k$, da ordem de grandeza do inverso da escala de Hubble comóvel, a qual diminui exponencialmente, validando a análise.

As condições 4.97 se traduzem em condições iniciais para $u$ via 4.75 , que no limite de pequenas escalas, $c_{s}^{2} k^{2} \gg\left(\frac{z^{\prime \prime}}{z}\right)_{i}$ :

$$
u_{k}\left(\eta_{i}\right) \approx-\frac{i}{\sqrt{c_{s}} k^{\frac{3}{2}}}, \quad u_{k}^{\prime}\left(\eta_{i}\right) \approx \frac{\sqrt{c_{s}}}{k^{\frac{1}{2}}}
$$

Por fim, via variável $u$ :

$$
\langle 0|\hat{\Phi}(\eta, x) \hat{\Phi}(\eta, y)| 0\rangle=\int 4(\epsilon+p)\left|u_{k}\right|^{2} k^{3} \frac{\sin k r}{k r} \frac{d k}{k}
$$

que nos leva, via 4.15, a:

$$
\delta_{\Phi}^{2}(k, \eta)=4(\epsilon+p)\left|u_{k}(\eta)\right|^{2} k^{3}
$$


O objetivo é calcular $\left|u_{k}(\eta)\right|$ para um $\eta$ suficientemente grande, numa época pós inflacionária, fixando as condições de contorno 4.98 no momento de cruzamento do horizonte $c_{s} k \approx H a$. Ou seja, estamos interessados numa solução de 4.78 no limite de grandes comprimentos de onda, $c_{s}^{2} k^{2} \ll \frac{\theta^{\prime \prime}}{\theta}$, que possui duas soluções linearmente independentes, sendo uma delas $\theta$ e a segunda obtida pelo Wronskiano:

$$
u=C_{1} \theta+C_{2} \theta \int_{\eta_{0}} \frac{d \eta}{\theta^{2}}
$$

Da definição de $\theta$, vemos que a primeira solução é exponencialmente suprimida. Aplicandose a definição de $\theta$ :

$$
u_{k}(\eta) \equiv \frac{\Phi}{\pi(\epsilon+p)^{\frac{1}{2}}}=\frac{A_{k}}{4 \pi(\epsilon+p)^{\frac{1}{2}}}\left(1-\frac{H}{a} \int a d t\right) .
$$

Uma vez que $H$ varia muito lentamente durante a inflação e:

$$
\frac{1}{a} \int a d t=\frac{1}{a} \int \frac{d a}{H}=H^{-1}-\frac{1}{a} \int \frac{d a}{H}\left(H^{-1}\right)^{\cdot}=H^{-1}\left[1-\left(H^{-1}\right)^{\cdot}+\left(H^{-1}\left(H^{-1}\right)^{\cdot}\right)^{\cdot}\right],
$$

onde $\left(\quad\right.$ ) representa a derivada temporal, a expressão para $u_{k}$ então se simplifica como:

$$
u_{k}(\eta) \approx-\frac{A_{k}}{4 \pi(\epsilon+p)^{\frac{1}{2}}}\left(\frac{\dot{H}}{H^{2}}\right)=A_{k} \frac{(\epsilon+p)^{\frac{1}{2}}}{H^{2}},
$$

que com as condições de contorno que especificam $A_{k}$, resulta em:

$$
\delta_{\Phi}^{2}(k, t) \approx \frac{16}{9}\left(\frac{\epsilon}{c_{s}\left(1+\frac{p}{\epsilon}\right)}\right)_{c_{s} k \approx H a}\left(1-\frac{H}{a} \int a d t\right)^{2}
$$

que, assumindo-se uma fase dominada por radiação posterior ao fim da inflação, $a \propto t^{\frac{1}{2}}$, resulta em:

$$
\delta_{\Phi}^{2} \approx \frac{64}{81}\left(\frac{\epsilon}{c_{s}\left(1+\frac{p}{\epsilon}\right)}\right)_{c_{s} k \approx H a}
$$


Capítulo 5

\section{O significado conceitual da relação de dispersão}

\subsection{Introdução}

A noção de que deve haver uma correspondência chamada quantização entre uma teoria clássica e uma teoria quântica, embora não completamente estabelecida para além de qualquer problema conceitual, dentre estas a questão da unicidade, ainda que de grande valor prático, nos leva a pensar que o ponto de partida da teoria quântica é necessariamente uma teoria clássica. Não existe, contudo, uma razão fundamental pela qual uma teoria quântica não pudesse a princípio ser formulada sem nenhuma referência a um cenário determinístico clássico. Esta ideia guiou Wigner na mudança de paradigma envolvida na construção da teoria de campos: a ideia de que o problema fundamental da teoria quântica relativística seria, não a quantização de uma teoria clássica, mas sim a construção de uma representação do grupo de Poincaré no espaço de Hilbert segundo o critério que define uma simetria quântica. Em outras palavras, dado o espaço de estados $\mathcal{H}$, ao invés de determinar a correspondência $\mathcal{Q}$, que define a quantização segundo discutido na seção anterior, determinar a correspondência $\pi$ entre a transformação de Lorentz $x \rightarrow \Lambda x+a^{\mu}$, denotada $\left(\Lambda, a^{\mu}\right)$, e a transformação $\pi\left[\left(\Lambda, a^{\mu}\right)\right]: \mathcal{H} \rightarrow \mathcal{H}$ que satisfaça:

$$
\begin{gathered}
\pi\left[\left(\Lambda_{1}, a_{1}^{\mu}\right)\right] \cdot \pi\left[\left(\Lambda_{2}, a_{2}^{\mu}\right)\right]=\pi\left[\left(\Lambda_{1}, a_{1}^{\mu}\right) \cdot\left(\Lambda_{2}, a_{2}^{\mu}\right)\right], \\
\pi\left[\left(\Lambda_{1}, a_{1}^{\mu}\right)\right]^{-1}=\pi\left[\left(\Lambda_{1}, a_{1}^{\mu}\right)^{-1}\right], \\
\pi\left[\left(\Lambda, a^{\mu}\right)\right] \text { é uma simetria quântica, }
\end{gathered}
$$

onde · denota a composição, transformação resultante da aplicação sucessiva de duas operações e ()$^{-1}$ a inversa. A caracterização de uma simetria é dada por um resultado devido a Wigner. A relação de dispersão, neste contexto, é uma peça fundamental da 
resolução deste problema.

Para confirmar o caráter fundamental das simetrias, observamos que a simples modificação desse grupo, de Galileu para o de Lorentz, cria não apenas uma fenomenologia completamente nova, associada, por exemplo, à criação e destruição de partículas, ligada à necessidade de se empregar representações altamente redutíveis do grupo de Poincaré para descrever interações, mas a sérias dificuldades técnicas como as discutidas na seção anterior, associadas à introdução do conceito de campo quântico, ligada a requerimentos de causalidade/localidade. Soma-se a isso a impossibilidade de definir um conceito relativístico de localização de partícula e, o que se demonstra mais grave, no problema de quantização, já antecipado por resultados fundamentais como o teorema de Haag, o qual diz que o formalismo de interação, ponto de partida da teoria de perturbações usual, representada pela fórmula de Gell-Mann Low, associada aos famosos diagramas de Fenyman, embora funcione sob ação do grupo Euclidiano de rotações e translações, não pode funcionar sob a hipótese relativística. Isso devido essencialmente ao fato de que as interações empregam realizações do grupo de Poincaré necessariamente inequivalentes àquelas em que o conceito de um conjunto de partículas não interagentes em algum instante está definido (estados assintóticos em que as partículas são consideradas livres antes de um evento de espalhamento). Na verdade, o teorema captura a noção intuitiva de que não existem partículas livres em teorias interagentes. A noção clássica de que podemos afastar duas partículas de modo que elas se tornem efetivamente livres é inválida, devido ao fato de que elétrons, por exemplo, estão sempre circundados por nuvens de fótons virtuais. Segundo o teorema, se o conceito de partículas livres está bem definido em algum instante qualquer do tempo e a teoria é relativística, ela é necessariamente livre. Os problemas relacionados ao teorema de Haag foram apenas em parte resolvidos pela renormalização.

As principais referências para esse capítulo são: Von Neumann (1996), de Faria e de Melo (2010), Weinberg (2005), Loewner (2008), Bogolubov et al. (1975), Debnath e Mikusinski (2005), Haag (1996), Balachandran e Trahern (1986) e Sakurai (1967). 


\subsection{O problema da realização de simetrias no contexto quântico}

\subsubsection{O teorema de Wigner}

Quando falamos sobre quantização de campos, definimos quantizar como associar uma teoria quântica a uma teoria clássica de tal modo que em determinado limite, o semiclássico, a teoria quântica obtêm a teoria clássica correspondente, ou seja, prevê o comportamento clássico. Mas não nos delongamos sobre uma definição apropriada para teoria quântica. Essa definição é dada em termos da estrutura matemática das observáveis numa teoria quântica, isto é, pelo que entendemos por estados e observáveis, através axiomas de Von Neumann (veja no capítulo 6 que a descrição de observáveis físicas por operadores autoadjuntos no espaço de Hilbert é na verdade uma liberdade de formulação semelhante àquela da formulação tensorial):

1. Estados puros são raios (vetores normalizados, a menos de uma fase) no espaço de Hilbert $\mathcal{H}$ complexo e separável;

2. Observáveis são operadores autoadjuntos (densamente) definidos no espaço de Hilbert $\mathcal{H}$;

3. Dado um conjunto de Borel $E$ [1 a probabilidade de uma observável $A$ ter um valor em $E$, enquanto no estado $\Psi$, é $\left\langle\Psi\left|P_{E}\right| \Psi\right\rangle$, onde $P_{E}$ é a projeção espectral de $A$ em $E$;

Já discutimos, na última seção do capítulo anterior, sobre o significado da separabilidade de $\mathcal{H}$ (na subseção 4.4.2) e sobre a impossibilidade em geral de estender a definição de um operador autoadjunto ilimitado (associado a observáveis que podem assumir valores ilimitados) a todo o espaço de Hilbert, enquanto que operadores limitados, isto é, que satisfazem $\|A \psi\| \leq C\|\psi\|$, para todo $\psi \in \mathcal{H}$, em que $C$ é o que se entende por norma do operador, sempre podem ter o domínio estendido 2. A propriedade de ser limitado,

\footnotetext{
${ }^{1}$ Segundo a teoria da medida, não se pode associar uma medida a todos os subconjuntos de um dado conjunto mantendo certas propriedades como aditividade, $\mu\left[\bigcup_{n} A_{n}\right]=\sum \mu\left[A_{n}\right]$, sendo $A_{n}$ conjuntos disjuntos e a soma podendo ser infinita, apenas a alguns conjuntos particulares chamados de mensuráveis. Dada a medida que satisfaz $\mu\left[\left(a_{n}, b_{n}\right)\right]=b_{n}-a_{n}$, para o intervalo aberto $\left(a_{n}, b_{n}\right)$ existe um conjunto máximo até onde se pode estender essa medida para além dos intervalos abertos, esse é conjunto de Borel.

${ }^{2}$ Esse é o teorema de Hahn-Banach da análise funcional.
} 
para operadores lineares, pode ser demonstrada equivalente a ser contínuo.

Observe que na formulação de Von Neumann se $\Psi$ e $\Phi$ são estados, qualquer combinação linear a coeficientes complexos também o é, o que desconsidera o fato experimental conhecido como regras de superseleção, que dizem que certas superposições de estados não são fisicamente realizáveis. Mas é possível mostrar, como um teorema, que, dado qualquer coleção $M$ de vetores num espaço de Hilbert $\mathcal{H}$ tal que $\mathcal{H}=\operatorname{span}(M)$ (o conjunto de todas as combinações lineares finitas, ou infinitas convergentes), podemos decompor $\mathcal{H}$ da forma $\left.\mathcal{H}=\sum_{N}{ }^{\oplus} L_{N}\right]^{3}$ de tal modo que $L_{N}=\operatorname{span}_{N}$, onde $M_{N}=M \cap L_{N}$ é coerente e $M=\bigcup_{N} M_{N}$. Coerência significa que todas as combinações lineares finitas de elementos de $M_{N}$ estão em $M_{N}$, não sendo realizáveis as combinações lineares entre subespaços $M_{N}$ diferentes. De modo que a ideia de espaço de Hilbert de estados é válida sem restrições em cada $L_{N}$. Observáveis são então operadores autoadjuntos em $\mathcal{H}$ cujo domínio contém todos os vetores físicos $\Psi \in M$ e deixam invariantes cada um dos subespaços coerentes $M_{N}$. É possível mostrar que o conjunto de observáveis deve ser irredutível em cada subespaço $M_{N}$.

O item 3. faz uso da generalização para dimensão infinita do teorema espectral feita por Von Neumann:

Teorema 1 (Espectral). Dado um operador autoadjunto A definido no espaço de Hilbert $\mathcal{H}$, existe uma única família de projeções $E(\lambda)=E^{*}(\lambda)=E^{2}(\lambda), \lambda \in \mathbb{R}$, chamada de densidade espectral, tal que:

1. $E(\lambda)$ é monotonamente crescente, isto é, $E\left(\lambda_{1}\right) \leq E\left(\lambda_{2}\right)$, quando $\lambda_{1} \leq \lambda_{2}$, no sentido: $\left\langle\psi\left|E\left(\lambda_{1}\right)\right| \psi\right\rangle \leq\left\langle\psi\left|E\left(\lambda_{2}\right)\right| \psi\right\rangle$ para todo $\psi \in \mathcal{H}$;

2. $E(\lambda)$ é fortemente contínuo pela direita, o que se denota $s-\lim _{\lambda \rightarrow \lambda_{1}^{+}} E(\lambda)=E\left(\lambda_{1}\right)$, isto é, $\lim _{\lambda \rightarrow \lambda_{1}^{+}} E(\lambda) \psi=E\left(\lambda_{1}\right) \psi$ no sentido de convergência definido em $\mathcal{H}$;

3. $s-\lim _{\lambda \rightarrow \infty} E(\lambda)=I d$ e $s-\lim _{\lambda \rightarrow 0} E(\lambda)=0$;

\footnotetext{
${ }^{3}$ A soma direta de espaços de Hilbert $\mathcal{H}_{1}$ e $\mathcal{H}_{2}$, denotada $\mathcal{H}_{1} \oplus \mathcal{H}_{2}$, ou $\mathcal{H}=\sum_{N=1}^{2} \oplus \mathcal{H}_{N}$, é o espaço de Hilbert dos pares ordenados $\Psi=\left(\psi_{1}, \psi_{2}\right), \psi_{1} \in \mathcal{H}_{1}$ e $\psi_{2} \in \mathcal{H}_{2}$, com produto interno $\langle\Psi, \Phi\rangle=$ $\left\langle\psi_{1}, \phi_{1}\right\rangle_{1}+\left\langle\psi_{2}, \phi_{2}\right\rangle_{2}$, sendo $\Phi=\left(\phi_{1}, \phi_{2}\right)$ e $\langle *, *\rangle_{i}$ é o produto interno de $\mathcal{H}_{i}$. Analogamente, podemos definir a soma direta de $M$ espaços de Hilbert, $\mathcal{H}=\sum_{N=1}^{M} \oplus \mathcal{H}_{N}$, ou mesmo a soma de um número infinito contável $\mathcal{H}=\sum_{N=1}^{\infty} \oplus \mathcal{H}_{N}$, como o espaço de Hilbert das sequências $\left(\psi_{1}, \psi_{2}, \cdots\right), \psi_{i} \in \mathcal{H}_{i}$
} 
e

$$
A=\int \lambda d E(\lambda)
$$

A integral acima é definida primeiro para funções simples, $f(\lambda)=\sum f_{n} \chi_{B_{n}}$, uma soma finita onde $f_{n} \in \mathbb{R}, \chi_{n}$ é uma função $\mathbb{R} \rightarrow \mathbb{R}$ igual a 1 em $B_{n} \subseteq \mathbb{R}$ e 0 em caso contrário, sendo $B_{n}$ de uma das formas: $B_{n}=\left(a_{n}, b_{n}\right)$, ou $B_{n}=\left\{a_{n}\right\}$.

Definimos $\int f(\lambda) d E(\lambda)=\sum f_{n} P_{B_{n}}$, onde $P_{B_{n}}$ é um projeção construída a partir das projeções $E(\lambda): P_{B_{n}}=\lim _{\lambda \rightarrow b_{n}^{-}} E(\lambda)-E\left(a_{n}\right)$ no primeiro caso e $P_{\left\{a_{n}\right\}}=E\left(a_{n}\right)-$ $\lim _{\lambda \rightarrow a_{n}^{-}} E(\lambda)$ no segundo. Para uma função geral, $\int \phi(\lambda) d E(\lambda)$ é definida por uma sequência de funções simples $\phi_{n}(\lambda) \leq \phi(\lambda)$ que converge pontualmente, ou seja $\phi_{n}(x) \rightarrow \phi(x)$ para todo $x$, para $\phi$ :

$$
\int \phi(\lambda) d E(\lambda)=s-\lim _{n \rightarrow \infty} \int \phi_{n}(\lambda) d E(\lambda),
$$

sendo o domínio desse operador, o conjunto dos $\psi \in \mathcal{H}$ tal que a seguinte integral de Stieltjes ${ }^{4}$ é finita:

$$
\int|\phi(\lambda)|^{2} d(\psi, E(\lambda) \psi) \leq \infty
$$

Observe que a integral acima é o produto interno $\left(\int \phi(\lambda) d E(\lambda) \Psi, \int \phi(\lambda) d E(\lambda) \Psi\right)$ quando aplicamos a definição da integral e as propriedades de $E(\lambda)$ (primeiramente para funções simples).

A integral de Stieltjes é a base para a representação dos operadores no espaço de Hilbert das funções de quadrado (Lebesgue) integrável. É um conceito intermediário entre a integral de Riemann e mais geral integral de Lebesgue, sendo um caso particular dessa última. Em particular, é possível, a partir dela, definir a transformação unitária que

\footnotetext{
${ }^{4}$ A integral de Stieltjes é definida de maneira totalmente análoga, primeiramente para funções simples, cuja integral é da forma $\int f(\lambda) d \rho(\lambda)=\sum f_{n} \mu\left(B_{n}\right)$, sendo $\rho$ uma função de $\lambda$ monotonamente crescente e contínua pela direita. Observe que se $\rho(\lambda)=(\Psi, E(\lambda) \Psi)$, a propriedade 3 implica $\lim _{\lambda \rightarrow \infty} \rho(\lambda)=1$ e $\lim _{\lambda \rightarrow-\infty} \rho(\lambda)=0 . \mu\left(B_{n}\right)$ é a medida de $B_{n}$ e $\mu\left[\left(a_{n}, b_{n}\right)\right]=\left(\lim _{\lambda \rightarrow b_{n}^{-}} \rho(\lambda)-\rho\left(a_{n}\right)\right)$ e $\mu\left[\left\{a_{n}\right\}\right]=\rho\left(a_{n}\right)-$ $\lim _{\lambda \rightarrow a_{n}^{-}} \rho(\lambda)$. A integral de uma função $\phi(\lambda): \mathbb{R} \rightarrow \mathbb{R}^{+}$, positiva definida, é dada por $\int \phi(\lambda) d \rho(\lambda)=$ $\sup _{\phi_{n} \leq \phi} \int \phi_{n}(\lambda) d \rho(\lambda)$. Para uma função geral $\phi: \mathbb{R} \rightarrow \mathbb{R}^{+}$, decompomos a mesma como $\phi=\phi^{+}-\phi^{-}$, sendo $\phi^{ \pm}$positiva definida e $\int \phi(\lambda) d \rho(\lambda)=\int \phi^{+}(\lambda) d \rho(\lambda)-\int \phi^{-}(\lambda) d \rho(\lambda)$.
} 
diagonaliza um operador autoadjuntt ${ }^{5}$. Assim sendo,

$$
P_{E}=\int \chi_{E} d E
$$

em que $E$ ser um conjunto Borel significa que $\chi_{E}$ é integrável no sentido definido pela teoria da medida e integração.

O teorema espectral tem três versões: uma para operadores autoadjuntos, uma para operadores normais (que comutam com a sua adjunta) e uma para operadores unitários. Um caso particular é quando o espectro do operador é discreto, ou seja, existe um conjunto ortonormal completo $\Psi_{n}, n=1,2 \cdots$ de autoestados de $A$, com autovalores $\lambda_{1}, \lambda_{2}, \ldots$ Para estes:

$$
E(\lambda)=\sum_{\lambda_{n} \leq \lambda} E_{\lambda_{n}}
$$

em que $E_{\lambda_{n}}$ são projeções mutuamente ortogonais dispostas em ordem crescente com respeito ao autovalor associado.

A regra 5.5 é a prescrição de Von Neumann para definir observáveis quânticas associadas a funções clássicas do momento e posição independentemente, isto é, $f\left(q_{n}\right)$ ou $f\left(p_{n}\right)$, ou funções de operadores autoadjuntos em geral:

$$
f(A)=\int f(\lambda) d E_{A}(\lambda)
$$

enquanto a definição de $f\left(q_{n}, p_{n}\right)$, ou mais geralmente, a função de operadores que não comutam, é ambígua, conforme o já argumentado.

É possível mostrar que, quando o operador $A$ é limitado e $f(x):[a, b] \rightarrow \mathbb{R}$, onde $b=$ $\sup _{\psi \in \mathcal{H},\|\psi\|=1}(\psi, A \psi)$ e $a=\inf _{\psi \in \mathcal{H},\|\psi\|=1}(\psi, A \psi)^{6}$, sendo $f$ contínua, existe uma sequência

\footnotetext{
5 Em particular, um operador autoadjunto $A$ pode ser representado como o operador multiplicação $x$ nos espaço de Hilbert $\mathcal{L}^{2}(\mathbb{R})$ das funções $f(x)$ se, e somente se, o espectro de $A$, conjunto dos $\lambda$ tais que $(A-\lambda)$ é não inversível, é $\mathbb{R}$ e $A$ é cíclico, ou seja, $e^{i \lambda A} f$ é denso em $\mathcal{H}$ para algum $f \in \mathcal{H}$, ou seja, gera um subespaço cujas combinações lineares aproximam arbitrariamente qualquer elemento de $\mathcal{H}$, o que equivale a $P_{[a, b)} f$ ser denso.

${ }^{6} \sup A$ denota o supremo do subconjunto $A$ dos reais, que é um valor real definido pelas seguintes propriedades: $\sup A \geq x$ para todo $x \in A$ e dado qualquer $y$ qua satisfaça essa primeira condição, temos que $\sup A \leq y$. O ínfimo, $\inf A$, é definido de maneira análoga revertendo as desigualdades. Um conjunto limitado superior/inferiormente não necessariamente possui um máximo/mínimo, mas possui um supremo/ínfimo.
} 
de polinômios de grau finito que convergem uniformemente $7^{7}$ para $f(x)$ no seu domínio (ainda que $f$ não seja analítica, esse é o teorema de Weierstrass) e $f(A)$ é justamente o operador obtido aplicando-se esses polinômios a $A$, dando origem a uma série de operadores que convergem no sentido forte, isto é, $\left\|P_{n}(A)-P_{n+m}(A)\right\| \rightarrow 0$, com a norma de operador previamente definida. A construção desses polinômios é justamente a base da prova do teorema espectral de Von Neumann, que para operadores limitados é o operador correspondente a $\chi_{[a, \lambda)}$. No caso de operadores autoadjuntos ilimitados, o teorema primeiro é demonstrado para operadores unitários, segundo o qual um operador unitário pode ser decomposto da seguinte forma:

$$
\int_{0}^{2 \pi} e^{i \lambda} d E(\lambda)
$$

em que $E(t)=0$ para todo $t \leq 0$ e $E(1)=I 8$ para $t \geq 1$, onde a demonstração é semelhante ao caso de operadores limitados, exceto que para aproximar $\chi_{[a, \lambda)}$, usa-se uma aproximação por polinômios em $e^{i x}$ da forma:

$$
p\left(e^{i x}\right)=\sum_{k=-n}^{n} c_{k} e^{i k x},
$$

que são então mapeados num operador segundo a regra: $p\left(e^{i x}\right)=p(U)$. A versão para operadores limitados é obtida pela correspondência entre operadores unitários e autoadjuntos conhecida como transformada de Caylay, em que um operador autoadjunto A é mapeado num operador unitário $k(A)$ :

$$
k(A)=\frac{A-i}{A+i} .
$$

Contrariamente à prescrição física usual, definir a uma função de um operador autoadjunto por uma série de potências (uniformemente convergente) só é justificado quando o operador é limitado, o que exclui a maior parte dos operadores utilizados em física (mas existe outro sentido de convergência em que a série de potências pode ser reconsiderada). O fato de que o teorema espectral de Von Neumann é uma das bases da teoria de representação de operadores (e grupos) no espaço de Hilbert justifica nos delongarmos sobre ele.

Um caso particular de (3.) é quando a observável retorna 1 quando o sistema se encontra no estado $\Psi$ e 0 num estado ortogonal. Essa é a descrição matemática de um

\footnotetext{
${ }^{7}$ Isto é, $\sup _{x}\left|p_{n}(x)-f(x)\right| \rightarrow 0$

8 No que segue, poderemos denotar a identidade por $I$ ou $\epsilon$, para o caso de grupos de Lie.
} 
aparato que objetiva determinar se um dado sistema de estudo se encontra no estado $\Psi$. Nesse caso, a decomposição espectral é trivial:

$$
A=|\Psi\rangle\langle\Psi|
$$

e a probabilidade de detecção do sistema no estado $\Psi$, isto é, da medida de $A$ retornar 1 , estando inicialmente o mesmo no estado $\Phi$, é dado pelo produto de raios:

$$
[\Phi, \Psi]=|(\Psi, \Phi)|^{2}
$$

onde $($,$) denota o produto interno em \mathcal{H}$ entre quaisquer elementos representativos dos respectivos raios.

Uma vez que, no contexto clássico, uma simetria é uma transformação no espaço de parâmetros, isto é, uma mudança na forma de descrever o sistema, que não afeta as equações que governam a dinâmica, podemos estender essa definição para a descrição quântica. Considerando que, classicamente, um sistema estar no instante $t$ no estado $\left(q_{n}, p_{n}\right)$ implica que qualquer medida de $f\left(q_{n}, p_{n}\right)$ irá produzir sempre o mesmo resultado, o que não se verifica no contexto quântico, segue que a definição de simetria deve, além de considerar a dinâmica, considerar as previsões da teoria no instante $t$ segundo o axioma (3.), a previsão sobre a probabilidade de um particular resultado de medida realizado num sistema inicialmente no estado $\Phi$. Ou seja, a mudança de descrição, uma transformação $\hat{T}$ de raios em raios, se aplicada no estado $\Phi$ e na da descrição de $\Psi$, o qual define o instrumento de medida, não afeta as previsões da teoria:

$$
[\hat{T} \Psi, \hat{T} \Phi]=[\Psi, \Phi]
$$

Trabalhar com o espaço de Hilbert projetivo, o espaço dos raios, não é prático. De modo que se torna necessário estender a definição de $\hat{T}$ para todo o espaço de Hilbert e isso é feito segundo o Teorema de Wigner.

Teorema 2 (unitário/antiunitário de Wigner). Sejam $\mathcal{H}$ e $\mathcal{H}^{\prime}$ espaços de Hilbert e seja $\hat{T}$ um mapa dos raios unitários de $\mathcal{H}$ nos raios unitários de $\mathcal{H}^{\prime}$ que satisfaz:

$$
|(T \Phi, T \Psi)|=|(\Phi, \Psi)|
$$

então existe um único operador $T$, definido a menos de uma fase, que estende $\hat{T}$ e 
1. é aditivo:

$$
T\left(\Psi_{1}+\Psi_{2}\right)=T\left(\Psi_{1}\right)+T\left(\Psi_{2}\right)
$$

2. ou é unitário:

$$
(T \Phi, T \Psi)=\left(T^{*} \Phi, T^{*} \Psi\right)=(\Phi, \Psi)
$$

3. ou é antiunitário:

$$
(T \Phi, T \Psi)=\overline{(\Phi, \Psi)}=(\Psi, \Phi)
$$

Observe que, como consequência, os operadores unitários são lineares, $U(a \psi)=a U(\psi)$, sendo a complexo, enquanto que os operadores antiunitários são antilineares, ou seja, $U(a \psi)=\bar{a} U(\psi)$. Isso afeta a definição da adjunta dos operadores antilineares. A seguinte definição de adjunta faz com que tanto a expressão à esquerda quanto à direita sejam antilineares em $\Psi$ e em $\Phi:(\Phi, U \Psi)=\overline{\left(U^{*} \Phi, \Psi\right)}$. Esta definição faz que, em ambos os casos, $U^{*} U=U U^{*}=I$.

O teorema acima nos diz que, quanticamente, as simetrias devem ser realizadas como transformações lineares unitárias, ou antilineares antiunitárias. Isso não implica na existência da representação do grupo no espaço de Hilbert. De fato, uma das muitas obstruções na questão da quantização canônica é que o sistema clássico quantizado é invariante pelas transformações canônicas, que preservam os parênteses de Poisson das variáveis canônicas, mas não existe realização desse grupo no espaço de Hilbert que seja uma simetria quântica, o que implica que, a princípio, duas teorias clássicas equivalentes a menos de uma transformação canônica produzem duas teorias quânticas inequivalentes. Dada a existência da realização de um grupo no espaço dos raios, o teorema de Wigner nos diz que, no espaço de Hilbert, a regra de produto é no máximo assegurada da forma:

$$
U\left[g_{1}\right] U\left[g_{2}\right]=\omega\left(g_{1}, g_{2}\right) U\left[g_{1} \cdot g_{2}\right]
$$

onde $\omega\left(g_{1}, g_{2}\right)$ é um fase $\biguplus^{9}$ que pode ser escrita da forma $e^{i \xi\left(g_{1}, g_{2}\right)}$, sendo que a unicidade da identidade do grupo, $\epsilon$, e a associatividade da multiplicação do grupo implicam nos

\footnotetext{
${ }^{9}$ Fase esta que pode ser demonstrada independente dos estados em que atuam as transformações, a menos que existam regras de superseleção que impeçam a existência de certas combinações lineares de estado.
} 
vínculos:

$$
\begin{gathered}
\xi(\epsilon, \epsilon)=0 \\
\xi\left(g_{1}, g_{2}\right)+\xi\left(g_{1} g_{2}, g_{3}\right)=\xi\left(g_{1}, g_{2} g_{3}\right)+\xi\left(g_{2}, g_{3}\right),
\end{gathered}
$$

A princípio, não é possível, por uma mudança de fase em cada $U(g)$, remover $\omega\left(g_{1}, g_{2}\right)$, a menos que:

$$
\phi\left(g_{1}, g_{2}\right)=\alpha\left(g_{1} g_{2}\right)-\alpha\left(g_{1}\right)-\alpha\left(g_{2}\right)
$$

que pode ser removida fazendo-se

$$
U[g] \rightarrow \tilde{U}[g]=e^{-i \alpha(g)} U[g] .
$$

\subsubsection{A representação de grupos de Lie}

Esse é um bom momento para especificar adicionalmente a estrutura matemática dos grupos considerados. O tipo de grupo mais relevante em física, ao qual o grupo de Lorentz pertence, é o grupo de Lie. Um grupo de Lie é um grupo com estrutura de variedade topológica que é tal que a operação $g_{1} g_{2}^{-1}$ é contínua. Em outras palavras, a noção de "elementos próximos" está definida, existe um contínuo de elementos e se $g_{1}$ é próximo de $g_{2}$, as inversas serão também próximas, bem como se $g_{1}$ e $g_{2}$ forem próximos de $g_{1}^{\prime}$ e $g_{2}^{\prime}$, o produto destes será próximo do produto daqueles.

De particular importância é o grupo de Lie conexo, isto é, aquele em que existe um caminho contínuo ligando quaisquer dois pontos. Para este, se por um lado o conhecimento de $g_{1} g_{2}^{-1}$ (que define o produto e a inversa, ou equivalentemente suas representações coordenadas) especifica o grupo, o conhecimento do grupo numa vizinhança arbitrariamente pequena da identidade é também informação suficiente para reconstruí-lo.

De fato, dado $\sigma=\sigma_{n}$, uma curva contínua conectando a identidade $\epsilon$ ao elemento $\sigma$ e uma partição de elementos suficientemente próximos nessa curva, $\sigma_{1} \ldots \sigma_{n-1}$ :

$$
\sigma=\left(\sigma_{n} \sigma_{n-1}^{-1}\right)\left(\sigma_{n-1} \sigma_{n-2}^{-1}\right) \cdots\left(\sigma_{2} \sigma_{1}^{-1}\right) \sigma_{1}
$$

se os elementos da partição forem suficientemente próximos, o produtório acima é um produto de elementos numa vizinhança $\mathcal{N}(\epsilon)$ arbitrariamente pequena da identidade. 
Assumindo o sistema de coordenadas em $\mathcal{N}(\epsilon)$ tal que $(0,0, \ldots, 0)$ sejam as coordenadas de $\epsilon$. Se $\sigma, \tau$ e $\omega=\sigma \tau$ estão em $\mathcal{N}(\epsilon)$,

$$
\sigma=\left(s^{1}, s^{2}, \cdots, s^{r}\right), \quad \tau=\left(t^{1}, t^{2}, \cdots, t^{r}\right), \quad \omega=\left(u^{1}, u^{2}, \cdots, u^{r}\right),
$$

$\mathrm{e}$

$$
u^{i}=u^{i}(s, t), i=1,2, \cdots r
$$

Uma vez que $\epsilon \tau=\tau$ e $\sigma \epsilon=\sigma$,

$$
u^{i}=s^{i}+t^{i}+\sum_{\rho, \sigma}^{r} a_{\rho \sigma}^{i} t^{\rho} s^{\sigma}+\cdots
$$

analogamente, calculando-se $\tau \sigma$ :

$$
v^{i}=t^{i}+s^{i}+\sum_{\rho, \sigma}^{r} a_{\rho \sigma}^{i} s^{\rho} t^{\sigma}+\cdots,
$$

então a diferença $w^{i}=u^{i}-v^{i}$, medida da não comutatividade do produto, é dada por:

$$
w^{i}=\sum_{\rho, \sigma}^{r}\left(a_{\rho \sigma}^{i}-a_{\sigma \rho}^{i}\right) s^{\rho} t^{\sigma}+\cdots
$$

as quantidades

$$
c_{\rho \sigma}^{i}=a_{\rho \sigma}^{i}-a_{\sigma \rho}^{i}
$$

são chamadas de constantes de estrutura. Estas definem um tensor que é chamado de tensor de estrutura e é sempre possível introduzir uma mudança de coordenadas na vizinhança de $\epsilon$ tal que:

$$
a_{\rho \sigma}^{i}=\frac{1}{2} c_{\rho \sigma}^{i}
$$

Da associatividade do produto, decorre que:

$$
c_{\rho \sigma \tau}^{i}+c_{\sigma \tau \rho}^{i}+c_{\tau \rho \sigma}^{i}=0,
$$

onde $c_{\rho \sigma \tau}^{i}=c_{\omega \rho}^{i} c_{\sigma \tau}^{\omega}$.

Se a informação numa vizinhança arbitrária da identidade é suficiente para reconstruir o grupo e, numa vizinhança arbitrariamente pequena, os primeiros termos das séries $5 . 2 8 \longdiv { 5 . 2 9 }$ são os mais relevantes e, além disso, as constantes de estrutura são os termos de mais baixa ordem a diferenciar as transformações, somos levados a conclusão intuitiva de que as constantes de estrutura definem o grupo, dado 5.32. Esse problema foi formalmente 
resolvido por Sophus Lie, considerando a realização de grupos como difeomorfismos no próprio espaço de parâmetros do grupo, isto é, através das realizações das translações $\grave{a}$ esquerda $\tau_{\alpha}: \chi \rightarrow \alpha \chi$ no espaço de parâmetros do grupo. Lie considerou a representação da função $\Phi\left(\phi^{1}, \phi^{2}, \ldots ; \theta^{1}, \theta^{2}, \ldots\right)$ que determina o produto no espaço de parâmetros, considerou as equações diferenciais obedecidas, as quais são determinadas pelas constantes de estrutura, e as condições para a existência de solução. Estas são justamente a antissimetria de $c_{\sigma \rho}^{i}$ e a propriedade 5.33 .

Uma interpretação alternativa para as constantes de estrutura pode ser obtida através das transformações infinitesimais geradas pelo grupo $\mathcal{G}$ numa variedade $\mathfrak{G}$ em que o grupo atua como um conjunto de difeomorfismos. Dado $p \in \mathfrak{G}$ o conhecimento de $\sigma(t) p$ para toda curva em $\mathcal{G}$ e todo $p$, ou equivalentemente $\frac{d}{d t} \sigma(t) p$ para todo todo $t$ e $p$, determina a representação do grupo. Podemos definir um campo vetorial associando o vetor $\left.\frac{d}{d t} \sigma(t) p\right|_{t=t_{0}}$ a cada ponto $\sigma\left(t_{0}\right) p$ para uma dada curva fixa, o que é chamado de transformação infinitesimal. Obviamente $t_{0}$ é um ponto arbitrário e pode ser escolhido como 0, mas, além disso, não existem tantas transformações infinitesimais assim, apenas um espaço vetorial de dimensão finita. Fazendo $\mathfrak{G}=\mathcal{G}$, estas são obtidas a partir de um único vetor no espaço tangente da identidade do grupo, $\epsilon$, considerando o difeomorfismo associado ao elemento $g$, denotado $L_{g}: \chi \rightarrow g \chi$, usamos a diferencial dessa transformação para mapear o vetor em $\epsilon$ no vetor em $g$, que forma o espaço vetorial dos campos vetoriais invariantes à esquerda. Estes satisfazem (aplicamos aqui o comutador de Lie definido do capítulo 2):

$$
\left[X_{i}, X_{j}\right]=c_{i j}^{k} X_{k}
$$

onde dada a conexão entre estes campos vetoriais e $\sigma(t) p$, decorre que sua especificação também determina o grupo.

Definir um espaço vetorial onde está definido um produto antissimétrico satisfazendo a identidade de Jacobi:

$$
\begin{gathered}
{\left[X_{i}, X_{j}\right]=-\left[X_{j}, X_{i}\right]} \\
{\left[X_{i},\left[X_{j}, X_{k}\right]\right]+\left[X_{k},\left[X_{i}, X_{j}\right]\right]+\left[X_{j},\left[X_{k}, X_{i}\right]\right]=0,}
\end{gathered}
$$

possui exatamente a mesma quantidade de informação que definir um conjunto de constantes de estrutura 5.31 satisfazendo 5.33. Isto define uma álgebra de Lie, fruto da praxe 
matemática de expressar a mesma informação de um modo diferente a partir de conexões a princípio não óbvias.

Dito isso, podemos enunciar o teorema que governa a ocorrência de representações intrinsecamente projetivas 5.20 , tais que as fases não podem ser expressas da forma 5.23 . Para tal, enunciemos as condições tais que qualquer representação projetiva pode ser transformada, por uma mudança de fase 5.24, numa representação própria. Primeiramente, observe que a existência do caminho ligando $\epsilon$ a um elemento $\sigma$ arbitrário é uma propriedade topológica. A estrutura algébrica do grupo não vincula a sua estrutura topológica, ou seja, entre dois grupos pode ser estabelecida uma correspondência $\pi$, chamada de homomorfismo quando a correspondência inversa não pode ser globalmente definida, que preserva produtos e inversas, ainda que estes tenha topologias diferentes. Todo grupo de Lie conectado admite o que é chamado de grupo de cobertura universal, que é a versão (ou representação) simplesmente conectada do mesmo, ou seja, existe um caminho contínuo entre quaisquer elementos e qualquer caminho pode ser continuamente deformado em qualquer outro. A propriedade de ser simplesmente conectado é uma das que permite, de maneira independente do caminho, ajustar as fases no produto 5.20 . A outra condição é que a álgebra de Lie seja livre de cargas. Ser livre de cargas significa que, ao modificar a definição original da álgebra de Lie de modo a incluir constantes reais $C_{i j}$ tais que:

$$
\left[X_{i}, X_{j}\right]=c_{i j}^{k} X_{k}+C_{i j} I
$$

existe um conjunto constantes reais $\phi_{j}$ tais que $\bar{X}_{j}=X_{j}+\phi_{j} I$ satisfaz

$$
\left[\bar{X}_{i}, \bar{X}_{j}\right]=c_{i j}^{k} \bar{X}_{k}
$$

isto é, remove os $C_{i j}$ arbitrários.

É possível mostrar que o grupo de Lorentz tem a álgebra de Lie livre de cargas, mas não é simplesmente conectado, de modo que, para aplicar o teorema anterior, precisamos recorrer ao grupo de cobertura universal, representando este, ao invés do grupo de Lorentz propriamente dito. Para o grupo de Lorentz, o grupo de cobertura universal é $S L(2, \mathbb{C})$, das matrizes $2 \times 2$ com entradas complexas e determinante 1 . De fato, um quadrivetor $x^{\mu}$ pode ser mapeado na matriz $X$, que é hermitiana $2 \times 2$ :

$$
X=x^{\alpha} \sigma_{\alpha}=\left[\begin{array}{cc}
x^{0}+x^{3} & x^{1}-i x^{2} \\
x^{1}+i x^{2} & x^{0}-x^{3}
\end{array}\right],
$$


onde $\sigma^{0}$ é a identidade e $\sigma^{i}$ são as matrizes de Pauli. O determinante dessa matriz é a distância de Minkowski: $\operatorname{det} X=\left(x^{0}\right)^{2}-x^{2}$. Reciprocamente, $x^{\mu}=\frac{1}{2} \operatorname{Tr}\left(\sigma_{\mu} X\right)$. A transformação $X^{\prime}=A X A^{*}$, onde $A$ é qualquer matriz de entradas complexas e de determinante 1 (i.e. $A \in S L(2, \mathbb{C})$ ), preserva as propriedades de $X$, bem como seu determinante, e está associada a uma única transformação de Lorentz dada por:

$$
\Lambda_{\nu}^{\mu}=\frac{1}{2} \operatorname{Tr}\left(\sigma_{\mu} A \sigma_{\nu} A^{*}\right)
$$

A relação acima, na verdade, mapeia $S L(2, \mathbb{C})$ no subgrupo $L_{+}^{\uparrow}\left(\operatorname{det} \Lambda=1\right.$ e $\left.\Lambda_{0}^{0} \geq 1\right)$, um dos quatro componentes conectados do grupo de Lorent 20 . De fato, $\Lambda_{0}^{0}=\frac{1}{2} \operatorname{Tr}\left(A A^{*}\right)>0$ $\mathrm{e} \operatorname{det} \Lambda=111$

Podemos ir ainda além na correspondência acima introduzindo o conceito de spinor. Spinores são elementos de um espaço vetorial complexo bidimensional, cujos elementos são denotados $\Psi_{r}(r=1,2)$, e onde está definida a seguinte representação de $S L(2, \mathbb{C})$ :

$$
\Psi_{r} \rightarrow \alpha_{r s} \Psi_{s}
$$

O conceito de spinor pode ser estendido tal qual o de tensor, reservando-se o nome spinor covariante de rank 1 para o caso acima, denotando $\Psi_{\dot{r}}$ a representação conjugada complexa, que se transforma pela regra acima fazendo-se $\alpha \rightarrow \bar{\alpha}, \Psi^{r}$ definido a representação contragradiente, que se transforma pela regra acima, mas com a matriz $\beta=\left(\alpha^{T}\right)^{-1}$ e denotando $\Psi^{\dot{r}}$ a representação contragradiente complexa conjugada, que se transforma $\operatorname{com} \alpha \rightarrow \bar{\beta}$.

Definido isso, definimos spinores de rank maior pelo produto tensorial destes. Decorre que os spinores totalmente simetrizados com $n$ índices com ponto e $m$ índices sem ponto (adotamos aqui a convenção de simetrização discutida no capítulo 2):

$$
\Psi_{r_{1} \cdots r_{n} \dot{s}_{1} \cdots \dot{s}_{m}}=\Psi_{\left(r_{1} \cdots r_{n} \dot{s}_{1} \cdots \dot{s}_{m}\right)},
$$

geram todas as classes de equivalência das representações irredutíveis de dimensão finita do grupo $S L(2, \mathbb{C})$ (vide discussão sobre representações adiante). A matiz 5.39 é, então, um spinor $V_{r \dot{s}}$. Quando $m+n$ é par, temos uma representação equivalente à tensorial.

\footnotetext{
10 Os outros três são: $L_{+}^{\downarrow}\left(\operatorname{det} \Lambda=1\right.$ e $\left.\Lambda_{0}^{0} \leq 1\right), L_{-}^{\uparrow}\left(\operatorname{det} \Lambda=-1\right.$ e $\left.\Lambda_{0}^{0} \geq 1\right)$ e $L_{-}^{\downarrow}(\operatorname{det} \Lambda=$ $-1 \quad$ e $\left.\quad \Lambda_{0}^{0} \leq 1\right)$.

${ }^{11}$ Nesse caso, o resultado é trivial quando escolhemos $A$ igual à identidade. Que o determinante é igual a um para todos os elementos de $S L(2, \mathbb{C})$, também decorre do fado de que este grupo é simplesmente conectado.
} 
Todo grupo de Lie conectado admite, na vizinhança da identidade, um sistema de coordenadas canônico, isto é, tal que $(0,0, \ldots, 0)$ são as coordenadas da identidade e

$$
g\left(0, \theta_{1}, \cdots, 0\right) \cdot g\left(0, \theta_{2}, \cdots, 0\right)=g\left(0, \theta_{1}+\theta_{2}, \cdots, 0\right)
$$

sendo $g$ o mapa das coordenadas nos elementos do grupo. Sendo assim, nessa vizinhança, toda realização do grupo como uma simetria quântica é linear e unitária. De fato, $g(0, \theta, \cdots, 0)=g(0, \theta / 2, \cdots, 0)^{2}$, mas o quadrado de uma transformação linear e unitária, ou antilinear e antiunitária, é linear e unitário. Como uma vizinhança arbitrariamente pequena da identidade reconstrói o grupo de Lie conexo, segundo 5.25 , e o produto de transformações lineares unitárias é linear e unitário, a representação desse assim o é.

Na vizinhança coberta por coordenadas canônicas, existem, pelo exposto, representações unitárias dos subgrupos de um parâmetros $g(0, \cdots, t, \cdots, 0)$, denotadas $\mathcal{U}(t)$, podemos assumir que $\langle\phi|\mathcal{U}(t)| \psi\rangle$ é contínuo em $t$ para todo $\phi, \psi \in \mathcal{H}$. A essa propriedade, chamamos de continuidade fraca. Esse requerimento é na verdade muito razoável. De fato, foi provado por Von Neumann que essa propriedade é equivalente a $\langle\phi|\mathcal{U}(t)| \psi\rangle$ ser uma função mensuráve ${ }^{12}$, tendo sido provado por Lebesgue que praticamente todas as funções são mensuráveis e que a existência de funções não mensuráveis é condicionada a aceitação de certos axiomas. Mais ainda, $\mathcal{U}(t)$ ser fracamente contínuo é equivalente a condição mais forte de $\mathcal{U}(t)$ ser fortemente contínuo.

Sob essas condições, temos:

Teorema 3 (Stone). Toda família contínua de um parâmetro de transformações unitárias fortemente contínua pode ser escrita da forma:

$$
\mathcal{U}(t)=e^{-i A t}
$$

onde $A$ é um operador autoadjunto.

Ou seja, a propriedade $U\left(t+t^{\prime}\right)=U(t) U\left(t^{\prime}\right), U(t)$ unitário e fortemente contínuo, implica que podemos definir um operador autoadjunto $A$ associado.

No teorema acima, $e^{-i A t}$ é dado pela prescrição de Von Neumann e o operador $A$ é chamado de gerador da simetria. A prova do teorema de Stone passa pela demonstração da importante regra de quantização do momento em teoria quântica sob condições

\footnotetext{
${ }^{12}$ Uma função mensurável é aquela que pode ser reescrita da forma $\sum_{E} c_{E} \chi_{E}$, sendo $c_{E}$ uma constante, $E$ um conjunto mensurável e a soma sobre uma família enumerável de conjuntos.
} 
de contorno periódicas no espaço, o que será importante quando tentarmos assegurar a validade dessa regra sob hipótese de espaço-tempo não comutativo. De fato, o teorema primeiro é provado para $U_{1}(t)$ periódico de período $2 \pi$ (ou seja $U_{1}(t)=U_{1}(t+2 \pi)$, mas o período poderia ter sido escolhido como $L$ levando a um raciocínio análogo).

O teorema consiste em construir a representação espectral para $U_{1}(t)$, mostrando que

$$
U_{1}(t)=\sum_{n} e^{i n t} \int_{0}^{2 \pi} e^{-i n x} U(x) d x=\sum_{n} e^{i n t} E_{n},
$$

onde $E_{n}$ são projeções mutuamente ortogonais e:

$$
s-\lim \sum_{n} E_{n}=1
$$

o que implica na quantização do momento, pois $U(t)=e^{i P t}$, onde

$$
P=\sum_{n} n E_{n}
$$

O resultado geral é obtido fazendo $U_{1}(t)=U(t) U(2 \pi)^{-\frac{t}{2 \pi}}$ que é usado para construir uma representação espectral para $U(t)$ a partir do fato de que, pelo teorema espectral, existe uma representação para $U(2 \pi)$. O teorema de Stone nos permite associar simetrias a observáveis. Possivelmente, todas as observáveis em física quântica surjam dessa maneira, por considerações de simetria.

Neste contexto, podemos dar outro significado para as constantes de estrutura 5.31 .

$$
\left[H_{\alpha}, H_{\beta}\right]=i c_{\alpha \beta}^{\rho} H_{\rho}
$$

em que $H_{\alpha}$ é o gerador do $\alpha$-ésimo subgrupo de um parâmetro do sistema canônico de coordenadas na vizinhança da origem do grupo de Lie. Observe que 5.48 assume que todos os operadores autoadjuntos $H_{\alpha}$ podem ser definido num domínio $\Omega \subset \mathcal{H}$ comum invariante pela ação dos mesmos, ou seja $H_{\alpha} \Omega \subseteq \Omega$.

\subsubsection{A representação do Grupo de Poincaré}

Para o grupo de Poincaré, estes subgrupos são os de translação temporal, cujo gerador $H$ é a Hamiltoniana, também denotada $P^{0}$, que define o operador associado à energia; o subgrupo de translação na i-ésima direção, cujo gerador $P^{i}$ é o momento linear na iésima direção; as rotação pelo ângulo $\theta$ em torno das três direções ortogonais $x, y$ e $z$, 
cujos geradores são os componentes do momento angular $\left\{M^{23}, M^{31} \cdot M^{12}\right\}$ e os boots, transformações de Lorentz associadas a um observador que se move com velocidade $v$. Podemos construir estas últimas através de rotação de Wick $\left(x^{0}, \vec{x}\right) \rightarrow\left(\bar{x}^{0}, \vec{x}\right)=\left(i x^{0}, \vec{x}\right)$, que torna a métrica euclidiana nas coordenadas $\bar{x}^{\mu}$, a qual é preservada por rotações. Uma rotação por um ângulo $\theta$ envolvendo as coordenadas $\left(\bar{x}^{0}, \bar{x}^{i}\right)$, seguida da rotação de Wick inversa, nos leva a uma transformação linear a coeficientes complexos, a menos que usemos ângulos complexos $i \theta$. Associados a este subgrupo, temos o vetor de boost $\left\{M^{01}, M^{02}, M^{03}\right\}$. Podemos definir cada um dos subgrupos anteriores de modo alternativo, devido ao fato de qualquer transformação de Lorentz $\Lambda$ poder ser escrita da forma $e^{\omega^{\mu}{ }_{\nu}}$, sendo $\omega_{\mu \nu}=\eta_{\mu \tau} \omega^{\tau}{ }_{\nu}=-\omega_{\nu \mu}$. Cada um dos subgrupos é, então, obtido fazendo apenas uma das entradas independentes de $\omega_{\mu \nu}$ (acima da diagonal) igual ao parâmetro do subgrupo. Isso justifica a notação empregada que define um tensor antissimétrico $M^{\mu \nu}$, chamado de tensor de momento angular, tal que $U(\Lambda)=e^{i \omega_{\mu \nu} M^{\mu \nu}}$, para o qual temos a seguinte álgebra de Lie:

$$
\begin{gathered}
{\left[P^{\mu}, P^{\nu}\right]=0} \\
{\left[M^{\mu \nu}, P^{\lambda}\right]=i\left(g^{\mu \nu} P^{\lambda}-g^{\lambda \nu} P^{\mu}\right)} \\
{\left[M^{\mu \nu}, M^{\rho \sigma}\right]=-i\left(g^{\mu \rho} M^{\nu \rho}-g^{\nu \rho} M^{\mu \sigma}+g^{\nu \sigma} M^{\mu \rho}-g^{\mu \sigma} M^{\nu \rho}\right)}
\end{gathered}
$$

Ao construir representações de grupos como transformações lineares, as primeiras questões relevantes são: Como diferenciar tais representações? Quais as mais simples? Quantas representações diferentes existem? Qual a mais geral possível?

A primeira observação é sobre a existência de uma representação trivial que aplica todos os elementos do grupo no operador identidade $I$ no espaço de Hilbert. Esta é uma representação válida satisfazendo 5.1, 5.2 e 5.3, mais geralmente podemos ter uma representação $\pi$ tal que $\pi^{-1}(I)$, chamada de kernel da representação, é um subgrupo não trivial de $\mathcal{G}$, isto é, é constituído de mais que um elemento. Para excluir essa possibilidade, incluímos o conceito de representação fiel, cujo o kernel é constituído da identidade de $\mathcal{G}, \epsilon$, apenas.

Outra questão é como diferenciar as representações. Uma solução trivial é que $\pi_{1}\left(g_{1}\right) \neq$ $\pi_{2}\left(g_{1}\right)$ para pelo menos algum $g_{1}$ e consequentemente todos. Uma rápida análise nos faz pensar que esse conceito não é apropriado aos nossos propósitos. De fato, temos a 
liberdade de escolher a descrição do sistema e o estado associado a $\Psi \in \mathcal{H}$ em uma dada descrição corresponde a $U \Psi$ em outra descrição, onde $U$ é uma simetria. Isso nos leva a identificar $\pi(g), g \in \mathcal{G}$ com $U \pi(g) U^{-1}$, visto que descrevem o mesmo sistema. Duas representações $\pi_{1}$ e $\pi_{2}$ que não admitem a existência de nenhuma transformação inversível $S$, não necessariamente uma simetria, tal que $\pi_{1}(g)=S \pi_{2}(g) S^{-1}$ para todo $g \in \mathcal{G}$ são chamadas de inequivalentes e do contrário, como se poderia esperar, equivalentes.

Uma vez que tratamos apenas transformações lineares, um importante processo de simplificação pode ser introduzido. Suponhamos que exista um subespaço de $\mathcal{H}$, denotado $\mathcal{S}$, tal que $\pi(g) \mathcal{S} \subseteq \mathcal{S}$ para todo $g \in \mathcal{G}$. Então $P \pi(g) P$, sendo $P$ uma projeção no subespaço invariante, fornece uma representação que necessita menos informação para ser definida que a original ${ }^{13}$. A representação $P \pi(g) P$ é chamada de $\boldsymbol{r e d u c ̧ a ̃ o ~ d a ~ r e p r e s e n t a c ̧ a ̃ o ~} \pi$. Uma representação que não possa ser simplificada dessa maneira é chamada de irredutivel.

Dada uma representação irredutível $\pi_{1}$ no espaço vetorial $V$, podemos definir uma segunda que é inequivalente a esta, a qual denotamos por $\pi \oplus \pi$, que chamamos de soma direta das representações, e atua no espaço vetorial dos pares $\left(v_{1}, v_{2}\right)$ da forma $\left(\pi(g) v_{1}, \pi(g) v_{2}\right), v_{1}, v_{2} \in V$. Por tanto, existem representações inequivalentes. A demonstração da inequivalência é apenas uma questão de mostrar que esta última é redutível. Quando a todo subespaço invariante $W$ temos um complemento ortogonal $W^{\perp}$ também invariante, dizemos que a representação é completamente redutível.

Agora, enunciemos o primeiro resultado na direção da forma mais geral de uma representação, a qual se aplica nas representações por transformações unitárias, que representam grupos de Lie conexos.

Teorema 4. Toda representação unitária de um grupo é irredutivel ou completamente redutivel.

As transformações unitárias são fundamentais não apenas em teoria quântica, mas na teoria de grupos em si. Elas governam toda teoria de representação de grupos com um número finito de elementos e grupos de Lie compactos 14 , uma vez que todas essas

\footnotetext{
${ }^{13}$ No caso de uma representação em dimensão finita, $P \pi(g) P$ é representado por matrizes de rank menor.

${ }^{14}$ Compacto diz respeito a noção intuitiva de tamanho finito do espaço, juntamente com a noção de não ter buracos no sentido de que qualquer sequência convergente encontra um limite dentro no próprio espaço. Rigorosamente, quer dizer que dada qualquer coleção $U_{\alpha}$ de abertos tal que $\bigcup_{\alpha} U_{\alpha}=M$, sendo $M$ a variedade do grupo, podemos escolher um número finito deles cuja união ainda é igual a $M$.
} 
representações são equivalentes(no sentido discutido) a representações por transformações unitárias.

A intuição nos diz então que podemos continuar o processo de redução até decompor a representação em partes irredutíveis. Antes de enunciar a forma dessa decomposição, que nos responderá a forma mais geral da representação do grupo, enunciemos os resultados que governam a ocorrência de representações lineares irredutíveis de qualquer espécie. Enunciemos a versão melhor aplicável ao contexto analisado. Este resultado encerra a questão do significado conceitual da relação de dispersão.

Lema 1 (Shur). Seja $\pi$ uma representação unitária ou autoadjunt ${ }^{15}$ de um grupo $\mathcal{G}$ no espaço de Hilbert $\mathcal{H}, \pi$ é irredutivel se, e somente se, o comutante

$$
\pi(\mathcal{G})^{\prime} \equiv\{C \in \mathcal{B}(\mathcal{H}),[C, \pi(g)], \forall g \in \mathcal{G}\}
$$

em que $\mathcal{B}(\mathcal{H})$ é o espaço vetorial dos operadores limitados, consiste apenas dos múltiplos da identidade.

A demonstração desse teorema em dimensão infinita passa pelo teorema espectral de Von Neumann. A questão fundamental é que se $C \in \pi(\mathcal{G})^{\prime}$, então, $C^{*} \in \pi(\mathcal{G})^{\prime}$ e podemos escolher $C$ autoadjunto $\left(C \rightarrow C+C^{*}\right)$. Então, as projeções espectrais $P_{E}^{C}$, para todo $E$ Borel, definem subespaços invariantes pela ação da representação em $\mathcal{H}$ e que podem ser utilizadas para reduzir a representação. Operadores limitados evitam, como argumentado, problemas com definição do domínio. Buscamos $C$ definido em termos dos operadores já definidos, através de operações definidas para operadores no espaço de Hilbert, tais como produtos e combinações lineares (desde que os domínios sejam os mesmos e invariantes pela ação dos operadores), isso representa uma extensão do conceito de álgebra de Lie chamada de álgebra universal envelopante $\mathcal{U}(\mathfrak{g})$, da álgebra de Lie $\mathfrak{g}$.

No caso da representação de $P_{+}^{\uparrow}, L_{+}^{\uparrow}$ incluindo translações temporais e espaciais, este é gerado por $e^{-i \lambda H}, e^{-i \lambda_{i} P^{i}}$ e $e^{-i \lambda_{\mu \nu} M^{\mu \nu}}, \lambda$ real. Suponha $f \in \mathcal{U}(\mathfrak{g})$ autoadjunto, tal que $\left[f, H_{\alpha}\right]=0$, então $e^{-i f} \in \pi(\mathcal{G})^{\prime}$. Cada um dos $f$ é chamado de Casimir. Para $P_{+}^{\uparrow}$ temos

${ }^{15}$ No sentido de que para cada $g \in \mathcal{G}$ existe $g^{\prime} \in \mathcal{G}$ tal que $\pi\left(g^{\prime}\right)=\pi^{*}(g)$ 
o seguinte conjunto de operadores de Casimir algebricamente independentes.

$$
\begin{gathered}
P^{2}=H^{2}-\vec{P}^{2} \\
W=-w^{2}, \operatorname{com} w_{\rho}=\frac{1}{2} \epsilon_{\alpha \mu \nu \rho} P^{\alpha} M^{\mu \nu}, \\
\operatorname{sign}\left(P^{0}\right)=\theta\left(P^{2}\right) \epsilon\left(P^{0}\right),
\end{gathered}
$$

em que

$$
\left[P^{\mu}, w^{\nu}\right]=0 .
$$

sign é o sinal da energia, que pode ser transformado num operador autoadjunto $\operatorname{sign} P^{0} \Psi=$ $\Psi$ se $\Psi$ é uma superposição de autoestados de energia associados a autovalores positivos e tais que $P^{2}$ seja positivo. O Casimir gerado pelo quadrimomento $P^{\mu}$ é a chamada relação de dispersãa e se traduz numa equação entre autovalores da energia e do momento em representações irredutíveis. $w^{\rho}$ é o chamado vetor de Pauli-Lubanski-Bargmann. $\theta(t)$ é a função degrau que é 0 para $t<0$ e 1 do contrário. $\epsilon$ é um sinal $(+1,-1), \theta\left(P^{2}\right)$ e $\epsilon\left(P^{0}\right)$ são definidos pela prescrição de Von Neumann.

O Lema de Shur nos diz que o número de representações unitárias irredutíveis inequivalentes de $P_{+}^{\uparrow}$ é o mesmo que o de conjuntos ordenados de possíveis autovalores reais dos operadores de Casimir independentes $P^{2}, W$ e $\operatorname{sign}\left(P^{0}\right)$ : denotemos essas representações $\mathcal{U}_{\left(m^{2}, w, \epsilon\right)}$. Na física, existe um postulado que restringe a ocorrência dessas representações em sistemas quânticos relativísticos. Este diz que apenas representações satisfazendo $m^{2} \geq 0$ e $\epsilon=1$ ocorrem na natureza. O que equivale a dizer que:

Postulado 1 (Condição espectral). O espectro do operador energia-momento $P^{\mu}$ das representações irredutiveis (e, por conseguinte, das mais gerais) de $S L(2, \mathbb{C})$ pertence ao fechamento do cone de luz do futuro $\bar{V}^{+}$.

Ou seja, a energia é positiva definida, condição ligada à estabilidade da matéria. Destas representações, as que diferem no sinal da energia são adjuntas entre si (os operadores correspondentes nas duas representações são adjuntos uns dos outros). No caso $m^{2}=0$, $P^{\mu} \neq 0$, temos duas possibilidades para $W$ : na primeira, $W=0$, que implica $w^{\mu}=\lambda P^{\mu}, \lambda$ é um inteiro ou semi-inteiro chamado de helicidade. Quando $W=-w^{2}=\rho^{2}, \rho>0$ temos a chamada representação contínua de spin, que é assumida não ocorrer na natureza.

Passemos agora ao problema de construir as representações irredutíveis do grupo de Poincaré. Ao buscar representações de um conjunto de operadores autoadjuntos (com um 
domínio comum deixado invariante pela aplicação dos operadores), ou mais geralmente operadores normais, que comutam com a sua adjunta, podemos sempre começar de um conjunto (maximal) algebricamente independente de operadores que comutam, pois todas as representações de uma álgebra desse tipo podem ser colocadas, via transformações unitárias, numa forma padronizada (veja ainda o teorema Gelfand-Naimark e a construção GNS no capítulo seguinte). Considerando 5.49, esse conjunto contém apenas o quadrimomento $P^{\mu}$. Pelo exposto, esta representação será irredutível se os operadores $\left(P^{2}, W, \operatorname{sign}\left(P^{0}\right)\right)$ forem múltiplos da identidade nessa representação.

A representação da subálgebra gerada por $P^{\mu}$ começa pela identificação da base comum de autoestados generalizados. Um autoestado generalizado $\Psi_{\lambda}$ associado ao autovalor $\lambda$ é uma classe de equivalência de sequências de estados mormalizados $\Psi_{n}$ tais que:

$$
(H-\lambda) \Psi_{n} \rightarrow 0
$$

Duas sequências $\Psi_{n}$ e $\Phi_{n}$ tais que $\left\|\Psi_{n}-\Phi_{n}\right\| \rightarrow 0$ e $\left\|(H-\lambda)\left(\Psi_{n}-\Phi_{n}\right)\right\| \rightarrow 0$ são consideradas equivalentes e definem a mesma classe $\Psi=\left\{\Psi_{n}\right\}$. Operadores autoadjuntos são fechados (têm o gráfico fechado, segundo definição do footnote 10 do capítulo anterior), para estes operadores, quando $\left\|\Psi_{n}-\Phi_{n}\right\| \rightarrow 0$, decorre que $(H-\lambda)\left(\Psi_{n}-\Phi_{n}\right) \rightarrow 0$, ou não é uma sequência convergente (uma propriedade que pode ser mostrada equivalente a ser fechado). O conjunto de todos os $\lambda$ para os quais existe uma sequência $\Psi_{n}$ não convergindo para zero e satisfazendo 5.55 forma o espectro de $H$, denotado $\sigma(H)$. Quando tais sequências são convergentes, dizemos que o autovalor é discreto, do contrário, contínuo. Denotamos o conjunto dos autovalores discretos de $H$ por $\sigma_{d}(H)$ e o conjunto dos autovalores contínuos de $H, \sigma_{c}(H)=\sigma(H)-\sigma_{d}(H)$. Uma definição de $\sigma(H)$ que pode ser mostrada equivalente a essa é o conjunto dos $\lambda$ tais que $(H-\lambda)^{-1}$ não existe.

Uma caracterização alternativa para os autoestados generalizados, mais conveniente por permitir reconstruir o espaço de Hilbert a partir dos autoestados generalizados de um operador autoadjunto, seria através da correspondência entre vetores e funcionais lineares contínuos (conceito equivalente a ser limitado) no espaço de Hilbert $\mathcal{H}$. Existe um teorema, devido a Riesz, que diz que todo funcional linear contínuo em $\mathcal{H}$, cujo conjunto forma o chamado espaço dual $\mathcal{H}^{*}$, é da forma $(\Psi, \phi)$, para um dado $\Psi$ associado ao funcional e todo $\phi \in \mathcal{H}$. Para um operador autoadjunto $A$ (de espectro discreto $\sigma_{d}(A)$ e no espaço de 
Hilbert separável), os funcionais limitados que satisfazem:

$$
\left(\Psi_{\lambda}, A \phi\right)=\lambda\left(\Psi_{\lambda}, \phi\right), \quad \text { para todo } \phi \in \mathcal{H}
$$

que formam espaços vetoriais para um mesmo $\lambda$, os autoespaços, são tais que qualquer outro funcional limitado pode ser escrito da forma:

$$
(\Psi, *)=\sum_{\lambda \in \sigma_{d}(A)} c_{\lambda}\left(\Psi_{\lambda}, *\right), \quad \sum_{\lambda \in \sigma_{d}(A)} c_{\lambda}^{2}<\infty
$$

Observe que não estamos assumindo que os autoespaços associados ao autovalor $\lambda$ são unidimensionais.

Ocorre que nem sempre existe um funcional linear limitado que satisfaz 5.56, embora $\lambda$ pertença ao espectro de $A$. Mas podemos definir autoestados generalizados associados a funcionais lineares não limitados, isto é, não contínuos em $\mathcal{H}$, não tendo, por tanto, nenhum vetor $\Psi \in \mathcal{H}$ associado, mas que ainda assim denotaremos $\left(\Psi_{\lambda}, *\right)$, ou $\Psi_{\lambda}(*)$ e que satisfazem 5.56. Para estes, 5.57 assume a forma:

$$
(\Psi, *)=\int_{\lambda \in \sigma(A)} d \mu \overline{f_{\Psi_{\lambda}}} \Psi_{\lambda}(*),
$$

onde $f_{\Psi_{\lambda}}$ é um número complexo e $d \mu$ é uma medida de integração no conjunto de autoestados generalizados (não no espectro $\sigma(A)$, o que dá conta do fato de que os autoespaços generalizados podem ser multidimensionais).

Observe que os autoestados generalizados geram uma representação funcional para o espaço de Hilbert $\mathcal{H}$ via 5.58. Ou seja, o conjunto de autoestados generalizados pode ser entendido como um conjunto de pontos de um espaço $X$ onde está definida uma medida de integração e cada $\Phi \in \mathcal{H}$ é mapeado numa função $\phi_{\lambda}$, que assume o valor $\Psi_{\lambda}(\Phi)$ no ponto $\Psi_{\lambda}$, sendo:

$$
(\Psi, \Phi)=\int d \mu \overline{\Psi_{\lambda}(\Psi)} \Psi_{\lambda}(\Phi)
$$

Nessa representação: $A \Phi$ é representado pela função $\phi^{\prime}\left(\Psi_{\lambda}\right)=\lambda \Psi_{\lambda}(\Phi)$.

O conjunto de todos os funcionais lineares em $\mathcal{H}$ é muito grande, para assegurar que o conjunto de funcionais que satisfazem 5.56 e permitem uma expansão do tipo 5.58 seja o menor possível, a classe de funcionais precisa ser especificada adicionalmente. Uma maneira conveniente de fazer isso é assumir que os funcionais são descontínuos em $\mathcal{H}$, mas mantendo continuidade em algum outro sentido (o que tem a vantagem de definir todo um 
novo conjunto de funcionais pelo próprio requerimento de continuidade modificado). Para tanto, temos que nos restringir a um subespaço $\Omega \subset \mathcal{H}$, onde está definida uma nova noção de convergência menos geral do que a de $\mathcal{H}$, mas em relação a qual $\Omega$ é completo, ou seja, nesse sentido, toda sequência convergente em $\Omega$ converge para um elemento de $\Omega$, mas neste existem sequências que convergem segundo o sentido definido em $\mathcal{H}$ e não pertencem a $\Omega$. Essa estrutura faz com que todo funcional contínuo de $\mathcal{H}$ seja um funcional contínuo de $\Omega$, cujo conjunto forma o espaço dual $\Omega^{*}$, mas não vice versa, ou seja:

$$
\Omega \subset \mathcal{H} \subset \Omega^{*}
$$

onde $\Omega$ e $\mathcal{H}$ são identificados com funcionais segundo o teorema de Riesz. Os operadors autoadjuntos que mapeiam $\Omega$ em $\Omega$ possuem autoestados generalizados $\Psi_{\lambda} \in \Omega^{*}$ definidos via:

$$
\left(\Psi_{\lambda}, A \phi\right)=\lambda\left(\Psi_{\lambda}, \phi\right), \quad \phi \in \Omega
$$

desde que a noção de continuidade de $\Omega$, que define os funcionais em questão, seja escolhida apropriadamente. Além disso, $\Omega$ pode ser escolhido denso em $\mathcal{H}$, ou seja, cada elemento de $\mathcal{H}$ é limite de uma sequência convergente em $\Omega$ (na topologia de $\mathcal{H}$ ).

Ocorre que o espaço $\Omega$ pode ser definido com uma importante propriedade: para qualquer representação do espaço de Hilbert $\mathcal{H}$ como funções de quadrado integrável em algum espaço $X$ onde está definida uma medida de integração $d \mu$, a qual definimos pela regra $\pi: \mathcal{H} \rightarrow L(X, d \mu)$, existe, para cada ponto $x \in X$, um funcional $F_{x} \in \Omega^{*}$ tal que:

$$
\pi(\phi)(x)=F_{x}(\phi), \quad \phi \in \Omega \subset \mathcal{H}
$$

a menos de um conjunto de medida nula. Em outras palavras, podemos gerar todas as representações funcionais de um espaço de Hilbert aplicando funcionais de $\Omega^{*}$ nos elementos de $\Omega$.

Ocorre que a boa propriedade de convergência de $\Omega$, que faz sempre existir o funcional 5.61, é, em certo sentido, ser similar ao espaço das funções teste, de modo que $\Omega^{*}$ é análogo ao espaço das funções generalizadas, ou distribuições. Uma distribuição é um funcional linear contínuo num espaço de funções teste. Existe mais de um espaço de funções teste, por exemplo, $\mathcal{S}(\mathcal{O}){ }^{16}$ é o mais usado em física. A equivalência entre $\Omega$

\footnotetext{
${ }^{16}$ Existem vários tipos de funções teste, cada uma com sua própria noção de convergência, usadas como
} 
e $S(\mathcal{O})$ é explícita quando $\mathcal{H}$ é representado funcionalmente, mas pode ser definida sem recorrer a essa representação através do conceito de espaço nuclear.

Observe que a sequência 5.55 não produz o autoestado generalizado 5.61 segundq ${ }^{17}$.

$$
\Psi_{\lambda}(\Phi)=\lim _{N \rightarrow \infty}\left(\Psi_{N}, \Phi\right)
$$

mas é possível mostrar que existe uma sequência divergente de constantes $c_{n}$ tal que cada $c_{n} \Psi_{n}$ define o operador de $\Omega^{*}$ desejado. Se $[A, B]=0$ e $\lim _{N}(A-\lambda) \Psi_{N}=0$, então $\lim _{N} B(A-\lambda) \Psi_{N}=\lim _{N}(A-\lambda) B \Psi_{N}=0$ Por tanto, a ação de $B$ deixa os autoespaços de $A$ invariantes e vice versa. Por tanto, é possível encontrar autoestados de $B$ nos autoespaços de $A$.

Numa representação redutível, $P^{0}$ e $P^{i}$ são operadores algebricamente independentes, mas tal não ocorre numa representação irredutível. Como dito, numa representação irredutível, $P^{2}$ é multiplo da identidade, só precisamos realizar $P^{i}, i=1,2,3$, e $P^{0}$ já estará determinado. Denotemos, $\Psi_{p^{\mu}}$ o conjunto de autoestados generalizados comuns a $P^{i}$, decorre que:

$$
P^{2} \int d \mu f_{\Psi_{p^{\mu}}} \Psi_{p^{\mu}}=m^{2} \int d \mu f_{\Psi_{p^{\mu}}} \Psi_{p^{\mu}}
$$

o que nos leva a interpretar a representação irredutível $\mathcal{H}_{\left(m^{2}, w, \epsilon\right)}$ como o espaço de Hilbert de uma única partícula de massa $m$. Para seguir adiante no problema da representação, precisamos determinar o espectro dos operadores $P^{\mu}$. Para tanto, observe que:

$$
U(\Lambda, a) P^{\rho} U^{-1}(\Lambda, a)=\Lambda_{\mu}{ }^{\rho} P^{\mu}
$$

Assim sendo, dado qualquer $\Psi=\Psi_{N}$, como em 5.55, tal que $\lim _{N \rightarrow \infty}\left(P^{\mu}-k^{\mu}\right) \Psi_{N}=0$, passo intermediário na definição de funções generalizadas como a delta de Dirac na física a partir da sua noção de continuidade. A mais usada são as de rápido decrescimento, $\mathcal{S}(\mathcal{O})$, onde $\mathcal{O} \subset \mathbb{R}^{n}$ é o conjunto onde as funções estão definidas. Estas são as $f(x) \in \mathcal{C}^{\infty}$ tais que ||$f(x) \|_{N}=\sup _{n+m \leq M}\left|x^{n} \partial^{m} f(x)\right|<\infty$, $n, m \geq 0$. Uma sequência de funções $f_{n}(x)$ é convergente para 0 se $\left\|f_{n}(x)\right\|_{N} \rightarrow 0$ para todo $N$. Um funcional linear $F$ é contínuo se $F\left(f_{n}(x)\right) \rightarrow 0$ quando $f_{n}(x) \rightarrow 0$.

${ }^{17}$ De fato, $\left(\phi,(H-\lambda) \Psi_{n}\right)=\left((H-\lambda) \phi, \Psi_{n}\right) \rightarrow 0$, mas se $\lambda$ é um autovalor contínuo de $\mathcal{H}$, decorre que $R(H-\lambda)^{-}=\mathcal{H}$, mas $R(H-\lambda) \neq \mathcal{H}$, onde $R$ é a imagem $(H-\lambda)$. Se $\left\|\Psi_{n}\right\|=1$ para todo $n$, $\left|\lim _{n}\left(\Psi_{n}, \phi\right)\right| \leq\|\phi\|$, logo, define um operador contínuo e igual a zero em $R(H-\lambda)$, um conjunto denso em $\mathcal{H}$, por tanto, esse limite é igual a zero em todo $\mathcal{H}$. 
temos que:

$$
\begin{gathered}
\lim _{N \rightarrow \infty}\left(\Lambda_{\rho}^{-1 \mu} U(\Lambda, a) P^{\rho} U^{-1}(\Lambda, a)-U(\Lambda, a) k^{\mu} U^{-1}(\Lambda, a)\right) \Psi_{N}=0 \\
\lim _{N \rightarrow \infty}\left(\Lambda_{\rho}^{-1 \mu} P^{\rho}-k^{\mu}\right) U^{-1}(\Lambda, a) \Psi_{N}=0 \\
\lim _{N \rightarrow \infty}\left(P^{\rho}-\Lambda_{\mu}{ }^{\rho} k^{\mu}\right) U^{-1}(\Lambda, a) \Psi_{N}=0 .
\end{gathered}
$$

Uma vez que, dado qualquer quadrimomento $k^{\mu}$ satisfazendo $k^{2}=m^{2}$, qualquer outro $k^{\prime \mu}$ também satisfazendo $k^{\prime 2}=m^{2}$ pode ser obtido deste por uma transformação de Lorentz, o espectro de $P^{\mu}$ é o conjunto de todos os $k^{\mu}$ satisfazendo $k^{2}=0$.

A representação mais simples ocorre quando o autoespaço associado a $k^{\mu}$ é unidimensional. $P^{\mu}$ é realizado no espaço de Hilbert das funções de quadrado Lebesgue integrável $\Psi(E, \vec{p})$ definidas no local dos pontos $(E, \vec{p}) \in \mathbb{R}^{4}$ tais que $E^{2}-p^{2}=m^{2}$, chamado de concha de massa. $\hat{H} \Psi(E, \vec{p})=E \Psi(E, \vec{p})$ e $\hat{P}^{i} \Psi(E, \vec{p})=p^{i} \Psi(E, \vec{p})$. O produto interno (definido pela medida de integração 5.59 pode ser escolhido relativisticamente invariante pela escolha:

$$
(\Psi(E, \vec{p}), \Phi(E, \vec{p}))=\int \bar{\Psi}(E, \vec{p}) \Phi(E, \vec{p}) \delta\left(p^{2}+m^{2}\right) \theta\left(p^{0}\right) d^{4} p,
$$

onde as funções acima podem ser estendidas para além da concha de massa produzindo uma expressão que, contudo, não depende desta estensão. Que define a seguinte realização do grupo de Poincaré (nesse momento, não vamos entrar na questão da representação de uma álgebra não comutativa, veja a construção GNS no capítulo 6):

$$
\Psi\left(p^{\mu}\right) \stackrel{\left(\Lambda, x^{\mu}\right)}{\longrightarrow} e^{i p^{\mu} x_{\mu}} \Psi\left(\Lambda_{\nu}^{-1 \mu} p^{\nu}\right)
$$

Alternativamente, podemos representar o espaço de Hilbert usando as funções $\psi(\vec{p})=$ $\Psi(E, \vec{p})$ com produto interno:

$$
(\psi(\vec{p}), \phi(\vec{p}))=\int \bar{\psi}(\vec{p}) \phi(\vec{p}) \frac{d^{3} p}{2 E}
$$

o que já nos mostra a inadequação do conceito de localização no contexto relativístico ao tentar incluir um operador $x^{i}$ tal que $\left[x^{i}, p^{j}\right]=i \delta^{i j}$, o que se torna trivial adotando a função de onda de Newton-Wigner, que na representação de momento se escreve $\psi_{N W}(\vec{p})=\frac{\psi(\vec{p})}{\sqrt{2 E}}$. De fato, um estado quântico localizado em $x^{\prime}$, descrito por uma delta de Dirac na representação de posição (Fourier transformado) $\delta\left(x-x^{\prime}\right)$, corresponde, na 
representação de momento, a $\psi_{N W}(\vec{p}) \propto e^{i p x}$, que se transforma, via $\Lambda^{-1}$, em $e^{i x_{i} \Lambda^{i}{ }_{0} \bar{E}} e^{i x_{i} \bar{p}^{i}}$, não mais uma onda plana, logo, não mais localizado na representação conjugada.

Outra maneira de caracterizar essa representação é considerá-la uma distribuição $\mathcal{S}^{*}\left(\mathbb{R}^{4}\right)^{18}$ com suporte concentrado na concha de massa 19 . Para estas está definida a transformada de Fourier:

$$
\Psi(x) \equiv(2 \pi)^{-\frac{3}{2}} \int \Psi(E, p) e^{-i p^{\mu} x \mu} \delta\left(p^{2}+m^{2}\right) \theta\left(p^{0}\right) d^{4} p,
$$

sendo esta definição equivalente àquela dada pela dualidade:

$$
(2 \pi)^{n}(\tilde{F}, \dot{\tilde{\phi}}) \equiv(F, \phi)
$$

que implica na seguinte definição para a transformada de Fourier:

$$
(\mathcal{F}[F], \phi) \equiv \frac{1}{(2 \pi)^{n}}\left(F, \mathcal{F}^{-1}[\phi]\right)
$$

onde $F \in \mathcal{S}^{*}\left(\mathbb{R}^{4}\right)$ é um funcional linear de $\mathcal{S}\left(\mathbb{R}^{4}\right), \phi(x)=\phi(-x)$, $\tilde{\phi}$ é a transformada da Fourier de $\phi, n$ é a dimensão do espaço $\mathbb{R}^{n}$ em que estão definidas as funções teste. Isso justifica o emprego de $F \in \mathcal{S}^{*}\left(\mathbb{R}^{4}\right)$, pois esta definição implica que a tranformada de Fourier pertence ao mesmo espaço de distribuições, ou seja: $\tilde{F} \in \mathcal{S}^{*}\left(\mathbb{R}^{4}\right)$. O que não é válido para outras classes de funções generalizadas. Escrever a transformada da forma 5.70 torna a sua regra de transformação manifestadamente covariante. Isso nos leva ao chamado Campo escalar, ou função de onda covariante, satisfazendo a equação de Klein-Gordon:

$$
\left(\square+m^{2}\right) \Psi(x)=0
$$

Observe que essa transformada de Fourier é, exatamente como propunha a motivação original para formulação das equações de campo relativísticas, apenas outra representação para os elementos que compõem espaço de Hilbert, uma representação em que a translação temporal é trivialmente realizada: $\Psi(t, \vec{x}) \rightarrow \Psi\left(t+t^{\prime}, \vec{x}\right)$, e não a operação inversa ao esquema de quantização de campo anteriormente considerada no capítulo anterior. De

\footnotetext{
${ }^{18} \mathrm{O}$ estado quântico $\Phi(E, \vec{p})$ é transformado num funcional linear através de $\int \Phi(E, \vec{p}) f(x) \delta\left(p^{2}+\right.$ $\left.m^{2}\right) \theta\left(p^{0}\right) d^{4} p, f \in S\left(\mathbb{R}^{4}\right)$.

${ }^{19} \mathrm{O}$ suporte de uma função é o conjunto fechado fora do qual o função se anula. Para uma distribuição $\phi$, o suporte é o conjunto $V \in \mathbb{R}^{n}$ dos pontos tais que se o suporte $M$ da função teste $f$ é tal que $M \cap V=\emptyset$, então $(\phi, f)=0$
} 
fato, a transformada de Fourier é uma transformação unitária no espaço $L^{2}$. O produto interno nessa representação é:

$$
\langle\Psi \mid \Phi\rangle=i \int_{x^{0}=t}\left(\bar{\Psi} \frac{\partial \Phi}{\partial x^{0}}-\frac{\partial \bar{\Psi}}{\partial x^{0}} \Phi\right) d^{3} x .
$$

Observe ainda que $\Psi(x)$ não é a função de Newton-Wigner, estas são relacionadas por:

$$
\Psi_{N W}=\int K\left(x-x^{\prime}\right) \Psi\left(t, x^{\prime}\right) d^{3} x^{\prime}
$$

onde $K$ é a transformada de Fourier de $(2 E(p))^{-\frac{1}{2}}$, uma função de rápido decrescimento de $m\left|x-x^{\prime}\right|$. A função de onda covariante, por tanto, nos diz aproximadamente onde está a partícula se uma precisão não maior que o comprimento de onda de Compton, $m^{-1}$, é necessária. O conceito de localização não é apropriado para partículas não massivas.

Para construir a representação mais geral, devemos relaxar a condição de que o autoespaço de cada autovalor é unidimensional. Nesse caso, a princípio, um dado autovalor $k^{\mu}$ pode ter um número arbitrário de autoestados generalizados linearmente independentes, sendo o número máximo diferente para cada $k^{\mu}$. Mas essa possibilidade pode ser excluída. De fato, suponha que $k^{\mu}$ tenha $N$ autoestados ortogonais:

$$
\lim _{N}\left(P^{\mu}-k^{\mu}\right) \Psi_{N}^{j} \rightarrow 0, j=1, \cdots, N \operatorname{com} \lim _{N}\left(\Psi_{N}^{i}, \Psi_{N}^{j}\right) \rightarrow 0
$$

Mas $U^{-1}(\Lambda, a) \Psi_{N}^{j}$ é autoestado generalizado de $P^{\mu}$ com autovalor $\Lambda_{\mu}{ }^{\rho} k^{\mu} \mathrm{e}$

$$
\lim _{N}\left(U^{-1}(\Lambda, a) \Psi_{N}^{i}, U^{-1}(\Lambda, a) \Psi_{N}^{j}\right) \rightarrow 0
$$

Desse modo, o conjunto dos autoestados generalizados pode ser escrito como:

$$
\Psi_{k^{\mu} \sigma}
$$

onde sigma é um índice discreto que pode assumir infinitos valores ${ }^{20}$, associados a graus de liberdade internos das partículas, mas em aplicações físicas, como dito, postulamos a inexistência de graus de liberdade internos contínuos, o que equivale a considerar apenas um número finito de possíveis valores para $\sigma$.

\footnotetext{
${ }^{20}$ Observe que não estamos considerando a possibilidade de existir um conjunto não contável de autoestados generalizados linearmente independentes associados a um mesmo autovalor, mas essa possibilidade é excluída pelo requerimento de separabilidade do espaço de Hilbert
} 
Facilitará a análise decompor $U(\Lambda)$ em partes independentes que atuam nos índices $p^{\mu}$ e $\sigma$. Para tanto, observemos que a ação do grupo de Poincaré é transitiva na concha de massa, ou seja, qualquer vetor $k^{\mu}$ desta pode ser transformado em qualquer outro, digamos $p^{\mu}$, pela ação de uma transformação de Lorentz $L\left(p^{\mu}\right)$. Dada uma representação do grupo de Poincaré, definida na base $\Psi_{k^{\prime \mu} \sigma}$, podemos escolher um autoestado generalizado $\Psi_{k^{\mu} \sigma} \mathrm{e}$ definir uma nova base através de

$$
\Psi_{p^{\mu} \sigma}=N\left(p^{\mu}\right) U\left(L\left(p^{\mu}\right)\right) \Psi_{k^{\mu} \sigma}
$$

Podemos definir de várias maneiras a ação dos operadores $U(\Lambda)$ nos estados generalizados, por exemplo:

$$
(U(\Lambda) \Psi, \phi)=\left(\Psi, U(\Lambda)^{-1} \phi\right)
$$

onde $\phi \in \Omega$, ou:

$$
U(\Lambda) \Psi=\left\{U(\Lambda) \Psi_{n}\right\}
$$

isto é, atuar os operadores $U(\Lambda)$ em cada um dos elementos de qualquer sequência $\Psi_{n}$ que define a classe de equivalência.

$N\left(p^{\mu}\right)$ é uma normalização, que afeta a definição do produto interno. O produto interno $\left(\Psi_{p^{\mu} \sigma}, \Psi_{p^{\prime \mu} \sigma^{\prime}}\right)$ é definido pelo teorema kernel de Swartz, que diz que todo funcional bilinear contínuo no espaço de funções teste $\mathcal{S}(\Omega)$ faz corresponder uma distribuição que a define, deste modo:

$$
(\Psi, \Phi)=\sum_{\sigma \sigma^{\prime}} \int d^{3} p d^{3} p^{\prime}\left(\Psi_{p^{\mu} \sigma}, \Psi_{p^{\prime \mu} \sigma^{\prime}}\right) \Psi_{\sigma}\left(p^{\mu}\right) \Phi_{\sigma^{\prime}}\left(p^{\prime \mu}\right)
$$

onde $\Psi$ é um elemento de $\sum_{\sigma}{ }^{\oplus} L_{\sigma}^{2}\left(\mathbb{R}^{3}\right)$ representado pelas funções teste $\psi_{\sigma}(p)$. Logicamente, podemos escolher a medida de integração relativisticamente invariante $\frac{d^{3} p}{2 E(p)} \frac{d^{3} p^{\prime}}{2 E\left(p^{\prime}\right)} \mathrm{e}$ adotarmos uma normalização relativisticamente invariante escolhendo $\left(\Psi_{p^{\mu} \sigma}, \Psi_{p^{\prime \mu} \sigma^{\prime}}\right)$ uma distribuição com tal simetria.

A nova base tem a vantagem de que $U\left(L\left(p^{\mu}\right)\right)$ passa a afetar apenas os índices $p^{\mu}$. Fatoremos, então, uma transformação qualquer em termos de transformações independentes nos índices:

$$
U(\Lambda) \Psi_{p^{\mu} \sigma}=N\left(p^{\mu}\right) U\left(\Lambda L\left(p^{\mu}\right)\right) \Psi_{k^{\mu} \sigma}=N\left(p^{\mu}\right) U(L(\Lambda p)) U\left(L^{-1}(\Lambda p) \Lambda L\left(p^{\mu}\right)\right) \Psi_{k^{\mu} \sigma}
$$


Uma vez que $p^{0} \delta^{3}\left(p^{\prime}-p\right)=k^{0} \delta^{3}\left(k^{\prime}-k\right)$ e $\left(\Psi_{p^{\prime}, \sigma^{\prime}}, \Psi_{p, \sigma}\right)=|N(p)|^{2} \delta_{\sigma \sigma^{\prime}} \delta^{3}\left(k^{\prime}-k\right)$, a normalização $N(p)=\sqrt{\frac{k^{0}}{p^{0}}}$ torna a nova base ortonormal:

$$
\left(\Psi_{p^{\prime}, \sigma^{\prime}}, \Psi_{p, \sigma}\right)=\delta_{\sigma^{\prime} \sigma} \delta^{3}\left(p^{\prime}-p\right)
$$

A transformação de Lorentz

$$
W(\Lambda, p)=L^{-1}(\Lambda p) \Lambda L\left(p^{\mu}\right)
$$

deixa invariante $k^{\mu}$, todas as transformações desse tipo formam juntas um grupo que só pode, por tanto, afetar os índices $\sigma$. Este é chamado de pequeno grupo e toda representação irredutível do grupo de Poincaré implica numa representação irredutível do pequeno grupo. Para a representação que satisfaz $P^{2}=m^{2}>0$, que representa o espaço de Hilbert de uma partícula massiva, podemos escolher $k=(1,0,0,0)$, que imediatamente nos mostra que esse grupo é $S O(3)$, das rotações em três dimensões; para $P^{2}=0$ e $P^{\mu} \neq 0$, as partículas sem massa, podemos escolher $k=(1,0,0,1)$ e usar a correspondência 5.39 entre o grupo de Lorentz próprio e $S L(2, \mathbb{C})$, que faz corresponder a $k$ a matriz $\bar{p}$

$$
\bar{p}=\left(\begin{array}{ll}
1 & 0 \\
0 & 0
\end{array}\right)
$$

que é deixada invariante pelos subgrupos

$$
\gamma_{\phi}=\left(\begin{array}{cc}
e^{i \phi} & 0 \\
0 & e^{-i \phi}
\end{array}\right), \quad \gamma_{\eta}=\left(\begin{array}{cc}
1 & \eta \\
0 & 1
\end{array}\right)
$$

$\gamma_{\phi}, \phi$ real $(\bmod 2 \pi)$, é uma representação do grupo de rotações em duas dimensões, enquanto $\gamma_{\eta}, \eta$ complexo, do grupo de translações. O pequeno grupo associado é, por tanto, $I S O(2)$ de rotações e translações em $\mathbb{R}^{2}$; para $P^{2}=0$ e $P^{\mu}=0$, que representa o vácuo, um estado sem partículas, este é o próprio grupo de Lorentz $S L(2, \mathbb{C})$.

A teoria de grupos, contudo, apenas assegura que todas as representações irredutíveis inequivalentes são de dimensão finita se o grupo de Lie associado for compacto, o que grosseiramente significa que o espaço de parâmetros tem tamanho finito. Para essas, a teoria assegura ainda que existe apenas uma coleção enumerável de representações irredutíveis inequivalentes que formam o sistema representativo. Esse é o caso de $S O(3)$. De fato, podemos parametrizar os seus elementos através de uma rotação por um ângulo $\xi$ em torno 
do vetor que aponta na direção $(\theta, \phi)$, por tanto, a variedade do grupo é uma esfera de raio $\pi$, cujos pontos antipodais na superfície são identificados. Já podemos antecipar daqui que necessitamos de condições adicionais para gerar representações de dimensão finita para $I S O(2)$ e $S L(2, \mathbb{C})$. Em particular, todas as representações (unitárias) do pequeno grupo do vácuo são de dimensão infinita, exceto a trivial que manda todos os elementos do grupo na identidade $\AA^{21}$. Isso nos leva ao seguinte postulado:

Postulado 2 (Condição espectral b). O ponto $p=0$ é um autovalor discreto não degenerado do operador $P$. Ou seja, existe um único estado $\Psi_{0}$ no espaço $\mathcal{H}$ para o qual $P \Psi_{0}=0$. Esse estado é invariante pela ação do grupo de Lorentz, ou seja $U(\{\Lambda, a\}) \Psi_{0}=\Psi_{0}$ para todo $\{\Lambda, a\} \in \mathcal{P}_{0}$

Quanto a $I S O(2)$, a sua parte não compacta, associada à parte de translações, só admite representações de dimensão infinita, associadas às representações contínuas de spin, exceto pela representação trivial. Assumimos então que esse subgrupo é mapeado da representação trivial. Sobra então a representação de um subgrupo de rotação em torno de um eixo. Esse eixo resulta ser a direção do momento linear, visto que $\gamma_{\phi}$ é uma rotação no plano $x_{1}-x_{2}$ por um ângulo $\theta=2 \phi$ e o vetor $k$ aponta na direção $x_{3}$. Esse subgrupo tem como gerador a projeção do spin na direção do momento, chamada de helicidade, que denotamos $J_{3}$, e os possíveis valores para a helicidade são discretos pelo fato de todas as representações de $\gamma_{\phi}$, subgrupo do grupo de cobertura, satisfazerem $U(\phi)=U(\phi+2 \pi)$, ou, equivalentemente, $U(\theta)=U(\theta+4 \pi)$. Desse modo, os possíveis valores para a helicidade, denotada $\sigma$, são, pelo argumento do teorema de Stone, $\sigma=\frac{n}{2}, n$ inteiro. Podemos escolher a representação em que $J_{3}$ é diagonal, todas as demais sendo unitariamente equivalentes $\theta(W(\Lambda, p))$ sendo o ângulo de rotação associado a $W(\Lambda, p)$ :

$$
U(\Lambda) \Psi_{p^{\mu} \sigma}=\left(\frac{(\Lambda p)^{0}}{p^{0}}\right) e^{i \sigma \theta(W(\Lambda, p))} \Psi_{\Lambda p, \sigma}
$$

Observe que se considerarmos apenas a representação de $P_{+}^{\uparrow}$, um único autoestado de $J_{3}$, associado a um dado valor de $\sigma$, já produziria uma representação irredutível, mas se considerarmos as inversões espaciais, estas conectam partículas de helicidades opostas, mas

\footnotetext{
${ }^{21}$ Isso decorre do fato de que o espectro do quadrimomento é ilimitado, implicando num operador descontínuo, mas todo operador linear no espaço de Hilbert de dimensão finita é limitado
} 
nem todas as interações observadas na natureza obedecem tal simetria. O eletromagnetismo, contudo, obedece a simetria de inversão espacial, o que nos leva a considerar estados de helicidades \pm 1 como definindo o espaço de Hilbert dos fótons, enquanto as partículas emitidas no decaimento beta, de helicidades $\pm \frac{1}{2}$, que interagem via forças que não obedecem a essa simetria, são consideradas partículas diferentes (neutrinos e antineutrinos).

Quanto a $S O(3)$, pelo teorema de Stone, todo elemento é da forma $e^{i \theta^{i} L_{i}}$, enquanto que $\left[L_{i}, L_{j}\right]=i \epsilon_{i j k} L_{k}$, uma álgebra de Lie que tem como Casimir $L^{2}=\sum_{i} L_{i}^{2}$. Observe que este Casimir já estava indiretamente presente em 5.53 , pois $W=m^{2} L^{2}$. Podemos então representar $L_{i}$ e resolver completamente o problema de representar $S O(3)$. Representar operadores autoadjuntos, como frisado anteriormente, começa por representar subálgebras de operadores que comutam, operadores convenientes são o próprio Casimir $L^{2}$, que é múltiplo da identidade na representação irredutível, e $L_{3}$. Supondo que dispomos dessa representação, dado um autoestado de $L_{3}, \Psi_{l_{3}}$, tal que $L_{3} \Psi_{l_{3}}=l_{3} \Psi_{l_{3}}$, temos que $L_{3} L_{ \pm} \Psi_{l_{3}}=$ $\left(l_{3} \pm 1\right) L_{ \pm} \Psi_{l_{3}}, L_{ \pm}=L_{1} \pm i L_{2}$. uma vez que $L^{2} \geq L_{3}$, deve existir $l$ tal que $\Psi_{l}$ satisfaz $L_{+} \Psi_{l}=0$ e $k$ tal que $\Psi_{k}$ satisfaz $L_{-} \Psi_{k}=0$. Temos que

$$
\begin{gathered}
\left(\Psi_{l}, L_{-} L_{+} \Psi_{l}\right)=\left(\Psi_{l},\left(L^{2}-L_{3}^{2}-L_{3}\right) \Psi_{l}\right)=\lambda^{2}-l(l+1)=0 \\
\left(\Psi_{k}, L_{+} L_{-} \Psi_{k}\right)=\left(\Psi_{k},\left(L^{2}-L_{3}^{2}+L_{3}\right) \Psi_{k}\right)=\lambda^{2}-k(k-1)=0,
\end{gathered}
$$

que implica $k=-l$, quando impomos $l>k$. O espectro de $L_{3}$ é então $-l,-l+1, \cdots l-1, l$, que nos diz que a representação do pequeno grupo é de dimensão $2 l+1$. Podemos, desse modo, definir a representação do pequeno grupo tal que $e^{\omega_{i j}}=W(\Lambda, p)$ e definir a matriz $D_{\sigma \sigma^{\prime}}^{l}(W(\Lambda, p))=e^{\frac{i}{2} \omega_{i j} M^{i j}}\left[M^{12}=L_{3}, M^{23}=L_{1}\right.$ e $M^{31}=L_{2}$ (observe que já aproveitamos da definição anterior do tensor de momento angular em 5.51. Uma solução analítica é conhecida para esse problema: Coloquemos $W(\Lambda, p)$ da forma $S L(2, C)$, via 5.39 , denotada $V$, tal que

$$
V=\left(\begin{array}{cc}
v_{1} & v_{2} \\
-\overline{v_{2}} & \overline{v_{1}}
\end{array}\right), \quad \operatorname{det} V=\left|v_{1}\right|^{2}+\left|v_{2}\right|^{2}=1
$$

definamos então

$$
D_{\sigma, \sigma^{\prime}}^{(l)}(V)=\sqrt{\frac{(l+\sigma) !(l-\sigma) !}{\left(l+\sigma^{\prime}\right) !\left(l-\sigma^{\prime}\right) !}} v_{1}^{\sigma+\sigma^{\prime}} v_{2}^{\sigma-\sigma^{\prime}} P_{l-\sigma}^{\left(\sigma-\sigma^{\prime}, \sigma+\sigma^{\prime}\right)}\left(v_{1} \overline{v_{1}}-v_{2} \overline{v_{2}}\right),
$$

\footnotetext{
${ }^{22}$ Observe que usamos a notação empregada na definição do tensor de momento angular, de fato, uma das subálgebras de Poincaré é $S O(3)$.
} 
em que $P_{n}^{(a, b)}(x)$ são os polinômios de Jacobi:

$$
P_{n}^{(a, b)}(x)=\frac{(-1)^{n}}{2^{n} n !}(1-x)^{-a}(1+x)^{-b} \frac{d^{n}}{d x^{n}}\left[(1-x)^{a+n}(1+x)^{b+n}\right] .
$$

Sendo assim:

$$
U(\Lambda) \Psi_{p^{\mu} \sigma}=\left(\frac{(\Lambda p)^{0}}{p^{0}}\right) \sum_{\sigma^{\prime}} D_{\sigma \sigma^{\prime}}^{(l)}(V(\Lambda, p)) \Psi_{\Lambda p, \sigma^{\prime}}
$$

Observe que se tentarmos aplicar a transformada de Fourier na função de estado $\psi_{\sigma}(p)$, que descreve o estado na base $\Psi_{p^{\mu} \sigma}$, cuja realização de Lorentz é dada por 5.88 e 5.94 . teremos uma regra de transformação muito complexa no espaço de posição devido a dependência não trivial das matrizes $D_{\sigma \sigma^{\prime}}$ com o momento. Podemos, no caso de spin $1 / 2$, adotar uma descrição alternativa dos estados (adotando uma normalização $N(p)=1$ em 5.79 :

$$
\tilde{\phi}_{\tau}(p)=m^{\frac{1}{2}}(E(p)-p \sigma)_{\tau \sigma}^{-\frac{1}{2}} \psi_{\sigma}(p)
$$

A transformada de Fourier desta segue a regra de transformação spinorial $5.41{ }^{23}$.

$$
(U(\alpha) \phi)_{\tau}(x)=\alpha_{\tau \sigma} \phi_{\sigma}\left(\Lambda^{-1}(\alpha) x\right)
$$

$\alpha \in S L(2, \mathbb{C})$. Podemos introduzir também o spinor conjugado contravariante.

$$
(E(p)-p \sigma) \tilde{\phi}_{\tau}(p)=m \tilde{\chi}
$$

cuja regra de transformação é deduzida de maneira análoga ao footnote 23 , mas ao invés de partir da representação spinorial para construir uma representação de $W(\Lambda, p)$, partimos da representação contragradiente conjugada. A representação irredutível obtida deve ser equivalente. Podemos então construir a função de onda de quatro componentes:

$$
\tilde{\psi}=\left(\begin{array}{c}
\tilde{\phi} \\
\tilde{\chi}
\end{array}\right)
$$

que no espaço de posição satisfaz a equação de Dirac:

$$
\left(i \gamma^{\mu} \partial_{\mu}+m\right) \psi=0
$$

\footnotetext{
${ }^{23}$ Observe que a representação spinorial de $S L(2, \mathbb{C}), 5.41$, não é unitária, mas se nos restringirmos ao subgrupo de rotações, $S O(3)$, está é e fornece uma representação unitária irredutível. Para esta, $L(p)=(E+\sigma p)^{\frac{1}{2}}$, de modo que $W(\Lambda, p)=L(\Lambda p)^{-1} \Lambda L(p)=\left(E^{\prime}+\sigma p^{\prime}\right)^{-\frac{1}{2}} \alpha(E+\sigma p)^{\frac{1}{2}}$, onde $p^{\prime}=\Lambda p$. Por tanto, $\left(E^{\prime}+\sigma p^{\prime}\right)^{\frac{1}{2}} \tilde{\Psi}\left(p^{\prime}\right)=\alpha(E+\sigma p)^{\frac{1}{2}} \tilde{\Psi}(p)$. Observe que $(E+\sigma p)^{\frac{1}{2}}=(E-\sigma p)^{-\frac{1}{2}}$.
} 


$$
\gamma^{0}=\left(\begin{array}{cc}
0 & 1 \\
1 & 0
\end{array}\right) ; \quad \gamma^{i}=\left(\begin{array}{cc}
0 & -\sigma_{i} \\
\sigma_{i} & 0
\end{array}\right)
$$

que se transforma segundo:

$$
(U(\alpha) \psi)(x)=S(\alpha) \psi\left(\Lambda^{-1}(\alpha) x\right)
$$

onde:

$$
S(\alpha)=\left(\begin{array}{cc}
\alpha & 0 \\
0 & \alpha^{*-1}
\end{array}\right)
$$

sendo o produto interno dessa representação:

$$
\langle\psi \mid \psi\rangle=\int_{x^{0}=t} \psi^{*} \psi d^{3} x
$$

Observe que, ao iniciar o tratamento do problema da representação de grupos, enumeramos as questões fundamentais envolvidas no problema:

1. Como diferenciar tais representações?

2. Quais as mais simples?

3. Quantas representações diferentes existem?

4. Qual a mais geral possível?

Destas, apenas a última não foi respondida pela argumentação que se seguiu. Abordaremos esta questão no capítulo posterior e veremos que ela é um dos pontos centrais no raciocínio que levou a um dos resultados originais desta tese.

Outro ponto fundamental é que afirmamos que a prescrição de Wigner determina completamente a teoria da matéria, em outras palavras, o conhecimento da representação do grupo de Poincaré que descreve a matéria é suficiente para descrevê-la (na ausência de gravidade). Por que a introdução do conceito de campo? Como discutido no capítulo 2 , o campo é mecanismo através do qual o requerimento de localidade é implementado na física, a teoria quântica de campos é o resultado da quantização de uma física local. Existe mais informação nos campos quânticos que uma representação do grupo de Poincaré? O campo é na verdade um passo intermediário para construir os estados do espaço de Hilbert, onde atua a representação de Poincaré, a partir de um único estado, o vácuo, que 
é assumido fazer parte de cada representação de Poincaré. Em outras palavras, todo o espaço de Hilbert é gerado pela expansão linear de expressões do tipo:

$$
\int d^{4} x_{1} d^{4} x_{2} \cdots d^{4} x_{N} f_{i}\left(x_{1}\right) f_{j}\left(x_{2}\right) \cdots f_{k}\left(x_{N-1}\right) f_{l}\left(x_{N}\right) \Phi_{i}^{*}\left(x_{1}\right) \Phi_{j}^{*}\left(x_{2}\right) \cdots \Phi_{k}\left(x_{N-1}\right) \Phi_{l}\left(x_{N}\right)|0\rangle
$$

onde as integrais são entendidas no sentido distribucional, ou seja $\langle\psi|\Phi(x)| \phi\rangle$ é uma distribuição que atua como funcional linear nas funções teste $f_{i}$ e não uma função ordinária. Nesse sentido, dizemos que o vácuo $|0\rangle$ é cíclico com respeito à ação dos operadores de campo.

Se o campo é uma observável, ele é utilizado para exprimir um requerimento adicional na representação do grupo de Poincaré que ocorre em física, a localidade relativística:

$$
\left[\phi_{i}(x), \phi_{j}(y)\right]=0, \text { se }(x-y)^{2} \leq 0
$$

Dado o conhecimento de $U(A, a)$, o campo é completamente reconstruído pelo requerimento de covariância relativística 24

$$
\phi_{i}\left(V_{j i}(A) f_{i}(\Lambda x+a)\right)=U^{-1}(A, a) \phi_{j}(x) U(A, a),
$$

onde $V_{j i}$ é uma representação de dimensão finita de $A \in S L(2, \mathbb{C})$ e $\int d^{4} x \phi_{i}(x) f_{i}(x) \equiv$ $\phi_{i}\left(f_{i}\right)$. Nem todos os campos são observáveis, apenas aqueles que se transformam por representações tensoriais de $S L(2, \mathbb{C})$, para as quais:

$$
U(-1, a)=\epsilon U(1, a), \quad \epsilon=1
$$

sendo -1 a matriz de $S L(2, \mathbb{C})$ correspondente a uma rotação de $2 \pi$ em torno do eixo $z$. De um modo geral, $\epsilon= \pm 1$, mas o valor de $\epsilon$ define subespaços coerentes, associados às regras de superseleção. Como argumentado, observáveis deixam invariantes subespaços coerentes, mas campos tais que $\epsilon=-1$ mapeiam subespaços com valores de $\epsilon$ diferentes. Isso ilustra o fato de que representações projetivas levam a regras de superseleção na teoria. Para estes:

$$
\left\{\Psi_{\alpha}(x), \Psi_{\beta}(y)\right\}=0, \text { se }(x-y)^{2} \leq 0
$$

\footnotetext{
${ }^{24}$ Como argumentado na subseção $4.4 .2, \phi(x)$ não é um operador no espaço de Hilbert, apenas $\phi(f(x))$, para a função teste $f(x)$. É possível mostrar que a equação $\phi(\Lambda x+a)=U^{-1}(A, a) \phi(x) U(A, a)$, sendo $\phi(x)$ um operador autoadjunto num domínio $\mathcal{D}$ do espaço de Hilbert que contém o vácuo como vetor cíclico, só admite uma realização: No espaço de Hilbert de dimensão 1 e satisfazendo $\phi(x)|0\rangle=$ const $|0\rangle$
} 
O que implica que formas quadráticas em $\Psi_{\alpha}$, as quais se transformam tensorialmente, sendo observáveis, satisfazem 5.105 . 


\section{Capítulo 6}

\section{Espaços não comutativos, teoria quântica e a simetria relativística}

\subsection{Introdução}

Nesse capítulo, discutimos a relevância da álgebra para a física. Mostramos que admitir uma estrutura matemática relativamente geral para uma teoria física, onde os dois ingredientes básicos são observáveis e estados, é suficiente para lançar uma descrição algébrica das mesmas. Esta descrição algébrica é suficiente para codificar a teoria, decorre dela que existem essencialmente dois tipos de teorias físicas: as clássicas e as quânticas, ainda que nunca tivéssemos ouvido falar de princípio da incerteza. A álgebra também se mostra capaz de codificar a noção de um contínuo de pontos, o espaço topológico, estrutura matemática que descreve o espaço-tempo conforme discutido no cap. 2, bem como boa parte da matemática que se pode fazer nele. Desta descrição algébrica, decorre uma dicotomia similar àquela das teorias físicas: espaço clássico e espaço quântico, ou, equivalentemente, espaço comutativo e não comutativo. Exploramos aqui a formulação da física sob hipótese de espaço-tempo não comutativo para tratar a questão de se a física deste espaço é relativística ou não e o que, nesse contexto, se poderia entender por "ser relativística".

A motivação para esta análise é que o modelo fundamental tratado nessa tese, a inflação não comutativa Alexander et al. (2003), lançou-se na literatura como uma possível até então elusiva conexão entre teoria de cordas e inflação através de uma previsão da teoria de cordas de que as teorias não comutativas são a primeira modificação efetiva da teoria de campos usual em altas energias. Não apenas, a ideia de espaço não comutativo é argumentada por alguns autores como uma característica esperada de uma teoria quântica da gravidade 
Szabo (2010). A ideia da inflação não comutativa é que o caráter não relativístico dessas teorias seria o gatilho da inflação. Apesar disso, sua formulação original era puramente fenomenológica e heurística, carecendo de justificativas rigorosas para a questão de se suas premissas são compatíveis ou não com o modelo que se seguiu e se existe de fato algum espaço-tempo não comutativo que o implementa. A inflação não comutativa é, contudo, um modelo com vantagens conceituais em relação à implementação discutida nos capítulos 3 e 4. notadamente, consegue fazer com que a inflação seja consequência natural das leis físicas derradeiras, tal como se desejava ao tentar mergulhar a inflação nos modelos de grande unificação, conforme o discutido na introdução. É desejável tornar esse modelo mais robusto e autoconsistente e que a inflação não comutativa seja realizável a partir de um cenário não comutativo. As principais referências para este capítulo são: Strocchi (2008), Haag (1996), Bogolubov et al. (1975), de Faria e de Melo (2010), Amelino-Camelia e Arzano (2002), Landsman (1998), Banerjee et al. (2004), Sattinger e Weaver (2010), Douglas e Nekrasov (2001), Carmona et al. (2003), Szabo (2010), Gamboa et al. (2001)

\subsection{A abordagem das álgebras $C^{*}$ para a teoria quântica}

A ideia por trás do conceito de grupo, discutido no capítulo anterior, é que existem ao menos duas maneiras de definir uma coleção $\mathfrak{g}$ de transformações que atuam em algum espaço $X$, escolhidas dentre um conjunto maior de transformações inversíveis que denotamos $L(X)$. A primeira é especificar cada uma das transformações explicitamente, a segunda é considerar essas transformações um conjunto $\mathbb{G}$ de incógnitas e definir a chamada operação de multiplicação de grupo, que leva dois elementos dados a um terceiro segundo regras que seguem as propriedades da composição de transformações em $L(X)$, quando então nos perguntamos o quanto esta estrutura restringe a regra $\pi: \mathbb{G} \rightarrow L(X)$ que a respeita, chamada de homomorfismo. Dependendo de tal estrutura, $\mathfrak{g}$ pode estar completamente especificado. Um procedimento essencialmente equivalente a definir um conjunto de equações (algébricas) a determinar um conjunto de incógnitas. Este é o ponto de vista da álgebra, concentrar-se nas operações definidas entre os elementos como potenciais provedoras de informação para especificá-los, parte da praxe matemática de expressar a mesma informação de um modo alternativo, explorando conexões a princípio não óbvias entre diferentes conceitos. Na nova descrição, ocasionalmente, problemas antes intratáveis 
demonstram-se factíveis.

Nos capítulos anteriores, abordamos muitos aspectos da teoria quântica. Toda a discussão se fazia em torno da ideia de que existia um espaço de Hilbert onde estavam definidos operadores cuja especificação era parte do problema quântico. O conceito de $C^{*}$-álgebra surge como uma maneira alternativa de falar de teoria quântica sem fazer referência a espaços de Hilbert e seus operadores lineares. Para ilustrar a vantagem lógica deste conceito, observemos a questão da quantização. A princípio, podemos pensar que o salto lógico entre a teoria clássica descrita por funções de variáveis canônicas, por exemplo, e a teoria quântica, descrita por operadores no espaço de Hilbert, parece demasiado contraintuitiva, mas, quando visto do ponto de vista das $C^{*}$-álgebras, a teoria quântica se demonstra uma extensão natural da ideia de teoria clássica. Sendo essas duas descrições dois casos particulares da mais geral estrutura matemática possível para uma teoria física apoiada em requerimentos físicos razoáveis. Mas não apenas, do mesmo modo que o conceito de $C^{*}$-álgebra captura o de espaço de Hilbert, é capaz de fazer o mesmo pelo conceito de espaço topológico, o aparato matemático que captura nossas noções intuitivas de espaço físico. Com extensões, captura ainda o conceito de variedade Riemaniana, descrição do espaço-tempo adotada na relatividade. A $C^{*}$-álgebra é, portanto, o conceito a conectar o contínuo do espaço-tempo (e muito do que se pode fazer nele) tal como o cálculo diferencial e integral e a geometria) à teoria quântica, permitindo um tipo de generalização conceitual semelhante àquele associado à quantização de um sistema clássico, o que conduz ao chamado espaço não comutativo.

Comecemos por demonstrar o poder da álgebra ao codificar a descrição clássica de um sistema físico, definindo um conjunto de operações entre elementos não especificados a partir da qual é possível recuperar o formalismo original. Com efeito, a estrutura matemática de uma teoria física genérica é composta por um conjunto de estados, que denotaremos $\Omega$, e um conjunto de observáveis, que denotaremos $\mathcal{O}$. Um estado deve ser completamente definido pelo conjunto de todas as observações feitas nele. Se, fisicamente, uma observável é definida pelo seu aparato experimental, matematicamente, abstraímos essa situação observando que tal aparato tem como único propósito produzir um número. Podemos, desse modo, considerar uma observável $A \in \mathcal{O}$ como uma regra que leva cada estado $\omega \in \Omega$ num dado valor numérico, ou, alternativamente, considerar um estado como 
um regra que leva cada observável num valor numérico $\omega: \mathcal{O} \rightarrow \mathbb{R}$. Sendo descrições equivalentes, escolheremos esta última. Consideremos o formalismo Hamiltoniano, por exemplo. O estado de um sistema é especificado pelo valor de um conjunto de variáveis canônicas $\Gamma=\left(q_{1}, \cdots, q_{n} ; p_{1}, \cdots, p_{n}\right)$. Por uma observável, entendemos qualquer função contínua $f\left(q_{n} ; p_{n}\right)$. No formalismo que estamos construindo, definamos o estado como o funcional que faz $\omega_{\Gamma}(f)=f\left(q_{n} ; p_{n}\right)$. O que implica ainda que $\omega(1)=1$. Podemos estender o conjunto de funções/observáveis considerado permitindo que assumam valor complexo, o que nos permite definir a operação de conjugação complexa $*: f^{*}=\bar{f}$, que chamaremos de involução, caracterizada pela propriedade $\omega_{\Gamma}\left(f^{*}\right)=\overline{\omega_{\Gamma}(f)}$. Desse modo, estão definidas em $\mathcal{O}$ as operações de multiplicação e combinações lineares a coeficientes complexos por aquelas definidas ponto a ponto. $\mathcal{O}$ é, por tanto, uma álgebra, um conjunto onde estão definidas operações de soma e multiplicação, entre si e por números reais ou complexos.

Podemos definir uma topologia no espaço de observáveis, que define a noção de que certas observáveis são "próximas" de outras, no sentido de que produzem valores próximos para todos os estados. Fazemos isso atrávés de uma norma $\|A\|=\sup _{\Gamma}\left|f\left(q_{n} ; p_{n}\right)\right|$, isto é, duas observáveis $A_{1}$ e $A_{2}$ são arbitrariamente próximas se $\left\|A_{1}-A_{2}\right\|<\epsilon$, com $\epsilon$ arbitrariamente pequeno. É desejável que o conjunto de observáveis seja completo com respeito à noção de convergência considerada. De fato, ser um espaço vetorial completo com respeito à noção de convergência da norma, o que define o chamado espaço de Banach, significa, fisicamente, que podemos sempre construir uma sequência de experimentos que produzem valores tão próximos quanto desejarmos de qualquer outro experimento em qualquer estado, e se definirmos uma sequência $A_{n}$ de observáveis que produzem valores numéricos convergentes para qualquer estado, estamos, na verdade, medindo alguma outra observável.

O conjunto de observáveis $\mathcal{O}$ considerado é completo com respeito à noção de convergência definida pela norma se o conjunto dos $\Gamma=\left(q_{n} ; p_{n}\right)$, o espaço de fase, denotado $X$, é compacto (segundo definição do footnote 14 do capítulo anterior). O produto também é uma operação contínua nessa topologia. A continuidade pode ser mostrada equivalente ao requerimento: $\|A \cdot B\| \leq\|A\| \cdot\|B\|$, tal como em operações lineares em geral. Os estados podem ser identificados com os funcionais contínuos, positivo-definidos e normalizados de $\mathcal{O}$, isto é, contínuo no sentido $|\omega(A)| \leq\|A\|$, positivo-definido significando $\omega\left(A^{*} A\right) \geq 0$ 
e normalizados pelo requerimento $\omega(1)=1$ (visto em retrospectiva, a condição de positividade já assegura as demais). Considerar o conjunto de todos os funcionais contínuos nessa topologia nos leva a considerar um conjunto maior do que o dos pontos $\Gamma$. A forma mais geral dos estados pode, então, ser provadat;

$$
\omega(f)=\int_{X} f d \mu, \quad \omega(1)=\int_{X} 1 d \mu=1
$$

onde $d \mu$ é uma medida de integração positiva definida. Como $d \mu=\rho(x) d^{n} q d^{n} p$, tal que $\rho(x) \geq 0$ é uma função integrável e $\int \rho(x) d^{n} q d^{n} p=1$, é uma possível medida de integração, esta extensão do conjunto de observáveis nos leva a considerar as distribuições de probabilidade no espaço de fases, fundamentais na descrição estatística da mecânica, mas não apenas, é também a descrição de que um dado processo experimental produz um estado sujeito a incertezas experimentais, ou seja, dois estados produzidos pelo mesmo processo podem ter medidas de um dado observável ligeiramente diferentes, apesar de, a princípio, essa imprecisão poder ser tornada arbitrariamente pequena. A um estado de incerteza nula chamemos de estado puro. Que podemos produzir estados experimentalmente apenas sugere que podemos de algum modo influenciar a dinâmica do mundo que observamos. Mais geralmente, as medidas de integração podem ser mostradas em correspondência biunívoca com as distribuições positivo-definidas, isto é, dada uma distribuição $\phi$ tal que $(\phi, f) \geq 0$ para toda função teste $f \geq 0, \int d \mu f=(\phi, f)$ e vice versa. Nesse caso, observe que toda distribuição positiva definida é obtida de um limite da forma $(\phi, f)=\lim _{n \rightarrow \infty} \int d^{n} q d^{n} p \rho_{n}(x) f(x), \rho_{n}(x) \geq 0$, uma função infinitamente diferenciável de suporte compacto, sendo o limite convergente para toda função teste $f$. A medida de integração que é igual a 1 em todo conjunto mensurável que contém o ponto $\Gamma$ e zero em todo que não contém, que equivale à delta de Dirac $\delta(x-\Gamma)$, reproduz os estados $\omega_{\Gamma}$. Observe que $\|A\|=\sup _{\omega}|\omega(A)|$. De modo que a noção de proximidade, ou topologia, previamente definida é equivalente a dizer que duas observáveis $A$ e $A_{n}$ são arbitrariamente próximas quando o estado a produzir o maior valor numérico em módulo na diferença $A-A_{n}$ é arbitrariamente pequeno, ou seja: $\sup _{\omega}\left|\omega\left(A_{n}-A\right)\right|<\epsilon$. Observe ainda que $\left\|A^{*} A\right\|=\|A\|^{2}$.

E quanto à dinâmica? A evolução do sistema clássico é totalmente definida pela Ha-

\footnotetext{
${ }^{1}$ Teorema de Riesz-Markov Strocchi (2008)
} 
miltonia $H\left(q_{n} ; p_{n}\right)$ e pelas equações de Hamilton:

$$
\dot{q}_{n}=\frac{\partial H}{\partial p_{n}} \text { e } \dot{p_{n}}=-\frac{\partial H}{\partial q_{n}} .
$$

Sob condições de regularidade e extensão da dinâmica para todo $t$, estas permitem definir uma transformação de um parâmetro no espaço de observáveis $\mathcal{O}$ nele mesmo:

$$
\alpha_{t} f\left(q_{n} ; p_{n}\right)=f\left(q_{n}(t) ; p_{n}(t)\right)
$$

Essa transformação define o que se chama de *-automorfismo de um parâmetro $t$, que é uma transformação bijetiva $\alpha_{t}$ que satisfaz $\alpha_{t} \circ \alpha_{t}^{\prime}=\alpha_{t+t^{\prime}}$ (regra de composição), preserva toda a estrutura algébrica considerada, ou seja: é linear; preserva o produto: $\alpha_{t}(A B)=$ $\alpha_{t}(A) \alpha_{t}(B)$; preserva normalização: $\alpha_{t}(1)=1$; e preserva involução $\alpha_{t}\left(A^{*}\right)=\alpha_{t}(A)^{*}$. Sob essas condições, pode-se mostrar que ela ainda preserva a norma. Observe ainda que, sob continuidade da evolução Hamiltoniana 6.3, $\omega\left(\alpha_{t} A\right)$ é contínuo para qualquer estado. A essa propriedade chamamos de continuidade fraca.

Suponhamos agora que não sejam especificadas as funções $f\left(q_{n} ; p_{n}\right)$. Temos então apenas um conjunto de símbolos que podem ser somados, multiplicados por números complexos e multiplicados entre si por um produto associativo, i.e. $(a \cdot b) \cdot c=a \cdot(b \cdot c)$, e comutativo, i.e., $a \cdot b=b \cdot a$. Temos ainda a operação de involução, que é antilinear $(A+B)^{*}=A^{*}+B^{*},(\lambda A)^{*}=\bar{\lambda} A^{*}$ e tal que $(A B)^{*}=B^{*} A^{*}$ e $\left(A^{*}\right)^{*}=A$. Temos uma norma que associa um valor $\|A\|$ satisfazendo as propriedades que definem uma norma: $\|A\| \geq 0$ e $\|A\|=0 \Leftrightarrow A=0 ;\|\lambda A\|=|\lambda| \cdot\|A\|$, para $\lambda$ complexo; $\|A+B\| \leq\|A\|+\|B\|$. E essa norma é tal que $\mathcal{O}$ é completo com respeito a eld $2^{2}$ o produto é contínuo e ainda satisfaz $\left\|A^{*} A\right\|=\|A\|^{2}$. O que acabamos de definir foi uma $C^{*}$-álgebra comutativa.

Juntamente com a $C^{*}$-álgebra comutativa, descrição algébrica das observáveis, temos os estados, como funcionais lineares contínuos, positivo-definidos e normalizados. Na verdade, é possível mostrar que, numa $C^{*}$-álgebra com identidade, $\omega\left(A^{*} A\right) \geq 0$ (positividade) se e somente se $\omega$ é limitado e $\|\omega\|=\omega(1)$. Desse modo, basta apenas assumir a positividade, podemos sempre normalizar o estado dividindo-o por sua norma. Nessa formulação, distinguimos dois tipos de estado: o puro, que não pode ser escrito da forma $\omega=\lambda \omega_{1}+(1-\lambda) \omega_{2}$

\footnotetext{
2 i.e. dada uma sequência $a_{n}$ de elementos, se $\lim _{n \rightarrow \infty}\left\|a_{n+m}-a_{n}\right\|=0$ para cada $m>0$, então existe um $a$ tal que $\lim _{n \rightarrow \infty}\left\|a_{n}-a\right\|=0$.
} 
para $\omega_{1}, \omega_{2}$ estados normalizados (de certo modo, linearmente independente de qualquer outro estado). E os estados fiéis, tais que $\omega\left(A^{*} A\right)=0 \Leftrightarrow A=0$.

Decorre que o espaço $X$ e o conjunto de funções complexas contínuas $f\left(q_{n} ; p_{n}\right)$, bem como o conjunto de todas as evoluções Hamiltonianas, podem ser recuperadas a partir da informação especificada acima. Ou seja, operações algébricas definidas entre os elementos codificaram completamente a formulação Hamiltoniana. Este é o chamado teorema de Gelfand-Naimark. O conjunto de todas das possíveis dinâmicas se traduz no conjunto de todos os *-automorfimos contínuos de um parâmetro. Em certo sentido, a $C^{*}$-álgebra comutativa define um sistema de equações que pode ser resolvido de modo a determinar o conjunto de incógnitas $f\left(q_{n}, p_{n}\right)$ unicamente e para o qual é assegurado que exista uma solução.

De fato, se observarmos bem, os funcionais lineares associados aos pontos $\Gamma$ possuem um peculiaridade: são multiplicativos, isto é, $\omega_{\Gamma}(A B)=\omega_{\Gamma}(A) \omega_{\Gamma}(B)$. A ideia do teorema Gelfand-Naimark é mostrar que sempre existem funcionais lineares multiplicativos, cujo conjunto denotamos $\Sigma(\mathcal{A})$, em qualquer $C^{*}$-algebra comutativa $\mathcal{A}$ e que estes reconstroem o conjunto dos pontos e a topologia de $X$, bem como permitem representar os elementos da $C^{*}$-álgebra como funções contínuas no conjunto $\Sigma(\mathcal{A})$, com o valor $\omega(A)$ no ponto $\omega \in \Sigma(\mathcal{A})$. A essa função chamamos de transformada de Gelfand, $\tilde{A}$. A topologia do espaço é reconstruída especificando-se as vizinhanças de cada ponto. Dado um conjunto $A_{i}, i=1, . ., n$ definimos uma vizinhança $U_{A_{i}}(m, \epsilon)=\left\{\bar{m} \in \Sigma(A):\left|\bar{m}\left(A_{i}\right)-m\left(A_{i}\right)\right|<\right.$ $\epsilon, \quad i=1, \cdots, n\}$ de $m \in \Sigma(\mathcal{A})$, ou seja, dois pontos em $\Sigma(\mathcal{A})$ são próximos se produzem valores próximos ao atuarem em qualquer elemento $A \in \mathcal{A}$. Faz parte da demonstração do teorema de Gelfand-Naimark mostrar que, com essa topologia, $\Sigma(\mathcal{A})$ é um espaço topológico compacto e as transformadas de Gelfand mapeiam a $C^{*}$-álgebra no espaço de funções contínuas em $\Sigma(\mathcal{A}), C(\Sigma(\mathcal{A}))$, de maneira a preservar a norma e toda a estrutura algébrica, ou seja, de modo isométrico e isomórfico. Um ponto fundamental do esquema é que o conjunto dos funcionais multiplicativos é inteiramente reconstruído a partir da regra de multiplicação da álgebra. É notável que, se o espaço $X$ tiver buracos, ou outras propriedades intrinsecamente topológicas, as funções definidas em $X$ conterão essa informação.

Feita a identificação dos pontos do espaço com os funcionais multiplicativos 6.1 se 
escreve como:

$$
\omega(A)=\int_{\Sigma(\mathcal{A})} \omega(A) d \mu
$$

que, comparando-se com 5.58, permite uma identificação entre os autoestados generalizados e os funcionais multiplicativos.

Com respeito à dinâmica, o teorema de Liouville nos diz que, sob a evolução Hamiltoniana, a medida de integração $d p_{1} \cdots d p_{n} d q_{1} \cdots d q_{n}$ é invariante. Por outro lado, as medidas de integração definem os estados via 6.1. Para cada $*$-automorfismo contínuo $\alpha_{t}(t)$, podemos definir uma transformação linear $\alpha_{t}^{*}$ que evolui os estados: $\omega\left(\alpha_{t} A\right)=\left(\alpha_{t}^{*} \omega\right)(A)$. Observe que essa definição leva funcionais multiplicativos em funcionais multiplicativos, logo, $\alpha_{t}^{*}$ leva pontos em pontos. Supondo $\omega$ multiplicativo, $\left(\alpha_{t}^{*} \omega\right)(A)$ é contínuo se e somente se $\left(\alpha_{t}^{*} \omega\right)$ for uma curva contínua em $X=\Sigma(\mathcal{A})$. Além disso, $\omega\left(\alpha_{t} A\right)=\left(\alpha_{t}^{*} \omega\right)(A)$ significa que $\left(\alpha_{t} A\right)(\Gamma)=(A)\left(\alpha_{t}^{*} \Gamma\right)$, sendo $\Gamma$ o ponto de $X$ correspondente ao funcional $\omega$, o que implica que $\alpha_{t} A$ é da forma 6.3. Observe que acabamos de definir uma transformação contínua dependente do parâmetro $t$ que leva cada ponto de $X$ ao longo de uma única curva (curvas que não se cruzam) e que leva os conjuntos mensuráveis $\mathcal{A}$ em novos conjuntos mensuráveis $\mathcal{A}^{\prime}$ e tal que $\int_{\mathcal{A}} 1 d \mu=\int_{\mathcal{A}^{\prime}} 1 d \mu$, tal como prevê o teorema de Liouville.

Antes de introduzirmos uma generalização do formalismo anterior, ainda inspirada por formalismos considerados em física, enunciemos uma importante conclusão: Sob certas hipóteses, qualquer conjunto de observáveis físicas $\mathcal{O}$ pode ser representado por um conjunto de operadores autoadjuntos no espaço de Hilbert.

$\mathrm{Na}$ descrição acima, postulamos que uma teoria física tem uma estrutura matemática constituída de estados e observáveis. Decidimos ir além multiplicando e somando observáveis entre si e por números complexos. Porque dispúnhamos de um formalismo Hamiltoniano, essas operações podiam ser definidas de um modo natural. Se não dispuséssemos de tal formalismo, esta estrutura algébrica ainda teria alguma motivação física? De fato, uma observável $A$ leva o estado $\omega$ no número real $\lambda$, que podemos denotar $A(\omega)=\omega(A)=\lambda$. Uma regra que, em última análise, é definida pelo observador que interpreta o instrumento de medida. Podemos então definir a multiplicação por números complexos, produtos e combinações lineares de potências de $A$ como o operador que leva o estado $\omega$ na mesma função de $\lambda$, por exemplo, se $A(\omega)=\lambda, A^{2}(\omega)=\lambda^{2}$, mas não para quaisquer estados, apenas para estados puros, isto é, aqueles que são produzidos por algum 
procedimento experimental de tal modo que a medida de $A$ é sempre a mesma. Aqui, todas as operações são realizadas com números adimensionais. Podemos definir uma involução que nos diz apenas que as observáveis produzem valores reais: $\omega(A)=\overline{\omega(A)}=\omega\left(A^{*}\right)$, que implica $A=A^{*}$. A noção de norma, assim como no caso Hamiltoniano, poderia ser definida como $\sup _{\omega}|\omega(A)|$, que já coloca automaticamente as observáveis satisfazendo $|\omega(A)| \leq\|A\|, \omega\left(A^{*} A\right) \geq 0$ e $\omega(1)=1$, onde 1 é a observável que produz o mesmo valor 1 em qualquer estado. Aqui, está implícita a hipótese

$$
\sup _{\omega}|\omega(A)|<\infty \text {. }
$$

Significando que a medição de uma observável em qualquer estado é limitada. Essa limitação está associada ao fato de que observáveis correspondem a construções experimentais e tais construções têm limites intrínsecos, o resultado de uma medição pode no máximo produzir um valor finito. Vejamos mais adiante, como partindo de construções com remover essa limitação.

Definidos desse modo uma $C^{*}$-álgebra comutativa, $\mathcal{A}_{A}$, gerada por uma única observável. Vejamos agora um maneira alternativa de representar essa $C^{*}$-álgebra.

Pelo teorema Gelfand-Naimark, $A$ é um elemento de uma álgebra de funções contínuas definidas em $\Sigma(\mathcal{A})$. Que conjunto seria esse? Para dar essa resposta, definamos um conceito muito familiar: o espectro $\sigma(A)$ do elemento $A$ na $C^{*}$-álgebra $\mathcal{A}$. Definido como o conjunto dos números complexos $\lambda$ tais que $(A-\lambda 1)$ não possui uma inversa. Observe que essa definição é equivalente a definição de espectro dos operadores lineares de um espaço de Hilbert e enfatiza o papel da multiplicação na sua definição. É possível mostrar que esse conjunto é não vazio para todo elemento $A \in \mathcal{A}$ e, além disso:

$$
\sup _{\lambda \in \sigma(A)}|\lambda|=\|A\|
$$

Ocorre ainda que, dado $A \in \mathcal{A}, \lambda \in \sigma(A)$ se e somente se existir um funcional multiplicativo linear $m \in \Sigma(\mathcal{A})$ tal que:

$$
m(A)=\lambda .
$$

Por tanto, $A$ pode ser entendida como uma função definida no seu espectro, pois se existissem dois funcionais multiplicativos, $m_{1}$ e $m_{2}$ tais que $m_{1}(A)=m_{2}(A)=\lambda \in \sigma(A)$, eles seriam iguais em toda $C^{*}$-álgebra. $A$ é, por tanto, representada como a função que leva 
o ponto $\lambda \in \sigma(A)$ no valor $\lambda$. E quanto aos estados? São definidos por uma medida de integração positivo-definida e normalizada no espectro. Desse modo, $\omega(A)=\int_{\sigma(A)} \lambda d \mu_{\omega}$. Podemos escolher um estado fiel, ou seja, tal que $\omega_{F}\left(A^{*} A\right)=0 \Leftrightarrow A=0$, que corresponde à medida $d \mu_{F}$. Mostraremos mais adiante que este estado sempre existe. Observe que podemos escrever outros estados $\omega$ da forma $\omega(A)=\int_{\sigma(A)} \lambda \rho(x) d \mu_{F}, \rho(x) \geq 0$ e integrável. Por tanto, $A$ admite uma representação como um operador autoadjunto num espaço de Hilbert, de tal modo que os estados, atuando nas observáveis, se escrevem da forma:

$$
\omega(A)=\langle\Psi|A| \Psi\rangle=\int_{\sigma(A)} \overline{\Psi(\lambda)} \lambda \Psi(\lambda) d \mu_{F} .
$$

$\mathrm{Na}$ verdade, qualquer outro estado pode ser obtido através limites convergentes do tipo (compare com 5.55):

$$
\omega(A)=\lim _{n \rightarrow \infty} \int_{\sigma(A)} \lambda \rho_{n}(x) d \mu_{F}
$$

O estado $\Psi$ poderia ser definido como $\Psi=e^{i \theta(\lambda)} \sqrt{\rho(\lambda)}$, para qualquer $\theta(\lambda)$ integrável. Medidas de integração permitem tratar um espectro contínuo ou discreto no mesmo formalismo.

Uma observável poderia ser definida como um conjunto de números, resultado da sua medida em todos os estados. Apenas mostramos que podemos sempre reunir esse conjunto de números num operador autoadjunto e essa construção se torna necessária para dar conta de incertezas experimentais na construção de estados, ou a formulação estatística da mecânica.

Por tanto, o formalismo anterior nos diz que podemos representar $A$ como operador autoadjunto num espaço de Hilbert. Idem para qualquer outra observável $B \in \mathcal{O}$, que deve atuar no mesmo conjunto de estados. Assim sendo, podemos sempre definir um conjunto de observáveis como um conjunto de operadores no espaço de Hilbert, definindo a sua soma e produto a partir das suas respectivas representações no espaço de Hilbert. O objetivo da definição dessa estrutura algébrica seria recuperar a representação dos operadores a partir da informação algébrica. Comparando o formalismo quântico usual o com que estamos definindo aqui, observamos que a medida de uma observável $A$ num estado 6.8 é na verdade o valor esperado, que é determinístico, e essa definição é conveniente, pois, por erros intrísecos ao processo de medida que gostaríamos de excluir da definição da medida, é conveniente definir o resultado de uma medida, não como o resultado de uma experiência 
particular, mas sim de uma média de um número arbitrariamente grande de repetições do experimento.

Observe que o formalismo desenvolvido dá ainda uma nova interpretação para o $\boldsymbol{a u}$ toestado generalizado 5.55, um funcional multiplicativo da $C^{*}$-álgebra gerada por $A=A^{*}$. Observe ainda que 6.8 coloca uma observável $A$ numa forma que só existe um autoestado generalizado linearmente independente para cada autovalor. De fato, não mais que isso seria necessário se só existisse uma observável na teoria, pois não teríamos condições de distinguir autoestados generalizados diferentes, a não ser se produzissem valores diferentes para alguma outra observável. Mas poderíamos gerar outras representações da observável $A$, na qual a mesma é o operador multiplicação $\theta(\lambda) \neq \lambda$ real, definida no compacto $X$, cujo espectro seria o conjunto dos pontos $\lambda$ tal que $(\theta(\lambda)-\lambda)$ não possui inversa, ou seja, é igual a 0. Essa função poderia ter máximos e mínimos de modo a ter mais que um autoestado generalizado para cada $\lambda$. Toda essa discussão nos mostra um possível generalização da $C^{*}$-álgebra: a regra de multiplicação das observáveis poderia, assim com a multiplicação de operadores lineares num espaço de Hilbert, ser não comutativa. Definimos então a $C^{*}$-álgebra não comutativa, pela mesma definição, mas, cujo produto associativo é não comutativo.

Em resumo, $C^{*}$-álgebra comutativa faz corresponder um único espaço topológico compacto. A correspondência entre espaço e álgebra foi explicitada. Diferentemente da abordagem convencional da geometria na qual definimos primeiro o espaço e depois funções no espaço, tal como no capítulo 2, podemos primeiro definir as funções e o espaço estaria implicitamente definido. No caso não comutativo, não mais podemos encontrar um espaço topológico $X$ associado a $C^{*}$-álgebra, tendo então o espaço nãa comutativo. Conforme detalharemos adiante, resta ainda uma caracterização que é válida tanto no caso comutativo, como não comutativo: a interpretação dos elementos da $C^{*}$-álgebra como operadores limitados no espaço de Hilbert.

A ideia de que o espaço-tempo deveria ser substituída por variáveis não comutativas remota a Heisenberg numa tentativa de regularizar integrais divergentes na Teoria de Campos Snyder (1947). O sucesso do programa de renormalização descartou essa ideia. Contudo, desenvolvimentos na teoria de cordas e teoria $M$ sugerem que o espaço-tempo não comutativo deveria exercer papel proeminente na física da escala de Planck Connes 
et al. (1998). Mais ainda, heuristicamente, poderíamos esperar que a teoria final da gravidade quântica deveria incorporar algum tipo de princípio de incerteza entre coordenadas espaciais Doplicher et al. (1995), uma vez que localizar uma partícula arbitrariamente no espaço, de acordo com a mecânica quântica, requer experimentos com partículas de energias arbitrariamente altas, que, de acordo com a relatividade geral, poderia criar um horizonte de eventos sobre a medida, o que a invalidaria.

Uma vez entendido que a formulação de $C^{*}$-álgebra das observáveis físicas é uma liberdade de formulação, análoga à liberdade de formulação tensorial da física, o grande salto conceitual da mecânica quântica é a constatação de Heisenberg de que a álgebra de observáveis é não comutativa 3 .

Como se daria a dinâmica no caso de uma $C^{*}$-álgebra não comutativa? Como no caso comutativo, um *-automorfismo contínuo de um parâmetro $t$. Esse requerimento diz tão somente que a medida de observáveis em estados muda continuamente com o tempo e que observáveis são mapeadas em observáveis.

Temos então que, da condição de que estados continuam normalizados durante a evolução, $\left\langle\Psi\left|\alpha_{t} 1\right| \Psi\right\rangle=\langle\Psi|1| \Psi\rangle$ para todo $\Psi$. Que pode ser reescrita da forma

$$
\left\langle\Psi\left|\alpha_{t} 1\right| \Psi\right\rangle=\left\langle\Psi\left|U^{*}(t) U(t)\right| \Psi\right\rangle
$$

visto que a regra de transformação das observáveis se traduz na regra de transformação dos estados. Somando a isso o fato de que a transformação pode ser invertida, $U(t)$ é unitário. A continuidade fraca e a propriedade $\alpha_{t} \circ \alpha_{t^{\prime}}=\alpha_{t+t^{\prime}}$, implicam, pelo o teorema de Stone 3 , que $U(t)=e^{-i A t}$ :

$$
\left\langle\Psi\left|\alpha_{t} A\right| \Psi\right\rangle=\left\langle\Psi\left|e^{i H t} A e^{-i H t}\right| \Psi\right\rangle
$$

Assim sendo, numa $C^{*}$-álgebra não comutativa, todo *-automorfismo define uma observável e vice versa. Podemos assumir a evolução diferenciável, ao invés de simplesmente contínua (visto que a diferenciabilidade da dinâmica provavelmente não seria experimen-

\footnotetext{
${ }^{3}$ Nesse ponto, essa afirmação tem, a princípio, um significado físico bem definido: determine o operador autoadjunto correspondente a cada observável, medindo o resultado da observação em cada estado possível, e verifique a regra de multiplicação entre estes, visto que estes operadores atuam no mesmo espaço de estados.
} 
talmente determinável). Nesse caso:

$$
\frac{d}{d t} \omega\left(\alpha_{t} A\right)=-i \omega([H, A])
$$

Observe que se a álgebra $\mathcal{A}$ é comutativa, 6.11 ainda sim é válida, uma vez que 6.3 é uma transformação unitária, mas se $H \in \mathcal{A}_{0}$, 6.11 é trivialmente constante. Por tanto, $H \notin \mathcal{A}$. Mas podemos escrever a evolução das álgebras comutativas de um modo que envolve apenas observáveis da álgebra:

$$
\frac{d}{d t} \omega\left(\alpha_{t} A\right)=\omega(\{H, A\})
$$

que também exprime a dualidade entre possíveis dinâmicas e observáveis.

Assumindo a não comutatividade, se deduzirmos a regra de multiplicação, a matemática que desenvolveremos adiante nos dirá quais são os operadores correspondentes às observáveis, a menos de uma questão de unicidade. A física clássica se baseia na comutatividade da álgebra de observáveis, enquanto o paradigma quântico na não comutatividade. Isso sugere que, em certo limite, a teoria não comutativa se comporta como uma teoria comutativa. Tentemos determinar a teoria não comutativa que reproduza o comportamento clássico. Suponhamos o seguinte requerimento: Suponha $\mathcal{A}_{\hbar}$ uma família de um parâmetro de $C^{*}$-álgebras, parâmetro que chamaremos convenientemente de $\hbar, \hbar \geq 0$. Para cada $\hbar$ e cada elemento $A_{0} \in \mathcal{A}_{0}$, existe uma correspondência que mapeia $A_{0}$ em um elemento $A_{\hbar} \in \mathcal{A}_{\hbar}$. Do mesmo modo, para cada estado $\omega_{0}$ de $\mathcal{A}_{0}$ existe uma correspondência que o mapeia no estado $\omega_{\hbar} \in \mathcal{A}_{\hbar}$. Ambas as correspondências podem ser consideradas sobrejetivas, o que significaria dizer que cada observável e estado quânticos tem um análogo clássico no limite $\hbar \rightarrow 0$. Essa família aproxima a teoria clássica da seguinte maneira:

$$
\lim _{\hbar \rightarrow 0} \omega_{\hbar}\left(\left[A_{\hbar}, B_{\hbar}\right]\right)=0
$$

e dada a correspondência entre dinâmicas e observáveis, estabeleçamos a regra:

$$
\lim _{\hbar \rightarrow 0} \omega_{\hbar}\left(\alpha_{t}^{(\hbar)} A_{\hbar}\right)=\omega_{0}\left(\alpha_{t}^{(0)} A_{0}\right)
$$

para todo estado $\omega_{0}$ na álgebra clássica comutativa. Observe que, sendo $\alpha_{0}^{(0),(h)}=I$, o que pode ser assumido sem perda de generalidade, 6.15 implica que:

$$
\lim _{\hbar \rightarrow 0} \omega_{\hbar}\left(A_{\hbar}\right)=\omega_{0}\left(A_{0}\right)
$$


Chamaremos a regra $A_{0} \rightarrow A_{\hbar}$ de quantização.

A condição 6.15 nos leva a 4

$$
\lim _{\hbar \rightarrow 0} \frac{d}{d t} \omega_{\hbar}\left(\alpha_{t}^{(\hbar)} A_{\hbar}\right)=\frac{d}{d t} \omega\left(\alpha_{t}^{(0)} A_{0}\right)
$$

que nos leva a:

$$
\begin{aligned}
\lim _{\hbar \rightarrow 0} \omega_{\hbar}\left(-i\left[H_{\hbar}, A_{\hbar}\right]\right)-\omega_{0}\left(\left\{H_{0}, A_{0}\right\}\right) & =0 \\
\lim _{\hbar \rightarrow 0} \omega_{\hbar}\left(-i\left[H_{\hbar}, A_{\hbar}\right]-\left\{H_{0}, A_{0}\right\}_{\hbar}\right) & =0,
\end{aligned}
$$

onde empregamos 6.16

Mas vemos aqui que 6.14 implicaria que $\omega_{0}\left(\left\{H_{0}, A_{0}\right\}\right)=0$ para todo $\omega_{0}$, que trivialmente implicaria que todos os elementos de $\mathcal{A}_{0}$ são nulos. Podemos, contudo, sem perda de generalidade, assumir que a dinâmica clássica gerada por $H_{0}$ é aproximada pela dinâmica quântica gerada por $\frac{H_{\hbar}}{\hbar}$, que implica então em:

$$
\lim _{\hbar \rightarrow 0} \omega_{\hbar}\left(\frac{\left[H_{\hbar}, A_{\hbar}\right]}{i \hbar}-\left\{H_{0}, A_{0}\right\}_{\hbar}\right)=0
$$

para todo $\omega_{h}$, que implica em:

$$
\lim _{\hbar \rightarrow 0}\left(\frac{\left[H_{\hbar}, A_{\hbar}\right]}{i \hbar}-\left\{H_{0}, A_{0}\right\}_{\hbar}\right)=0
$$

Essa é justamente a prescrição de Dirac para a quantização (princípio da correspondência):

$$
\left[f_{\hbar}, g_{\hbar}\right]=i \hbar\{f, g\}_{\hbar}+\mathcal{O}\left(\hbar^{2}\right)
$$

\subsection{Teoria quântica sob hipótese de espaço-tempo quântico}

Observe que, em toda a dedução, $\hbar$ era um parâmetro adimensional, o que fisicamente corresponderia a medir $\hbar$ em alguma unidade conveniente com dimensão de ação. Observe ainda que chegamos a esse resultado sem considerar o princípio da incerteza de Heisenberg, assumindo apenas que existe uma $C^{*}$-álgebra não comutativa que aproxima

\footnotetext{
${ }^{4}$ Observe que, matematicamente, o limite 6.15 não implica que o mesmo é válido para as derivadas, a menos que exista uma convergência uniforme das derivadas. Mas, ao introduzir uma topologia no espaço das dinâmicas, devemos assumir que, possivelmente, o grau de diferenciabilidade não é uma observável física, de modo que podemos assumir convergência como aquela das funções teste.
} 
(no sentido considerado) uma $C^{*}$-álgebra comutativa. Isso justifica o procedimento de quantização canônica ser aplicado também para campos, conforme o aplicado na teoria de perturbações cosmológicas na subseção 4.4 .2 do capítulo 4 , ainda que sem respaldo experimental semelhante àquele do princípio da incerteza. Na prescrição original de Dirac, $\mathcal{O}\left(\hbar^{2}\right)$ era identicamente nulo, o que, conforme já argumentado na referida subseção, é provado não poder ser satisfeito. Observe que 6.21 não determina completamente a regra de quantização, desse modo, justifica também deformações das mesmas regras de quantização canônicas, como a adotada em Carmona et al. (2003) para quantização do campo escalar relativístico complexo $\Phi(x)=\Phi_{1}(x)+i \Phi_{2}(x)$ :

$$
\begin{gathered}
{\left[\Phi_{i}(x), \Phi_{j}(y)\right]_{T . I .}=i \epsilon_{i j} \bar{\Theta} \delta(x-y)} \\
{\left[\Pi_{i}(x), \Pi_{j}(y)\right]_{T . I .}=0} \\
{\left[\Pi_{i}(x), \Phi_{j}(y)\right]_{\text {T.I. }}=i \delta_{i j} \hbar \delta(x-y),}
\end{gathered}
$$

onde $\epsilon_{i j}$ é o símbolo de Levi-Civita totalmente antissimétrico, T.I. significa que o comutador é tomado em tempos iguais, $\Phi_{i}, i=1,2$ são os componentes do campo complexo bosônico livre, $\Pi_{i}$ seus respectivos momentos canonicamente conjugados e o termo $\bar{\Theta}$ é assumido ser muito menor que $\hbar$, de modo que leva a uma modificação na teoria de campos que se manifesta numa escala de ações (dimensão de $\hbar$ ) muito menor que $\hbar$. Assim sendo, podemos reescrever sem perda de generalidade: $\bar{\Theta}=\hbar^{2} \Theta$. Essa prescrição, embora produza a teoria clássica de campos no limite $\hbar \rightarrow 0$, produz uma teoria quântica não relativística.

Até então, em nossa análise, todas as observáveis têm sido representadas por operadores limitados. Essa restrição significa que a medida de uma observável é limitada, ou seja, produz um resultado máximo finito. E quanto a observáveis como posição e momento, que a princípio podem assumir valores ilimitados? Na descrição Hamiltoniana, restrição análoga se manifesta na restrição do espaço de fases a uma região compacta, que pode então ser recuperada algebricamente. Essa restrição não é um problema se a Hamiltoniana considerada restringe a dinâmica a essa região (todas as Hamiltonianas consideradas obtidas por considerações algébricas como as discutidas têm essa propriedade). Como remover essas restrições?

Para recuperar todo o espaço de fase e não apenas uma porção compacta dele, podemos recorrer a uma construção que decorre da observação de que um espaço não compacto, tal 
como $\mathbb{R}^{N}$, é a união de uma coleção infinita de compactos $X_{n}, n=1,2,3 \cdots$. Podemos assumir $X_{i} \subseteq X_{j}, i \leq j$. A descrição algébrica de $\mathbb{R}^{N}$ deve estar contida na álgebra das funções complexas em $\mathbb{R}^{N}, C\left(\mathbb{R}^{N}\right)$, mas precisamos dar uma descrição algébrica para estas que nos permita utilizar o formalismo anterior, o qual só permite recuperar espaços compactos. Podemos considerar $C\left(\mathbb{R}^{N}\right)$ uma união de $C^{*}$-álgebras $\mathcal{A}_{i}$, tal que $\mathcal{A}_{i} \subseteq \mathcal{A}_{j}$, ou seja,

$$
\mathcal{A}=\bigcup_{i} \mathcal{A}_{i}
$$

Aqui, $\mathcal{A}_{j}$ estende $\mathcal{A}_{i}$ do seguinte modo: admitamos que existe um identificação entre cada elemento $A_{i} \in \mathcal{A}_{i}$ e algum elemento $A_{j} \in \mathcal{A}_{j}, j \geq i$, tal que para cada estado $\omega_{i}$ de $\mathcal{A}_{i}$ existe um estado $\omega_{j}$ de $\mathcal{A}_{j}$, tal que $\omega_{i}\left(A_{i}\right)=\omega_{j}\left(A_{j}\right)$. Para tanto, basta que exista um único estado $\omega_{i}^{F}$ fiel, isto é, $\omega_{i}^{F}\left(A^{*} A\right)>0$ para todo $A \neq 0, A \in \mathcal{A}_{i}$, que faça corresponder um estado $\omega_{j}^{N F}$ não fiel em $\mathcal{A}_{j}$ tal que $\omega_{j}^{N F}\left(A_{j}\right)=\omega_{i}^{F}\left(A_{i}\right)$. Nesse sentido, cada um dos $\mathcal{A}_{i}$ representa as funções contínuas no compacto $X_{i} \subset \mathbb{R}^{N}$. No mesmo sentido, estendemos a noção de observável de modo a remover a restrição de um limite superior da medição, identificando certas construções experimentais como extensões de outras, ou a álgebra gerada por elas, como extensões da álgebra gerada por outras. Nesse sentido, embora cada construção experimental tenha o seu limite intrínseco (superior e inferior) de medição de uma dada observável, tal como a posição, a princípio, é possível melhorar a construção de modo a aumentar o limite superior e/ou diminuir o limite inferior. Assim sendo, tais observáveis, como posição e momento, ainda têm a interpretação de operadores autoadjuntos, só que ilimitados. De modo equivalente, podemos definir a $C^{*}$-álgebra de maneira idêntica, mas trocando uma única norma por uma família infinita enumerável de seminormas ${ }^{5}\|\cdot\|_{i}$, $i=1,2, \cdots$ tais que $\|A\|_{j} \geq\|A\|_{i}$ se $j \geq i$.

Assim sendo, o princípio da incerteza de Heisenberg:

$$
\left(\Delta_{\omega} q\right)\left(\Delta_{\omega} p\right) \geq \frac{\hbar}{2}
$$

onde $\left(\Delta_{\omega} A\right)^{2}=\omega\left((A-\omega(A))^{2}\right)$, considerando o seguinte resultado para operadores autoadjuntos:

$$
\left(\Delta_{\omega} A\right)\left(\Delta_{\omega} B\right) \geq \frac{1}{2}|\omega([A, B])|
$$

\footnotetext{
${ }^{5}$ Satisfaz todas as propriedades da norma, exceto $\|A\|=0 \Leftrightarrow A=0$.
} 
e assumindo que 6.24 é independente do estado $\omega$, implica que $[A, B]$ é múltiplo da identidade. Sendo $q$ e $p$ autoadjuntos:

$$
\left[q_{i}, p_{j}\right]=i \hbar \delta_{i j}
$$

A consideração de operadores ilimitados para realização do princípio da incerteza é inevitável, pois o mesmo não poderia ser implementado sem a hipótese de pelo menos um dos operadores envolvidos ser ilimitado.

Para completar o processo de quantização a partir do princípio da incerteza, precisamos gerar a álgebra das observáveis. Uma das possíveis prescrições compatíveis com 6.20 é a chamada quantização de $\boldsymbol{W e y l}$, que é definida pelas relações (aqui, denotamos $\hat{f}$ o operador correspondente à função clássica $f$ ):

$$
\begin{array}{r}
f(q, p)=\frac{1}{(2 \pi)^{\frac{n}{2}}} \int \tilde{f}(\mu, \nu) e^{i(\mu q+\nu p)} d \mu d \nu \\
\hat{f}(\hat{q}, \hat{p})=\frac{1}{(2 \pi)^{\frac{n}{2}}} \int \tilde{f}(\mu, \nu) e^{i(\mu \hat{q}+\nu \hat{p})} d \mu d \nu \\
e^{i(\mu \hat{q}+\nu \hat{p})}=e^{i \frac{\hbar \mu \nu}{2}} e^{i \mu \hat{q}} e^{i \nu \hat{p}}
\end{array}
$$

onde aplicamos a fórmula de Baker-Hausdorff:

$$
e^{A} e^{B}=e^{A+B+\frac{1}{2}[A, B]}, \text { desde que }[[A, B], A]=[[A, B], B]=0
$$

e a álgebra 6.27 é do tipo 6.23, sendo a álgebra $\mathcal{A}_{j}$ obtida fazendo-se $f(q, p)$ um função com suporte em $[-j,+j] \times[-j,+j]$. Chamaremos a regra $\hat{W}: f(q, p) \rightarrow \hat{f}(\hat{q}, \hat{p})$ quantização de Weyl (Veja as propriedades 1 a 5 na subseção 4.4.2).

Nesse ponto, já está claro que um espaço-tempo não comutativo é obtido pela modificação da $C^{*}$-álgebra comutativa das funções contínuas do espaço-tempo de modo a obter uma versão não comutativa. Podemos partir de uma relação tal como $\left[x^{\mu}, x^{\nu}\right]=i \Theta^{\mu \nu} \neq 0$ e obter tal álgebra por um esquema análogo à quantização de Weyl. Como definir a teoria quântica no espaço não comutativo? Podemos postular (ainda em acordo com 6.20):

$$
\begin{aligned}
& {\left[x^{i}, x^{j}\right]=i \hbar^{2} \Theta^{i j},} \\
& {\left[x^{i}, p^{j}\right]=i \hbar \delta^{i j}}
\end{aligned}
$$

após o que, definimos a quantização via 6.27 .

Este esquema de quantização deformada é tratado em vários trabalhos, como por exemplo Gamboa et al. (2001), sendo Carmona et al. (2003), definido por 6.22, uma possível 
generalização da proposta para tratar o caso de quantização de campos. No caso 6.29, o teorema de Von Neumann ainda nos permite tratar o problema da sua realização, pois este teorema afirma que, num espaço de Hilbert separável para cada $i$ :

$$
x^{i}=\sum_{N=0}^{\infty}{ }^{\oplus} U_{N}^{-1} x U_{N} \text { e } p^{i}=\sum_{N=0}^{\infty}{ }^{\oplus} U_{N}^{-1} p U_{N},
$$

onde $U_{N}$ é um conjunto de transformações unitárias e $(x, p)$ é um representação irredutíve $]^{6}$ de 6.26 (sendo todas unitariamente equivalentes entre si) 7. Podemos também aplicar 6.30 no caso $\Theta^{i j}$ constante, o que se chama de não comutatividade canônica, que se torna trivial fazendo-se ?:

$$
x_{C}^{i}=x^{i}+\frac{1}{2 \hbar} \theta^{i j} p_{j}
$$

que nos leva a:

$$
\begin{aligned}
& {\left[x_{C}^{i}, x_{C}^{j}\right]=0,} \\
& {\left[x_{C}^{i}, p^{j}\right]=i \hbar \delta^{i j}}
\end{aligned}
$$

Faz parte da definição da teoria que a realização de 6.29 deve ser ser escolhida irredutível (conforme definição na subseção 5.2.3). Observe que isso não implica que, para cada $i,\left(x^{i}, p^{i}\right)$ forma uma representação irredutível da álgebra de Heisenberg, por isso a representação redutível 6.30.

Uma vez que o campo é o ingrediente essencial na implementação do requerimento da localidade em física, conforme discussão no capítulo 2, é desejável definir a quantização de campos no espaço-tempo não comutativo, uma teoria que, no limite semiclássico, comportese como uma teoria local. A filosofia dos espaços não comutativos diz que apenas conceitos algébricos formulados em termos da $C^{*}$-álgebra comutativa têm a chance de serem generalizados para o caso não comutativo. Felizmente, a teoria quântica de campos é construída a partir da álgebra dos campos clássicos. Substituímos, então, essa álgebra por aquela gerada pela quantização de Weyl 6.27 sob a hipótese $\left[x^{\mu}, x^{\nu}\right] \neq 0$. Esta álgebra pode ser representada como uma álgebra de operadores no espaço de Hilbert, mas podemos utilizar a quantização de Weyl para definir um isomorfismo entre tais operadores e a álgebra das funções ordinárias no espaço-tempo com um produto modificado não comutativo. Com efeito, 6.27 já é uma correspondência linear e isométrica $\left(\|f(q, p)\|=\sup _{K}|f(q, p)|=\|\hat{W}(f(q, p))\|\right.$,

\footnotetext{
${ }^{6}$ Veja definição de irredutibilidade em $[5.2 .3$

7 Veja a definição de soma direta no footnote 3 do capítulo 5
} 
em que $\|\hat{W}(f(q, p)) \Psi\| \leq\|\hat{W}(f(q, p))\| \cdot\|\Psi\|)$. Falta apenas tornar a correspondência isomórfica, ou seja, preservar o produto, mas isso é feito modificando-se o produto das funções de tal modo que:

$$
\hat{W}(f) \cdot \hat{W}(g)=\hat{W}(f \star g)
$$

Temos desse modo uma autêntica realização da $C^{*}$-álgebra. A operação ^ é conhecida como produto estrela. O que fazer com essa álgebra? Uma proposta é modificar a ação dos campos Douglas e Nekrasov (2001), dada que uma vertente da teoria de campos assume que toda teoria quântica é determinada pela ação:

$$
S=\int\left(\frac{1}{2} \partial_{\mu} \Phi \partial^{\mu} \Phi-\frac{m^{2}}{2} \Phi \Phi\right) d^{4} x \rightarrow S=\int\left(\frac{1}{2} \partial_{\mu} \Phi \star \partial^{\mu} \Phi-\frac{m^{2}}{2} \Phi \star \Phi\right) d^{4} x
$$

Na expressão acima, tanto a derivada como a integral são expressas através das operações da álgebra $C^{*}$. De fato, é possível mostrar que as derivadas parciais na álgebra $\mathcal{A}$ de funções suaves no compacto $K \subset \mathbb{R}^{n}$, ou mesmo em $\mathbb{R}^{n}$, são unicamente determinadas pelas seguintes propriedades:

1. $\partial_{i}(\alpha A+\beta B)=\alpha \partial_{i}(A)+\beta \partial_{i}(B)$ (linearidade);

2. $\partial_{i}(A \cdot B)=\partial_{i}(A) \cdot B+A \cdot \partial_{i}(B)($ regra de Leibniz);

3. $\partial_{i} x^{j}=\delta_{i}^{j}$

Sendo que 3 pode ser substituída por $\left[\partial_{i}, \partial_{j}\right]=0$, de modo que o teorema de Frobenius (referenciado na vizinhança de 2.30) implica que existe uma mudança de coordenadas na vizinhança $K$ de cada ponto tal que $\partial_{i}$ satisfaz 3 nas novas coordenadas.

A integral é inteiramente codificada pelas propriedades 9 ;

${ }^{8}$ Demonstremos o caso $n=1$, não há complicação adicional no caso geral. Pelo teorema do valor intermediário: $f(x)=f(a)+f^{\prime}(a)(x-a)+\frac{1}{2} f^{\prime \prime}(c)(x-a)^{2}, \quad c \in(a, x)$. Pela linearidade: $(\partial f)(a)=$ $\partial(f(a))(a)+\partial\left(f^{\prime}(a)(x-a)\right)(a)+\partial\left(\frac{1}{2} f^{\prime \prime}(c)(x-a)^{2}\right)(a)$, mas pela regra de leibniz $\partial(c t e)=0$ e $\partial\left(\frac{1}{2} f^{\prime \prime}(c)(x-\right.$ $\left.a)^{2}\right)(a)=2(x-a) \partial\left(\frac{1}{2} f^{\prime \prime}(c)(x-a)\right)$, o que implica que o terceito termo em $(\partial f)(a)$ é zero no ponto $x=a$. Assim sendo $(\partial f)(a)=\left(f^{\prime}(a) \partial(x)\right)(a)$.

${ }^{9}$ Aqui, assumimos que as funções são diferenciáveis e tem suporte compacto $K$, em geral, as funções diferenciáveis da $C^{*}$ álgebra em $K$ não têm essa propriedade, mas podemos estendê-las de maneira diferenciável para um compacto $K^{\prime} \supseteq K$ de modo a satisfazer isso, sendo a medida de $K^{\prime}-K$ arbitrariamente pequena. Demonstremos também no caso $n=1$, assuma $K=[0, \pi]$, o que não é uma restrição, visto que podemos realizar uma mudança de coordenadas de modo que o suporte da função esteja contido em 
1. $\int$ é um operador linear limitado;

2. $\int \partial_{i} A=0$;

3. $\int[A, B]=0$;

mais uma vez demonstrando o poder da álgebra de codificar outros conceitos, podemos simplesmente assumir $\mathcal{A}$ uma $C^{*}$-álgebra comutativa (ou um limite do tipo 6.23) e recuperar o cálculo integral e diferencial. A propriedade 3 é redundante no caso comutativo. Essa propriedade nos diz que a integral é na verdade o traço dos operadores $\mathcal{A}$. De fato, considere a álgebra das matrizes de dimensão $n$ finita, um exemplo de $C^{*}$-álgebra, que denotaremos $M(n)$. Suponha uma operação satisfazendo 1 e 3 , atuando num elemento autoadjunto $A \in M(n)$. Sabemos que existe uma transformação unitária $U \in M(n)$ tal que $A=U^{-1} \operatorname{diag}\left(\lambda_{1}, \cdots, \lambda_{n}\right) U$. Pela propriedade 3, $\int A=\int \operatorname{diag}\left(\lambda_{1}, \cdots, \lambda_{n}\right)$, mas esta é linear por 1, $\operatorname{logo} \int A=\sum_{j} c_{j} \lambda_{j}$. Mas existe outra transformação unitária $U_{2}$ que permuta a ordem dos autovalores, de modo que $\int A=c \sum_{j} \lambda_{j}=c \operatorname{Tr}(A)$. Isso implica que só existe uma integral em dimensão finita, a menos de uma normalização, apesar da propriedade 2. De fato, em dimensão finita, toda derivada é da forma $\partial_{B} A=[B, A]$, de modo que 3 equivale à 2. No caso de uma $C^{*}$-álgebra genérica, dado um elemento autoadjunto, existe uma transformação unitária que o mapeia numa função contínua num compacto, 3 nos diz, por raciocínio análogo, que a integral é um funcional linear contínuo dessa função, onde empregamos o raciocínio do footnote 9. Observe que podemos aproximar uma álgebra de funções contínuas num compacto por uma álgebra de matrizes, colocando os valores $f\left(x_{i}\right)$ como entradas na diagonal, para um conjunto discreto de pontos $x_{i}$, no qual 3 nos daria uma aproximação arbitrariamente boa da integral.

Também na formulação 6.34, a não comutatividade canônica, $\left[x^{\mu}, x^{\nu}\right]=i \Theta^{\mu \nu}$ produz um caso analiticamente tratável. De fato, devido a 6.28 , decorre que $e^{i k_{\mu} x^{\mu}} e^{i q_{\nu} x^{\nu}}=$ $e^{i \frac{1}{2} \theta_{\mu \nu} k^{\mu} q^{\nu}} e^{i\left(k_{\mu}+q_{\mu}\right) x^{\mu}}$, assim sendo 6.33 se exprime alterando-se a regra de convolução:

$$
\int d k \phi(k) \psi(q-k) \rightarrow \int d k \phi(k) \psi(q-k) e^{i \frac{1}{2} \theta_{\mu \nu} k^{\mu}(q-k)^{\nu}}
$$

$[0, \pi]$. Escreva $f(x)=\sum_{n=0}^{\infty} \cos (n x) \int_{0}^{\pi} \frac{2}{\pi} \cos (n t) f(t)$. Observe que $\cos (n x)=n \partial \sin (n x), n \geq 1$, sendo $\sin n x$ uma função de suporte em $K$. Ao considerarmos o fato de que, para $f(x)$ suave e de período $2 \pi,\left\|\sum_{n=M}^{\infty} \cos (n x) \int_{0}^{\pi} \frac{2}{\pi} \cos (n t) f(t)\right\|<\epsilon$ se $M$ for tomado suficientemente grande, e o fato de que $\int f(x) \leq C\|f(x)\|$ (limitado) e a linearidade da integral, decorre que $\int f(x)=\frac{2}{\pi} \int f(t) d t$. 
Decorre que as derivadas usuais fornecem um conjunto comutativo de derivações nessa álgebra:

$$
\begin{aligned}
\partial^{\mu} \phi(x) \star \psi(x) & =\int d^{4} q \frac{1}{(2 \pi)^{2}} e^{-i q x} q^{\mu} \int d k \phi(k) \psi(q-k) e^{i \frac{1}{2} \theta_{\mu \nu} k^{\mu}(q-k)^{\nu}} \\
& =\int d^{4} q \frac{1}{(2 \pi)^{2}} e^{-i q x} \int d k\left(q^{\mu}-k^{\mu}+k^{\mu}\right) \phi(k) \phi(q-k) e^{i \frac{1}{2} \theta_{\mu \nu} k^{\mu}(q-k)^{\nu}} \\
& =\left(\partial^{\mu} \phi(x)\right) \star \psi(x)+\phi(x) \star\left(\partial^{\mu} \psi(x)\right),
\end{aligned}
$$

que nos leva à mesma regra de integração usual e, por consequência:

$$
\int \phi(x) \star \psi(x)=\int d x \int \frac{d^{4} q}{(2 \pi)^{2}} e^{-i k q} \int d k \phi(k) \phi(q-k) e^{i \frac{1}{2} \theta_{\mu \nu} k^{\mu}(q-k)^{\nu}}=\int \phi(x) \psi(x),
$$

onde usamos $\theta_{\mu \nu} k^{\mu} k^{\nu}=0$. Daí concluímos que 6.34 não afeta ações quadráticas nos campos, ou seja, teorias livres.

Nesse momento, poderíamos citar que em última análise 6.34 se manifesta da modificação das regras de Feymann que calculam a matriz de espalhamento da teoria 10 . Existem problemas ao aplicar os métodos usuais de renormalização sob as regras de Feymann modificadas. Discutir todas as implicações da teoria quântica associada a 6.34 está além do escopo desta tese, mas podemos citar que fazer $\Theta^{i 0} \neq 0$ implica numa matriz de espalhamento não unitária Rim e Yee (2003).

Observe que 6.34 é um postulado, uma definição, para qual existem alternativas, não fazendo sentido perguntar se esta é a maneira certa de implementar a ideia de não comutatividade das coordenadas como deformação da teoria relativística de campos usual.

\subsection{O caráter não relativístico de um espaço-tempo quântico}

A ideia de espaço-tempo quântico necessariamente vem acompanhada da violação da relatividade? A resposta a essa pergunta, na verdade, só pode ser postulada, a partir do que se define por formulação da física sob hipótese de espaço-tempo não comutativo. Visto que o processo de quantização não é unicamente determinado, tal formulação pode ser definida como uma deformação do processo de quantização dos sistemas clássicos.

Uma maneira de formular o requerimento da invariância relativística da física seria considerar que as observáveis clássicas $\mathcal{O}$ são de algum modo dependentes do sistema de

\footnotetext{
10 Transformação unitária que leva um estado inicial (num passado assintótico) com um conteúdo de partículas e momentos bem definidos num estado final espalhado.
} 
coordenadas inerciais, de modo que uma transformação do sistema de coordenadas $(\Lambda, a)$ induz uma representação em $\mathcal{O}$. Ou seja, $T_{(\Lambda, a)}: \mathcal{O} \rightarrow \mathcal{O}$. Assim sendo, o requerimento de invariância relativística do processo de quantização deformado (pela hipótese de espaço não comutativo) seria $\mathcal{Q}_{N C}\left(T_{(\Lambda, a)} A\right)=U \mathcal{Q}_{N C}(A) U^{-1}$, onde $U$ é uma transformação unitária 11. Como a álgebra não comutativa é uma quantização da álgebra das funções contínuas no espaço-tempo, a qual pode ser construída a partir de um conjunto de operadores autoadjuntos $x^{\mu}$, via 6.27, e tornada não comutativa pela substituição $x^{\mu} \rightarrow \mathcal{Q}\left(x^{\mu}\right)$, definindo a álgebra $\mathcal{A}_{N C}$. Independente de como a quantização é deformada pela hipótese não comutativa do espaço, tal dependência se traduz assumindo-se $\mathcal{Q}_{N C}$ dependente da álgebra $\mathcal{A}_{N C}$. Nesse sentido, a teoria quantizada será a mesma se a álgebra gerada por $x^{\mu}$ for a mesma que a gerada por $T_{(\Lambda, a)} x^{\nu}$. Em outras palavras:

$$
\begin{gathered}
\Lambda^{\nu}{ }_{\mu} \mathcal{Q}\left(x^{\mu}\right)=U \mathcal{Q}\left(x^{\nu}\right) U^{-1} \\
\mathcal{Q}\left(x^{\mu}\right)+a^{\nu}=U \mathcal{Q}\left(x^{\nu}\right) U^{-1}
\end{gathered}
$$

onde $U$ é uma transformação unitária e $a^{\mu}$ é um quadrivetor constante (algo semelhante a 5.65). Mais geralmente, o lado esquerdo de 6.38 e 6.39 pode ser entendido como parte de uma realização (não necessariamente unitária) de um grupo numa álgebra, tal representação será unitária se a álgebra gerada por $T_{(\Lambda, a)} A$ é a mesma gerada por $A$ (Veja a construção GNS adiante). Desse modo 6.38, por exemplo, se traduz como:

$$
\left[\mathcal{Q}\left(x^{\mu}\right), \mathcal{Q}\left(x^{\nu}\right)\right]=P^{\mu \nu}\left(\mathcal{Q}\left(x^{\mu}\right), A\right) \rightarrow\left[\Lambda^{\nu}{ }_{\mu} \mathcal{Q}\left(x^{\mu}\right), \Lambda^{\nu}{ }_{\mu} \mathcal{Q}\left(x^{\nu}\right)\right]=P^{\mu \nu}\left(\Lambda^{\nu}{ }_{\mu} \mathcal{Q}\left(x^{\mu}\right), T_{(\Lambda)} A\right)
$$

onde $A$ representa quaisquer outras observáveis algebricamente independentes de $\mathcal{Q}\left(x^{\mu}\right)$, onde atua uma representação $T_{(\Lambda)}$ de Lorentz e que participam de sua álgebra e $P^{\mu \nu}$ são funções algébricas dos argumentos.

Foi num trabalho considerado a origem da ideia de espaço-tempo quântico que Snyder Snyder (1947) mostrou um exemplo de espaço-tempo que satisfaz 6.38.

$$
\begin{gathered}
{\left[x^{\mu}, x^{\nu}\right]=i l^{2} \hbar^{-1}\left(x^{\mu} p^{\nu}-x^{\nu} p^{\mu}\right)} \\
{\left[x^{\mu}, p^{\nu}\right]=i \hbar \eta^{\mu \nu}+i l^{2} \hbar^{-1} p^{\mu} p^{\nu}} \\
{\left[p^{\mu}, p^{\nu}\right]=0}
\end{gathered}
$$

\footnotetext{
${ }^{11}$ Mais geralmente, uma simetria quântica, mas já argumentamos no capítulo anterior que $S L(2, C)$ só admite uma realização unitária
} 
sendo $\eta^{\mu \nu}$ a métrica de Minkowski.

A invariância translacional em 6.39 necessita $\left[x^{\mu}, x^{\nu}\right]=P_{1}^{\mu \nu}\left(x^{\mu}, p^{\nu}\right)$ e $\left[x^{\mu}, p^{\nu}\right]=P_{2}^{\mu \nu}\left(x^{\mu}, p^{\nu}\right)$ independentes de $x^{\mu} 12$, enquanto que, assumindo isso, 6.38 implica 13 ;

$$
\left[x^{\mu}, x^{\nu}\right]=c_{1} p^{\mu} p^{\nu} P_{1}\left(p^{2}\right)+c_{2} \eta^{\mu \nu} P_{2}\left(p^{2}\right)
$$

sendo $P_{1}$ e $P_{2}$ polinômios no quadrado de Minkowski do quadrimomento. Mas o lado esquerdo de 6.42 só pode ser tornado antissimétrico para $c_{1}=c_{2}=0$. Nesse sentido, não existe espaço não comutativo relativisticamente invariante construído a partir de momento e posição. Em particular, não existe álgebra não comutativa gerada apenas por posição que seja relativisticamente invariante. Mas, em última análise, o que determina que a física resultante da consideração de espaço não comutativo é relativisticamente invariante é a maneira como as observáveis quantizadas dependem da álgebra das coordenadas. Por exemplo, num universo povoado por campos livres, 6.34 sob não comutatividade canônica produz uma física totalmente relativística.

Propriedade análoga a 6.38 e 6.39 expressa a invariância relativística da teoria de campos, a qual é definida pelo conjunto de operadores $\int d^{4} x \phi_{i}(x) f_{i}(x) \equiv \phi_{i}\left(f_{i}\right), f_{i}(x)$ uma função de rápido decrescimento nas coordenadas do espaço-tempo para cada $i=1,2 \cdots N$, como argumentado na subseção 4.4.2:

$$
\phi_{i}\left(V_{j i}(A) f_{i}(\Lambda x+a)\right)=U(A, a) \phi_{j}(f(x)) U^{-1}(A, a),
$$

onde $V_{j i}$ é uma representação de dimensão finita de $A \in S L(2, \mathbb{C})$ que corresponde à transformação de Lorentz $\Lambda$. De fato, a quantização canônica do campo escalar relativístico $\left(V_{j i}=I\right)$, pode ser escrita apenas em termos dos operadores $\Phi(x)$ :

$$
[\Phi(x), \Phi(y)]=i \Delta(x-y),
$$

\footnotetext{
${ }^{12} P_{1}^{\mu \nu}\left(x^{\mu}+a^{\mu}, p^{\nu}\right)-P_{1}^{\mu \nu}\left(x^{\mu}, p^{\nu}\right)$, a menos que $P_{1}^{\mu \nu}$ não dependa de $x^{\mu}$, é um polinômio em $a^{\mu}, x^{\nu}$ e $p^{\nu}$ identicamente nulo, mas não existe combinação linear de potências de $x^{\nu}, p^{\nu}$ identicamente nula a não ser que todos os coeficientes sejam nulos (independência linear).

${ }^{13}\left[x^{\mu}, x^{\nu}\right]=c^{\mu \nu}+c_{\alpha}^{\mu \nu} p^{\alpha}+c_{\alpha \beta}^{\mu \nu} p^{\alpha} p^{\beta}+\cdots$. O requerimento 6.40 implica $\left[\Lambda^{\mu^{\prime}}{ }_{\mu} x^{\mu}, \Lambda^{\nu^{\prime}}{ }_{\nu} x^{\nu}\right]=$ $c^{\mu^{\prime} \nu^{\prime}}+c_{\alpha^{\prime}}^{\mu^{\prime} \nu^{\prime}}\left(\Lambda^{\alpha^{\prime}}{ }_{\alpha} p^{\alpha}\right)+c_{\alpha^{\prime} \beta^{\prime}}^{\mu^{\prime} \nu^{\prime}}\left(\Lambda^{\alpha^{\prime}}{ }_{\alpha} p^{\alpha}\right)\left(\Lambda^{\beta^{\prime}}{ }_{\beta} p^{\beta}\right)+\cdots$, que implica em uma identidade do tipo

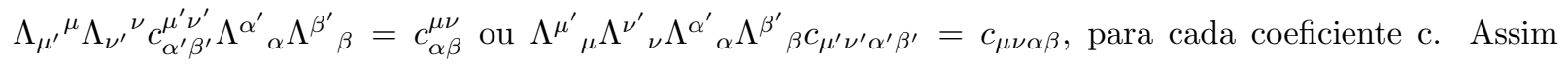
sendo, $c_{\mu \nu \alpha_{1} \alpha_{2} \cdots \alpha_{n}}=c_{i} \sum_{P_{i}} \prod_{(\alpha \beta) \in P_{i}} \eta_{\alpha \beta}$, onde $\eta$ é a métrica de Minkowski e $P_{i}$ é uma partição do conjunto de índices $\mu \nu \alpha_{1} \alpha_{2} \cdots \alpha_{n}$ em pares dijuntos
} 
onde $\Delta(x-y)$ é uma função relativisticamente invariante que se anula quando $z=x-y$ é tipo espaço, o que satisfaz $6.4 \|^{14}$.

Analogamente, pelo artifício do operador de campo, podemos definir a teoria quântica não relativística:

$$
\phi_{i}\left(V_{j i}(g) f_{i}\left(T_{g}(x)\right)\right)=U(g) \phi_{j}(x) U^{-1}(g) \text {, }
$$

onde $V_{j i}(g)$ é uma realização de dimensão finita do elemento $g$ grupo $\mathcal{G}_{P}$, enquanto $T_{g}(x)$ é uma realização, possivelmente não linear, no espaço-tempo.

Mais geral que isso é:

$$
\phi_{i}\left(V_{j i}(g) T_{g} f_{i}(x)\right)=U(g) \phi_{j}(x) U^{-1}(g)
$$

onde $T_{g}$ é uma realização do grupo $\mathcal{G}_{P}$ no espaço de funções teste.

Uma vez que argumentamos que a matéria ser descrita por uma teoria quântica é uma liberdade de formulação, ficando a encargo do experimento verificar se a álgebra de observáveis é comutativa ou não, a hipótese fundamental desta tese é que a matéria é descrita por uma teoria quântica relativística apenas a baixas energias, a qual também é local, tal localidade sendo expressa via o conceito de campo através do requerimento 5.105 , enquanto que 6.43 se transforma segundo 6.45 a altas energias, podendo ou não satisfazer 5.105. Esse fenômeno pode acarretar na inflação como consequência natural das leis físicas derradeiras desde que o grupo $\mathcal{G}_{P}$ seja escolhido apropriadamente. Grupos não isomórficos produzem teorias físicas inequivalentes, uma vantagem sobre outras formulações tais como 6.34, que podem efetivamente não produzir mudança nenhuma na teoria, uma vez que lagrangianas diferentes podem produzir a mesma física. Contemplemos o papel da relação de dispersão da definição de $\mathcal{G}_{P}$.

Inicialmente, precisamos de hipóteses sobre a estrutura do grupo, pois considerar o grupo mais geral possível não apenas é uma tarefa mais complexa, como pode não permitir uma formulação consistente da física. Assumiremos que:

1. $\mathcal{G}_{P}$ é um grupo gerado por um coleção finita de subgrupos comutativos de um parâmetro $P_{i}\left(\theta_{i}\right), i=1, \cdots, N$, ou seja, todo elemento de $\mathcal{G}_{P}$ é obtido pelo produto de elementos desses subgrupos. Destes, os subgrupos $P_{j}\left(\theta_{j}\right), j=1,2,3,4$, geram um grupo comutativo que denotaremos $\mathcal{T}$.

\footnotetext{
${ }^{14} \mathrm{Na}$ verdade, solução da equação de Klein-Gordon 5.73 satisfazendo $\left.\Delta(z)\right|_{z^{0}}=0 ;\left.\frac{\partial \Delta}{\partial z^{0}}\right|_{z^{0}}=-\delta^{3}(z)$
} 
2. Cada um dos subgrupos $P_{i}\left(\theta_{i}\right)$ de $\mathcal{G}_{P}$ é representado de modo regular no espaçotempo, ou seja, $T_{P_{j}\left(\theta_{j}\right)}\left(x^{\mu}\right)$, em 6.45, corresponde a uma trajetória contínua/diferenciável no espaço-tempo para todo $x^{\mu}$, sendo $\mathcal{T}$ realizado como as usuais translações no espaço-tempo.

3. Os elementos $P_{j}\left(\theta_{j}\right), j=4, \cdots N$ formam um subgrupo $\mathcal{L}_{\mathcal{N}} \subset \mathcal{G}_{P}$ cuja representação no espaço-tempo deixa invariante a origem do sistema de coordenadas, $x=0$.

4. A medida de integração $d^{4} x$ é deixada invariante pela ação de $\mathcal{G}_{P}$. Esta é (a menos de um fator multiplicativo) a única medida de integração invariante pelo grupo de translações. Esta propriedade implica que existe uma medida de integração invariante pela ação do grupo, em termos da qual é possível escrever um princípio variacional que seria o mesmo em qualquer um dos sistemas de coordenadas equivalentes. Também assegura que existem representações unitárias do grupo no espaço de Hilbert (veja 6.61 abaixo), um requerimento essencial para a existência da formulação quântica. Dada esta realização, pelo argumento na vizinhança de 5.43 , cada $P_{i}\left(\theta_{i}\right)$ é um subgrupo unitário, assim sendo, $\mathcal{G}_{P}$ o é, e pelo teorema de Stone 3 cada $P_{i}\left(\theta_{i}\right)$ se realiza como $U_{i}\left(\theta_{i}\right)=e^{-i X_{i} \theta_{i}}$, onde $X_{i}$ é um operador autoadjunto definido no domínio $D\left(X_{i}\right)=\left\{\Psi \in \mathcal{H} ; U_{i}\left(\theta_{i}\right) \Psi\right.$ é diferenciável $\}$, obtido do limite:

$$
\lim _{\theta_{j} \rightarrow 0} \frac{U_{j}\left(\theta_{j}\right)-1}{\theta_{j}} \Psi=-i X_{i} \Psi, \quad \Psi \in D\left(X_{i}\right) .
$$

O domínio $D\left(X_{i}\right)$ resulta ser igual ao conjunto das funções $f(x)$ diferenciáveis tais que $\int d x f^{*}(x) f(x) \leq 0$. Esse domínio, contudo, não é invariante pela ação de $X_{i}$, de modo que possamos aplicar livremente potências de $X_{i}$ formando uma álgebra. Tal domínio pode ser obtido restringindo-se à classe das funções $f(x) \in \mathcal{C}^{\infty}, \int d x f^{*}(x) f(x) \leq 0$, domínio no qual $X_{i}$ não é autoadjunto, mas sim essencialmente autoadjunto.

5. No domínio, $\mathcal{D}=\left\{\psi \in \mathcal{H} ; \phi(x) \in \mathcal{C}^{\infty}\right\}$, os geradores formam uma álgebra não trivial: $\left[X_{i}, X_{j}\right]=i C\left(X_{k}\right), C\left(X_{k}\right)$ uma função analítica dos geradores não trivialmente satisfeita por qualquer conjunto $X_{i}$.

6. A álgebra formada pelos geradores das translações, $\mathfrak{t}$, é um $\boldsymbol{i d e a l}$, isto é, seja $\mathfrak{p}_{\mathcal{N}}$ a álgebra formada pelos geradores dos subgrupos $P_{i}\left(\theta_{i}\right)$ no domínio $\mathcal{D},\left[\mathfrak{p}_{\mathcal{N}}, \mathfrak{t}\right] \subseteq \mathfrak{t}$. Esta condição assegura que um estado estacionário num dado referencial, isto é, 
autoestado da energia, é também estacionário quando visto de outro referencial, ou seja, também um autoestado da energia 15 .

7. Os geradores dos subgrupos $P_{i}\left(\theta_{i}\right)$ formam uma álgebra que contém um Casimir obtido como função $C(E, P)$ dos geradores das translações $E$ e $P$ (Sendo uma álgebra comutativa, o teorema Gelfand-Naimark implica numa representação funcional). Assumiremos que as soluções da equação $C(E, P)=\lambda$ definem superfícies nas variáveis $(E, P)$ tais que para algum subconjunto $M_{\mathcal{G}_{P}} \operatorname{dos} \lambda, C(E, P)=\lambda$ contem um componente conexo satisfazendo $E \geq 0$ e $E=0 \Leftrightarrow P^{\mu}=0$, a deformação da concha de massa, $\mathcal{C}_{\lambda}$. O conjunto dos $(E, P)$ tais que $C(E, P) \in M_{\mathcal{G}_{P}}$ e $E \geq 0$ denotaremos como a deformação do cone de luz do futuro $V_{\mathcal{G}_{P}}^{+}$(e.g. comentários em torno de 2.11). Esta propriedade assegura que existam representações irredutíveis do grupo no espaço de Hilbert com energia positivo-definida, ou seja, $\theta(E)$ é um Casimir $\left(\theta\left(p^{0}\right)=1\right.$ se $p^{0} \geq 0$ e 0 em caso contrário) ${ }^{16}$.

A propriedade 6.45, mais as hipóteses estruturais do grupo, implicam em propriedades estruturais na função de $N$-pontos, o valor esperado no vácuo do produto de $N$ operadores de campo. Na argumentação que se segue, vamos assumir a representação do grupo $\mathcal{G}_{P}$ é tal que os geradores realizam a mesma álgebra $\mathfrak{p}_{\mathcal{N}}$, como discutiremos no próximo capítulo,

15 Suponha $\Psi$ uma autoestado da energia, decorre da comutatividade dos geradores de translação espacial e temporal que também é um autoestado do momento. Podemos denotar $P^{\mu} \Psi=p^{\mu} \Psi$, ou, equivalentemente, $e^{i P^{\mu}} \Psi=e^{i p^{\mu}} \Psi$. Dada uma mudança de referencial $e^{i P^{\mu}} \rightarrow U e^{i P^{\mu}} U^{-1}=e^{i C} e^{i P^{\mu}}$, $U e^{i P^{\mu}}=e^{i C} e^{i P^{\mu}} U \Rightarrow e^{i C}=U e^{i P^{\mu}} U^{-1} e^{-i P^{\mu}}$. Podemos supor $U=e^{i X_{i}}$, quando então aplicamos a forma integral da fórmula de Baker-Hausdorff $e^{C}=e^{A} e^{B}$ :

$$
C=B+\int_{0}^{1} g\left(e^{t a d A} e^{a d B}\right) A d t ; \quad g=\ln (z) /(z-1),
$$

onde $a d A$ é a ação adjunta $\operatorname{ad} A(B)=[A, B]$, donde concluímos que $C \in \mathfrak{t}$, decorre ainda que $e^{i C} e^{i P^{\mu}}=$ $e^{i \bar{P}^{\mu}}$, onde $\bar{P}^{\mu}$ é uma função analítica dos momentos $P^{\mu}$ que define uma representação.

${ }^{16}$ Pois as medições de quadrimomento em tais representações produzem valores esperados $\omega\left(P^{\mu}\right)$ que pertencem ao fechamento linear convexo de $\mathcal{C}_{\lambda}$, que denotaremos $\overline{\mathcal{C}_{\lambda c}}$, o conjunto de todas as combinações lineares do tipo $\lambda p_{1}^{\mu}+(1-\lambda) p_{2}^{\mu}, p_{1,2}^{\mu} \in \mathcal{C}_{\lambda}, \lambda \in[0,1]$. Sendo a representação do grupo regular, a ação dos subgrupos gerados por $X_{i}$ nos estados, $\omega_{t}\left(P^{\mu}\right)=\omega\left(e^{i X t} P^{\mu} e^{-i X t}\right)$, produz uma trajetória contínua em $\overline{\mathcal{C}_{\lambda c}}$ que só pode passar para a região $E \leq 0$ pelo ponto $p^{\mu}=0$, equivalentemente pelo estado $\omega_{t_{0}}\left(P^{\mu}\right)=0,\left.\operatorname{mas} \frac{d}{d t} \omega_{t}\left(P^{\mu}\right)\right|_{t=t_{0}}=\omega\left(\left[X_{t_{0}}, P^{\mu}\right]\right)=\omega\left(H\left(P^{\mu}\right)\right)=0, X_{t_{0}}=e^{i X t_{0}} X e^{-i X t_{0}}$, onde utilizamos a propriedade 6 . 
a menos que $\mathcal{G}_{P}$ seja um grupo de Lie, não podemos assegurar que essa propriedade valha em geral.

A primeira propriedade importante deriva da preservação, por hipótese, da invariância translacional da teoria em 2, a função de $N$-pontos depende apenas das diferenças entre as coordenadas:

$$
\left\langle 0\left|\Phi_{i_{1}}\left(x_{1}\right) \Phi_{i_{2}}\left(x_{2}\right) \cdots \Phi_{i_{N}}\left(x_{N}\right)\right| 0\right\rangle \equiv \omega_{\tau}\left(x_{1}, x_{2}, \cdots x_{N}\right)=F_{\tau}\left(\xi_{1}, \xi_{2}, \cdots, \xi_{N-1}\right)
$$

onde $\xi_{i} \equiv x_{i}-x_{i+1}$ e $\tau$ é o conjunto dos índices $\left\{1_{1}, \cdots i_{N}\right\}$. Lembrando que 6.49 é uma distribuição e que para uma distribuição $\phi(x+a)(f(x))=\phi(x)(f(x-a))$. Na verdade, toda simetria de uma distribuição se expressa como:

$$
\phi(x)\left(T_{g} f(x)\right)=\phi(x)(f(x))
$$

onde $T_{g}$ é um elemento da representação do grupo no espaço de funções teste, não necessariamente da forma $T_{g} f(x)=f\left(T_{g}^{\prime} x\right)$, onde $T_{g}^{\prime}$ é uma representação regular do grupo como difeomorfismos nas coordenadas do espaço-tempo.

A segunda propriedade importante é que a transformada de Fourier de $F_{\tau}$ é uma distribuição com suporte(definição no footnote 19 do capítulo 5 concentrado na região dos pontos $\sigma\left(P^{\mu}\right)$, espectro do operador de quadrimomento:

$$
\operatorname{supp} \mathcal{F}\left[F_{\tau}\right] \in \sigma\left(P^{\mu}\right) .
$$

Nos restringindo a realizações do grupo $\mathcal{G}_{P}$ que realizam a álgebra $\mathfrak{p}_{\mathcal{N}}$, assumindo-se uma generalização da condição espectral, postulado 2 do capítulo 5 , que afirma que o espectro do gerador de translações temporais é positivo definido, $\sigma\left(P^{\mu}\right)$ é o fechamento linear convexo da deformação do cone de luz do futuro $V_{\mathcal{G}_{P}}^{+}$, que denotaremos $\overline{V_{\mathcal{G}_{P c}}^{+}}$, conjunto de todas as combinações lineares do tipo $\lambda p^{\mu}+(1-\lambda) p^{\mu \prime}$, onde $p^{\mu}, p^{\mu \prime} \in V_{\mathcal{G}_{P}}^{+}, \lambda \in[0,1]$ (isso será provado na subseção 8.4.2.

A propriedade 6.51 é essencialmente consequência da identidade:

$$
\int d^{4} a e^{i a p} U^{-1}(a) \Psi=0, \text { se } p \notin \sigma\left(P^{\mu}\right)
$$

onde a é uma translação de $\mathfrak{g}$, identidade obtida colocando-se $\Psi$ na representação de energia-momento $\Psi\left(P^{\mu}, \eta\right)$. E que a transformada de Fourier de $F_{\tau}$ com respeito à variável 
$\xi_{j}$ é proporcional a:

$$
\int d^{4} a e^{i p_{j} a} F_{\tau}\left(\xi_{1}, \cdots, \xi_{j}+a, \cdots, \xi_{N-1}\right)=\left\langle 0\left|\Phi_{i_{1}}\left(x_{1}\right) \cdots \int d^{4} a e^{i p_{j} a} \Phi_{1_{2}}\left(x_{j}-a\right) \cdots \Phi_{i_{N}}\left(x_{N}-a\right)\right| 0\right\rangle,
$$

apesar da definição de transformada de Fourier aplicável à distribuições 5.72 .

A terceira propriedade importante ocorre se assumirmos primeiro que, no espaço de Hilbert onde se realiza $\mathcal{G}_{P}$, existe e é único (a menos da normalização) um estado invariante pela ação do grupo, $|0\rangle$, a transformada de Fourier da seguinte função de dois pontos do campo escalar 6.45 com $V_{i j}=I$ ) e além disso, satisfaz uma condição ligada à existência de partículas estáveis conhecida na teoria de campos ususal como condição de gap de massa: Na teoria relativística, p Operador $P^{2}$ formado pelos geradores da representação que aparece na regra de covariância dos campos 6.43 possui um autovalor discreto $m^{2}$, associado a uma partícula estável de massa $m$ e nenhum autovalor entre 0 e $m^{2}$, isto é, existe uma massa mínima das partículas da teoria. Esta condição se generaliza como: O Operador $C(E, P)$ possui um autovalor discreto $\lambda_{0}$ e nenhum outro autovalor entre 0 e $\lambda_{0}$.

Assumindo isso,

$$
\left\langle 0\left|\Phi^{*}(x) \Phi(y)\right| 0\right\rangle
$$

se escreve como:

$$
\tilde{F}_{2}(p)=a \delta(p)+\theta\left(p^{0}\right) \int_{M_{\mathcal{G}_{P}}} \delta(C(E, p)-\lambda) d \sigma(\lambda),
$$

onde $\sigma(\lambda)$ é uma função não decrescente de crescimento no máximo polinomial, a integral é do tipo Stieltjes (footnote 4 do capítulo anterior) e $\theta\left(p^{0}\right)=1$ se $p^{0} \geq 0$ e 0 em caso contrário. Esta é uma generalização não relativística da representação de Källén-Lehmann.

Isso decorre primeiramente da hipótese de positividade do produto interno do espaço de Hilbert, que se traduz na positividade de Wightman ${ }^{17}$.

$$
\iint d^{4} x d^{4} y\left\langle 0\left|\Phi^{*}(x) \Phi(y)\right| 0\right\rangle \bar{f}(x) f(y) \geq 0,
$$

para toda função teste $f(x)$, que, dado 6.49 e a regra de convolução, se traduz no espaço de Fourier segundo:

$$
\int d^{4} p \tilde{F}_{2}(p) \overline{\tilde{f}}(p) \tilde{f}(p) \geq 0
$$

\footnotetext{
${ }^{17}$ A qual não é válida nas teorias de gauge. Mais geralmente, nem todos os campos podem ser realizados num espaço de Hilbert de métrica positivo-definida satisfazendo 6.45
} 
que implica $\tilde{F}_{2}(p)$ uma distribuição positiva definida e, pelo argumentado na vizinhança de 6.1, é uma medida de integração no espaço de Fourier. Mais ainda, uma medida de integração que satisfaz:

$$
\int d \mu T_{\mathcal{G}_{P}}^{\mathcal{F}}[\tilde{f}(p)]=\int d \mu \tilde{f}(p)
$$

em que $T_{\mathcal{G}_{P}}^{\mathcal{F}}$ é a representação do grupo $\mathcal{G}_{P}$ no espaço de Fourier obtida via $T_{\mathcal{G}_{P}}^{\mathcal{F}}[\tilde{f}]=$ $\mathcal{F}\left[T_{\mathcal{G}_{P}} f\right]$. A parte transacional dessa representação é, assim como no caso relativístico,

$$
T_{a}^{\mathcal{F}}[\tilde{f}(p)]=e^{-i p a} \tilde{f}(p)
$$

Estas geram uma representação unitária de $\mathcal{G}_{P}$ no espaço das funções teste Fourier transformadas, visto que $T_{\mathcal{G}_{P}}$ define, via

$$
T_{\mathcal{G}_{P}}^{f}\left[f\left(x^{\mu}\right)\right]=f\left(T_{\mathcal{G}_{P}}\left(x^{\mu}\right)\right),
$$

uma representação unitária no espaço de Hilbert obtido completando o espaço das funções teste com respeito ao produto interno $(f, g)=\int d^{4} x f^{*}(x) g(x)$, produto com respeito ao qual a transformada de Fourier é uma transformação unitária.

De fato,

$$
\left(T_{\mathcal{G}_{P}}^{f}[f], T_{\mathcal{G}_{P}}^{f}[g]\right)=\int d^{4} x T_{\mathcal{G}_{P}}^{f}\left[f^{*}(x) g(x)\right]=(f, g)
$$

dada a propriedade 4 do grupo. O que também pode ser escrito como:

$$
\int d^{4} x X_{i}\left(f^{*}(x) g(x)\right)=0
$$

onde $X_{i}$ é uma transformação infinitesimal do grupo. Assim sendo, 6.58 se escreve como:

$$
\int d \mu \tilde{X}_{i} \tilde{f}=0
$$

onde $\tilde{X}_{i}$ é um operador autoadjunto gerador da representação de um subgrupo de $\mathcal{G}_{P}$, via Teorema de Stone 3, no espaço das funções teste Fourier transformadas. Mas toda medida de integração se escreve como:

$$
\int d \mu \tilde{f}=\lim _{N \rightarrow \infty} \int d^{4} p g_{N}(p) \tilde{f}
$$

$g_{N}(p)$ uma função de crescimento no máximo polinomial, de modo que:

$$
\int d^{4} p g_{N}(p) \tilde{X}_{i} \tilde{f}=\int d^{4} p\left[g_{N}(p), \tilde{X}_{i}\right] \tilde{f}=0
$$


que decorre de

$$
\int d^{4} p T_{\mathcal{G}_{P}}^{\mathcal{F}} \tilde{f}=\int d^{4} p \frac{1}{(2 \pi)^{2}} \int d^{4} x e^{i p x} f\left(T_{\mathcal{G}_{P}}(x)\right)=\int d^{4} x \delta(x) f\left(T_{\mathcal{G}_{P}}(x)\right)=\int d^{4} p \tilde{f},
$$

em que utilizamos a propriedade 3 do grupo, implicando em:

$$
\int d^{4} p \tilde{X}_{i} \tilde{f}=0
$$

para todo $\tilde{f}$. Mas por 6.59 os geradores das translações geram a álgebra das funções contínuas de $p^{\mu}$, as coordenadas do espaço de fases, assim sendo: $\left[g_{N}(p), \tilde{X}_{i}\right]=\zeta_{N}(p)$, $\zeta_{N}(p)$ um função algébrica de $p^{\mu}$, dado a propriedade 6, donde 6.65 para todo $\tilde{f}$ implica $\left[g_{N}(p), \tilde{X}_{i}\right]=0$.

Ou seja, $g_{N}(p)$ é um Casimir obtido como função dos geradores das funções no espaço de fases, os quais são os geradores das translações no espaço-tempo 6.59. Mas, por hipótese, só existem dois com estas propriedades, $C(E, P)$ e $\theta(E)$, melhor dizendo, só existem dois algebricamente independentes. Assim sendo, a álgebra gerada por $C(E, P)$ e $\theta(E)$, os polinômios positivo-definidos de $C(E, P)$ e $\theta(E), F_{N}(C(E, P), \theta(E))$, geram as medidas invariantes segundo:

$$
\int d \mu \tilde{f}=\lim _{N \rightarrow \infty} \int d^{4} p \theta(E) F_{N}(C(E, P), \theta(E)) \tilde{f}
$$

Mas $\theta^{n}(E)=\theta(E)$, a menos que $n=0$, de modo que reescrevemos os polinômios $F_{N}$ segundo

$$
F_{N}=F_{N}^{(1)}(C(E, P))+\theta(E) F_{N}^{(2)}(C(E, P))
$$

A relação entre o momento da teoria e o suporte da medida $d \mu, 6.51$, mais a condição espectral de que a energia é positivo-definida, juntamente com conjunto de autovalores de $C(E, P)$ que o satisfaz, segundo 7, implica que apenas as funções $F_{N}^{(1,2)}(z)$ com suporte em $M_{\mathcal{G}_{P}}$ são admissíveis. Mas essa restrição não é suficiente para garantir $E \geq 0$, precisamos restringir a funções da forma $g_{N}(p)=\theta(E) F_{N}^{(2)}(C(E, P))$. Mas podemos escrever todas essas funções $F_{N}^{(2)}(z)$ da forma:

$$
F_{N}^{(2)}(z)=\int_{\lambda \in M_{\mathfrak{g}}} \delta(z-\lambda) F_{N}^{(2)}(\lambda)
$$

$\rho(\lambda) \geq 0$. Mas não estamos interessados apenas nas funções, mas também nas distribuições positivo-definidas em $z$, as quais se escrevem como (veja 8.4.1):

$$
\int_{\lambda \in M_{\mathfrak{g}}} \delta(z-\lambda) d \sigma(\lambda),
$$


para uma função monotonamente crescente $\sigma(\lambda)$.

Mas porque existe um vácuo invariante, o suporte de $d \mu$ contém $p^{\mu}=0$ e este é um ponto isolado pela condição de gap de massa. De modo que $\int d \mu \tilde{f}=\phi_{1}(\tilde{f})+\phi_{2}(\tilde{f})$ onde $\phi_{1}$ é uma distribuição com suporte em $p^{\mu}=0$ e $\phi_{2}$ com suporte no conjunto dos $C(E, P) \in M_{\mathcal{G}_{P}}$, mas a única distribuição positiva definida com suporte contido num ponto é a delta de Dirac, a menos de uma constante multiplicativa, que justifica o termo $a \delta(p)$.

Podemos reformular 6.55, considerando que:

$$
\int \theta(E) \delta(C(E, P)-\lambda) f(E) d E=\frac{1}{2 \pi i} \oint d E \frac{f(E)}{C(E, P)-\lambda},
$$

para toda função teste analítica $f(E)^{18}$, onde na integral à direita, a integral em $p^{0}$ é realizada no plano complexo num contorno que inclui os polos $C(E, P)=\lambda, E \geq 0$ e exclui aqueles em que $E<0,19$

Assim sendo, 6.55 se reescreve como:

$$
\tilde{F}_{2}(p)=C \delta(p)+\frac{1}{(2 \pi)^{4}} \int_{M_{\mathfrak{g}}} \frac{i d \sigma(\lambda)}{C(E, p)-\lambda}
$$

Para finalizar esse capítulo, utilizaremos a estrutura da função de dois pontos 6.72 para analisar a estrutura de simetria que emerge da prescrição 6.34. Para exemplificar, utilizaremos a não comutatividade canônica $\left[x^{\mu}, x^{\nu}\right]=i \Theta^{\mu \nu}, \Theta^{\mu \nu}$ constante. Para evitar entrar em questões como integral de trajetória e renormalização, citaremos apenas o resultado do cálculo perturbativo da função de dois pontos (ordenada no tempo) quando o termo de interação $\frac{g \phi^{4}}{4 !}$ considerando contribuição de alguns dos diagramas de Feymann, os diagramas de loop:

$$
G(p)=\mathcal{F}\left[F(\xi)=\left\langle 0\left|T \phi\left(x_{1}\right) \phi\left(x_{2}\right)\right| 0\right\rangle\right]=\frac{i}{p^{2}-\frac{g^{2}}{256 \pi^{2}} \ln \frac{1}{M^{2} p \circ p}+M^{2}},
$$

onde $p \circ k=p_{\mu} \Theta_{\lambda}^{\mu} \Theta^{\lambda \nu} k_{\nu}$, enquanto que a função de dois pontos da teoria livre nos dá, devido a 6.37, a relação de dispersão usual.

Essa é uma característica no mínimo estranha, ou mesmo indesejável. Ao definir a teoria pela prescrição 6.34, não existe uma estrutura de simetria aplicável a todas as teorias neste

\footnotetext{
${ }^{18}$ As funções teste analíticas formam um conjunto denso no espaço de funções teste.

19 Em alguns casos como o relativístico, esta prescrição é equivalente a deslocar o polo de $\frac{1}{C(E, P)-\lambda}$ segundo $\frac{1}{C(E, P)-\lambda+i \epsilon}$
} 
espaço. Cada uma, descrita por sua particular lagrangiana, segue uma simetria diferente. Consideraremos no capítulo seguinte uma prescrição proposta por essa tese que supera essa dificuldade. 


\section{A Inflação Algébrica I:}

\section{A estrutura algébrica admissível e uma primeira formulação para a física inflacionária não relativística}

\subsection{Introdução}

O modelo de inflação discutido nos capítulos 3 e 4 é apontado na literatura por alguns como insatisfatória e atormentada por problemas conceituais Brandenberger (2008) e Brandenberger (1999). Alguns dizem que um modelo melhor deveria prever não apenas o padrão de perturbações cosmológicas, mas a amplitude. Outros alegam que o mecanismo de inflação não sobreviveria à tentativa de imersão em modelos de física Trans-Planckiana. Mas ao assumirmos a descrição da matéria local e relativística, principalmente o requerimento de renormalizabilidade Brandenberger (1998), não restam alternativas além de introduzir campos escalares com potenciais de uma forma específica. Para considerar uma classe maior de modelos, talvez com melhores características, precisamos relaxar as hipóteses sobre as quais repousa o modelo padrão da inflação. Pode-se argumentar que enquanto não em desacordo com as observações, não há problemas com o modelo, mas estudar a inflação submetida a modificações dos postulados que levam à física padrão pode não apenas levar a um mecanismo suficientemente robusto para sobreviver ao mergulho na física de altas energias, como leva a um mecanismo para testar a compatibilidade das observáveis cosmológicas com modificações das premissas fundamentais da física.

Para contemplar a sensibilidade do mecanismo inflacionário a modificações das premissas fundamentais, uma violação da localidade em altas energias pode, a princípio, fornecer 
uma alternativa ao problema do horizonte, permitindo vínculos entre pontos além do horizonte. Já contemplado na literatura é o efeito da velocidade da luz variável Albrecht e Magueijo (1999), que pode ser modelado como uma violação da simetria Local de Lorentz, a qual alarga o cone de luz em altas energias, conectando pontos até então desconectados. Na implementação original, a velocidade da luz é um campo que se acopla a todos os demais da mesma maneira que a constante $c$ o faz, e dada uma dinâmica particular, tem-se o mesmo efeito da inflação, com uma possível solução ao problema da constante cosmológica (não como problema de renormalização de energia de vácuo, veja comentário em Albrecht e Magueijo (1999)) 1.

Ainda no cenário de deformação das simetrias locais, temos a inflação não comutativa Alexander e Magueijo (2001), Alexander et al. (2003) um mecanismo para a inflação que tem o potencial de fazer da inflação consequência natural das leis físicas derradeiras. No capítulo anterior, discutimos como a álgebra é uma linguagem alternativa para a física. Aqui demonstraremos que a inflação não comutativa pode ser o caminho de uma linguagem algébrica para a inflação. Além disso, um robusto mecanismo de inflação que sobreviveria até mesmo se a física derradeira admitisse uma estrutura de simetrias mais geral que aquela empregada na física padrão, a qual se sugere ser suficientemente poderosa para codificar as simetrias de um espaço não comutativo.

Começamos a descrever os resultados originais desta tese, que coloca o problema da seguinte maneira: assume uma estrutura algébrica suficientemente poderosa para codificar o que se poderia entender por simetria, tanto do espaço-tempo quântico, quanto clássico, e então, através das ferramentas da álgebra, estuda como mergulhar, de maneira consistente, essa estrutura na teoria quântica relativística da matéria e a possibilidade deste esquema recuperar as equações fenomenológicas do referido modelo.

\subsection{A abordagem da teoria de grupos para a inflação}

A ideia básica explorada em Alexander et al. (2003) é que o efeito (na cosmologia) de modelos genéricos de espaço-tempo não comutativo pode ser codificado no seu caráter não relativístico (discutido em 6.4) sendo este efeito modelado numa relação de dispersão

\footnotetext{
${ }^{1}$ Não como mecanismo de regularização da energia do vácuo, mas como um mecanismo para ajustar o valor da constante cosmológica de Einstein a um valor arbitrariamente pequeno
} 
modificada que afetaria o cálculo da função de partição canônica da radiação, que por sua vez afeta o universo primordial em equilíbrio termodinâmico local.

Que a física de um espaço-tempo quântico, ou melhor, sua fenomenologia cosmológica, poderia ser inteiramente vertida na relação de dispersão e que o cálculo da função de partição canônica não sofreria modificações adicionais, que poderiam até invalidar o mecanismo proposto, não era um fato estabelecido na literatura, mas uma hipótese, que os próprios autores argumentavam necessitar de uma averiguação. Este foi um dos principais objetivos dessa tese: formalizar ou sugerir uma modificação na inflação não comutativa e colocá-la como um mecanismo robusto de inflação, não apenas como uma possível consequência de um espaço-tempo não comutativo, mas um mecanismo genérico de inflação não relativística.

De fato, consideraremos um universo primordial cuja principal componente é a radiação, não um campo escalar. Enquanto a gravidade é tratada classicamente, toda a influência da componente material na evolução da geometria do universo se dá pelo tensor energia-momento. No equilíbrio termodinâmico, desde que a física que governa a radiação preserve localmente a invariância translacional (espacial e temporal) e a invariância rotacional, o tensor energia-momento resultante (no referencial localmente inercial) herdará estas simetrias, e, pelo argumento em 3.24, será o tensor de um fluido perfeito 3.23 , que se traduz no espaço-tempo curvo como 3.24 . A porção do universo em equilíbrio local é a união de um conjunto de sistemas idênticos (constituído do conteúdo material típico da região em equilíbrio) postos a interagir e, se assumirmos a extensividade da energia, constitui um ensemble canônico. Seu comportamento é, por tanto, descrito pela função de partição canônica, a partir da qual é possível calcular a densidade de energia e pressão que descrevem completamente o fluido. Poderíamos, a princípio, seguir o paradigma usual da física, em que toda a sua formulação decorre de uma ação estacionária e de um esquema de quantização, e calcular a função de partição canônica por essa abordagem. Isso nos coloca num problema de determinação da ação que codifica nossas hipóteses sobre a física de altas energias, tal como 6.34. Alternativamente, seguiremos a prescrição de Wigner, em que o problema fundamental da física quântica invariante pela representação de um grupo é a realização do mesmo segundo a noção quântica de simetria 5.16 , que nos coloca no problema de determinar a estrutura algébrica do grupo $\mathfrak{g}$, informação que o define, 
que melhor codifica nossas hipóteses sobre a física de altas energias, de tal modo que a função de partição canônica seja determinada pela representação que descreve a matéria. Nesse sentido, a discussão do capítulo 5 sugere a relevância da relação de dispersão, tal como sugere Alexander et al. (2003), e que se estenderia ao grupo $\mathfrak{g}$ desde que possamos assegurar certas similaridades entre $\mathfrak{g}$ o grupo de Poincaré.

Com efeito, como argumentado no capítulo 5, de acordo com o teorema de Wigner 2 , uma simetria quântica, a qual é definida por 5.16 , pode ser estendida de raios, descrevendo os estados, para o espaço de Hilbert como transformações lineares unitárias ou antilineares antiunitárias. Para o subgrupo conexo $P_{+}^{\uparrow}$ (para o qual o subgrupo de Lorentz satisfaz $\Lambda_{0}^{0}>0$ e $\operatorname{det} \Lambda=1$ ), o qual exclui reflexões espaciais e reversões temporais, o problema se reduz a construir representações unitárias (como argumentado na vizinhança de 5.43. Este problema, por sua vez, resume-se a construir as representações irredutíveis em termos das quais quaisquer outras podem ser construídas (4). As representações irredutíveis são identificadas como compondo o espaço de Hilbert de uma partícula isolada (5.64). A relação de dispersão é uma informação essencial neste esquema, porque é um função autoadjunta dos geradores das translações espaciais e temporais (uma subálgebra comutativa), que comuta com todos os outros geradores do grupo de simetria (Casimir da álgebra de Lie) e define um operador limitado $\left(e^{i C(p)}\right)$ que comuta com todo elemento do grupo. Por uma versão em dimensão infinita do lema de Shur, Teorema 1, uma representação unitária (ou mais geralmente autoadjunta) de um grupo é irredutível se e somente se todo operador limitado que comuta com qualquer elemento do grupo é multiplo da identidade.

Existe um número infinito de representações que podem ser construídas pelo conhecimento das representações irredutíveis. Numa primeira aproximação, a radiação do universo primordial pode ser descrita por um conjunto de fótons livres, sendo a interação o mecanismo que particiona a energia e estabelece o equilíbrio, mas não contribui apreciavelmente para a energia total. A situação é análoga à Hamiltoniana:

$$
H\left(q_{i}, p_{j}\right)=\sum_{i} \frac{p_{i}^{2}}{2 m}+\sum_{i j} V\left(q_{i}-q_{j}\right),
$$

no qual a hipótese ergótica diz que toda a região acessível do espaço de fase, a qual é definida pelo vínculo de conservação da energia, é acessada com a mesma probabilidade ao longo da evolução do sistema. Esta região é na verdade uma complexa variedade no espaço de fases, mas no limite em que a separação média entre as partículas seja tal que o 
termo de interação tenda a zero comparada a energia cinética, a mecânica estatística deste sistema é determinada pelo termo $\sum_{i} \frac{p_{i}^{2}}{2 m}$ da teoria livre, delimitando a região $\sum_{i} \frac{p_{i}^{2}}{2 m}=E$ e assegurando a extensividade da energia.

Consideremos, então, a representação que descreve essa situação, e calculemos a função de partição canônica $Z(\beta, V), \beta=\frac{1}{k_{B} T}$ e $V$ o volume compreendido pela radiação, de modo que este cálculo dependa apenas de hipóteses comuns entre o grupo de Poincaré e um grupo alternativo g. Na subseção 5.2.3, introduzimos o conceito de soma direta de representações como uma maneira de construir uma representação inequivalente a partir de duas outras conhecidas, introduziremos agora o conceito de produto direto ou tensorial:

Dados espaços de Hilbert $\mathcal{H}_{1}$ e $\mathcal{H}_{2}$ podemos definir um terceiro, $\mathcal{H}_{3}=\mathcal{H}_{1} \otimes \mathcal{H}_{2}$, cuja base é constituída dos pares ordenados $\Psi_{i} \otimes \Phi_{j}, \Psi_{i}, \Phi_{j}, i, j=1,2, \cdots$ as bases dos espaços $\mathcal{H}_{1}$ e $\mathcal{H}_{2}$ respectivamente, e o produto $\left(\Psi_{i} \otimes \Phi_{j}, \Psi_{k} \otimes \Phi_{l}\right)_{\otimes}=\left(\Psi_{i}, \Psi_{k}\right)_{1} \cdot\left(\Phi_{j}, \Phi_{l}\right)_{2}$, sendo $(\cdot, \cdot)_{1,2}$ o produto interno de $\mathcal{H}_{1}$ e $\mathcal{H}_{2}$ respectivamente. Dado um operador $U_{1}$ no espaço de Hilbert $\mathcal{H}_{1}$ e $U_{2}$ em $\mathcal{H}_{2}$, definimos o operador $U_{1} \otimes U_{2}$ em $\mathcal{H}_{1} \otimes \mathcal{H}_{2}$ segundo

$$
U_{1} \otimes U_{2}\left(\sum_{i j} c_{i j} \Psi_{i} \otimes \Phi_{j}\right)=\sum_{i j} c_{i j}\left(U_{1} \Psi_{i}\right) \otimes\left(U_{2} \Phi_{j}\right)
$$

Analogamente, dada duas representações do grupo $\mathfrak{g}$ em $\mathcal{H}_{1}$ e $\mathcal{H}_{2}, \mathcal{U}_{1}(\mathfrak{g})$ e $\mathcal{U}_{2}(\mathfrak{g}), \mathcal{U}_{1}(\mathfrak{g}) \otimes$ $\mathcal{U}_{1}(\mathfrak{g})$ define uma representação em $\mathcal{H}_{1} \otimes \mathcal{H}_{2}$. Sendo $\mathcal{U}_{1}(\mathfrak{g})$ e $\mathcal{U}_{2}(\mathfrak{g})$ representações unitárias regulares, fortemente contínuas segundo definição no Teorema $3,7.2$ define uma representação unitária regular, que por sua vez define uma representação da álgebra de Lie:

$$
X_{i}^{\otimes}=X_{i}^{(1)} \otimes I+I \otimes X_{i}^{(2)}
$$

onde $X_{i}^{(j)}$ os geradores da álgebra de Lie das representações $\mathcal{U}_{1}(\mathfrak{g})$ e $\mathcal{U}_{2}(\mathfrak{g})$ respectivamente.

Sendo $\mathcal{U}_{\lambda}(\mathcal{P})$ um representação irredutível do grupo de Poincaré ( $\lambda$ denota um conjunto de índices que rotulam as representações irredutíveis, os possíveis autovalores dos operadores de Casimir 5.53), atuando no espaço de Hilbert de uma partícula $\mathcal{H}_{\lambda}$, a representação $\mathcal{U}_{\lambda}^{\otimes N}(\mathcal{P})$, o produto de $N$ representações idênticas, que atua no espaço de Hilbert $\mathcal{H}_{\lambda}^{\otimes N}$, descreve um conjunto de partículas não interagentes, visto que a evolução dos estados no tempo ocorre de maneira independente para cada partícula. Ou melhor, descreve um conjunto de partículas inequivalentes. Num espaço de Hilbert de partículas idênticas, todos os estados são invariantes por permutações de duas partículas quaisquer 
$P$, ou seja, $P \Psi=e^{i \theta} \Psi$ e como $P^{2}=1$, $e^{i \theta}= \pm 1$. Logo, para construir o espaço de Hilbert de partículas idênticas introduzimos as operações de simetrização:

$$
S_{N}\left(\psi_{1} \otimes \cdots \otimes \psi_{N}\right)=\frac{1}{N !} \sum_{\sigma} \psi_{\sigma(1)} \otimes \cdots \otimes \psi_{\sigma(N)},
$$

onde $\sigma$ é uma permutação dos índices, e a antissimetrização:

$$
A_{N}\left(\psi_{1} \otimes \cdots \otimes \psi_{N}\right)=\frac{1}{N !} \sum_{\sigma} \operatorname{sign}(\sigma) \psi_{\sigma(1)} \otimes \cdots \otimes \psi_{\sigma(N)},
$$

onde $\operatorname{sign}(\sigma)$ é 1 se $\sigma$ envolve um número par de permutações entre dois elementos e -1 em caso contrário, que definem os espaços:

$$
\mathcal{H}_{S}^{N}=\left\{\psi \in \mathcal{H}^{N} ; S_{N} \psi=\psi\right\}
$$

e

$$
\mathcal{H}_{A}^{N}=\left\{\psi \in \mathcal{H}^{N} ; A_{N} \psi=\psi\right\}
$$

Denotemos o produto tensorial das representações $\mathcal{U}_{\lambda}(\mathcal{P})$ restrita aos subespaços $\mathcal{H}_{S, A}^{N}$ como $\left(\mathcal{U}_{\lambda}^{\otimes N}\right)_{S, A}(\mathcal{P})$. Do fato experimental de que o número de fótons não se conserva, o conteúdo de energia de uma dada região dependerá do número (variável) de partículas dessa região. O espaço de Hilbert que descreve os estados da matéria numa região do ensemble canônico é:

$$
U=\sum_{N=0}^{\infty} \oplus\left(\mathcal{U}_{\lambda}^{\otimes N}\right)_{S}(\mathcal{P})
$$

que é definida em:

$$
\mathcal{H}=\sum_{N=0}^{\infty} \oplus\left(\mathcal{H}_{\lambda}^{\otimes N}\right)_{S}
$$

( $N=0$ corresponde a representação trivial do vácuo). Estudamos a realização das representações irredutíveis no capítulo 5

Para calcular a função de partição canônica, $Z(\beta, V)=\operatorname{Tr}\left(e^{-\beta H}\right)$, precisamos da Hamiltoniana associada à representação 7.8 .

$$
H_{F}=\sum_{N=1}^{\infty} \oplus(H \otimes I \cdots \otimes I+I \otimes H \otimes \cdots \otimes I+\cdots+I \otimes I \cdots \otimes H)_{N},
$$

mas sendo $\mathcal{U}_{\lambda}(\mathcal{P})$ uma representação irredutível, o lema de Shur 1 diz que $H$ e $P^{i}$ são relacionados via $C\left(H, P^{i}\right)=m^{2}$, que no caso relativístico, determina unicamente $H$ em função de $P^{i}$ :

$$
H=\sqrt{m^{2}-\vec{P}^{2}}
$$


Assim sendo, se pudermos determinar o espectro de $P^{i}$ e a dimensão do autoespaço associado a cada autovalor de $P^{i}$, o suficiente para representá-lo no espaço de Hilbert, $H$ estará determinado. Mas para determinar a dependência da função de partição com o volume, precisamos confinar a radiação neste volume, que pode ser escolhido como uma caixa cúbica. Essa dependência é que determina a pressão do fluido e decorre de um vínculo que o confinamento implica na representação da translação espacial no espaço de Hilbert. De fato, suponha que a radiação esteja confinada na região do espaço entre $0 \leq x, y, z \leq L$, podemos impor $U_{i}(0)=U_{i}(L)$, onde $U_{i}$ é a realização da translação espacial na i-ésima direção de $\mathcal{U}_{\lambda}(\mathcal{P})$. Na verdade, a exata condição de contorno dentro da caixa não é fisicamente observável, tal informação necessita do conhecimento preciso da informação física em pontos isolados, mas experimentos físicos podem apenas nos dar informação sobre vizinhanças em torno de um ponto particular. Assim sendo, 5.47 implica na quantização do momento:

$$
p_{j}=\frac{2 \pi \hbar n_{j}}{L}, n_{j} \text { um inteiro. }
$$

Podemos denotar os estados $\mathcal{H}_{S}^{N}$ de um modo alternativo:

$$
\left|n_{1}, n_{2}, \cdots\right\rangle=S\left(\psi_{1}^{\otimes n_{1}} \otimes \psi_{2}^{\otimes n_{2}} \otimes \cdots\right),
$$

onde $\psi_{i}$ são os elementos de uma base dos estados de uma partícula, escolhidos como a base contável de autoestados do momento de uma partícula, que poderiam ser rotuladas pelos inteiros $\left(n_{x}, n_{y}, n_{z}\right)$. De acordo com 5.77, numa representação irredutível, a dimensão do autoespaço associado a qualquer conjunto admissível de autovalores de $\left(H, P^{i}\right)$ é a mesma, o que combinado com 7.11 implica que a dimensionalidade do autoespaço associado a qualquer conjunto de autovalores de $P^{i}$ do momento é a mesmo, denotada $g$. Numa teoria não invariante por paridade e reversão temporal, $g$ é igual à dimensionalidade da representação do pequeno grupo 5.85, que deixa o quadrimomento invariante, que é 1 para para todas as representações tais que $m^{2}=0$, associado aos possíveis valores $\sigma$ de helicidade, projeção do momento angular na direção do momento, mas ao assumirmos que esta representação descreve uma teoria invariante por paridade, que reverte o sinal o momento e por consequência conecta helicidades opostas $\pm \sigma$, segundo o argumentado em 5.88 $g=2$, o que equivale a estender o pequeno grupo incluindo transformações $U(R) P$, onde $R$ é a rotação de $180^{\circ}$ em torno de um eixo ortogonal ao momento e $P$ a paridade 
$\vec{p} \rightarrow-\vec{p}$. Assim sendo:

$$
\operatorname{Tr}\left(e^{-\beta H}\right)=\sum_{n_{j}}\left\langle n_{1}, n_{2}, \cdots\left|e^{-\beta H_{F}}\right| n_{1}, n_{2}, \cdots\right\rangle=\prod_{i}\left(\sum_{n_{i}} e^{-\beta n_{i} E_{i}}\right)^{g}=\prod_{i}\left(\frac{1}{1-e^{-\beta E_{i}}}\right)^{g}
$$

De modo que:

$$
\rho(\beta, V)=-\frac{1}{V} \frac{\partial}{\partial \beta} \ln (Z(\beta, V))=\frac{1}{V} \sum_{i} \frac{g E_{i}}{e^{\beta E_{i}}-1}
$$

$\mathrm{e}$

$$
p(\beta, V)=\frac{1}{\beta} \frac{\partial}{\partial V} \ln (Z(\beta, V))=-\sum_{i} \frac{g}{e^{\beta E_{i}}-1} \frac{\partial E}{\partial p^{i}} \frac{\partial p^{i}}{d V},
$$

Uma vez explicitado o caminho que conecta o grupo de simetrias local da física com o tensor energia-momento que governa a cosmologia, vemos aqui um potencial mecanismo de aceleração de um universo em expansão adiabática modificando-se a relação de dispersão associada ao grupo. Se esta for escolhida de tal modo que a energia de uma partícula aumente com a diminuição do momento para pelo menos uma parte dos estados acessíveis de partícula, enquanto pudermos assegurar que, com a expansão do universo, o momento diminua, ou seja, $\frac{\partial p^{i}}{d V}<0$, a pressão do fluido pode vir a ser negativa para alguma temperatura. Esta é a ideia da inflação não comutativa. Temos aqui uma importante mudança de paradigma na inflação: Desde sua concepção, o mecanismo inflacionário passava necessariamente por uma fase primordial do universo dominada por campos escalares com um tipo particular de potencial, como discutido em 3, sendo necessário assegurar o decaimento da energia potencial dos campos na matéria ordinária ao final da inflação. Temos aqui um mecanismo que prescinde de campos escalares e de reaquecimento.

É importante ressaltar que a conexão entre inflação e a representação de grupos no espaço de Hilbert nãa foi o ponto de vista explorado no trabalho original da inflação não comutativa Alexander et al. (2003). Essa tese, até então, explora uma mudança de perspectiva que lhe é original. Nesse sentido, a inflação não comutativa poderia ser chamada apenas de inflação não relativística, mas diante de desenvolvimentos da física de altas energias que sugeriam que a ideia de espaço não comutativo pode vir a ser uma característica da física de altas energias, e diante do caráter não relativístico intrínseco ao conceito, discutido em 6.4, este poderia ser um mecanismo conectando a inflação a várias frentes de abordagem à física trans-planckiana, podendo até fornecer um mecanismo inflacionário até então elusivo para a teoria de cordas, ou diferente do que ocorre com a 
inflação de rolamento lento, um mecanismo capaz de sobreviver a correções perturbativas de altas energias. Nesse sentido, os autores conjecturaram que a não comutatividade seria efetivamente modelada, ao menos no que diz respeito à termodinâmica, na mudança da relação de dispersão. Uma conjectura que merece uma averiguação.

Para exemplificar tal mecanismo, os autores postularam uma relação de dispersão da forma:

$$
E^{2}=p^{2} f^{2}(E)
$$

Assumindo que a distribuição de momentos permitidos para a radiação confinada numa caixa de volume $V, 7.12$, bem como a número de graus de liberdade interno dos fótons, permanecem inalterados, decorre que $\Omega(E) d E=\Omega(p) d p$, onde $\Omega(E) d E$ é o número de microestados no intervalo de energia $d E$, enquanto $\Omega(p) d p$ é o número no intervalo de momento $d p$. Reescrevendo $\sum_{i} \rightarrow \int \Omega(E) d E$ em 7.15 e 7.16, temos que a radiação não comutativa é descrita por um fluido perfeito governado pelas equações:

$$
\begin{gathered}
\rho(E, T)=\frac{1}{\pi^{2}} \frac{E^{3}}{\exp E / T-1} \frac{1}{f^{3}}\left|1-\frac{E f^{\prime}}{f}\right| \\
p=\frac{1}{3} \int \frac{\rho(E, T)}{1-\frac{E f^{\prime}}{f}} d E \\
\rho=\int \rho(E, T) d E
\end{gathered}
$$

Aqui tomamos $c=k_{B}=\hbar=1$. Para encontrar um exemplo de comportamento inflacionário, Alexander et al. (2003) utilizou o ansatz:

$$
f=1+(\lambda E)^{\alpha}
$$

onde $\lambda$ é um parâmetro que define a escala de energia da inflação e uma análise numérica encontrou um intervalo de valores de $\alpha$ que permite $\rho+3 p<0$, a condição para aceleração com $\Lambda=0$ segundo 3.29 .

Diante da conexão entre a inflação e a representação de grupos e estruturas algébricas, perspectiva desta tese, os resultados publicados desta são relacionados às questões: Existe de fato um grupo cuja representação no espaço de Hilbert permite replicar o raciocínio que leva à inflação não comutativa, incluindo possiveis escolhas de relação de dispersão inflacionárias? Existe algum restrição física para alguma representação desse grupo? Ele é único? Que condições sobre a sua estrutura algébrica são necessárias e/ou suficientes para 
garantir a validade das equações? Como se modificaria a física associada à quantização de cada possível Lagrangiana envolvendo campos relativísticos? Dada a alta densidade de energia na qual a inflação ocorreria, como assegurar que correções perturbativas oriundas da interação entre os fótons não invalidariam o mecanismo? A ideia de inflação não comutativa é realmente compatível com a ideia de espaço não comutativo? Ela poderia ser codificada na estrutura algébrica das simetrias e de um modo a validar a conjectura dos idealizadores do modelo de que a não comutatividade se traduz na mudança fenomenológica da relação de dispersão de modo a realizar 17.14, ou este cálculo necessitaria de uma revisão o que poderia invalidar o mecanismo ou no mínimo levar à necessidade de diferentes escolhas de relação de dispersão inflacionárias, isto é, se presente a não comutatividade, este mecanismo poderia operar a inflação neste espaço?

\subsection{Estrutura algébrica admissível}

Uma vez que estrutura algébrica encerra em si toda a informação sobre o grupo, a primeira questão tratada é sobre a estrutura algébrica necessária e/ou suficiente para assegurar a validade do cálculo da função de partição da radiação. Em outras palavras, que propriedades tem o grupo de simetria $\mathcal{P}_{\mathcal{N}}$ que realiza a inflação? Após o que, fará sentido perguntar se existe um grupo que realiza a inflação segundo o mecanismo exposto. Para tanto, façamos referência às propriedades do grupo $\mathcal{G}_{P}$ que permitiram generalizar a representação de Källén-Lehmann 6.55 na seção 6.4. A contemplação dessa estrutura é original desta tese

Ao invés de definirmos esse grupo por equações algébricas não triviais envolvendo o produto de seus elementos, definiremos a partir de uma particular representação que chamaremos de representação definidora. Uma vez que este grupo se postula substituir o grupo de Poincaré, o qual é definido por sua ação como transformações no espaço-tempo, $\mathcal{P}_{\mathcal{N}}$ é definido a partir de sua ação no espaço-tempo. Esta prescrição pode ser generalizada para: $\mathcal{P}_{\mathcal{N}}$ é definido por uma representação na $C^{*}$-álgebra do espaço-tempo, $\mathcal{A}_{S T}$, $\mathcal{P}_{\mathcal{N}}: \mathcal{A}_{S T} \rightarrow \mathcal{A}_{S T}$. Esse é essencialmente o conteúdo da propriedade 2 de $\mathcal{G}_{P}$. Sobre a representação definidora, requeremos primeiramente condições que assegurem uma física consistente, tal como a existência de uma representação no espaço de Hilbert segundo o critério de Wigner para simetrias, que pode ser assumida não projetiva. Isso era assegu- 
rado pela propriedade 4 de $\mathcal{G}_{P}$, que tornava a própria representação definidora unitária, mas podemos generalizar essa propriedade pela existência de um estado (funcional linear positivo-definido) $\int: \mathcal{A}_{S T} \rightarrow \mathbb{C}$ no qual a ação do grupo seja unitária $\int(U f)^{*} U g=\int f^{*} g$, $f, g \in \mathcal{A}_{S T}$ e $U \in \mathcal{P}_{\mathcal{N}}$. É necessário que possamos, segundo o critério do teorema de Stone, definir as observáveis da teoria a partir do grupo, observáveis tais como energia e momento. O que é imprescindível, pois a termodinâmica depende de uma Hamiltoniana a ser definida em função da representação do grupo. Dada uma representação unitária no espaço de Hilbert, isso é possível se o grupo for definido a partir de subgrupos de um parâmetro, como na propriedade 1, que se realizam de forma regular $\left(\left\langle\Psi\left|U_{i}\left(\theta_{i}\right)\right| \Psi\right\rangle\right.$ contínuo em $\left.\theta_{i}\right)$, que generaliza a propriedade 2. É necessário também que os geradores dessa representação atuem num domínio comum invariante $\mathcal{D}$, pois as mesmas observáveis são aplicáveis a todos os estados. Como discutido na propriedade 4, esse domínio não necessariamente é o domínio em que os geradores $X_{i}$ são autoadjuntos (domínio esse fundamental na definição de autoadjunto, do contrário, temos outras classes de operadores que não asseguram, por exemplo, autovalores reais), mas essencialmente autoadjuntos, o que no mínimo assegura uma extensão única para operadores autoadjuntos. Em particular, deve existir um subgrupo comutativo $\mathcal{T}$ que identificaremos com as translações espaço-temporais, como requerido na propriedade 2. Isso assegura não apenas que possamos identificar em $\mathcal{P}_{\mathcal{N}}$ a Hamiltoniana e o operador Momento, como assegura que eles comutem. O que assegura que dada qualquer representação dos geradores $X_{i}$, existe uma transformação unitária que coloca os estados na forma:

$$
\Psi=\int d^{3} \mu\left(\Psi_{p^{\mu}}\right) \phi\left(\Psi_{p^{\mu}}\right) \Psi_{p^{\mu}},
$$

em que $d^{3} \mu\left(\Psi_{p^{\mu}}\right)$ é uma medida no espaço de autoestados generalizados linearmente independentes. Não podemos ainda assumir que a dimensão do autoespaço associado ao autovalor $p^{\mu}$ seja independente de $p^{\mu}$.

É necessário que, no domínio invariante $\mathcal{D}$, os geradores formem uma álgebra não trivial, que denotaremos $\mathfrak{p}_{\mathcal{N}}$, propriedade 5. Isto é, $\left[X_{i}, X_{j}\right]=i C_{i j}\left(X_{k}\right)$, onde $C_{i j}\left(X_{k}\right)$ é uma função analítica dos $X_{i}$ não trivialmente satisfeita por qualquer conjunto $X_{i}$. Isso é fundamental, primeiramente para assegurar que um autoestado de energia, um estado estável, num referencial seja também um autoestado de energia (estável), sob transformação de $\mathcal{P}_{\mathcal{N}}$. Para tanto, a álgebra gerada por energia-momento deve ser um ideal, propriedade 6 , 
o que essencialmente assegura

$$
U\left(\mathfrak{g}_{1}\right) P^{\mu} U^{-1}\left(\mathfrak{g}_{1}\right)=G_{\mathfrak{g}}\left(P^{\mu}\right)
$$

em que $\mathfrak{g}_{1} \in \mathcal{P}_{\mathcal{N}}$ e $G_{\mathfrak{g}_{1}}$ é uma realização de $\mathcal{P}_{\mathcal{N}}$ por transformações nas coordenadas de energia-momento (segundo explicação no footnote 15 do capítulo anterior).

A propriedade fundamental é 7, que assegura que podemos construir representações irredutíveis da álgebra $\mathfrak{p}_{\mathcal{N}}$ pelo Lema de Shur 1, representações essas que podem ser escolhidas com energia positiva definida. Dada a propriedade 7.23 , pelo mesmo argumento de 5.77 , asseguramos agora que nas representações irredutíveis, para as quais $\left(p^{0}, p^{i}\right)=(E, \vec{P})$ tal que $C(E, P)=\lambda$ para algum $\lambda$ em $M_{\mathcal{P}_{\mathcal{N}}}$ (definido na propriedade 7), a dimensionalidade do autoespaço de $p^{\mu}$ é independente de $p^{\mu}$ e escrevemos os estados como:

$$
\Psi=\sum_{\sigma} \int d^{3} \mu(p) \phi\left(p^{\mu}, \sigma\right) \Psi_{p^{\mu}, \sigma},
$$

onde $\sigma$ é um índice que pode ser provado discreto no caso de espaço de Hilbert separável.

Requeremos agora que $\mathcal{P}_{\mathcal{N}}$ recupere o grupo de Poincaré no limite de baixa energia e momento. Sejam $C_{k}^{i j(0)}$ as constantes de estrutura da álgebra de Lie de Poincaré 5.495 .51 e sejam $C_{k}^{i j}(E, P)$ funções contínuas tais que $C_{k}^{i j}(0,0)=C_{k}^{i j(0)}$. Escrevamos $\mathfrak{p}_{\mathcal{N}}$ como

$$
\left[X_{i}, X_{j}\right]=i X^{k} C_{k}^{i j}(H, P)
$$

Dado, na representação 7.24 , um estado da forma:

$$
\Psi_{p_{\max }^{\mu}}=\int d \mu(p) \phi(p) \Psi_{p} \quad \operatorname{supp}\{\phi(p)\} \in\left\{E<E_{\max }, P^{j}<p_{\max }^{j}\right\}
$$

a ação dos geradores de $\mathfrak{p}_{\mathcal{N}}$ nesses estados satisfaz:

$$
\left[X^{i}, X^{j}\right] \triangleright \Psi_{p_{\max }^{\mu}}=i X^{k} C_{k}^{i j}(H, P) \triangleright \Psi_{p_{\max }^{\mu}} \rightarrow i X^{k} C_{k}^{i j(0)} \triangleright \Psi_{p_{\max }^{\mu}} \text {, quando } p_{\max }^{\mu} \rightarrow 0
$$

onde supp denota o suporte. Aqui, $C_{k}^{i j}(H, P)$ converge para as constantes de estrutura de Poincaré $\left(C_{k}^{i j(0)}\right)$ quando $E_{\max }$ e $p_{\max }^{i}$ se aproximam de 0 . Aqui, $\triangleright$ detona a ação (como operador) nos estados de uma partícula e $\rightarrow$ denota a convergência forte (isto é, i.e. $\left.\left\|X^{k} C_{k}^{i j}(H, P) \triangleright \Psi-X^{k} C_{k}^{i j 0} \triangleright \Psi\right\| \rightarrow 0\right) 2$. Isto ilustra que os autovalores típicos

\footnotetext{
${ }^{2}$ Para operadores autoadjuntos, ou $\left\|X^{k} C_{k}^{i j}(H, P) \triangleright \Psi-X^{k} C_{k}^{i j}{ }^{0} \triangleright \Psi\right\| \rightarrow 0$, ou não é uma sequ6encia convergente
} 
dos geradores podem efetivamente mudar as relações de comutação. A propriedade 7.25 assegura automaticamente que a álgebra gerada por $H, P$ é um ideal.

Dada a propriedade 7.23 , faz sentido o conceito de pequeno grupo, subgrupo $\mathcal{W}_{p^{\mu}}$ de $\mathcal{P}_{\mathcal{N}}$ na representação 7.24 que deixa um dado quadrimomento $p^{\mu}$ de representação irredutível invariante (5.85). O pequeno grupo de qualquer $p^{\mu}$ de uma mesma representação irredutível é o mesmo (isomórfico), pois numa representação irredutível qualquer $p^{\mu}$ pode ser mandado em qualquer outro pela ação de algum $G_{\mathfrak{g}_{1}}$. Assim sendo, dado $w_{1} \in \mathcal{W}_{p_{1}^{\mu}}$ podemos obter $w_{2} \in \mathcal{W}_{p_{2}^{\mu}}$, dado $G_{\mathfrak{g}_{1}}\left(p_{1}^{\mu}\right)=p_{2}^{\mu}: G_{\mathfrak{g}_{1}} w_{1} G_{\mathfrak{g}_{1}}^{-1}$. Assim sendo, o pequeno grupo que atua em baixa energia e momento deve ser o mesmo que atua em altas energias. Que nos leva ao requerimento de que todo pequeno grupo de $\mathcal{P}_{\mathcal{N}}, \mathcal{W}_{p^{\mu}}$, para $\lambda \in M_{\mathcal{P}_{\mathcal{N}}}$ (definido na propriedade 7) deve ser isomórfico a algum pequeno grupo $\mathcal{W}\left(\Lambda, p^{\mu}\right)$ de Poincaré associado a alguma representação irredutivel de energia positivo-definida. O que assegura também que a dimensão do autoespaço de $p^{\mu}$ é a mesma em certas representações, e o fator $g$ em 7.14 se preserva.

Resta ainda uma última condição: $\mathfrak{p}_{\mathcal{N}}$ deve preservar a álgebra de Lie de $S O(3)$, o grupo de rotações, e $\left[H, M_{i}\right]=0$, onde $M_{i}$ é o gerador do subgrupo de rotação em torno da i-ésima direção. De fato, a descrição termodinâmica de fluido perfeito deixa de valer se o estado termodinâmico deixa de ser invariante pelo grupo de rotações (um fluido perfeito é isotrópico no referencial comóvel ao fluido num dado ponto) e na descrição termodinâmica da matéria, a matéria de cada uma das regiões do universo é descrita por um estado não puro $\omega(A)=\frac{\operatorname{Tr}\left(e^{-\beta H} A\right)}{\operatorname{Tr}\left(e^{-\beta H}\right)}$, onde $A$ é uma observável 3 . O estado é rotacionalmente invariante se $\omega\left(R A R^{-1}\right)=\omega(A)$, onde $R$ é uma rotação. Dada a ciclicidade do traço $\frac{\operatorname{Tr}\left(e^{-\beta H} A\right)}{\operatorname{Tr}\left(e^{-\beta H}\right)}=\frac{\operatorname{Tr}\left(R^{-1} e^{-\beta H} R A\right)}{\operatorname{Tr}\left(e^{-\beta H}\right)}, \forall A \Leftrightarrow\left[M_{i}, H\right]=0$

Observe ainda que se parte das relações de comutação de $\mathfrak{p}_{\mathcal{N}}$ é da forma $\left[X_{i}, X_{j}\right]=$ $C_{i j}^{k} X_{k}, C_{i j}^{k}$ constantes, estas são preservadas em qualquer representação do grupo $\mathcal{P}_{\mathcal{N}}$.

Surge aqui uma sutileza de extrema importância: Para um grupo de Lie, toda representação do grupo induz uma representação da mesma álgebra de Lie. Não necessariamente uma representação $\pi$ do grupo $\mathcal{P}_{\mathcal{N}}$ no espaço de Hilbert $\mathcal{H}_{1}$, a qual é assegurada satisfazer $\pi\left(g_{1}\right) \pi\left(g_{2}\right)=\pi\left(g_{1} g_{2}\right)$, é tal que os geradores representam a mesma álgebra $\mathfrak{p}_{\mathcal{N}}$. Assim

\footnotetext{
${ }^{3}$ Observe a definição de estado como funcional linear positivo definido na álgebra de observáveis dada no capítulo 6, seção 6.2. É possível mostrar que dadas um representação fiel da $C^{*}$-álgebra das observáveis, qualquer estado é da forma $\operatorname{Tr}(\rho A)$, onde $\rho$ é uma matriz de densidade.
} 
sendo, ao deformar a álgebra de Lie de Poincaré, necessitamos considerar dois tipos de representações de $\mathcal{P}_{\mathcal{N}}$, as representações de $\mathcal{P}_{\mathcal{N}}$ que preservam a álgebra $\mathfrak{p}_{\mathcal{N}}, \pi_{\mathcal{A}}$, e as representações do grupo $\mathcal{P}_{\mathcal{N}}$, que não necessariamente preservam $\mathfrak{p}_{\mathcal{N}}$, genericamente denotadas $\pi_{\mathfrak{g}}$. Não necessariamente toda representação $\pi_{\mathfrak{g}}$ é também $\pi_{\mathcal{A}}$. Essa é uma distinção não observada pelos autores originais da inflação não comutativa e que tem implicações profundas que são originais desta tese

\subsection{Sobre a conjectura da tradução da não comutatividade na estrutura algébrica das simetrias locais e a prescrição generalizada para a física não relativística}

\subsubsection{As Hopf álgebras}

Estudemos aqui a hipótese de que podemos versar a ideia de não comutatividade do espaço-tempo na estrutura algébrica de simetrias de modo obter uma estrutura algébrica admissivel.

Foi sugerido na literatura, Agostini et al. (2004), que uma estrutura da álgebra abstrata, chamada de álgebra de Hopf, poderia oferecer uma linguagem natural para codificar a noção de simetria que faria sentido num espaço não comutativo. Em certo sentido, uma simetria que classicamente se manifestaria como o grupo de Poincaré, seria, num contexto quântico, não mapeada na sua representação no espaço de Hilbert, mas quantizada em algum outro sentido. Na ocasião, os autores, ainda partindo do paradigma de que toda a física quântica decorre da quantização de uma lagrangiana, sugeriram, e apenas no caso do campo escalar, uma ação obtida pelo requerimento de invariância com respeito à ação da Hopf álgebra. Este se mostrou um critério alternativo ao descrito em 6.34 para descrever a física do espaço não comutativo. Para obter o principal resultado original dessa tese, partimos dessa sugestão, que uma Hopf álgebra descreve as simetrias da física de altas energias, mas tomamos um caminho diferente, já sugerido pelo que se escolheu discutir nos capítulos anteriores: Definir a teoria da matéria por analogia direta a como Wigner sugere construir a teoria quântica relativística. Isto é, pela representação de grupos (segundo discutido no capítulo 5), mas de algum modo modificado para levar em conta a generalização envolvida no conceito de álgebra de Hopf. A descrição que faremos da teoria 
das Hopf álgebras é baseada em Majid (2000), Podles e Muller (1997) e Aschieri (2007)

Dito de um modo simplificado, a álgebra de Hopf emerge como um conjunto de transformações que atua não somente num espaço vetorial ou topológico, mas numa álgebra, tal como a $C^{*}$-álgebra, e de um modo dependente das operações que definem a álgebra em questão. Como toda a informação que codifica o caráter quântico do espaço se traduz em álgebra, nada mais natural que definir a ação das simetrias como dependentes desta.

Dada a correspondência entre espaços topológicos e álgebras, discutida no capítulo 6 , toda ação de um grupo no espaço, tal como a ação 6.2, induz uma ação na $C^{*}$-álgebra associada, como em 6.3, da qual a ação no espaço pode ser recuperada via o formalismo discutido. Logicamente, não existe noção natural de simetria num espaço topológico. No espaço-tempo físico, esta emerge do requerimento de invariância da velocidade da luz em referenciais inerciais, não resta critério outro para definir as simetrias do espaço-tempo não comutativo que não assumir que, no limite comutativo, esta seja a ação do grupo de Poincaré. Definiremos a álgebra de Hopf de maneira pragmática a partir da maneira como esta atua numa álgebra (onde as operações definidas são o produto associativo e as combinações lineares), que é o caso relevante nesta tese, em vista de um candidato a atuar na $C^{*}$-álgebra.

A álgebra de Hopf $H$ atua numa álgebra $\mathcal{A}$ como um conjunto de transformações não necessariamente inversíveis, $H: \mathcal{A} \rightarrow \mathcal{A}$, sendo esta ação (ou representação) denotada $h \triangleright f, h \in H$ e $f \in \mathcal{A}$. Antes de qualquer coisa, $H$ é uma álgebra, de modo que existe uma multiplicação definida pela composição e combinações lineares a coeficientes reais ou complexos:

$$
\begin{gathered}
\left(h_{1} \cdot h_{2}\right) \triangleright f=h_{1} \triangleright\left(h_{2} \triangleright f\right) \\
\left(\alpha h_{1}+\beta h_{2}\right) \triangleright f=\alpha\left(h_{1} \triangleright f\right)+\beta\left(h_{2} \triangleright f\right) .
\end{gathered}
$$

Especificamos a ação de $H$ no produto de elementos de $\mathcal{A}$ segundo: $h \triangleright(f \cdot g)=\sum_{i}\left(h_{i(1)} \triangleright\right.$ $f) \cdot\left(h_{i(2)} \triangleright g\right)$, ou, utilizando-se de uma notação abreviada:

$$
h \triangleright(f \cdot g)=\left(h_{(1)} \triangleright f\right) \cdot\left(h_{(2)} \triangleright g\right),
$$

onde a regra $\Delta: H \rightarrow H \otimes H$ dada por $\Delta: h \rightarrow \sum_{i} h_{i(1)} \otimes h_{i(2)}$ é chamada de coproduto. Decorre imediatamente que $\Delta$ possui uma propriedade chamada de coassociatividade, $(i d \otimes \Delta) \Delta h=(\Delta \otimes i d) \Delta h$, onde $i d$ é a identidade dentre os operadores $O: H \rightarrow H$, que 
decorre de $H \triangleright(a b c)$, onde aplicamos a regra 7.30 primeiro no par $(a, b c)$, mas que deve produzir o mesmo resultado de aplicarmos primeiro no par $(a b, c)$. Decorre da coassociatividade a generalização de 7.30 para o produto de $N$ elementos:

$$
H \triangleright\left(\prod_{i} f_{i}\right)=\prod_{i}\left(h_{(i)} \triangleright f_{i}\right),
$$

onde a regra $\Delta^{N}: H \rightarrow H^{\otimes N}$ obtida via

$$
\Delta^{N} h=(\Delta \otimes i d \cdots \otimes i d) \cdots(\Delta \otimes i d \otimes i d)(\Delta \otimes i d) \Delta h=h_{(1)} \otimes h_{(2)} \cdots h_{(N)},
$$

produz, pela coassociatividade, o mesmo resultado, ainda que em cada termo $(\Delta \otimes i d \cdots \otimes$ $i d)$ alternássemos a posição do $\Delta$. Nesse sentido, podemos identificar $\Delta \operatorname{com}(\Delta \otimes i d \cdots \otimes i d)$ ao atuar em $H^{\otimes N}$.

Quando um dos elementos na multiplicação é a identidade de $\mathcal{A}$, temos que

$$
\begin{aligned}
& h \triangleright(1 \cdot g)=\left(h_{(1)} \triangleright 1\right) \cdot\left(h_{(2)} \triangleright g\right)=\epsilon\left(h_{(1)}\right) \cdot\left(h_{(2)} \triangleright g\right)=h \triangleright g, \\
& h \triangleright(g \cdot 1)=\left(h_{(1)} \triangleright g\right) \cdot\left(h_{(2)} \triangleright 1\right)=\left(h_{(1)} \triangleright g\right) \cdot \epsilon\left(h_{(2)}\right)=h \triangleright g,
\end{aligned}
$$

onde a regra $\epsilon: H \rightarrow \mathbb{C}$ é chamada de counidade. Se $\Delta: H^{\otimes N} \rightarrow H^{\otimes N+1}$, a counidade faz o caminho inverso e desfaz a aplicação de $\Delta, \epsilon: H^{\otimes N+1} \rightarrow H^{\otimes N}$, onde identificamos $\epsilon$ com $(\epsilon \otimes i d \cdots \otimes i d)$, independente da posição de $\epsilon$. A álgebra $\mathcal{A}$ na qual $H$ atua segundo as regras acima é chamada de $H$-módulo álgebra.

A álgebra de Hopf atua em $\mathcal{A}$ de modo não necessariamente inversível, apesar disso, é definida uma noção generalizada de inversa chamada de antípoda, $S: H \rightarrow H$, que no caso de algum elemento de $H$ ser inversível satisfaz $S(h)=h^{-1}$. A antípoda é (unicamente) definida pelas propriedades $S\left(h_{(1)}\right) \cdot h_{(2)}=h_{(1)} \cdot S\left(h_{(2)}\right)=\epsilon(h) 1_{H}$, onde $1_{H}$ é a identidade de $H$. Numa notação abreviada:

$$
\cdot(S \otimes i d) \Delta h=\cdot(i d \otimes S) \Delta h=\epsilon(h) 1_{H} .
$$

Assim sendo, dado $h_{(1)} \otimes \cdots \otimes h_{(N)}=h_{(1)(2)} \otimes h_{(1)(2)} \otimes h_{(2)} \cdots h_{(N-1)}=\Delta^{N} h$, em que $h_{(1)(2)} \otimes h_{(1)(2)}$ é o resultado da aplicação de $\Delta$ no primeiro termo de $\Delta^{N-1} h$, aplicamos $\cdot(S \otimes i d) \otimes i d \cdots \otimes i d$ que produz $\epsilon\left(h_{(1)}\right) \otimes h_{(2)} \cdots h_{(N-1)}$, que por 7.337 .34 é igual a $\Delta^{N-2} h$. Em outra palavras, inverte-se, via $S$, o i-ésimo elemento de cada um dos termos somados em $h_{(1)} \otimes \cdots \otimes h_{(N)}$ e pela multiplicação com o termo vizinho cancelam-se ambos (após a soma). 
Um grupo $G$ é um caso particular de álgebra de Hopf. Embora originalmente apenas a multiplicação esteja definida, não há dificuldade em introduzir formalmente a estrutura de espaço vetorial considerando os elementos de $H$ como expressões formais do tipo $\lambda_{1} g_{1}+$ $\lambda_{2} g_{2}+\cdots \lambda_{N} g_{N}, \lambda_{i} \in \mathbb{C}, g_{i} \in G$. A estrutura de Hopf álgebra é completada com $\Delta g=g \otimes g$, $\epsilon(g)=1$ e $S(g)=g^{-1}$. Ocorre que enquanto a multiplicação pela esquerda, $g: a \rightarrow g \cdot a$, fornece uma representação do grupo atuando em si mesmo, não fornece a representação da Hopf álgebra. A representação é fornecida pela ação ou representação adjunta $A d_{g}: a \rightarrow g a g^{-1}$. Mais geralmente, uma representação de $H$ atuando sobre si mesma é dada por $A d_{h}: f \rightarrow h_{(1)} f S\left(h_{(2)}\right), h, f \in H$. A ação adjunta exemplifica que há na verdade mais de uma maneira de uma Hopf álgebra atuar em outras estruturas algébricas, em verdade, inúmeras. Ocorre que a $C^{*}$-álgebra das funções do espaço-tempo transforma-se segundo uma $H$-módulo álgebra pela ação de um grupo.

De fato, observamos que todas as transformações num dado espaço topológico $X$, como 6.3, atuam na $C^{*}$-álgebra das funções de $X, C(X)$, segundo $h \triangleright f(x) \cdot g(x)=(h \triangleright f(x))$. $(h \triangleright g(x))$ e $h \triangleright 1=1$, ou seja, $\Delta h=h \otimes h$ e $\epsilon(h)=1$. As transformações infinitesimais de um grupo de Lie em $X$ (5.34), por sua vez, atuam segundo $\Delta X=X \otimes 1+1 \otimes X, \epsilon(X)=0$. Quando $\tau \Delta h=\Delta h$, onde $\tau(a \otimes b)=b \otimes a$, dizemos que a Hopf álgebra é cocomutativa. Esta observação sugere que esta é a propriedade fundamental de um grupo que atua no espaço comutativo.

Foi proposto na literatura um exemplo de procedimento para mapear a álgebra de Lie de Poincaré numa Hopf álgebra ao quantizar a álgebra de funções do espaço-tempo Agostini et al. (2004). Este foi especificamente aplicado na seguinte álgebra: $\left[x^{j}, x^{0}\right]=i \lambda x^{j}$, $\left[x^{i}, x^{j}\right]=0$, chamada de espaço-tempo $\kappa$-Minkowski $(\kappa=1 / \lambda)$, cuja $C^{*}$-álgebra denotamos $C_{\kappa}$. Este procedimento, contudo, exibe o mesmo tipo de não unicidade que o procedimento de quantização das observáveis clássicas, ou seja, é essencialmente dependente da regra $f(x) \rightarrow \mathcal{Q}(f(x))$. Dado um conjunto de relações de comutação entre coordenadas, a quantização de Weyl 6.27 é um exemplo de regra de quantização, mas não é único. A quantização de Weyl é caracterizada por $e^{i k x} \rightarrow e^{i k \hat{x}}$, mas podemos considerar alternativas como a regra $\Omega_{R}: e^{i k x} \rightarrow e^{i \vec{k} \hat{\vec{x}}} e^{-i k_{0} \hat{x}_{0}}$, ou $\Omega_{S}: e^{i k x} \rightarrow e^{-i k_{0} \hat{x}_{0} / 2} e^{i \vec{k} \hat{\vec{x}}} e^{-i k_{0} \hat{x}_{0} / 2}$. Para cada um 
destes, o $\kappa$-Minkowski implica nas seguintes regras:

$$
\begin{gathered}
\Omega_{R}\left(e^{i p x}\right) \Omega_{R}\left(e^{i q x}\right)=\Omega_{R}\left(e^{\left.i\left(\vec{p}+\vec{q} e^{-\lambda p_{0}}\right) \vec{x}-i\left(p_{0}+q_{0}\right) x_{0}\right)}\right. \\
\Omega_{S}\left(e^{i p x}\right) \Omega_{S}\left(e^{i q x}\right)=\Omega_{S}\left(e^{i\left(\vec{p} e^{\frac{\lambda}{2} q_{0}}+\vec{q} e^{-\frac{\lambda}{2} p_{0}}\right) \vec{x}-i\left(p_{0}+q_{0}\right) x_{0}}\right),
\end{gathered}
$$

que permite uma representação funcional via produto estrela 6.33. Como discutido na vizinhança de 6.34, a cada $C^{*}$ álgebra corresponde uma versão do cálculo integral com ela compatível, no $\kappa$-Minkowski, independentemente da prescrição de quantização, podemos adotar:

$$
\int \Omega_{R, S}(f)=\int d^{4} x f
$$

A ideia de Agostini et al. (2004) é que cada um dos geradores infinitesimais da álgebra de Lie $X_{i}$ na álgebra comutativa dá origem a geradores $X_{i}^{(H)}$ na álgebra não comutativa dados por

$$
X_{i}^{(H)} \Omega_{R, S}\left(e^{i k x}\right)=\Omega_{R, S}\left(X_{i} e^{i k x}\right) .
$$

Uma consequência de 7.39 , dado 7.38 é que

$$
\int X_{i}^{(H)} \Omega_{R, S}(f)=0
$$

propriedade análoga à invariância da medida de integração com respeito à ação do grupo em 4 do capítulo anterior. Outra consequência é que as relações de comutação entre os geradores permanecem inalteradas. Aplicando-se essa regra aos geradores de translação $P_{\mu}$, os autores obtiveram que para $\Omega_{R}$ :

$$
\begin{gathered}
\Delta P_{0}^{R}=P_{0}^{R} \otimes 1+1 \otimes P_{0}^{R} \\
\Delta P_{j}^{R}=P_{j}^{R} \otimes 1+e^{-\lambda P_{0}^{R}} \otimes P_{j}^{R}
\end{gathered}
$$

enquanto que para $\Omega_{S}$ :

$$
\begin{gathered}
\Delta P_{0}^{S}=P_{0}^{S} \otimes 1+1 \otimes P_{0}^{S} \\
\Delta P_{j}^{S}=P_{j}^{S} \otimes e^{\frac{\lambda}{2} P_{0}^{S}}+e^{\frac{\lambda}{2} P_{0}^{S}} \otimes P_{j}^{S}
\end{gathered}
$$

Para os geradores de rotação, em ambos os casos $\Omega_{R, S}$ :

$$
\Delta M_{j}=M_{j} \otimes 1+1 \otimes M_{j}
$$

exatamente como no caso clássico. 
Os mesmos autores observaram que 7.39 aplicado aos geradores de Boost não permite $\Delta N_{j}^{R, S} \in H \otimes H$, de modo que uma definição alternativa se mostra necessária, as hipóteses alternativas foram que as relações de comutação entre $M_{j}$ e $N_{j}$ permanecem inalteradas. Assim como 6, assume-se que a álgebra gerada pelos geradores de translação espaço-temporal é um ideal, além disso, $N_{j}$, assim como no caso relativístico, transformase como um vetor, o que restringe as relações de comutação entre $N_{j}$ e $P_{j}$, por raciocínio análogo ao aplicado no footnote 12 na subseção 6.4

$$
\begin{gathered}
{\left[M_{i}, P_{0}\right]=i A(P) P_{j}} \\
{\left[N_{j}, P_{l}\right]=i \lambda^{-1} B(P) \delta_{i j}+i \lambda C(P) P_{j} P_{i}+i D(P) \epsilon_{j l m} P_{m}}
\end{gathered}
$$

onde $A-D$ são funções algébricas arbitrárias de $P_{0}$ e $\vec{P}^{2}$, que permite expressar $N_{j}$ como: $N_{j} \Omega(\phi(x))=\Omega\left(\left[i x_{0} A\left(-\partial_{x}\right) \partial_{j}+\lambda^{-1} x_{j} B\left(-i \partial_{x}\right)-\lambda x_{l} C\left(-i \partial_{x}\right) \partial_{l} \partial_{j}-i \epsilon_{j k l x_{k}} D\left(-i \partial_{x}\right) \partial_{l}\right] \phi(x)\right)$

trabalhando em cima do requerimento $\Delta N_{j}^{R, S} \in H \otimes H$, os autores restringem $A-D$ e encontram:

$$
\begin{array}{r}
N_{j}^{R} \Omega_{R}(f)=\Omega_{R}\left(\left[i x_{0} \partial_{j}+x_{j}\left(\frac{1-e^{2 i \lambda \partial_{0}}}{2 \lambda}-\frac{\lambda}{2} \nabla^{2}\right)-\lambda x_{l} \partial_{l} \partial_{j}\right] f\right) \\
N_{j}^{S} \Omega_{S}(f)=\Omega_{S}\left(\left[i x_{0} \partial_{j}+x_{j}\left(\frac{\sinh \left(i \lambda \partial_{0}\right)}{\lambda}+\frac{\lambda}{2} \nabla^{2}\right)+\frac{\lambda}{2} x_{l} \partial_{l} \partial_{j}\right] e^{i \frac{\lambda}{2} \partial_{0}} f\right)
\end{array}
$$

A Hopf álgebra resultante no caso $\Omega_{S}$ é:

$$
\begin{gathered}
{\left[P_{\mu}^{S}, P_{\nu}^{S}\right]=0} \\
{\left[M_{j}, M_{k}\right]=i \epsilon_{j k l} M_{l} \quad\left[N_{j}, M_{k}\right]=i \epsilon_{j k l} N_{l} \quad\left[N_{j}, N_{k}\right]=-i \epsilon_{j k l} M_{l}} \\
{\left[M_{j}, P_{0}^{S}\right]=0 \quad\left[M_{j}, P_{k}^{S}\right]=i \epsilon_{j k l} P_{l}^{S}} \\
{\left[N_{j}, P_{0}^{S}\right]=i e^{\frac{\lambda}{2} P_{0}^{S}} P_{i}^{S}} \\
{\left[N_{j}, P_{k}^{S}\right]=i e^{-\frac{\lambda}{2} P_{0}^{S}}\left[\left(\frac{\sin \left(\lambda P_{0}^{S}\right)}{\lambda}+\frac{\lambda}{2} P_{j}^{S} P_{k}^{S}\right)\right]}
\end{gathered}
$$

enquanto

$$
\Delta\left(N_{j}\right)=N_{j} \otimes 1+e^{-\lambda P_{0}^{S}} \otimes N_{j}+\lambda \epsilon_{j k l} e^{-\frac{\lambda}{2} P_{0}^{S}} P_{j}^{S} \otimes M_{k}
$$

Uma observação relevante aqui é que apenas as relações de comutação entre o momento e demais geradores são modificadas daquelas da álgebra de Lie de Poincaré. Ainda continua 
a existir uma álgebra comutativa de geradores de translação espacial e temporal, álgebra esta que por construção é um ideal. Esta álgebra possui como subálgebra a álgebra de Lie do grupo de rotações $S O(3)$, Além disso, $\left[M_{j}, P_{0}^{S}\right]=0$ e existe um Casimir pertencente a álgebra gerada por energia e momento:

$$
C\left(P^{S}\right)=\cosh \left(\lambda P_{0}^{S}\right)-\frac{\lambda^{2}}{2} \overrightarrow{P S}^{2}
$$

Outra observação importante é que se $X_{i}$ é uma realização da álgebra acima no espaço de Hilbert, $X_{i} \otimes I+I \otimes X_{i}$ não é (em particular a representação de Fock 7.10): Observe 7.54. Temos que:

$$
\left[X_{i} \otimes I+I \otimes X_{i}, X_{j} \otimes I+I \otimes X_{j}\right]=\left[X_{i}, X_{j}\right] \otimes I+I \otimes\left[X_{i}, X_{j}\right]
$$

enquanto:

$$
e^{i \frac{\lambda}{2}\left(P_{0}^{S} \otimes I+I \otimes P_{0}^{S}\right)}=e^{i \frac{\lambda}{2} P_{0}^{S}} \otimes e^{i \frac{\lambda}{2} P_{0}^{S}}
$$

A ideia dos autores é que a física do campo escalar relativístico é modificada pela introdução de uma ação invariante pela ação da álgebra de Hopf e que é postulada da forma:

$$
S[\phi(x)]=\int \phi(x)\left(\square_{\lambda}-M^{2}\right) \phi(x),
$$

em que $\phi \in C_{\kappa}, \square_{\lambda} \in H$. Ocorre que, uma vez que $X_{i}^{(H)}$ é da forma 7.39 para algum $X_{i}$ autoadjunto no produto interno $(f, g)=\int d^{4} x f^{*} g, X_{i}$ é autoadjunto no produto $(f, g)=\int f^{*} g$, dado pela integral 7.38 , assim sendo, a ação é invariante por $\phi(x) \rightarrow$ $\phi(x)+i \epsilon X_{i}^{(H)} \phi(x)$ se:

$$
\int \phi(x)\left[\square_{\lambda}, X_{i}^{(H)}\right] \phi(x)=0
$$

7.4.2 Uma prescrição alternativa para a física quântica não relativística e/ou no espaço não comutativo: a prescrição $\pi_{\mathfrak{g}}$

Apresentamos aqui a primeira versão da prescrição original desta tese para formular a física partindo de uma estrutura algébrica que se propõe a substituir o grupo de Poincaré. Evitamos aqui o paradigma usual da física quântica de que tudo deriva de um princípio variacional tal como 7.60 e 6.34

Uma vez que a Hopf álgebra engloba o conceito de grupo e mesmo o conceito de álgebra de Lie, uma hipótese abrangente é postular que a física é invariante pela ação de uma 
Hopf álgebra nas observáveis, mas seguindo uma caminho diferente de 7.60 para definir a física. O nosso ponto de partida é construção $\boldsymbol{G N S}$ da teoria das $C^{*}$-álgebras. A Construção GNS é o que assegura que o problema de representação das $C^{*}$-álgebras tem sempre solução, desde que as operações nela definidas tenham as propriedades requeridas na definição (vizinhança de 6.3). A ideia fundamental é que para todo estado $\omega: \mathcal{A} \rightarrow \mathbb{C}$, funcional linear positivo definido, ou seja $\omega\left(A^{*} A\right) \geq 0$, corresponde uma representação $\pi_{\omega}: \mathcal{A} \rightarrow \mathcal{B}\left(\mathcal{H}_{\omega}\right)$, sendo $\mathcal{B}\left(\mathcal{H}_{\omega}\right)$ a álgebra dos operadores limitados no espaço de Hilbert $\mathcal{H}_{\omega}$, de tal modo que $\mathcal{H}_{\omega}$ contenha um vetor cíclico $\Psi_{\omega}$, ou seja, tal que a aplicação, em $\Psi_{\omega}$, dos operadores da representação gere todos os estados do espaço de Hilbert (mais exatamente, um subespaço denso) e de modo que $\omega(A)=\left(\Psi_{\omega}, \pi_{\omega}(A) \Psi_{\omega}\right)$. Além disso, qualquer outra representação $\pi$ no espaço de Hilbert $\mathcal{H}_{\pi}$ com um vetor cíclico $\Psi$ que satisfaça $\omega(A)=$ $(\Psi, \pi(A) \Psi)$ seja unitariamente equivalente a $\pi_{\omega}$, isto é, existe uma transformação unitária $U: \mathcal{H}_{\pi} \rightarrow \mathcal{H}_{\omega}$ tal que:

$$
U \pi(A) U^{-1}=\pi_{\omega}(A), \quad U \Psi=\Psi_{\omega}
$$

A construção GNS é de extrema importância na matemática e na física. É a base, por exemplo, do teorema de Von Neumann, que mostra que todas as representações irredutíveis da álgebra de Heisenberg são unitariamente equivalentes. A ideia da construção GNS é que a própria $C^{*}$-álgebra é um espaço de Hilbert, a menos de um produto interno a ser definido. Este pode ser tomado $(A, B)=\omega\left(A^{*} B\right), \omega$ um estado qualquer. O produto interno é positivo definido pela propriedade de positividade do estado, mas $(A, A)=0$ não implica necessariamente $A=0$, propriedade do produto interno. Define-se então o conjunto

$$
J=\left\{A \in \mathcal{A}, \omega\left(B^{*} A\right)=0, \forall B \in \mathcal{A}\right\}
$$

que é tal que $\mathcal{A} \cdot J \subseteq J$, propriedade que faz de $J$ um ideal à esquerda (compare com a propriedade 6 de $\mathcal{P}_{\mathcal{N}}$ ), os estados do espaço de Hilbert passam a ser as classes de equivalência definidas pelo conjunto $J$, ou seja, quaisquer dois elementos diferindo por um elemento de $J$ são considerados equivalentes: $[A]=A+B, B \in J$, o que define o chamado espaço quociente $\mathcal{A} / J$. Assim sendo:

$$
\pi_{\omega}(A)[B]=[A B]
$$

Desse modo, a existência das representações de $\mathcal{A}$ se resume à existência dos estados, ou 
funcionais positivo-definidos, mas isso é assegurado pela existência dos funcionais multiplicativos das $C^{*}$-álgebras comutativas 6.4. De fato, cada elemento de $\mathcal{A}$ que comuta com sua adjunta (elemento normal) gera uma subálgebra comutativa com funcionais multiplicativos. Existem elementos normais em qualquer $C^{*}$-álgebra, pois todo elemento de $\mathcal{A}$ pode ser reescrito como a soma de operadores normais: $A=\frac{\left(A+A^{*}\right)}{2}+\frac{\left(A-A^{*}\right)}{2}$. Como argumentado na seção 6.2, um funcional $\omega$ é positivo-definido se e somente se for limitado $(\omega(A) \leq C\|A\|)$, o que equivale a ser contínuo, e $\|\omega\|=\omega(1)(\|\omega\|=C)$, e o já comentado teorema de Hahn-Banach diz que podemos estender funcionais limitados num espaço de Banach para todo espaço mantendo a norma (isto é, estender de modo contínuo). Assim sendo, a extensão dos funcionais multiplicativos conclui a prova da existência das representações. A norma das $C^{*}$-álgebras participa do processo assegurando a existência dos estados. A continuidade do produto com respeito à norma $(\|A \cdot B\| \leq\|A\| \cdot\|B\|)$, implica que os elementos da álgebra são realizados como operadores limitados. Ao remover a norma da definição da álgebra, temos o que se chama de *-álgebra, que pode ser realizado de modo análogo, resultando agora em operadores não limitados, mas resta a questão da existência dos estados que deve ser tratada caso a caso. Como discutido em 5.2 .3 , existem diversos tipos de representações de grupos, tais classificações se aplicam a qualquer representação de estrutura algébrica por transformações lineares num espaço vetorial. Ocorre que os mais diversos tipos de estados se traduzem nos mais diversos tipos de representações.

Podemos aplicar uma versão da construção GNS que produz uma representação da álgebra de Hopf no espaço de Hilbert de modo a satisfazer a regra de Leibniz 7.31. Mas a multiplicação pela esquerda, como comentado, não fornece uma representação. Para fazêlo, temos que considerar a Hopf álgebra dual, $H^{*}$ : o conjunto dos funcionais lineares em $H:\langle\phi, h\rangle \rightarrow \mathbb{C}, \phi \in H^{*}, h \in H$. Esta pode ser transformada numa Hopf álgebra com estrutura induzida daquela em $H:\langle\phi \psi, h\rangle=\langle\phi \otimes \psi, \Delta h\rangle,\langle\Delta \psi, g \otimes h\rangle=\langle\psi, g \cdot h\rangle$, $\langle 1, h\rangle=\epsilon(h)$ e $\epsilon(\psi)=\langle\psi, 1\rangle$.

Podemos então definir a ação de $H$ no seu espaço dual $H^{*}$, que o transforma numa $H$-modulo álgebra, fornecendo uma representação chamada de corregular à esquerda $R^{*}: H^{*} \rightarrow H^{*}:$

$$
\left\langle R_{g}^{*}(\phi), h\right\rangle=<\phi_{(1)}, h>\cdot<\phi_{(2)}, g>=<\phi, h g>
$$

Para proceder na construção, assim como nas $C^{*}$-álgebras, necessitamos de uma involução 
* : $H \rightarrow H$, caracterizada pelas propriedades $(A+B)^{*}=A^{*}+B^{*},(\lambda A)^{*}=\bar{\lambda} A^{*} \mathrm{e}$ tal que $(A B)^{*}=B^{*} A^{*}$ e $\left(A^{*}\right)^{*}=A$. Precisamos, na verdade, apenas assegurar que essas propriedades sejam compatíveis com a involução $X^{*}=X$, pois as transformações infinitesimais geram subgrupos de um parâmetro que são mapeadas em transformações unitárias da forma 5.44. Além disso, compatíveis com a estrutura de Hopf álgebra segundo: $\Delta h^{*}=(\Delta h)^{* \otimes *}, \epsilon\left(h^{*}\right)=\overline{\epsilon(h)}$ (uma vez que $\epsilon$ é um funcional linear limitado e satisfaz $\epsilon(1)=1$ e a involução é caracterizada por $\omega\left(A^{*}\right)=\overline{\omega(A)}$ para todos os funcionais com essa propriedade). Destas, pela unicidade da antípoda, decorre $(S \circ *)^{2}=i d$.

Analogamente ao que ocorre nas $C^{*}$-álgebras, $H^{*}$ é um espaço de Hilbert, a menos de um produto interno a ser definido. Este produto interno deve ser tal que $(\phi, h \triangleright \psi)=\left(h^{*} \triangleright \phi, \psi\right)$. A própria teoria das Hopf álgebras fornece uma candidato chamado de integral à direita, $\int: H^{*} \rightarrow \mathbb{C}$, definido pela propriedade:

$$
\left(\int \otimes i d\right) \circ \Delta=1_{H^{*}} \cdot \int
$$

Analogamente, define-se a integral à esquerda, ambas as integrais são únicas, a menos de um fator multiplicativo. Observe que no caso da Hopf álgebra ser um grupo de Lie, $H^{*}$ é o conjunto das funções definidas na variedade do grupo, a propriedade 7.66 é equivalente à invariância da integral destas com respeito à ação do grupo por translações à direita, $\phi\left(g_{1}\right) \rightarrow \phi\left(g_{1} g_{2}\right):$

$$
\int R_{g_{1}} \phi=\int \phi_{(1)}<\phi_{(2)}, g_{1}>=\epsilon\left(g_{1}\right) \int \phi=\int \phi
$$

propriedade que vincula a escolha da medida de integração na variedade do grupo. A integral à esquerda não é necessariamente equivalente à integral à direita, na teoria dos grupos de Lie, por exemplo, as medidas invariantes por translações à direita, $\phi\left(g_{1}\right) \rightarrow$ $\phi\left(g_{1} g_{2}\right)$, são também invariantes por translações à esquerda, $\phi\left(g_{1}\right) \rightarrow \phi\left(g_{2} g_{1}\right)$, nos grupos compactos. A integral à direita se relaciona com a ação corregular à esquerda segundo:

$$
\int(\phi \triangleright g)^{*} f=\int g^{*}\left(\phi^{*} \triangleright f\right)
$$

Definimos então o ideal $J_{H^{*}}$ :

$$
J_{H^{*}}=\left\{f \in H^{*} / \int g^{*} f=0, \forall g \in H^{*}\right\}
$$


que define o espaço de Hilbert $H^{*} / J_{H^{*}}$. Decorre que transformamos o espaço de Hilbert numa álgebra não comutativa, no qual atua a Hopf álgebra segundo as regras em que atua na $C^{*}$-álgebra das funções do espaço-tempo.

O que decorre da discussão acima é que dispomos de duas estratégias de representação de uma Hopf algebra no espaço de Hilbert. A primeira utilizando-se de funcionais lineares na própria Hopf algebra $H$, onde desconsideramos completamente a estrutura de coproduto (até um momento posterior que vamos discutir), e outra encontrando funcionais em $H^{*}$ tais como a integral, de modo a reproduzir a regra de Leibniz. Para ambos os métodos, vale a noção de representaçõa irredutível e o lemma de Schur. As representações do tipo GNS tem em comum o fato de possuirem um vetor cíclico, aquele na qual a atuação de todos os elementos da álgebra gera um subespaço denso no espaço de Hilbert onde se representa a álgebra. Numa representação irredutível, todas os vetores são cíclicos.

Feita essa discussão, enunciemos a referida prescrição:

Prescrição $1\left(\pi_{\mathfrak{g}}\right)$. Seja $\mathfrak{p}_{\mathcal{N}}$ uma Hopf álgebra com geradores $X_{i}$ e $\mathcal{P}_{\mathcal{N}}$ o grupo gerado pelos subgrupos de um parâmetro da forma $e^{-i X_{i} \theta_{i}}, t \in \mathbb{R}$. Seja $\pi_{\lambda^{\prime}}: \mathfrak{p}_{\mathcal{N}} \rightarrow O(\mathcal{D})($ em que $O(\mathcal{D})$ são os operadores essencialmente autoadjuntos no dominio invariante $\mathcal{D}$ ) a representação irredutivel de $H$ (por qualquer dos métodos acima) com conjunto de autovalores dos operadores de Casimir $\lambda^{\prime}=\left(\lambda_{0}, \lambda_{i}\right)$. Seja $\mathcal{U}_{\lambda^{\prime}}^{\mathcal{N} \mathcal{P}}$ a representação de $\mathcal{P}_{\mathcal{N}}$ gerada por elementos da forma $e^{-i \pi_{\lambda^{\prime}}\left(X_{i}\right) t}$. A representação de $\mathcal{P}_{\mathcal{N}}$ que descreve a teoria livre é obtida de 7.8 pela a substituição:

$$
\mathcal{U}_{\lambda} \rightarrow \mathcal{U}_{\lambda^{\prime}}^{\mathcal{N} \mathcal{P}}
$$

para algum conjunto $\lambda^{\prime}$. Essa prescrição nos leva à seguinte representação do grupo $\mathcal{P}_{\mathcal{N}}$ para descrever a radiação segundo uma teoria livre:

$$
U_{F}^{\mathcal{N} \mathcal{P}}=\sum_{N=0}^{\infty} \oplus\left(\mathcal{U}_{\lambda^{\prime}}^{\mathcal{N} \mathcal{P} \otimes N}\right)_{S}
$$

Esta prescrição ainda não está completa, falta ainda definir a regra que leva o conjunto de autovalores de Casimir de Poincaré, $\lambda$, para o conjunto de autovalores de Casimir da Hopf álgebra $\lambda^{\prime}$. Tratemos disso mais adiante. Por agora, observemos que 7.71 leva às equações fundamentais da inflação não comutativa segundo a discussão na seção 7.2 . Observe que estamos aqui substituindo uma representação do grupo de Poincaré associada à teoria livre por um representação $\pi_{\mathfrak{g}}$ do grupo $\mathcal{P}_{\mathcal{N}}$ (conforme discutido em 7.3). 
Os geradores dos subgrupos de um parâmetro de 7.71, contudo, não necessariamente produzem uma representação da mesma Hopf álgebra $\mathfrak{p}_{\mathcal{N}} \cdot$ Em outras palavras, se $H$ é o gerador de translações temporais da álgebra cujo Casimir é a relação de dispersão inflacionária, 7.10 pode não ser. Essa condição não ser satisfeita, enquanto 7.71 descreve a teoria livre, implicaria que não existe uma noção única de relação de dispersão na física. Esse tipo de representação ser aplicado em física pode significar que um estado de duas ou mais partículas que é estável num referencial pode ser instável em outro referencial, conforme discussão no footnote 6 do capítulo anterior. Ou ainda, uma matriz de espalhamento que conectasse estados de uma partícula com estados de duas ou mais partículas não conservaria energia em todos os referenciais.

Para analisar se este é o caso, precisamos considerar restrições adicionais na estrutura algébrica dos geradores das simetrias. Estas restrições são justamente sobre a forma da relação de dispersão que produz a inflação. Podemos considerar o ansatz 7.21 dos autores originais, mas apresentamos aqui uma extensão sistemática da classe de relações de dispersão inflacionárias, um dos resultados originais desta tese. 
Capítulo 7. A Inflação Algébrica I: 
Capítulo 8

\section{A Inflação Algébrica II: \\ Uma nova prescrição para definir a física inflacionária não relativística}

\subsection{Introdução}

No capítulo anterior, escolhemos definir a fisica, não por uma deformação da ação dos campos, como usualmente feito na literatura, ou mesmo em tratamentos não relativísticos da inflação como em Martin e Brandenberger (2001), Brandenberger (2003) e Martin e Brandenberger (2003), mas por um esquema de representação de simetrias generalizado que parte de uma Hopf álgebra. Propusemos a prescrição 1 para definir a física. Observamos que 7.71 leva às equações fundamentais da inflação não comutativa segundo a discussão na seção 7.2. Observe que estamos aqui substituindo uma representação do grupo de Poincaré associada à teoria livre por um representação $\pi_{\mathfrak{g}}$ do grupo $\mathcal{P}_{\mathcal{N}}$ (conforme discutido em 7.3). Contudo, os geradores dos subgrupos de um parâmetro de 7.71 não necessariamente produzem uma representação da mesma Hopf álgebra $\mathfrak{p}_{\mathcal{N}}$. Essa condição não ser satisfeita, enquanto 7.71 descreve a teoria livre, implicaria que não existe uma noção única de relação de dispersão na física acarretando em problemas conceituais como as descritas no final do capítulo anterior. Isto é, uma física que se propõe ser invariante pela ação de geradores que satisfazem uma álgebra de Hopf $\mathfrak{p}_{\mathcal{N}}$, não necessariamente pode ser descrita por uma representação da forma 7.71. Para determinar se os geradores de qualquer representação $\pi_{\mathfrak{g}}$ do grupo $\mathcal{P}_{\mathcal{N}}$ que realiza a inflação 
não comutativa realizam também a mesma Hopf álgebra, precisamos investigar restrições adicionais na estrutura algébrica que se propõe a substituir a álgebra de Lie de Poincaré. Estas restrições são justamente sobre a forma da relação de dispersão que produz a inflação. Podemos considerar o ansatz 7.21 dos autores originais, mas apresentamos aqui uma extensão sistemática da classe de relações de dispersão inflacionárias, um dos resultados originais desta tese.

Obtemos que 7.71 não pode realizar a mesma álgebra de geradores $\mathfrak{p}_{\mathcal{N}}$ para nenhuma escolha inflacionária de relações de dispersão compatíveis com o requerimento de mínimo número de e-folds. Em particular, provamos que não existe grupo de Lie (como o grupo de Poincaré) que possa realizar a inflação não comutativa, necessariamente temos que recorrer a estruturas mais gerais, como as Hopf álgebras, por exemplo. Propomos aqui uma nova prescrição para definir a física não relativística que restringe a ocorrência de representações do grupo $\mathcal{P}_{\mathcal{N}}$ àquelas que realizam a mesma álgebra de geradores $\mathfrak{p}_{\mathcal{N}}$. Se acompanhada de uma regra geral para deformar a álgebra de Lie de Poincaré numa Hopf álgebra que a deforma em função da informação que define um espaço não comutativo (como na subseção 7.4.1), temos uma prescrição alternativa àquela que existe na literatura para definir a física no espaço não comutativo, prescrição essa com vantagens conceituais como evitar por construção problemas com renormalização e unitariedade (dificuldade que geralmente ocorre na quantização de lagrangianas gerais, inclusive em teorias não comutativas obtidas da prescrição 6.34), ou a existência de uma noção única de simetrias aplicável a todos o campos, que demonstramos por 6.73 não ser satisfeita pela prescrição dominante na literatura 6.34.

\subsection{Condições sobre $f(E)$ análogas às condições de rolamento lento da inflação dirigida por campo escalar}

Como discutimos no capítulo 3 , na realização da inflação no contexto de campos escalares, não é a forma específica do potencial que leva à inflação, mas a validade das condições de rolamento lento, 3.58 e 3.60 . Essas condições implicam que existe uma grande variedade de condições iniciais no espaço de configurações do campo que produzem a quantidade 
necessária de inflação, o número mínimo de e-folds 3.85 .

Podemos nos perguntar se a mesma situação ocorre na inflação não comutativa: Assumindo a forma das equações do modelo, 7.19 e 7.20 , existem desigualdades sobre $f(E)$ em 7.17 que levam a uma inflação bem sucedida no limite homogêneo? Por homogêneo, queremos dizer sem considerar vínculos no espectro de perturbações gerado (comentaremos sobre o problema adiante) e por bem sucedida, queremos dizer que produz o número mínimo de e-folds e não tenha problema de saída graciosa.

A escolha $f=1+(\lambda E)^{\alpha}$ feita em Alexander et al. (2003) não é tão arbitrária quanto pode parecer à primeira vista. De fato, a substituição dessa relação no denominador de 7.19 leva a uma equação de estado $w=p / \rho$ constante no limite de elevadas temperaturas se o espectro de $\rho(E, T)$ atinge seu máximo a valores de energia cada vez maiores para valores de temperatura cada vez maiores e de tal modo que a principal contribuição da integral de 7.18 na energia venha de regiões com energias arbitrariamente elevadas, como no espectro de Planck usual. Essa aproximação é a justificação da escolha.

Como demonstrado numericamente em Alexander et al. (2003), a hipótese de picos de $\rho(E, T)$ para valores arbitrariamente maiores de energia falha para a escolha de $f(E)$ feita quando se restringe $\alpha$ no intervalo inflacionário. Para elevadas temperaturas, temos, ao invés disso, um pico saturado (veja Fig 1 em Alexander et al. (2003)). Demonstramos a seguir uma extensão sistemática da classe de funções $f(E)$ que leva à inflação, extensão essa original desta tese, começando por versar requerimentos cosmológicos em desigualdades sobre $f(E)$.

\subsubsection{Condição de termodinâmica aceitável e limite de baixas energias}

Podemos esperar que para um $f(E)$ genérico, as equações 7.19 7.20 produzam uma curva genérica para a equação de estado $w=\frac{p}{\rho}$ como mostrado na Fig. 8.1 , com $w \sim 1 / 3$ para baixas temperaturas e um período de aceleração $-1 \leq w \leq-1 / 3$ para algum intervalo de altas temperaturas entre $T_{1}$ e $T_{2}$.

Na equação 7.18 $\frac{E^{2}}{f^{3}}\left|1-\frac{E f^{\prime}}{f}\right|=\left|p^{2} \frac{d p}{d E}\right|=\left|\frac{1}{3} \frac{d\left(p^{3}\right)}{d E}\right|$ é proporcional ao número de estados de uma partícula acessíveis por intervalo de energia (que decorre de $\Omega(E) d E=\Omega(p) d p)$. Disso, observamos o primeiro requerimento físico: algumas escolhas de relação de dispersão levam a expressões divergentes de densidade de energia e pressão no equilíbrio 


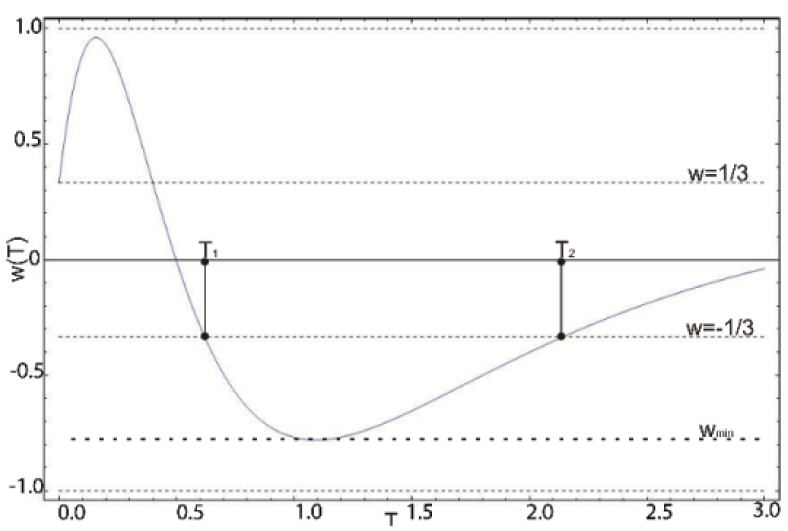

Figura 8.1: Generic graphic of $w(T)$ versus $T$.

termodinâmico, porque levam a muitos estados de partícula por intervalo de energia. Requeremos adicionalmente que $f(E \rightarrow 0) \rightarrow 1$ para baixas energias, ou seja, a relação de dispersão seja usual. Adicionalmente, requeremos que $w(T \rightarrow 0)=1 / 3$ para a equação de estado usual de baixas energias. Essas condições são na verdade relacionadas e, com a condição adicional $\lim _{E \rightarrow 0} E f^{\prime}(E)=0$, temos o primeiro conjunto de condições sobre a relação de dispersão (para a demonstração, veja o apêndice A do nosso artigo ?):

Teorema 5. Se $\frac{1}{f^{3}}\left|1-\frac{f^{\prime} E}{f}\right| \leq C\left(1+E^{k}\right) e \frac{1}{f^{3}} \leq C^{\prime}\left(1+E^{k^{\prime}}\right)$ para constantes reais positivas $C$ e $C^{\prime}$ e para inteiros $k$ e $k^{\prime}, f(E)$ e $f^{\prime}(E)$ contínuos para $E \geq 0$ com $\lim _{E \rightarrow 0} f(E)=1$, and $\lim _{E \rightarrow 0} E f^{\prime}(E)=0$, então as expressões para densidade de energia e presão no equilíbrio térmico são finitas e:

$$
\lim _{T \rightarrow 0} \frac{p(T)}{\rho(T)}=\frac{1}{3}
$$

\subsubsection{Condição de número mínimo de e-folds}

Outra condição que precisa ser satisfeita pela inflação é que tenhamos um número mínimo de e-folds $\mathcal{N}, 3.85$. A estimativa desse número depende do problema em consideração (flatness, horizon, etc), O número é $\gtrsim 60$.

Da Fig. 8.1 podemos estimar que

$$
\frac{1}{2} \ln \frac{\rho\left(T_{2}\right)}{\rho\left(T_{1}\right)}<\mathcal{N}<\frac{1}{3\left(1-w_{\min }\right)} \ln \frac{\rho\left(T_{2}\right)}{\rho\left(T_{1}\right)} .
$$

Essa estimativa vem da equação de conservação da energia que leva a $-3\left(1-\frac{1}{3}\right) d \ln a<$ $d \ln \rho<-3\left(1-w_{\text {min }}\right) d \ln a$. Chegamos, por tanto, à conclusão de que para um número 
mínimo de e-foldings $\mathcal{N}$, é suficiente que $\frac{\rho(T 2)}{\rho(T 1)}>\exp 2 \mathcal{N}$. Em particular, é suficiente $\frac{\rho(T 2)}{\rho(T 1)} \rightarrow \infty$. Esse é o caso para modelos de inflação com equação de estado constante no limite de altas energias.

Obtenhamos condições que asseguram equação de estado constante no limite de altas temperaturas (veja apêndice B da nossa publicação Machado e Opher (2012) para demonstração):

Teorema 6. Defina $g=1-\frac{f^{\prime} E}{f}$ com as seguintes propriedades:

1. $g(E \rightarrow 0)=1$ e g é continuamente diferenciável para $E \geq 0$.

2. Existe um número finito $N$ de energias $E_{01}, E_{02}, \ldots E_{0 N}$ tais que $g\left(E_{0 j}\right)=0$ e $\left|\frac{d g\left(E_{0 j}\right)}{d E}\right|>$ 0 .

3. Existe um $\epsilon>0$ tal que $g(E) \leq-\epsilon$ para $E \geq E_{1}$

Sob tais condições,

$$
\begin{aligned}
\lim _{T \rightarrow \infty} w(T)=\frac{1}{3}\left(\int_{0}^{E_{01}} E^{2} / f^{3} d E-\int_{E_{01}}^{E_{02}} E^{2} / f^{3} d E+\ldots\right. \\
\left.\quad+\int_{E_{0 N-1}}^{E_{0 N}} E^{2} / f^{3} d E-\int_{E_{N}}^{\infty} E^{2} / f^{3} d E\right) / \int_{0}^{\infty}|g| E^{2} / f^{3} d E,
\end{aligned}
$$

onde cada integral envolvida converge.

É importante comentar que no trabalho original Alexander et al. (2003), a aproximação

$$
\lim _{T \rightarrow \infty} w(T) \approx \frac{1}{3(1-\alpha)}
$$

é feita ao invés de uma fórmula exata. Adicionalmente, as condições do Teorema 5 são automaticamente satisfeitas.

Adotemos a seguinte convenção:

Definição 1. O conjunto $G_{N}$ consiste de todas as funções g satisfazendo todas as condições do teorema 6 e tendo $N$ raízes.

A despeito de todos os detalhes matemáticos envolvidos nesta e nas demais proposições que constroem a classe de relações de dispersão, a observação relevante aqui é a forma da função $p(E)$, que relaciona a energia com o módulo do momento, que advém dessas condições. 
A primeira condição é relacionada com o limite de baixas energias da relação de dispersão, ou seja, como esperado, $\lim _{E \rightarrow 0} p(E) / E=1$. Os zeros da função $g(E)$ são máximos e mínimos locais do momento como função da energia $\left(\frac{d p}{d E}=0\right)$, i.e., pontos de transição entre modos de pressão positiva e negativa (se $\left|\frac{d g}{d E}\right|>0$ ). A Inflação necessariamente precisa de pressão negativa, $\frac{d p}{d E}<0$ é então necessário, porque o mecanismo de inflação é tal que, quando o universo expande, o comprimento de onda de estados de uma partícula aumenta, o momento diminui em proporção inversa e, para partículas com uma relação de dispersão convencional, na qual $\frac{d p}{d E}>0$, isso implica em diminuição da energia, mas para partículas com $\frac{d p}{d E}<0$ a energia aumenta, levando à pressão negativa. Esse raciocínio assume $p=p(E)$, uma função que associa um único valor de momento (em módulo) para cada energia, o que está implicitamente assumido em 7.17 .

A existência de pontos com $\frac{d p}{d E}=0$ é então necessária para inflação e implica que devemos ter funções $p(E)$ não inversíveis. O número de tais pontos é relacionado com oscilações mais complexas da equação de estado como função da temperatura. A condição 3 é a condição importante aqui, porque implica que $p(E \rightarrow \infty)=0$, que por sua vez leva à existência de um momento máximo abaixo do qual todo nível de energia é mapeado (os autores originais associam isto a uma mínima escala sondável no espaço). A condição 2 implica em um número finito de ramos de energia. Os ramos de energia são regiões conexas do gráfico de pontos $(E,\|p\|)$ onde a equação $p=p(E)$ é inverssível num intervalo de energia que não pode ser estendido de maneira conexa.

\subsubsection{A condição de saída graciosa}

O problema da saída graciosa vem do fato de que se, em alguma vizinhança de $T, \rho(T)$ é uma função diferenciável e inversível, podemos escrever $w=w(\rho)$, a equação de estado para um fluido isentrópico. Para uma métrica FRW, a conservação do tensor energiamomento, $T_{; \nu}^{\nu \mu}=0$, se traduz como $\frac{d(\rho)}{\rho}=-3(1+w) \frac{d a}{a}$ e temos que, se existe um $T_{0}$ nessa vizinhança tal que $\rho_{0}=\rho\left(T_{0}\right)$ e $w\left(\rho_{0}\right)=-1$, a equação anterior pode ser reescrita como $\frac{d \ln \rho}{d \ln a}=-3(1+w(\ln \rho))$, e tem uma única solução $\rho(\ln a)=\rho_{0}$ para $\rho(\ln a)=\rho_{0}$.

Pelo teorema da função inversa, temos que se $\rho(T)$ é uma função continuamente diferenciável de $T$ e para algum valor $T_{0}$ temos que $\left|\frac{d \rho}{d T}\right|>0$, então decorre que $\rho(T)$ é uma função inversível numa vizinhança $\mathcal{U}$ que contém $T_{0}$. Isso significa que se existe tal 
temperatura $T_{0}$ então nessa temperatura o universo nunca deixa a fase acelerada. Como demonstramos (no apêndice C), $\frac{d \rho}{d T}>0$ sempre.

Pode-se esperar do acima exposto que todas as relações de dispersão levando a $\lim _{T \rightarrow \infty} w \in$ $(-1 / 3,-1)$ levam a uma cosmologia aceitável. Isso não é verdade. Precisamos ter $w>-1$ para evitar o problema da saída graciosa, como discutido acima. Não é verdade em geral que $\lim _{T \rightarrow \infty} w>-1$ implica $w(T)>-1$ para todo $T$.

Considere, por exemplo, a função $\theta\left(E, E_{0}, \delta, \alpha\right) \rrbracket$. Esta é uma função infinitamente diferenciável que utilizamos para construir a seguinte função $g$ :

$$
g(E)=1+\theta(E, 0,1,-1.05)+\theta(E, 100,3,3)+\theta(E, 200,3,-3.2)
$$

Esta função corresponde a um momento que primeiro decresce com a energia rumo a zero, então aumenta e depois decresce novamente em direção a zero, e produz o gráfico da Fig. 8.2 que mostra um problema de saída graciosa: existe uma temperatura $T$ tal que $w(T)=-1$, mesmo com uma equação de estado assintótica satisfazendo $w>-1$.

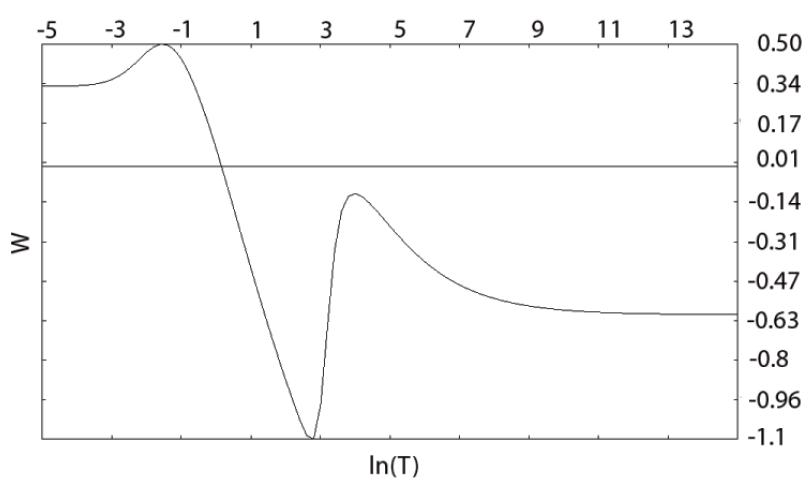

Figura 8.2: $\mathrm{w}(\mathrm{T})$ versus $\ln (\mathrm{T})$ para a função $g$ da Eq. 8.4 Temos nesse exemplo uma equação de estado de alta temperatura no intervalo inflacionário $(w(T \rightarrow \infty) \approx-0.6)$, mas para algum $T$, temos que $w(T)<-1$, levando a um problema de saída graciosa.

Somos levados à próxima questão: Que condições asseguram $w(T)>-1$ para todo $T$ ? Asseguramos isso pelo seguinte teorema (veja o apêndice C de Machado e Opher (2012) para a demonstração):

Teorema 7. Se $g \in G_{1}$, uma vez que tenhamos $\lim _{T \rightarrow \infty} w>-1$, necessariamente teremos $w(T)>-1$ para todo $T$ se $g>-1 / 3$.

1 Que tem valor 0 quando $E<E_{0}$; valor $\alpha$ quando $E>E_{0}+\delta$; e valor $\alpha \int_{-1}^{-1+2 \frac{E-E_{0}}{\delta}} e^{-\frac{1}{1-x^{2}}} d x / I$, com $I=\int_{-1}^{1} e^{-\frac{1}{1-x^{2}}} d x$, quando $0<E-E_{0}<\delta$. Uma transição suave entre dois valores 
Esta é uma condição suficiente para evitar o problema da saída graciosa e não uma condição necessária.

\subsection{O algoritmo numérico}

Não é óbvio como inverter a Eq, 8.2 no intervalo $\left(-\frac{1}{3},-1\right)$ para identificar deformações genéricas de relação de dispersão que levam a uma equação de estado inflacionária a altas temperaturas. Nossa estratégia será postular uma família de um parâmetro $(\alpha)$ de relações de dispersão que sejam asseguradas por construção conter pelo menos um intervalo do parâmetro $\alpha$ onde a inflação ocorre de maneira bem sucedida.

Faremos isso construindo uma sequência de funções $g_{N}, N=1,2, \ldots$ compatíveis com todos os requerimentos considerados até agora e de tal modo que $\lim _{N \rightarrow \infty} w_{N}^{\infty}<-1$ $\left(\lim _{T \rightarrow \infty} w_{N}(T) \equiv w_{N}^{\infty}\right)$ e outra sequência tal que $\lim _{N \rightarrow \infty} w_{N}^{\infty}>-1 / 3$. Interpolaremos continuamente com respeito a um parâmetro $\alpha$ entre dois elementos suficientemente avançados dessas sequências (de tal modo que os elementos satisfaçam a mesma desigualdade que o limite), de um modo compatível com os requerimentos anteriores e tal que $\lim _{T \rightarrow \infty} w$ dependa continuamente com o parâmetro $\alpha$.

Uma dessas sequências é construída utilizando-se do seguinte teorema que assegura $\lim _{N \rightarrow \infty} w_{N}^{\infty}<-1$ para $N$ suficientemente grande (Veja apêndice D de Machado e Opher (2012))

Teorema 8. Suponha uma sequência funcional $g_{N}, N=1,2 \cdots$ tal que $\left(g_{N} \in G_{1}, g_{N}>\right.$ $-\frac{1}{3}$ e $\left.g_{N}\left(\xi_{N}\right)=0\right)$ :

1. $g_{N}(E) \geq 1-E / \xi_{N}, 0 \leq E \leq \xi_{N}$ para todo $N$.

2. $\left|g_{N}(E)\right|<\epsilon_{N}$ para $E \geq \xi_{N}$.

3. $g_{N}<c_{N}, 0<c_{N}<c e\left(c_{N}-1\right) \xi_{N}<k$ para $E<\xi_{N}$.

4. $\epsilon_{N} \rightarrow 0$ quando $N \rightarrow \infty$.

5. $\exists \xi^{\prime}, 0<\xi^{\prime}<\xi_{N}$ tal que $g_{N}<1+\alpha E, \alpha>0$, se $E \leq \xi^{\prime}$

então, $\lim _{N \rightarrow \infty} w_{N}^{\infty}=-\infty$. 
Para construir a outra sequência, utilizamos a seguinte proposição que assegura $\lim _{N \rightarrow \infty} w_{N}^{\infty}>$ $-1 / 3$ para $N$ suficientemente grande (veja apêndice E em Machado e Opher (2012)):

Teorema 9. Suponha uma sequência funcional com $g_{N}(E)\left(g_{N}(E) \in G_{1} \operatorname{com} g_{N}(E)>\right.$ $-1 / 3$ e $\left.g_{N}\left(\xi_{N}\right)=0\right)$ satisfazendo:

1. $g_{N}(E)<1+\alpha E$ para $E<\xi^{\prime}$, para todo $N$ e $0<\alpha<\infty$

2. $g_{N}(E)>1-\beta E$ para algum $0<\beta<\infty$, para $E<\xi^{\prime \prime}<\xi^{\prime}$ e para todo $N$

3. $g_{N}(E)<1 / 3-\epsilon$, para algum $\epsilon>0, E \geq \xi^{\prime}$ e para todo $N$.

4. $\xi_{N} \rightarrow \infty$ quando $N \rightarrow \infty$

5. $g_{N}(E)<-1 / 3+\epsilon_{N}$ para $E>\xi_{N}+\Delta_{N}$, com $\epsilon_{N} \rightarrow 0$ e $\Delta_{N} \rightarrow 0$ quando $N \rightarrow \infty$ , então, para $N$ suficientemente grande nós temos $w_{N}^{\infty}>0$.

As proposições acima ainda funcionam sem a restrição $g_{N}>-\frac{1}{3}$, mas o teoremas anteriores não são suficientes para assegurar saída graciosa da inflação.

Por uma interpolação, nos referimos a uma função $g(\alpha, E)$ com $\alpha \in\left[\alpha_{1}, \alpha_{2}\right]$ tal que $g\left(\alpha_{1}, E\right)=g_{N}(E) \operatorname{com} w_{N}^{\infty}>-1 / 3$ e $g\left(\alpha_{2}, E\right)=g_{N}^{*}(E) \operatorname{com} w_{N}^{* \infty}<-1, g(\alpha, E)$ contínuo (i.e. ||$\left(\alpha_{1}, E_{1}\right)-\left(\alpha_{2}, E_{2}\right)||<\delta$ implica $\left|g\left(\alpha_{1}, E_{1}\right)-g\left(\alpha_{2}, E_{2}\right)\right|<\epsilon$ para qualquer $\epsilon$ e algum

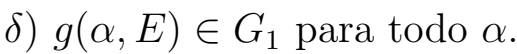

Extremamente importante para este procedimento ser válido em geral é que possamos assegurar continuidade da equação de estado de alta temperatura com respeito ao parâmetro de interpolação $\alpha$. Precisamos ser cuidadosos, em geral, enquanto construindo famílias contínuas de um parâmetro de funções contínuas e esperando que a integral dessas funções seja contínua com respeito ao parâmetro associado. Considere o exemplo envolvendo uma integral em um intervalo ilimitado (exatamente como na Eq. (8.2)

$$
g(x, \alpha)=\left\{\begin{array}{rrr}
\frac{\alpha}{\sqrt{\pi}} e^{-(\alpha x)^{2}} & \text { if } & \alpha>0 \\
0 & \text { if } & \alpha=0
\end{array}\right.
$$

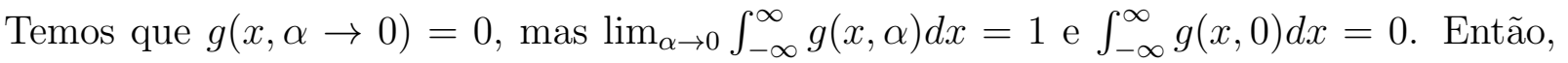
não temos continuidade da integral com respeito ao parâmetro $\alpha$. A definição da classe $G$ de funções torna fácil assegurar a continuidade desejada, mas mesmo na Fig.3 de Alexander et al. (2003) temos um exemplo de descontinuidade da equação de estado de alta 
temperatura com respeito ao parâmetro $\alpha$ (Demonstração do teorema no apêndice $\mathrm{F}$ de Machado e Opher (2012)):

Teorema 10. Suponha $g(E, \alpha)$ uma função limitada $(|g(E, \alpha)|<C$ para todo $\alpha$ e todo $E)$ e diferenciável tal que $g(E, \alpha) \in G_{1}$ para todo $\alpha \in\left[\alpha_{1}, \alpha_{2}\right]$ ( e, como consequência da definição de $G_{1}, \frac{d g(E, \alpha)}{d E}<0$ quando $E=\xi_{N}(\alpha)$ de tal modo que $\left.g\left(E, \xi_{N}(\alpha)\right)=0\right)$, então:

$$
\frac{1}{3} \frac{\int_{0}^{\xi_{N}(\alpha)} E^{2} / f(E, \alpha)^{3} d E-\int_{\xi_{N}(\alpha)}^{\infty} E^{2} / f(E, \alpha)^{3} d E}{\int_{0}^{\infty}|g(E, \alpha)| E^{2} / f(E, \alpha)^{3} d E}
$$

é contínuo com respeito ao parâmetro $\alpha$.

Os últimos três teoremas apenas afirmam que podemos começar com um quase arbitrário palpite inicial para $g(E)$ e, por controle numérico de algum número finito de parâmetros de deformação, construindo uma sequência, podemos construir uma família de um parâmetro de deformações da relação de dispersão que conterá um intervalo do parâmetro $\alpha$ de equações de estado assintóticas inflacionárias (i.e. $w(T \rightarrow \infty)$ conterá na sua imagem o intervalo $(-1 / 3,-1))$, terá uma equação de estado convencional a baixas energias e não terá problema de saída graciosa. Os teoremas de sequências funcionais dão flexibilidade para deformar o palpite inicial ao longo de toda sua extensão. Desde que tenhamos encontrado os extremos da família, digamos $g_{1}(E) \operatorname{com} w(T \rightarrow \infty)>-1 / 3$ e $g_{2}(E) \operatorname{com} w(T \rightarrow \infty)<-1$, se $g_{1}\left(\xi_{1}\right)=0$ e $g_{2}\left(\xi_{2}\right)=0$ para $\xi_{1}<\xi_{2}, d g_{1}(E) / d E<0$ e $d g_{2}(E) / d E<0$ no intervalo $\left(\xi_{1}, \xi_{2}\right)$, então $\alpha g_{1}(E)+(1-\alpha) g_{2}(E)$ com $0 \leq \alpha \leq 1$ satisfaz todos os requerimentos do teorema de continuidade, por exemplo.

\subsection{Uma prescrição alternativa para a física quântica não relativística e/ou no espaço não comutativo: a prescrição $\pi_{\mathcal{A}}$}

Em 7.4.2 propusemos uma prescrição para a construção da teoria livre a partir da construção GNS aplicada às Hopf álgebras. Esta prescrição, embora ainda aplicável apenas a teorias livres, quebra o paradigma usual da física quântica de que tudo decorre de uma ação a ser quantizada. Essencialmente, $\mathcal{P}_{\mathcal{N}}$ é um grupo associado a uma Hopf álgebra e postulamos que uma representação unitária $\pi_{\mathfrak{g}}$ no espaço de Hilbert, a qual satisfaz $\pi_{\mathfrak{g}}\left(g_{1} \cdot g_{2}\right)=\pi_{\mathfrak{g}}\left(g_{1}\right) \cdot \pi_{\mathfrak{g}}\left(g_{2}\right), g_{1}, g_{2} \in \mathcal{P}_{\mathcal{N}}$, descreve a radiação na descrição de teoria livre. Essa prescrição concorda com as equações fundamentais da inflação não comutativa como 
formuladas originalmente. Contudo, como não estamos a princípio lidando com grupos de Lie, não é verdade em geral que toda representação do grupo induz uma representação da mesma álgebra de geradores. Para determinar se a representação 7.71 realiza a mesma álgebra e, por tanto, com mesmo Casimir, necessitávamos de mais informação sobre a classe de relações de dispersão envolvida na inflação não comutativa. Com base na análise das seções anteriores, mostraremos que nenhuma álgebra de Lie pode realizar a inflação não comutativa. Além disso, a representação 7.71, para a classe de relações de dispersão inflacionárias, não preserva a mesma álgebra de geradores de qualquer Hopf álgebra que possa ser utilizada na construção das representações irredutiveis que a compõem. Se a representação 7.71 fizer parte da física, significaria que não existe uma noção única de relação de dispersão no universo, levando a problemas conceituais, como as discutidas no final do capítulo anterior. Uma solução para esse problema seria, assim como é feito na teoria de campos usual (e discutido no capítulo5), restringir, por postulados, a classe de representações de $\mathcal{P}_{\mathcal{N}}$ que ocorre na natureza, para aquelas que preservam a mesma álgebra de geradores. Assim sendo, 7.71 deveria ser substituída por outra representação de $\mathcal{P}_{\mathcal{N}}$. Propomos aqui uma alternativa à 7.71, que pode ainda ser estendida a toda a teoria de campos em interação evitando, por construção, problemas com unitariedade e renormalização que advém da técnica usual de diagramas de Feynmann quando tratamos Lagrangianas gerais como 7.60 e 6.34. Este é o principal resultado desta tese.

\subsubsection{A decomposição na forma integral direta}

No capítulo 5, discutimos a generalização para dimensão infinita do teorema espectral feita por Von Neumann, Teorema 1. Afirmamos que nos delongamos sobre ele por ser uma das bases de toda a teoria de representação de operadores no espaço de Hilbert. Pois bem, partimos deste teorema para deduzir uma importante representação para operadores autoadjuntos no espaço de Hilbert, a forma integral direta.

Sabemos da praxe usual da mecânica quântica que os operadores posição e momento que representam irredutivelmente a álgebra de Heisenberg podem ser (não simultaneamente) 
diagonalizados e postos na forma $x: \psi(x) \rightarrow x \psi(x)$ no espaço de Hilbert $L^{2}(\mathbb{R})$. Esta, contudo, não é a forma geral na qual um operador autoadjunto pode ser diagonalizado, esta forma é válida apenas quando o operador é cíclico e o espectro é igual a $\mathbb{R}$ (footnote 5 na subseção 5.2.1. Vamos discutir essa generalização, mas primeiro faremos um comentário preliminar sobre a integral de Stieltjes (definida no footnote 4 da subseção 5.2.1) e a mais geral integral de Lebesgue.

Quando definimos a integral de Stieltjes, discriminamos dois tipos de conjuntos de $\mathbb{R}$, os da forma $\left\{a_{n}\right\}$ e os da forma $\left(a_{n}, b_{n}\right)$. A coleção de todos esses conjuntos é o que se chama, na teoria da medida, de semi-anel. Uma semi-anel $\mathcal{S}$ é uma coleção de conjuntos que possui o conjunto vazio Ø; se $S_{1}$ e $S_{2} \in \mathcal{S}$, então $S_{1} \cap S_{2} \in \mathcal{S}$; se $S_{0} \subseteq S$ estão em $\mathcal{S}$, então existem $S_{j} \in \mathcal{S}$ disjuntos tais que $S-S_{0}=\sum_{j=1}^{m} S_{j}, m<\infty 2^{2}$. A função $\rho(\lambda)$ é utilizada para definir o que se chama de medida positiva $\sigma$-aditiva em $\mathcal{S}$, isto é $\mu: \mathcal{S} \rightarrow \mathbb{R}^{+}$ de tal modo que $\mu(S)=\sum_{n=1}^{\infty} \mu\left(S_{n}\right)$ sempre que $S=\sum_{n=1}^{\infty} S_{n}$. Na teoria da medida e integração, o problema fundamental é definir toda uma variedade de regras de integração $\int d \mu$ numa classe $\mathcal{F}_{\mu}$ de funções $f: \Omega \rightarrow \mathbb{R}$ que satisfaça $\int d \mu \sum_{n=0}^{\infty} f_{n}=\sum_{n=0}^{\infty} \int d \mu f_{n}$, $f_{n} \in \mathcal{F}_{\mu}$. Tanto a definição da classe $\mathcal{F}$, como da integração $\int$ passam pela definição de uma classe especial de conjuntos chamados de conjuntos mensuráveis, denotados $\mathcal{A}$, e regras de medida $\mu: \mathcal{A} \rightarrow \mathbb{R}^{+}$. De modo que as funções mensuráveis sejam $f=\sum_{n=0}^{\infty} a_{n} \chi_{A_{n}}$ e as integrais $\int f d \mu=\sum_{n=0}^{\infty} a_{n} \mu\left(A_{n}\right)$. Para tanto, $\mathcal{A}$ e $\mu$ precisam ter propriedades especiais. Ocorre $\mathcal{S}$ e $\mu: \mathcal{S} \rightarrow \mathbb{R}^{+}$são a mínima informação que precisa ser especificada para definir unicamente um espaço de medidas $(\Omega, \mathcal{A}, \mu)$. O processo que passa de $(\Omega, S, \mu)$ para $(\Omega, \mathcal{A}, \mu)$ é chamado de extensão de Lebesgue. Assim sendo, voltemos ao problema original.

Dado um operador autoadjunto $A$ no espaço de Hilbert separável $\mathcal{H}$ (que admite base normalizável contável, como discutido na subseção 4.4.2), existe uma transformação unitária $U$ tal que $\mathcal{H}$ é posto na forma $\sum_{n}{ }^{\oplus} L^{2}\left(\mathbb{R}, \mu_{n}\right)$, a soma direta enumerável (footnote 3 da subseção 5.2 .1 ) de espaços de Hilbert $L^{2}\left(\mathbb{R}, \mu_{n}\right) 3^{3}$ onde, em cada $L^{2}\left(\mathbb{R}, \mu_{n}\right), A$ se representa como operador multiplicativo $\psi(\lambda) \rightarrow \lambda \psi(\lambda)$.

\footnotetext{
${ }^{2} S-S_{0}=S \cap S_{0}^{c}, S_{0}^{c}$ é o complementar de $S_{0}$ e o somatório de conjuntos representa a união de conjuntos disjuntos

${ }^{3} \mathrm{O}$ espaço de Hilbert das funções $f: \mathbb{R} \rightarrow \mathbb{C}$ de módulo quadrado Lebesgue integrável com respeito a medida de integração $\mu_{n}$, ou seja,o produto interno se escreve $(\phi(\lambda), \psi(\lambda))=\int d \mu_{n} \phi^{*}(\lambda) \psi(\lambda)$
} 
De fato, utilizamos a projeção espectral prevista pelo teorema de Von Neumann, $E(\lambda)$. Seja $\mathcal{H}_{1}$ um subespaço de $\mathcal{H}$ de vetores da forma $\Psi_{f}=\int f(\lambda) d E(\lambda) \Psi_{1}$, sendo $\Psi_{1} \in \mathcal{H}$ um vetor arbitrário. A norma desses vetores é dada pela a integral de Stieltjes: $\int f^{*}(\lambda) f(\lambda) d\left(\Psi_{1}, E(\lambda) \Psi_{1}\right)$. Assim sendo, podemos mapear isometricamente $\mathcal{H}_{1}$ no espaço de Hilbert $L^{2}\left(\mathbb{R}, \mu_{1}\right)$, em que $\mu_{1}$ é a medida associada à integral de Stieltjes: $\int d \mu_{1}=\int d\left(\Psi_{1}, E(\lambda) \Psi_{1}\right) . \mathcal{H}_{1}$ pode ou não ser o espaço de Hilbert total. Se for, o teorema está provado, se não, $\mathcal{H}_{1}$ define um subespaço fechado $\mathcal{M}_{1} \subset \mathcal{H}$. Escolhemos então $\Psi_{2}$ no complemento ortogonal de $\mathcal{M}_{1}$, e repetimos o processo construindo o espaço $\mathcal{M}_{2}$. Como o espaço de Hilbert é separável, esse processo pode, no máximo, gerar uma coleção enumerável de subespaços ortogonais $M_{n}, n=1,2, \cdots$, tal que $\sum_{n}{ }^{\oplus} \mathcal{M}_{n}=\mathcal{H}$, estabelecendo uma correspondência unitária $U: \mathcal{H}=\sum_{n}{ }^{\oplus} \mathcal{M}_{n} \rightarrow \sum_{n}{ }^{\oplus} L^{2}\left(\mathbb{R}, \mu_{n}\right)$ :

$$
\left(\int f_{1}(\lambda) d E(\lambda) \Psi_{1}, \int f_{2}(\lambda) d E(\lambda) \Psi_{2}, \cdots\right) \rightarrow\left(f_{1}(\lambda), f_{2}(\lambda), \cdots\right)
$$

Pelo teorema de Von Neumann, $A=\int \lambda d E(\lambda)$, o que implica que, dado $\Psi=\left(f_{1}(\lambda), f_{2}(\lambda), \cdots\right)$,

$$
U A U^{-1} \Psi=\left(\lambda f_{1}(\lambda), \lambda f_{2}(\lambda), \cdots\right), \Psi \in \sum_{n}^{\oplus} L^{2}\left(\mathbb{R}, \mu_{n}\right)
$$

Todos os espaços de Hilbert $L^{2}\left(\mathbb{R}, \mu_{n}\right)$ possuem a mesma $\sigma$-álgebra $\mathcal{A}$ (obtida do mesmo semi-anel por extensão de Lebesgue), os chamados conjuntos Borel, mas medidas $\mu_{n}$ diferentes. Podemos construir uma nova medida a partir dessas:

$$
\mu(\Delta)=\sum_{n=1}^{\infty} \frac{1}{2^{n}} \mu_{n}(\Delta), \Delta \in \mathcal{A}
$$

esse medida é finita para todo $\Delta \in \mathcal{A}$, pois $\mu_{j}(\Delta)=\int \chi_{\Delta} d\left(\Psi_{i}, E(\lambda) \Psi_{i}\right) \leq\left(\Psi_{i}, \Psi_{i}\right)=1, \chi_{\Delta}$ a função característica do conjunto $\Delta$. Esta medida satisfaz ainda $\mu(\Delta)=0 \Leftrightarrow \mu_{n}(\Delta)=0$ para todo $n$. O teorema Radon-Nikodym (já utilizado em 2.8) afirma que podemos escrever cada uma dessas medidas como:

$$
\mu_{n}(\Delta)=\int \phi_{n}(\lambda) d \mu(\lambda), \quad \phi_{n}(\lambda) \geq 0 \text { uma função } \mu \text { mensurável }
$$

O que nos permite definir uma nova correspondência isométrica $U_{2}: \sum_{n}{ }^{\oplus} L^{2}\left(\mathbb{R}, \mu_{n}\right) \rightarrow$ $\sum_{n}{ }^{\oplus} L^{2}(\mathbb{R}, \mu): f_{n} \rightarrow f_{n} \sqrt{\phi_{n}}=\psi_{n}$. $U_{2}$, contudo, não é sobrejetivo, mapeia $\sum_{n}{ }^{\oplus} L^{2}\left(\mathbb{R}, \mu_{n}\right)$ no subespaço $\sum_{n}{ }^{\oplus} L^{2}\left(A_{n}, \mu\right)$, onde $A_{n} \subseteq \mathbb{R}$ é tal que $\phi_{n}(\lambda)>0$. Assim sendo, a correspondência:

$$
\sum_{n}^{\oplus} L^{2}\left(\mathbb{R}, \mu_{n}\right) \rightarrow \sum_{n}^{\oplus} L^{2}\left(A_{n}, \mu\right)
$$


é unitária.

Seja $N(\lambda)$ o número de conjuntos $A_{n}$ tal que $\lambda \in A_{n}$. Os valores de $N(\lambda)$ são $0,1,2, \cdots \infty$. Sejam $n_{1}(\lambda)<n_{2}(\lambda)<\cdots<n_{N}(\lambda)$ os valores de $n$ tal que $\lambda \in A_{n}$. Escolha para cada $\lambda$ um espaço de Hilbert $\mathcal{H}(\lambda) N(\lambda)$-dimensional, e escolha em $\mathcal{H}(\lambda)$ uma base ortonormal $e_{1}(\lambda), e_{2}(\lambda), \cdots e_{N}(\lambda)$. Defina então, para cada $\lambda$, a correspondência: $\left(\psi_{1}(\lambda), \psi_{2}(\lambda), \cdots\right) \rightarrow \psi_{n_{1}(\lambda)} e_{1}(\lambda)+\psi_{n_{2}(\lambda)} e_{2}(\lambda)+\cdots+\psi_{n_{N}} e_{N}(\lambda)$. Acabamos de definir o espaço de Hilbert:

$$
\mathfrak{h}=\int^{\oplus} \mathcal{H}(\lambda) d \mu(\lambda)
$$

cujos vetores se escrevem

$$
\Psi=\int^{\oplus} \Psi(\lambda) d \mu(\lambda), \quad \Psi(\lambda) \in \mathcal{H}(\lambda)
$$

e cujo produto interno é:

$$
(\Phi, \Psi)=\int\langle\Phi(\lambda), \Psi(\lambda)\rangle_{\lambda} d \mu(\lambda)
$$

$\Psi(\lambda), \Phi(\lambda) \in \mathcal{H}(\lambda)$ e $\langle *, *\rangle_{\lambda}$ o produto interno de $\mathcal{H}(\lambda)$. A transformação $\sum_{n}{ }^{\oplus} L^{2}\left(A_{n}, \mu\right) \rightarrow$ $\int^{\oplus} \mathcal{H}(\lambda) d \mu(\lambda)$ é unitária pela $\sigma$-aditividade da integral de Lebesgue:

$$
\sum_{n} \int\left|\psi_{n}(\lambda)\right|^{2} d \mu=\int \sum_{n}\left|\psi_{n}(\lambda)\right|^{2} d \mu .
$$

Compondo as transformações:

$$
\mathcal{H} \rightarrow \sum_{n}^{\oplus} L^{2}\left(\mathbb{R}, \mu_{n}\right) \rightarrow \sum_{n}^{\oplus} L^{2}\left(A_{n}, \mu\right) \rightarrow \int^{\oplus} \mathcal{H}(\lambda) d \mu(\lambda),
$$

definimos também uma transformação unitária $U_{\mathcal{D}}: \mathcal{H} \rightarrow \int^{\oplus} \mathcal{H}(\lambda) d \mu(\lambda)$, tal que:

$$
U_{\mathcal{D}} A U_{\mathcal{D}}^{-1} \int^{\oplus} \Psi(\lambda) d \mu(\lambda)=\int^{\oplus} \lambda \Psi(\lambda) d \mu(\lambda)
$$

O domínio do operador $A$ passa a ser o o conjunto dos $\Psi$ tais que:

$$
\int|\lambda|^{2}\|\Psi(\lambda)\|^{2} d \mu(\lambda)<\infty
$$

8.4.1.1 A decomposição integral direta da representação de uma álgebra no espaço de Hilbert

Dado um conjunto de operadores autoadjuntos $C_{1}, C_{2}, \cdots$ com um domínio invariante comum $\mathcal{D} \subseteq \mathcal{H}$ (essencialmente autoadjunto) em que os mesmos comutam, decorre que as 
projeções espectrais $E_{i}\left(\lambda_{i}\right)$ comutam e podemos repetir o mesmo raciocínio acima 4 que nos leva a um resultado análogo: Existe uma transformação unitária

$$
U_{\mathcal{D}}: \mathcal{H} \rightarrow \mathfrak{h}=\int^{\oplus} \mathcal{H}\left(\lambda_{1}, \lambda_{2}, \cdots\right) d \mu\left(\lambda_{1}, \lambda_{2}, \cdots\right)
$$

tal que

$$
U_{\mathcal{D}} A_{i} U_{\mathcal{D}}^{-1} \int^{\oplus} \Psi\left(\lambda_{1}, \lambda_{2}, \cdots\right) d \mu\left(\lambda_{1}, \lambda_{2}, \cdots\right)=\int^{\oplus} \lambda_{i} \Psi\left(\lambda_{1}, \lambda_{2}, \cdots\right) d \mu\left(\lambda_{1}, \lambda_{2}, \cdots\right)
$$

O subespaço $\mathcal{D}$ é definido pelo requerimento:

$$
\int^{\oplus}\left|\lambda_{i}\right|^{2}|| \Psi\left(\lambda_{1}, \lambda_{2}, \cdots\right) \|^{2} d \mu\left(\lambda_{1}, \lambda_{2}, \cdots\right)<\infty
$$

Suponha que em cada espaço de Hilbert $\mathcal{H}(\lambda)$ esteja definido um operador $X(\lambda)$ num subespaço $\mathcal{D}(\lambda) \subseteq \mathcal{H}(\lambda)$, podemos definir um novo operador $X$ em $\mathfrak{h}$ da forma:

$$
X \Psi=\int^{\oplus} X(\lambda) \Psi(\lambda) d \mu(\lambda)
$$

definido no domínio $\mathcal{D}=\left\{\Psi(\lambda) \in \mathcal{D}(\lambda) ; \int d \mu(\lambda)\langle X(\lambda) \Psi(\lambda), X(\lambda) \Psi(\lambda)\rangle_{\lambda}<\infty\right\}$. Sendo $X(\lambda)$ autoadjunto no domínio $\mathcal{D}(\lambda), X$ é autoadjunto em $\mathcal{D}$ e podemos definir qualquer função de $X$ (num domínio adequado):

$$
f(X) \Psi=\int^{\oplus} f(X(\lambda)) \Psi(\lambda) d \mu(\lambda)
$$

o que pode ser justificado observando-se que

$$
E(t)=\int^{\oplus} E_{\lambda}(t) d \mu(\lambda)
$$

fornece uma decomposição espectral do operador $X$ (Teorema 1) quando $E_{\lambda}(t)$ é uma decomposição espectral do operador $X(\lambda)$ em $\mathcal{H}(\lambda)$. Basta observar que a projeção $P([a, b))=\int \chi_{[a, b)} d E(t)$ satisfaz:

$$
a P([a, b)) \Psi \leq X P([a, b)) \Psi \leq b P([a, b)) \Psi
$$

essa desigualdade é interpretada segundo a propriedade 1 do referido teorema. Além disso, essa desigualdade é essencialmente a propriedade que permite escrever o operador $A$ como

\footnotetext{
${ }^{4}$ Começando por expressões do tipo $\int f\left(\lambda_{1}, \lambda_{2}, \cdots\right) d E\left(\lambda_{1}\right) E\left(\lambda_{2}\right) \cdots$ para definir a primeira transformação unitária
} 
a integral 5.4. Mas, ainda segundo o teorema, só existe uma decomposição espectral, de modo que precisa ser esta.

Suponha então uma representação $\pi$ de uma $C^{*}$-álgebra ou *álgebra $\mathcal{A}$ no espaço de Hilbert $\mathcal{H}$ que tem como conjunto de operadores de Casimir autoadjuntos $C_{i}, i=$ $1,2, \cdots$. Podemos obter uma representação em $\mathfrak{h}$ unitariamente equivalente a $\pi$ fazendo $\pi \rightarrow U_{\mathcal{D}} \pi U_{\mathcal{D}}^{-1}$. Ocorre que em cada um dos espaços de Hilbert $\mathcal{H}\left(\lambda_{1}, \lambda_{2}, \cdots\right)$ os operadores de Casimir são múltiplos da identidade, decorre então do Lema de Shur (Lema 1) que em cada um dos $\mathcal{H}\left(\lambda_{1}, \lambda_{2}, \cdots\right)$ atua uma representação irredutível de $\mathcal{A}$. Na verdade, atua uma representação irredutível de $\mathcal{A}$ se não houver nenhum outro operador $A$ autoadjunto de domínio invariante comum que ainda comute com a representação, ainda que não pertença a ela. Por exemplo, suponha $\pi: \mathcal{A}^{\prime} \rightarrow \mathcal{B}(\mathcal{H})$ uma representação da $C^{*}$-álgebra $\mathcal{A}^{\prime}$ com um Casimir $C^{\prime}$. Outra representação é $\pi^{\prime}=\pi \oplus \pi$, não existe nenhum outro elemento na imagem de $\pi^{\prime}$ (i.e. da representação) que comute com $\pi^{\prime}\left(\mathcal{A}^{\prime}\right)$, a não ser $\pi^{\prime}\left(C^{\prime}\right)$. Mas $C^{\prime \prime}=$ $\left(a C^{\prime}\right) \oplus\left(b C^{\prime}\right), a, b \in \mathbb{R}, a \neq b$, comuta. A conclusão correta é, por tanto, que $\mathcal{H}\left(\lambda_{1}, \lambda_{2}, \cdots\right)$ se decompõe em $\nu\left(\lambda_{1}, \lambda_{2}, \cdots\right)$ (finito ou infinito) cópias da mesma representação irredutível associada ao mesmo conjunto de autovalores de Casimir. Temos então uma prescrição para decompor uma representação qualquer de uma álgebra em representações irredutíveis.

Pelo argumentado, dada uma decomposição integral direta da representação $\pi$ de $\mathfrak{p}_{\mathcal{N}}$ (com respeito à totalidade dos operadores de Casimir), $\pi\left(X_{i}\right)$ se escreve como:

$$
\pi\left(X_{i}\right) \int^{\oplus} \Psi(\lambda) d \mu(\lambda)=\int^{\oplus} \pi_{\lambda}\left(X_{i}\right) \Psi(\lambda) d \mu(\lambda),
$$

em que $\pi_{\lambda}$ é uma representação irredutível. Segundo 8.22 , esta induz uma representação do grupo $\mathcal{P}_{\mathcal{N}}$ que realiza a mesma álgebra de geradores $\mathfrak{p}_{\mathcal{N}}$ :

$$
e^{-i \pi\left(X_{i}\right) \theta_{i}} \int^{\oplus} \Psi(\lambda) d \mu(\lambda)=\int^{\oplus} e^{-i \pi_{\lambda}\left(X_{i}\right) \theta_{i}} \Psi(\lambda) d \mu(\lambda),
$$

que se escreve:

$$
U=\int^{\oplus} \mathcal{U}_{\lambda}^{\mathcal{P} \mathcal{N}} d \mu(\lambda)
$$

8.4.2 A não existência de um grupo de Lie que realiza a inflação não comutativa

Vamos provar um importante resultado original desta tese: Não existe grupo de Lie que realize a inflação não comutativa e a para qualquer escolha inflacionária de $\mathcal{P}_{\mathcal{N}}$, a representação 7.71 não realiza a mesma álgebra 
$\mathfrak{p}_{\mathcal{N}}$. Ou seja, se a mesma faz parte da formulação da física, não existe uma noção única de relação de dispersão na teoria, partículas podem ser flagradas obedecendo toda uma variedade inequivalente de relações entre energia e momento.

Para tanto, façamos referência a uma condição que emergiu da análise da subseção 8.2 , mas precisamente do Teorema 6 que associa a limitação de momento dos fótons à condição de número mínimo de e-foldings que precisa ser satisfeita pela inflação.

Considere as restrições sobre o conjunto $V_{\mathcal{P}_{\mathcal{N}}}^{+}$(definido na propriedade 7 que foi referenciada na seção 7.3 que emergem de assumir que a representação $C(E, P)=\lambda_{F} \in M_{\mathcal{P}_{\mathcal{N}}}$ descreve a radiação. Suponha que $\mathcal{C}_{\lambda_{F}}$ contenha o ponto $(E, P)=(0,0)$ e, para qualquer outro $\lambda \in V_{\mathcal{P}_{\mathcal{N}}}^{+}, \mathcal{C}_{\lambda}$ contenha o ponto $(E, P)=\left(E_{\lambda}, 0\right)$. Uma vez que $\mathcal{C}_{\lambda}$ é rotacionalmente invariante por hipótese, podemos considerar apenas o gráfico $\|P\|=P_{\lambda}(E)$. Ocorre que $P_{\lambda}(E) \geq 0$ e não existe $E$ tal que $P_{\lambda_{1}}(E)=P_{\lambda_{2}}(E)$ para $\lambda_{1} \neq \lambda_{2}$. Decorre que $P_{\lambda}\left(E_{\lambda}\right)<P_{\lambda_{F}}\left(E_{\lambda}\right)$, o que implica que $P_{\lambda}(E)<P_{\lambda_{F}}(E)$ para todo $E$, pois se existisse $E$ tal que $P_{\lambda}(E)>P_{\lambda_{F}}(E)$, da continuidade de $P_{\lambda}(E)$ que segue da analiticidade do Casimir $C(E, P)$, precisaria existir $E$ tal que $P_{\lambda}(E)=P_{\lambda_{F}}(E)$, mas tal ponto não pode existir. Decorre que se toda partícula tiver um estado de momento zero, uma limitação do momento para a representação dos fótons implica numa limitação do momento de todas as representações irredutíveis.

Vamos determinar agora o espectro do operador $P^{\mu}$ da representação 8.27 de $\mathfrak{p}_{\mathcal{N}}$ :

$$
\mathfrak{h}=\int^{\oplus} \mathcal{H}(\lambda, \sigma) d \mu(\lambda, \sigma),
$$

onde $\lambda$ é o autovalor do Casimir da relação de dispersão e $\sigma$ representa todos os demais. A medida $\mu$ pode ser escrita como o produto das medidas em $\lambda$, denotada $\nu_{1}$, e em $\sigma$, denotada $\nu_{2}$ (Os conjuntos mensuráveis são os produtos cartesianos $A \times B$ em que $A$ é um conjunto mensurável de $\lambda$ e $B$ um conjunto mensurável de $\sigma$ e a medida se escreve como o produto das medidas $\left.\nu_{1}(A) \nu_{2}(B)\right)$.

Podemos escrever $P^{\mu}$ como operador multiplicativo se aplicarmos uma transformação unitária em $\mathfrak{h}$ que expressa cada $\mathcal{H}(\lambda, \sigma)$ como o espaço de Hilbert das funções definidas no local dos pontos $(E, \vec{P}) \in \mathbb{R}^{4}$ que satisfazem $C(E, P)=\lambda, \mathcal{C}_{\lambda}$, e com uma medida $\nu_{0}$. 
Combinado as medidas em $\mathcal{C}_{\lambda}$ e em $\lambda$, definimos uma medida $\mu^{\prime}$ em $\mathbb{R}^{4}$ e escrevemos:

$$
\int^{\oplus} \mathcal{H}\left(p^{\mu}, \sigma\right) d \mu^{\prime}\left(p^{\mu}\right) d \nu_{2}(\sigma)
$$

o suporte da medida $\nu_{0}$ (a união de todos os conjuntos de medida não nula) é o conjunto $\operatorname{dos} \mathcal{C}_{\lambda}$ tal que $\lambda$ pertence à decomposição.

Agora, suponha que na decomposição apareçam representações $\mathcal{U}_{\lambda}^{\mathcal{P}_{\mathcal{N}}}$ com autovalores $\lambda_{0} \notin M_{\mathcal{P}_{\mathcal{N}}}$, para algum conjunto $B$ dos $\lambda$ de medida não nula. Suponha uma função mensurável $f(\lambda)$ tal que $\int d \mu(\lambda)|f(\lambda)|^{2}<\infty$. Se $B$ tiver medida finita, então esta função pode ser escolhida a função característica de $B, \chi_{B}$. Podemos então escolher um vetor normalizado $\psi_{\lambda}$ de energia negativa $E_{\lambda}$ em cada $\mathcal{H}(\lambda)$ com $\lambda \in B$ e o vetor nulo nos demais valores de $\lambda$. O estado $\int^{\oplus} d \mu(\lambda) f(\lambda) \psi_{\lambda}$ pertence ao espaço de Hilbert $\mathfrak{h}$ e tem energia negativa. Decorre que em toda decomposição integral direta de energia positivodefinida de $\mathfrak{p}_{\mathcal{N}}$, quando colocada na representação de momento, 8.29, para todo $\lambda, p^{\mu}$ é um operador multiplicativo e pertence ao conjunto $V^{+}$.

Suponha o operador $P^{\mu}$ definido em $\mathfrak{h}$ pelo operador $P_{\lambda}^{\mu}$ em cada $\mathcal{H}(\lambda)$, tal que, para todo $\lambda, P_{\lambda}^{\mu}\left|\Psi_{\lambda}\right\rangle=p^{\mu}\left|\Psi_{\lambda}\right\rangle, p^{\mu} \in V^{+} \subseteq \mathbb{R}^{4}$, então, para todo $|\Psi\rangle$ normalizado, $\left\langle\Psi\left|P^{\mu}\right| \Psi\right\rangle \in$ $\bar{V}^{+}{ }_{c}$, o fechamento linear convexo, conjunto de todos pontos da forma $\alpha p_{1}^{\mu}+(1-\alpha) p_{2}^{\mu}$, $\alpha \in[0,1]$ e $p_{1}^{\mu}, p_{2}^{\mu} \in V^{+} . \overline{V^{+}}{ }_{c}$ é obtido unindo a $V^{+}$todo segmento de reta que liga dois pontos quaisquer de $V^{+}$. De fato, podemos particionar o conjunto $V^{+}$em retângulos

$$
R_{i j k l}=\left[p_{i}^{0}, p_{i+1}^{0}\right) \times\left[p_{j}^{1}, p_{j+1}^{1}\right) \times\left[p_{k}^{2}, p_{k+1}^{2}\right) \times\left[p_{l}^{3}, p_{l+1}^{3}\right),
$$

obtidos de partições $p_{i}^{\mu}, i=1,2, \cdots N, \mu=0,1,2,3$, tais que $\bigcup_{n} R_{n} \supset V \cdot\left\langle\Psi\left|P^{\mu}\right| \Psi\right\rangle=$ $\int d \mu p^{\mu}\|\psi(\lambda)\|^{2}$ que implica na desigualdade:

$$
\sum_{n} \inf _{R_{n} \cap V}\left(p^{\mu}\right) \int_{R_{n}}\|\psi(\lambda)\|^{2} d \mu \leq\left\langle\Psi\left|P^{\mu}\right| \Psi\right\rangle \leq \sum_{n} \sup _{R_{n} \cap V}\left(p^{\mu}\right) \int_{R_{n}}\|\psi(\lambda)\|^{2} d \mu,
$$

mas $\|\psi(\lambda)\|^{2}$ é positivo definido e $\int d \mu\|\psi(\lambda)\|^{2}=1$, de modo que tanto o lado esquerdo como o direito da desigualdade se escrevem como: $\sum_{n=0}^{\infty} p_{n}^{\mu} c_{n}, \sum_{n} c_{n}=1$ e $p_{n}^{\mu} \in V$. Podemos reescrever essa soma como:

$$
\sum_{n=0}^{\infty} p_{n}^{\mu} c_{n}=\lim _{m \rightarrow \infty} \frac{\sum_{n=0}^{m} p_{n}^{\mu} c_{n}}{\sum_{n=0}^{m} c_{n}}=\lim _{m \rightarrow \infty} \sum_{n=0}^{m} p_{n}^{\mu} d_{n}^{(m)}, \quad \sum_{n=0}^{m} d_{n}^{(m)}=1
$$

podemos escrever:

$$
\sum_{n=0}^{1} p_{n}^{\mu} d_{n}^{(1)}=\alpha_{1} p_{1}^{\mu}+\left(1-\alpha_{1}\right) p_{2}^{\mu}
$$


onde $\alpha_{1}=d_{1}^{(1)}$

$$
\sum_{n=0}^{3} p_{n}^{\mu} d_{n}^{(3)}=\beta_{1}\left(\alpha_{1} p_{1}^{\mu}+\left(1-\alpha_{1}\right) p_{2}^{\mu}\right)+\left(1-\beta_{1}\right)\left(\alpha_{2} p_{3}^{\mu}+\left(1-\alpha_{2}\right) p_{4}^{\mu}\right),
$$

onde $\alpha_{1}=\frac{d_{1}^{(3)}}{d_{1}^{(3)}+d_{2}^{(3)}}$ e $\beta_{1}=\frac{d_{1}^{(3)}+d_{2}^{(3)}}{d_{1}^{(3)}+d_{2}^{(3)}+d_{3}^{(3)}+d_{4}^{(3)}}$; Podemos repetir o mesmo procedimento para $\sum_{n=0}^{2^{m}-1} p_{n}^{\mu} d_{n}^{(m)}$, definido $m$ combinações lineares convexas. Fazer o tamanho dos retângulos tender a zero conclui a demonstração.

Assim sendo, se para todo $p^{\mu} \in V^{+},\|\vec{p}\|<p_{\max }$, decorre que em toda representação de $\mathfrak{p}_{\mathcal{N}}$ com energia positiva definida o momento é limitado:

$$
\left\langle\Psi\left|\vec{P}^{2}\right| \Psi\right\rangle \leq p_{\max }
$$

Mas os geradores associados à representação 7.71 são da forma:

$$
X_{F}=\sum_{N=1}^{\infty} \oplus(X \otimes I \cdots \otimes I+I \otimes X \otimes \cdots \otimes I+\cdots+I \otimes I \cdots \otimes X)_{N}
$$

Em particular, a energia é positiva definida e o momento é ilimitado (pode ser qualquer múltiplo inteiro de energia de uma representação irredutível). Logo, os geradores de 7.71 não realizam $\mathfrak{p}_{\mathcal{N}}$ e, além disso, nenhuma realização de $\mathcal{P}_{\mathcal{N}}$ da forma 7.71 realiza $\mathfrak{p}_{\mathcal{N}}$. Como toda realização de um grupo de Lie realiza a mesma álgebra de Lie e 7.71 é uma representação do grupo $\mathcal{P}_{\mathcal{N}}$, nenhum grupo de Lie pode realizar a inflação não comutativa.

\subsubsection{A prescrição $\pi_{\mathcal{A}}$}

O argumento da subseção anterior sugere que a representação 7.71, que leva às equações da inflação não comutativa, leva a problemas conceituais. Propomos então uma nova prescrição para formular a física não relativística que evita esse problema. Vamos também generalizar o alcance da prescrição para toda a teoria de campos relativística, não apenas para a teoria livre. Utilizaremos a mesma análise já feita em 7.4.2, mas tomaremos um caminho diferente para definir a representação de $\mathcal{P}_{\mathcal{N}}$ que descreve a física. Este é o principal resultado desta tese.

A primeira questão é descobrir qual representação do grupo de Poincaré descreve a matéria numa teoria quântica relativística. Esta é justamente a representação que aparece 
em 6.43, quando esta está definida num espaço de Hilbert de métrica positiva definida 5 . A qual pode ser obtida pelo teorema de reconstrução de Wightman. O teorema de reconstrução de Wightman é na verdade uma aplicação da construção GNS já discutida na subseção 7.4.2, a ideia básica é obter uma representação para os campos quânticos a partir do valor esperado no vácuo do produto de $N$ campos, $\left\langle 0\left|\phi\left(x_{1}\right) \cdots \phi\left(x_{N}\right)\right| 0\right\rangle$, que é o objeto que se calcula (perturbativamente) na prática (ou outra classe de funções similar, pois existem muitas hierarquias de funções desse tipo, como o produto ordenado no tempo e a continuação analítica para o tempo imaginário dessas funções, conhecidas como funções de Green Euclidianas). Como argumentado muitas vezes, $\phi(x)$ não é um operador no sentido convencional (veja o comentário no footnote 24 do capítulo 5]. Então o que se realiza pela construção $G N S$ são expressões do tipo $\int d^{4} x \phi(x) f(x)$, onde $f(x)$ é uma função teste de valor complexo. Mas isso é uma correspondência entre funções teste $f(x)$ e operadores. Podemos então pensar que as funções teste $f(x)$ são os próprios operadores e construir uma álgebra para elas. Podemos associar sequências finitas de funções teste em $N$ variáveis.

$$
f=\left(f_{0}, f_{1}\left(x_{1}\right), f_{2}\left(x_{1}, x_{2}\right), f_{3}\left(x_{1}, x_{2}, x_{3}\right), \cdots f_{n}\left(x_{1}, x_{2}, x_{3}, \cdots x_{n}\right), \cdots\right)
$$

a expressões do tipo

$$
P(\phi, f)=f_{0}+\int d^{4} x f\left(x_{1}\right) \phi\left(x_{1}\right)+\iint d^{4} x d^{4} x \phi\left(x_{1}\right) \phi\left(x_{2}\right) f\left(x_{1}, x_{2}\right)+\cdots
$$

definimos a involução em $f$ pela conjugação complexa dos termos, combinações lineares a coeficientes complexos são definidas por aquelas termo a termo. Definimos o produto das sequências 8.37 segundo o produto 8.38 :

$$
f \times g=\left(f_{0} g_{0}, f_{0} g_{1}\left(x_{1}\right)+g_{0} f_{1}\left(x_{1}\right), f_{0} g_{2}\left(x_{1}, x_{2}\right)+f_{1}\left(x_{1}\right) g_{1}\left(x_{2}\right)+g_{1}\left(x_{1}\right) f_{1}\left(x_{2}\right)+f_{2}\left(x_{1}, x_{2}\right) g_{0}, \cdots\right)
$$

Para realizar essa *-álgebra via GNS, necessitamos apenas de um funcional linear positivo definido, i.e. $\omega\left(f \times f^{*}\right) \geq 0$. Mas esses funncionais são dados pelos funcionais de Wightman:

$$
\omega(f)=\langle 0 \mid 0\rangle f_{0}+\int d^{4} x\left\langle 0\left|\phi\left(x_{1}\right)\right| 0\right\rangle f\left(x_{1}\right)+\int d^{4} x_{1} d^{4} x_{2}\left\langle 0\left|\phi\left(x_{1}\right) \phi\left(x_{2}\right)\right| 0\right\rangle f\left(x_{1}, x_{2}\right)+\cdots .
$$

\footnotetext{
${ }^{5}$ Nem todos os campos podem satisfazer a covariância relativística num espaço de Hilbert de métrica positiva definida, isto é, $(\Psi, \Psi) \geq 0$ para todo $\Psi$. Dependendo da representação $V_{i j}$ de $S L(2, \mathbb{C})$, essa possibilidade é excluída por um argumento similar ao que se utilizou para demonstrar 6.55
} 
Observamos que $\left\langle 0\left|\phi\left(x_{1}\right) \cdots \phi\left(x_{N}\right)\right| 0\right\rangle$ são distribuições. A representação do grupo de Poincaré associada é dada por;

$$
U(\Lambda, a)[f]=\left[\left(f_{0}, f_{1}\left(\Lambda x_{1}+a\right), f_{2}\left(\Lambda x_{1}+a, \Lambda x_{2}+a\right), \cdots\right)\right]
$$

em que $[f]$ é a classe de equivalência de $f$, os vetores da construção GNS.

E quando o campo relativisticamente covariante não puder ser definido num espaço de Hilbert de métrica positiva definida? Esse caso é o mais importante, visto que é o caso do campo eletromagnético descrito pelo potencial vetor $A^{\mu}$, campo este que se supõe dirigir a inflação não comutativa. Nesse caso, ainda temos uma transformação isométrica definida por 8.41, mas esse transformação deixa invariante o subespaço de métrica positiva definida (Os vetores $|\Psi\rangle$ não nulos tais que $\langle\Psi \mid \Psi\rangle>0$ ). A restrição de 8.41 a este subespaço é a transformação unitária que procuramos.

Decompomos então esta representação na forma integral direta:

$$
\mathcal{U}^{\mathcal{P}}=\mathcal{U}_{0} \oplus \int^{\oplus} d \mu(\lambda) \sum_{\sigma}^{\oplus} \nu(\lambda, \sigma) \mathcal{U}_{\lambda, \sigma}^{\mathcal{P}},
$$

que atua no espaço de Hilbert:

$$
\mathcal{H}=\left(c \Psi_{0}\right) \oplus \int^{\oplus} d \mu(\lambda) \sum_{\sigma}^{\oplus} \nu(\lambda, \sigma) \mathcal{H}_{\lambda, \sigma}^{\mathcal{P}},
$$

onde $\mathcal{U}_{\lambda, \sigma}^{\mathcal{P}}$ define uma representação irredutível do grupo de Poincaré rotulado pelo autovalor $\lambda=m^{2}$ do Casimir $P^{2}$ e o índice discreto $\sigma$ (spin ou helicidade) associado ao autovalor discreto dos demais operadores de Casimir (essas representações foram discutidas no capítulo 5). $\mathcal{U}_{0}$ é a representação trivial do vácuo, que ocorre por postulado sobre a existência de um estado invariante pela ação do grupo de Poincaré, o vácuo $\Psi_{0}$. $\nu(\sigma, \lambda)$ é a degenerescência da representação (i.e. o número de vezes em que a mesma representação aparece na decomposição) que pode ser finita ou infinita. $c \Psi_{0}$ representa o subespaço unidimensional gerado pelo vácuo (as demais representações não triviais atuam no complemento ortogonal deste subespaço).

Postulamos então a alternativa a 7.71, Na decomposição integral direta de 8.41, 8.42, a seguinte substituição define a deformação não relativística da teoria::

$$
\mathcal{U}_{\lambda, \sigma}^{\mathcal{P}} \rightarrow \mathcal{U}_{\lambda^{\prime}}^{\mathcal{N} \mathcal{P}}
$$


onde $\mathcal{U}_{\lambda^{\prime}}^{\mathcal{N} \mathcal{P}}$ é a representação do grupo $\mathcal{P}_{\mathcal{N}}$ associado à representação irredutível da Hopf álgebra $\mathfrak{p}_{\mathcal{N}}$ (segundo discussão em 7.4.2). A questão deixada em aberto na ocasião é qual o critério utilizado para escolher a representação de Hopf álgebra que substitui cada representação irredutível de Poincaré. O critério que postulamos é que cada $\mathcal{U}_{\lambda, \sigma}^{\mathcal{P}}$ recupere, no limite de baixa energia e momento, a correspondente representação de Poincaré. Sejamos mais precisos: a correspondência $\lambda^{\prime}(\lambda, \sigma)$ é tal que, dada a representação $\mathcal{U}_{\lambda=m^{2}, \sigma}^{\mathcal{P}}$ no espaço de Hilbert $\mathcal{H}_{\left(m^{2}, \sigma\right)}$, representação esta escolhida tal que $P^{\mu}$ é diagonal, 7.24, existe uma transformação unitária $\pi: \mathcal{H}_{\left(m^{2}, \sigma\right)} \rightarrow \mathcal{H}_{\lambda^{\prime}}$, onde $\mathcal{H}_{\lambda^{\prime}}$ é o espaço de Hilbert onde se realiza $\mathcal{U}_{\lambda^{\prime}}^{\mathcal{N} \mathcal{P}}$, tal que dado $\Psi$ da forma 7.26 :

$$
\left(\pi\left(\Psi_{\lambda, \sigma}\right), \mathcal{U}_{\lambda^{\prime}}^{\mathcal{N} \mathcal{P}} \pi\left(\Phi_{\lambda, \sigma}\right)\right) \rightarrow\left(\Psi_{\lambda, \sigma}, \mathcal{U}_{\lambda, \sigma}^{\mathcal{P}} \Phi_{\lambda, \sigma}\right) \text { quando } E_{\text {max }}, p_{\text {max }}^{j} \rightarrow 0
$$

Implícito na definição é que existe uma regra que faz corresponder cada transformação unitária de $\mathcal{U}_{\lambda^{\prime}}^{\mathcal{N}}$ em cada representação unitária $\mathcal{U}_{\lambda, \sigma}^{\mathcal{P}}$. É então possível provar, pelo $\boldsymbol{t} \boldsymbol{e}$ orema da convergência dominada de Lebesgue ${ }^{6}$, que a representação associada de $\mathfrak{p}_{\mathcal{N}}$ não introduz infinitos adicionais na teoria (evitando por construção problemas com renormalização) e converge no sentido fraco (i.e., no sentido de elementos de matriz: $\left.\left(\pi(\Psi), \mathcal{U}^{\mathcal{N} \mathcal{P}} \pi(\Phi)\right) \rightarrow\left(\Psi, \mathcal{U}^{\mathcal{P}} \Phi\right)\right)$ para a correspondente representação de Poincaré se todos os estados da decomposição integral direta, escritos da forma 7.26, convergem na forma requerida, o que se traduz como o limite de baixa energia e momento dos estados da representação total.

Vamos sumarizar a prescrição exposta:

Prescrição $2\left(\pi_{\mathcal{A}}\right)$. Seja $\mathfrak{p}_{\mathcal{N}}$ uma Hopf álgebra com geradores $X_{i}$ e $\mathcal{P}_{\mathcal{N}}$ o grupo gerado pelos subgrupos de um parâmetro da forma $e^{-i X_{i} \theta_{i}}, t \in \mathbb{R}$. Seja $\pi_{\lambda^{\prime}}: \mathfrak{p}_{\mathcal{N}} \rightarrow O(\mathcal{D})$ (em que $O(\mathcal{D})$ são os operadores essencialmente autoadjuntos no dominio invariante $\mathcal{D}$ ) a representação irredutivel de $\mathfrak{p}_{\mathcal{N}}$ associada com conjunto de autovaleres de Casimir $\lambda^{\prime}=\left(\lambda_{0}, \lambda_{i}\right)$. Seja $\mathcal{U}_{\lambda^{\prime}}^{\mathcal{P}_{\mathcal{N}}}$ a representação de $\mathcal{P}_{\mathcal{N}}$ gerada por elementos da forma $e^{-i \pi_{\lambda^{\prime}}\left(X_{i}\right) t}$. A repre-

6 O teorema de Lebesgue diz que, se $g_{N}(x) \rightarrow g(x)$ para todo $x$ e $\left|g_{N}(x)\right| \leq f(x)$ para alguma função integrável $f(x)$, então $g(x)$ é Lebesgue integrável e $\int g_{N}(x) \rightarrow \int g(x)$. Uma vez que sabemos que para a teoria de campos original existem vetores $\Psi_{\sigma, \lambda}$ em cada $\mathcal{H}_{\lambda, \sigma}$ tais que a integral do produto interno converge, e a transformação unitária satisfaz $\left|\left(\pi\left(\Psi_{\lambda, \sigma}\right), \mathcal{U}_{\lambda^{\prime}, \sigma^{\prime}}^{\mathcal{N} \mathcal{P}} \pi\left(\Phi_{\lambda, \sigma}\right)\right)\right| \leq\left\|\Psi_{\lambda, \sigma}\right\| \cdot\left\|\Phi_{\lambda, \sigma}\right\| \leq$ $\left\|\Phi_{\lambda, \sigma}\right\|^{2}+\left\|\Psi_{\lambda, \sigma}\right\|^{2}$ que é uma função mensurável com integral finita, temos o cumprimento das condições do teorema de Lebesgue. 
sentação de $\mathcal{P}_{\mathcal{N}}$ que deforma a teoria física descrita pelo conjunto de funções de $N$-pontos $\left\langle 0\left|\phi_{i}\left(x_{1}\right) \cdots \phi_{k}\left(x_{N}\right)\right| 0\right\rangle$ (calculada perturbativamente ou exatamente) é obtida da transformação unitária associada reconstrução de Wightman 8.41, restrita ao subespaço físico, posta na forma integral direta

$$
\mathcal{U}^{\mathcal{P}}=\mathcal{U}_{0} \oplus \int^{\oplus} d \mu(\lambda) \sum_{\sigma}^{\oplus} \nu(\lambda, \sigma) \mathcal{U}_{\lambda, \sigma}^{\mathcal{P}},
$$

pela a substituição:

$$
\mathcal{U}_{\lambda} \rightarrow \mathcal{U}_{\lambda^{\prime}}^{\mathcal{P}_{\mathcal{N}}}
$$

para conjunto $\lambda^{\prime}$ satisfazendo a condição 8.44.

\subsection{A existência da estrutura algébrica admissível}

Vamos encerrar esta tese mostrando que existe $\mathfrak{p}_{\mathcal{N}}$ (não único) satisfazendo todas as restrições consideradas na seção 7.3 (não consideramos propriedades coalgébricas aqui, como o coproduto), para qualquer relação de dispersão da forma $C(E, p)=f^{2}(E) p^{2}-E^{2}$, empregada na inflação não comutativa. Outro resultado orginal desta tese.

De fato, considere $X_{i}$ os geradores infinitesimais do grupo de Lorentz homogêneo em $\mathbb{R}^{4}$ de coordenadas $p^{\mu}$. Estes podem ser obtidos pelo procedimento

$$
-\left.i \frac{\partial}{\partial \theta_{i}} f\left(\Lambda_{\nu}^{\mu}\left(0, \cdots, \theta_{i}, \cdots, 0\right) p^{\nu}\right)\right|_{\theta_{i}=0}=X_{i} f\left(p^{\mu}\right)
$$

no subespaço das funções diferenciáveis do espaço de Hilbert $\mathcal{H}$ das funções de $\mathbb{R}^{4}$ com produto interno $(f, g)=\int d^{4} p f^{*} g$. Eles são campos vetoriais contravariantes, ou, equivalentemente, operadores diferenciais de primeira ordem:

$$
X_{i}=X_{i}^{\alpha} \partial_{\alpha}
$$

Estes satisfazem a álgebra de Lie de Lorentz no subespaço (essencialmente autoadjunto) das funções infinitamente diferenciáveis de $\mathcal{H}$ :

$$
\left[X_{i}, X_{j}\right]=C_{i j}^{k} X_{k}
$$

Considere o difeomorfismo $\Phi: p^{\mu} \rightarrow \overline{p^{\mu}}$ dado por $\overline{p^{0}}=p^{0} ; \overline{p^{j}}=p^{j} / f(E)$. Defina agora uma nova álgebra de operadores em $\mathcal{H}$, os operadores multiplicativos $E, P\left(E \psi\left(p^{\mu}\right)=\right.$ 
$p^{0} \psi\left(p^{\mu}\right)$ e $\left.P^{j} \psi\left(p^{\mu}\right)=p^{j} \psi\left(p^{\mu}\right)\right)$ e os operadores diferenciais $\overline{X_{i}}=\Phi_{*} X_{i}$, sendo $\Phi_{*}$ o operador pushfoward $\Phi_{*} X_{i} \triangleright f=X_{i} \triangleright f \circ \Phi$, definido em 2.33. Esta nova álgebra satisfaz $[E, P]=0$, a existência de uma álgebra comutativa associada a energia e momento; $\left[\overline{X_{i}}, \overline{X_{j}}\right]=C_{i j}^{k} \overline{X_{k}}$, em que $C_{i j}^{k}$ são as mesmas constantes de estrutura da álgebra de Lie do grupo de Lorentz 8.50, em particular preserva a álgebra de Lie de $S O(3),\left[M_{i}, M_{j}\right]=i \epsilon_{i j k} M_{k}$ e $\left[H, M_{j}\right]=$ $0 ;\left[\overline{X_{i}}, E\right]=\left(\overline{X_{i}} \triangleright E\right)=F(E, P)$ e $\left[\overline{X_{i}}, p\right]=\left(\overline{X_{i}} \triangleright p\right)=G(E, P)$, isto é, a álgebra gerada por energia e momento é um ideal; Como requerido, $[C(E, P), E]=[C(E, P), P]=$ $\left[C(E, P), \overline{X_{i}}\right]=0$, onde $C(E, p)=f^{2}(E) p^{2}-E^{2}$, isto é, $C(E, P)$ é um Casimir; Além disso, $\overline{X_{i}}$ é autoadjunto no espaço de Hilbert $\mathcal{H}^{\prime}$ das funções de $\mathbb{R}^{4}$ com produto interno:

$$
(f, g)=\int d \mu f^{*} g, \quad \int d \mu f=\int d^{4} p f \circ \Phi
$$

Poderíamos ter repetido o mesmo procedimento com $\Phi^{\prime}=\Phi \circ \mathcal{R}$, onde $\mathcal{R}\left(p^{0}, p^{j}\right)=$ $\left(p^{0}, R\left(f\left(p^{0}\right), \eta\left(p^{0}\right)\right) p^{j}\right)$, em que $R\left(\theta_{1}, \eta\right) \in S O(3)$ é uma rotação de um ângulo $\theta$ em torno da direção $\eta$ e $f\left(p^{0}\right)$ e $\eta\left(p^{0}\right)$ são um ângulo e uma direção como funções diferenciáveis de $p^{0}$, mostrando que $\mathfrak{p}_{\mathcal{N}}$ é não único. 
Capítulo 9

\section{Conclusões}

A questão fundamental abordada nesta tese é como o mecanismo de inflação pode ser modificado ao relaxarmos a hipótese relativística, isto é, de que a física é localmente invariante pela ação do grupo de Poincaré. Não abordamos essa questão na sua generalidade. m particular, existem linhas de pesquisa alternativas como Martin e Brandenberger (2001), Brandenberger (2003) e Martin e Brandenberger (2003), em que, na abordagem lagrangiana, a ação do campo escalar que dirige a inflação é modificada para levar em conta termos não relativísticos. Escolhemos como paradigma um mecanismo simples, porém inovador, de um modelo fenomenológico chamado de inflação não comutativa Alexander et al. (2003). Escolhemos esse modelo por quebrar o paradigma dominante na cosmologia, de que a inflação ocorre por força de uma fase dominada por campos escalares no universo primordial. Neste modelo, a componente dominante do universo primordial (segundo o modelo de concordância cósmica), a radiação, pode ela própria assumir um comportamento inflacionário se assumirmos que a relatividade especial deixa de ser válida em altas energias. Este modelo trazia consigo algo a mais, uma conjectura relacionada a outra ideia popular em desenvolvimentos da física de altas energias na última década: a ideia de espaço não comutativo. Conceito que, acredita-se, pode ser presente na física além do modelo padrão (Doplicher et al. (1995) e Connes et al. (1998)) e que foi relacionado a outros problemas em cosmologia, como a origem dos campos magnéticos de larga escala Bamba e Yokoyama (2004) e o problema da constante cosmológica Garattini e Nicolini (2011). A conjectura é que a natureza não comutativa do espaço, possivelmente comum a muitos modelos de física de altas energias, poderia ter como consequência indireta a inflação, segundo o mecanismo proposto, se a não comutatividade fosse modelada efetivamente numa mudança da relação 
entre energia e momento dos fótons. Se tal expectativa se confirmaria e de maneira tal que confirmasse as equações do modelo, era um problema não trivial.

Esta conjectura nos levou a contemplar a maneira usual como a física procura codificar suas hipóteses, tal como a ideia de espaço-tempo não comutativo, em lagragianas cada vez mais gerais. Como discutimos, a prescrição para tal modificação, no tocante à ideia de espaço não comutativo, é um ponto controverso na literatura. A prescrição usual, Douglas e Nekrasov (2001), também discutida nesta tese, é demonstrada, na cosmologia, ao menos no caso canônico, o mais estudado, arruinar o mecanismo padrão de inflação Palma e Patil (2009). Mas, como também discutido nesta tese, existem alternativas. Como saber qual a maneira correta de implementar o princípio? Em última análise, uma teoria física é melhor quando em melhor acordo com o experimento, não importando de que maneira escolhemos codificar hipóteses, mas sim as previsões finais. Na ausência de experimentos discriminantes, é um problema bem definido determinar se as prescrições disponíveis na literatura, ou novas propostas, para formular ou modificar a física estabelecida em função da informação que define o espaço não comutativo, predizem um tensor energia-momento efetivo compatível com o que postulam os autores da inflação não comutativa. Como modelo fenomenológico, a inflação não comutativa é uma modificação pontual na física. Por pontual, queremos dizer que afeta apenas a função de partição da radiação na descrição de teoria livre, não sendo uma regra geral para modificar a formulação da física como um todo, de acordo com suas premissas alternativas, e que tenha como consequência uma particular dinâmica para a radiação no universo primordial. Além disso, é uma modificação que não se dá em função da informação que define o espaço não comutativo, como desejavam os autores originais. Isso nos leva a uma restrição adicional do problema: Existe uma prescrição para modificar a física quântica local e relativística em função da informação que define o espaço não comutativo e que prediga o comportamento inflacionário da radiação segundo o mecanismo da inflação não comutativa, recuperando ainda a física usual em algum limite (baixas energias)? Este passou a ser o problema desta tese.

Analisar a ideia de espaço não comutativo, segundo originalmente concebida já nos trabalhos de Von Neumann Von Neumann (1996), em busca de uma solução para problema posto, levou esta tese por um interlúdio através da álgebra. Este interlúdio naturalmente levou a uma mudança de paradigma. Ao invés de operar com os métodos usuais da física, 
nomeadamente a técnica lagrangiana, operamos com os métodos da matemática pura. Ao invés de buscar uma nova regra para modificar lagrangianas para posterior quantização por métodos usuais, partimos de uma abordagem para a física quântica melhor adaptável à aplicação dos métodos da álgebra, a prescrição de Wigner, de que a física quântica relativística é definida não pela quantização de uma ação, mas pela identificação de uma particular representação do grupo de Poincaré. Sendo o grupo de Poincaré uma estrutura algébrica e o paradigma fundamental da inflação não comutativa que o caráter não relativístico dirige a inflação, o problema desta tese se reformula adicionalmente: Existe uma regra para definir uma deformação da estrutura algébrica do grupo de Poincaré em função da informação que define o espaço não comutativo seguida de uma prescrição para deformar teorias quânticas relativísticas locais segundo representações dessa estrutura algébrica e que levem às equações da inflação não comutativa?

Nesse momento, ao nos referirmos a espaços não comutativos, pode-se imaginar que estamos excluindo toda uma variedade de modificações não relativísticas da física que não se dão em função desta informação, mas que poderiam servir ao mesmo propósito de realizar a inflação segundo o mecanismo mencionado. Argumentamos, contudo, que a ideia de espaço não comutativo se apresenta na forma de uma linguagem algébrica que contém como caso particular a noção usual de espaço topológico empregada em física, sendo uma maneira diferente de codificar a informação deste. Por tanto, determinar a física como função de um grupo que atue como transformações num espaço possivelmente não comutativo é mais geral do que determinar a física como função de um grupo que atue como transformações no espaço topológico usual da física. Além disso, o estudo da estrutura matemática que define o espaço não comutativo, a $C^{*}$-álgebra, demonstra que há muito em comum entre as estruturas da álgebra, de modo que os mesmos métodos matemáticos aplicados nas $C^{*}$-álgebras são úteis nos grupos, álgebras de Lie e além, estabelecendo uma conexão a priori entre deformações da simetria relativística e espaços não comutativos.

Especificar a informação que define a $C^{*}$-álgebra não implica a existência de um grupo único que nele pode atuar. O que podemos fazer é nos perguntar como um grupo que atue num espaço não comutativo pode "parecer" o grupo de Poincaré quando não pudéssemos perceber a natureza não comutativa do espaço. Isso nos põe um problema semelhante àquele de quantizar uma teoria clássica, em que uma teoria quântica é requerida recupe- 
rar o comportamento clássico em algum limite, De certo modo, quantizar um grupo. Do mesmo modo como "quantizar" pode ser definido como mapear uma estrutura algébrica de observáveis em outra, mais geral, como discutido nesta tese, quantizar um grupo seria modificar sua estrutura algébrica. A prescrição genérica para tal não é ainda totalmente formulada. Argumentamos, contudo, que existem sugestões na literatura de que uma estrutura algébrica suficientemente geral para codificar numa única construção vários conceitos ligados a grupos, tais como a álgebra de Lie, o próprio grupo e ainda transformações não inversíveis em geral, poderia ser a outra estrutura algébrica mais geral. Esta é a chamada Hopf álgebra. Descrevemos um procedimento proposto na literatura para fazer essa passagem num caso particular.

Diante da falta de um regra geral $\mathcal{Q}_{\mathfrak{g}}$ para levar à informação que define o grupo de Poincaré, $\mathcal{P}$, e à informação que define o espaço não comutativo, $\mathcal{A}$, numa Hopf álgebra $H$, $\mathcal{Q}_{\mathfrak{g}}:(\mathcal{P}, \mathcal{A}) \rightarrow H$, de modo a assegurar um limite que poderia ser chamado "semiclássico", apenas postulamos que esta regra existe, ou melhor, postulamos que a física é invariante pela ação de uma Hopf álgebra. Ou melhor, invariante pela ação um grupo $\mathcal{P}_{\mathcal{N}}$ construído a partir de uma Hopf álgebra $\mathfrak{p}_{\mathcal{N}}$ que substitui a álgebra de Lie de Poincaré. Uma hipótese que se demonstra de grande generalidade, visto que as Hopf álgebras contém as Lie álgebras, como as do grupo de Poincaré, como casos particulares. Isso assegura uma estrutura ao grupo já compatível com o conceito de simetria quântica e permite definir as observáveis da teoria em função do grupo, isto se certas restrições à estrutura algébrica de $\mathfrak{p}_{\mathcal{N}}$ forem observadas. Discutimos também todo um conjunto de requerimentos sobre tal estrutura algébrica que permitem não apenas que as representações de $\mathcal{P}_{\mathcal{N}}$ repliquem as equações da inflação não comutativa, mas também levem a uma "física bem definida". Os autores que determinaram a Hopf álgebra associada ao particular exemplo de espaço não comutativo propuseram ainda outra maneira de modificar a ação dos campos, apenas no caso do campo escalar, de modo a definir a física, sem contudo se preocupar com o problema de quantizar essa ação. É sabido, contudo, que nem toda ação pode ser quantizada levando a uma teoria quântica bem definida no espaço de Hilbert. Em particular, a prescrição usual para modificação da ação de campos e definir as teorias não comutativas pode levar a problemas na renormalização Gurau et al. (2006) e unitariedade Rim e Yee (2003).

Seguimos um caminho alternativo: decidimos definir a teoria quântica construindo re- 
presentações da Hopf álgebra segundo a prescrição de Wigner para simetrias quânticas. Buscamos uma técnica que é originária da matemática das $C^{*}$-álgebras, mas que é de fundamental importância tanto na física quanto na matemática, chamada construção GNS. Essa técnica está envolvida na demonstração do teorema de Von Neumann, que diz que todas as representações irredutíveis da álgebra de Heisenberg são unitariamente equivalentes, por exemplo. Propusemos uma primeira versão da regra para definir a teoria física a partir da construção GNS aplicada a $\mathfrak{p}_{\mathcal{N}}$, regra aplicável apenas a teorias livres, mas que replicava as equações da inflação não comutativa. Além do problema de como replicar a construção GNS para as Hopf álgebras, restava ainda a dificuldade de definir a representação de $\mathcal{P}_{\mathcal{N}}$ que descreveria a física associada à radiação e a todos os demais campos relativísticos, um problema altamente não trivial, visto que existe uma infinidade não enumerável de representações inequivalentes. Propostas para tal são os principais resultados desta tese.

Percebemos, contudo, e este é um resultado original desta tese, que precisávamos considerar dois tipos distintos de representações do grupo $\mathcal{P}_{\mathcal{N}}$, que resultavam serem iguais no caso de um grupo de Lie, mas que poderiam ser diferentes no caso geral: as que chamamos de representações $\pi_{\mathfrak{g}}$, que não realizam a mesma álgebra de geradores $\mathfrak{p}_{\mathcal{N}}$, e as representações $\pi_{\mathcal{A}}$, que não apenas são representações do grupo $\mathcal{P}_{\mathcal{N}}$, mas que realizam a mesma álgebra $\mathfrak{p}_{\mathcal{N}}$. A formulação da física envolvendo representações $\pi_{\mathfrak{g}}$ é potencialmente problemática. Pode implicar que a noção de estado estável, que previne que estruturas como as átomos colapsem, não seria um conceito independente de referencial, ou que uma matriz de espalhamento conectando estados de uma partícula com estados de duas ou mais não seria compatível com conservação da energia em todos os referenciais. Em particular, uma física que postula que os geradores de simetrias satisfazem a álgebra $\mathfrak{p}_{\mathcal{N}}$, como a citada regra para mapear espaços não comutativos em Hopf álgebras, só pode ser definida por representações $\pi_{\mathcal{A}}$.

Encontramos que, sob requerimentos mínimos, não existe grupo $\mathcal{P}_{\mathcal{N}}$ cuja representação replique as equações da inflação não comutativa e que não seja uma representação do tipo $\pi_{\mathfrak{g}}$. Em particular, não existe grupo de Lie que realize a inflação não comutativa.

Para evitar problemas envolvendo a representação $\pi_{\mathfrak{g}}$, podemos mudar a regra que realiza a física a partir de representações de $\mathcal{P}_{\mathcal{N}}$ postulando que apenas representações do tipo $\pi_{\mathcal{A}}$ ocorrem na natureza. Postulados restringido a ocorrência de representações do grupo 
de Poincaré em favor de uma energia positiva definida fazem parte do modelo padrão. Propusemos uma prescrição alternativa baseado apenas em representações do tipo $\pi_{\mathcal{A}}$, que levam a uma noção única de relação de dispersão no universo, e que dessa vez era aplicável não apenas a teorias livres, mas a todo o universo de teorias de campo relativísticas. Mostramos que a referida prescrição não introduz infinitos adicionais na teoria, evitando por construção problemas com renormalização e unitariedade, que atormentam a quantização de lagrangianas genéricas. Chamamos esta prescrição de Prescrição $\pi_{\mathcal{A}}$.

A Prescrição $\pi_{\mathcal{A}}$ sugere que devemos modificar as equações da inflação não comutativa, e por consequência reabrir a questão da existência de classes de relações de dispersão inflacionárias, isto é, a existência de relações de dispersão e estados de equilíbrio termodinâmico que levariam à inflação. Do contrário, teríamos um problema de ajuste fino de condições iniciais para produzir inflação. Isso porque, apesar de que o mesmo princípio que leva à pressão negativa nas equações originais poder funcionar nas novas equações, existe a questão da duração da inflação e da saída graciosa, discutida em nosso trabalho para as equações originais. As equações da versão $\pi_{\mathcal{A}}$ da inflação ainda precisam ser obtidas, mas são analiticamente tratáveis e estamos trabalhando nisso.

Toda essa discussão seria inválida, se demonstrássemos que não existe estrutura algébrica como $\mathfrak{p}_{\mathcal{N}}$ que permita tal realização. Demonstramos, contudo, que existe e não é única uma álgebra $\mathfrak{p}_{\mathcal{N}}$ que permita este processo de construção da física. Não demonstramos contudo, que este pode estar relacionado a algum espaço não comutativo.

Esta tese é baseada no nosso trabalho publicado Machado e Opher (2012), mas possui muitos refinamentos e conclusões adicionais que modificaram a estrutura dos resultados em relação ao que publicamos. Originalmente a prescrição $\pi_{\mathcal{A}}$ foi proposta em nosso trabalho como um complemento aplicável à classe de teorias interagentes, isto é, um complemento da representação que descreve a teoria livre e leva à equação de estado da inflação não comutativa. Descobrimos depois que são prescrições necessariamente inequivalentes e que existe um problema conceitual na representação que leva à referida equação de estado. Nossos novos desenvolvimentos estão sendo transcritos e serão submetidos em breve, mas já estão nesta tese.

Observe que não abordamos a questão da geração de perturbações no modelo, e não existe mecanismo de inflação bem sucedido sem um mecanismo para gerar perturbações 
de densidade primordiais. Este mecanismo foi posteriormente desenvolvido pelos próprios autores do modelo em Koh e Brandenberger (2007). Uma ideia aplicável a qualquer fluido perfeito em equilíbrio termodinâmico local com equação de estado inflacionária. A ideia é que flutuações térmicas geram as sementes de estrutura. A estratégia é construir variáveis invariantes de calibre apenas em função da perturbações da métrica e do tensor energia momento de um fluido perfeito (a radiação não comutativa), partindo das equações 4.43 . 4.45, sob a condição 4.54, válida para um fluido perfeito, levando a seguinte equação para o componente $k$ do campo $\Phi$ :

$$
\Phi^{\prime \prime}+3 \mathcal{H}\left(1+c_{s}^{2}\right) \Phi^{\prime}+c_{s}^{2} k^{2} \Phi+\left(2 \mathcal{H}^{\prime}+\left(1+3 c_{s}^{2}\right) \mathcal{H}^{2}\right) \Phi=0
$$

do modo análogo ao descrito no capítulo 4, constrói-se um formalismo Hamiltoniano que recupere 4.54 em termos da variável $v$, definida por $\Phi=4 \pi G \sqrt{\rho+\mathcal{P}} \frac{z}{k^{2} c_{s}}\left(\frac{v}{z}\right)^{\prime}$, sendo $z$ o mesmo da eq. 4.76. Ao operador número de ocupação associado $\hat{N}=a_{k}^{*} a_{k}$ é atribuída um número de ocupação do tipo Bose-Einstein no momento em que a escala cruza, não o horizonte, mas o comprimento de correlação térmica $T^{-1}(\overline{\text { Koh e Brandenberger }}(2007))$. A amplitude de perturbação então evolui até atingir o horizonte sonoro, quando então congela (segundo o mesmo mecanismo que opera nas flutuações quânticas). O resultado é um espectro invariante de escala e gaussiano cuja amplitude depende da densidade e da temperatura em que ocorre a inflação.

Vale ressaltar que a nossa descrição de espaços não comutativos é diferente da geometria não comutativa, que se utiliza de um objeto chamado de tripla espectral Chamseddine et al. (2006). Algumas referências para a fenomenologia associada a essa abordagem são Nelson e Sakellariadou (2009), Marcolli et al. (2012), Nelson e Sakellariadou (2010) e Nelson et al. (2010). Na tripla espectral, não apenas a topologia é codificada em álgebra, mas um campo métrico. Estruturas adicionais são dadas à $C^{*}$-álgebra para cumprir esse propósito. A tripla espectral é, portanto, uma generalização do conceito de $C^{*}$-álgebra para codificar espaço curvo. 


\section{Referências Bibliográficas}

Landau L. D., Lifshitz E. M., The Classical Theory of Fields, Fourth Edition: Volume 2. Butterworth-Heinemann; 4 edition, 1980, 402 p.

Agostini A., Amelino-Camelia G., D'Andrea F., Hopf-Algebra Description of Noncommutative-Space Symmetries, International Journal of Modern Physics A, 2004, vol. 19 , p. 5187

Albrecht A., Magueijo J., Time varying speed of light as a solution to cosmological puzzles, Phys. Rev. D, 1999, vol. 59, p. 043516

Alexander S., Brandenberger R., Magueijo J., Noncommutative inflation, Phys. Rev. D, 2003, vol. 67, p. 081301

Alexander S. H. S., Magueijo J., Non-commutative geometry as a realization of varying speed of light cosmology, ArXiv High Energy Physics - Theory e-prints, 2001

Amelino-Camelia G., Arzano M., Coproduct and star product in field theories on Liealgebra noncommutative space-times, Phys. Rev. D, 2002, vol. 65, p. 084044

Aschieri P., Lectures on Hopf Algebras, Quantum Groups and Twists, ArXiv High Energy Physics - Theory e-prints, 2007

Balachandran A. P., Trahern G. C., Lectures on Group Theory for Physicists (Monographs and Textbooks in Physical Science). Humanities Pr; illustrated edition, 1986, 405 p.

Bamba K., Yokoyama J., Large-scale magnetic fields from dilaton inflation in noncommutative spacetime, Phys. Rev. D, 2004, vol. 70, p. 083508 
Banerjee R., Chakraborty B., Kumar K., Noncommutative gauge theories and Lorentz symmetry, Phys. Rev. D, 2004, vol. 70, p. 125004

Bogolubov N. N., Logunov A. A., Todorov I. T., Fulling S. A. T., Popova L. G. T., Introduction to Axiomatic Quantum Field Theory (Mathematical Physics Monograph Series, 18). W.A. Benjamin, Inc., 1975, 707 p.

Brandenberger R. H., Inflation and the Theory of Cosmological Perturbations. In High Energy Physics and Cosmology, 1997 Summer School , 1998, p. 412

Brandenberger R. H., Inflationary Cosmology: Progress and Problems, ArXiv High Energy Physics - Phenomenology e-prints, 1999

Brandenberger R. H., A Status Review of Inflationary Cosmology, ArXiv High Energy Physics - Phenomenology e-prints, 2001

Brandenberger R. H., Principles, Progress and Problems in Inflationary Cosmology, ArXiv Astrophysics e-prints, 2002

Brandenberger R. H., Trans-Planckian Physics and Inflationary Cosmology. In Cosmology and Particle Astrophysics (CosPA 02), 2003, p. 100

Brandenberger R. H., Conceptual Problems of Inflationary Cosmology and a New Approach to Cosmological Structure Formation. In Inflationary Cosmology, vol. 738 of Lecture Notes in Physics, Berlin Springer Verlag, 2008, p. 393

Carmona J. M., Cortés J. L., Gamboa J., Méndez F., Noncommutativity in field space and Lorentz invariance violation, Physics Letters B, 2003, vol. 565, p. 222

Chamseddine A. H., Connes A., Marcolli M., Gravity and the standard model with neutrino mixing, ArXiv High Energy Physics - Theory e-prints, 2006

Connes A., Douglas M. R., Schwarz A., Noncommutative geometry and Matrix theory, Journal of High Energy Physics, 1998, vol. 2, p. 3

de Faria E., de Melo W., Mathematical Aspects of Quantum Field Theory (Cambridge Studies in Advanced Mathematics) . Cambridge, UK: Cambridge University Press, 2010, $312 \mathrm{p}$. 
de Felice F., Clarke C. J. S., Relativity on Curved Manifolds. Cambridge, UK: Cambridge University Press, 1990, 459 p.

Debnath L., Mikusinski P., Introduction to Hilbert Spaces with Applications, Third Edition. Academic Press; 3 edition, 2005, 600 p.

do Carmo M. P., Geometria Riemanniana. Birkhäuser Boston, (1992)a, 300 p.

do Carmo M. P., Riemannian Geometry . Birkhäuser; 1 edition, 1992b, 320 pages

do Carmo M. P., Geometria diferencial de curvas e superfícies. Rio de Janeiro, Sociedade Brasileira de Matemática; 2 edition, 2005, 605 p.

Doplicher S., Fredenhagen K., Roberts J. E., The quantum structure of spacetime at the Planck scale and quantum fields, Communications in Mathematical Physics, 1995, vol. 172 , p. 187

Douglas M. R., Nekrasov N. A., Noncommutative field theory, Reviews of Modern Physics, 2001, vol. 73 , p. 977

Friedlander G., Joshi M., Introduction to the theory od distributions. Cambridge, UK: Cambridge University Press, 2 edition, 1998, 119 p.

Gamboa J., Loewe M., Rojas J. C., Noncommutative quantum mechanics, Phys. Rev. D, 2001, vol. 64, p. 067901

Garattini R., Nicolini P., Noncommutative approach to the cosmological constant problem, Phys. Rev. D, 2011, vol. 83, p. 064021

Gotay M. J., On the Groenewold Van Hove problem for R2n, JOURNAL OF MATHEMATICAL PHYSICS, NUMBER 4, 1999, vol. 40

Gurau R., Magnen J., Rivasseau V., Vignes-Tourneret F., Renormalization of NonCommutative $\Phi^{\wedge} 4 \_4$ Field Theory in x Space, Communications in Mathematical Physics, 2006, vol. 267 , p. 515

Haag R., Local Quantum Physics: Fields, Particles, Algebras (Theoretical and Mathematical Physics) . Springer; 2nd. rev. and enlarged ed. edition, 1996, 405 p. 
Hawking S., Ellis G., The large-scale structure of space-time.. Cambridge, UK: Cambridge University Press, 1973, $11+391$ p.

Heller M., Theoretical Foundations of Cosmology. New jersey, World Scientific Publishing, 1992, 147 p.

Isnard C., Introdução à medida e integração. IMPA, 2007, 314 p.

Koh S., Brandenberger R. H., Cosmological perturbations in non-commutative inflation, J. Cosmology Astropart. Phys., 2007, vol. 6, p. 21

Kolb E., Turner M., The Early Universe. Westview Press, 1994, 592 p.

Landsman N. P., Lecture notes on $\mathrm{C}^{*}$-algebras, Hilbert $\mathrm{C}^{*}$-modules, and quantum mechanics, ArXiv Mathematical Physics e-prints, 1998

Liddle A. R., An Introduction to Cosmological Inflation. In High Energy Physics and Cosmology, 1998 Summer School, 1999, p. 260

Linde A., Inflationary Cosmology. In Inflationary Cosmology, vol. 738 of Lecture Notes in Physics, Berlin Springer Verlag, 2008, p. 1

Loewner C., Theory of Continuous Groups (Dover Books on Mathematics). Dover Publications, 2008, 128 p.

Lyth D. H. D. H., Riotto A. A., Particle physics models of inflation and the cosmological density perturbation, Phys. Rep., 1999, vol. 314, p. 1

Machado U. D., Opher R., Generalized non-commutative inflation, Classical and Quantum Gravity, 2012, vol. 29, p. 065003

Majid S., Foundations of Quantum Group Theory. Cambridge, UK: Cambridge University Press, 2000, 664 p.

Marcolli M., Pierpaoli E., Teh K., The Coupling of Topology and Inflation in Noncommutative Cosmology, Communications in Mathematical Physics, 2012, vol. 309, p. 341

Martin J., Brandenberger R., Dependence of the spectra of fluctuations in inflationary cosmology on trans-Planckian physics, Phys. Rev. D, 2003, vol. 68, p. 063513 
Martin J., Brandenberger R. H., Trans-Planckian problem of inflationary cosmology, Phys. Rev. D, 2001, vol. 63, p. 123501

Mukhanov V., Cosmology and Astrophysics through Problems. Cambridge, UK: Cambridge University Press; First Published edition, 2005, 421 p.

Mukhanov V., Introduction to Quantum Effects in Gravity. Cambridge, UK: Cambridge University Press; First Published edition, 2007, 421 p.

Nakahara M., Geometry, Topology and Physics . New York, Taylor \& Francis Group; 2 edition, 2003, 320 pages

Nelson W., Ochoa J., Sakellariadou M., Constraining the Noncommutative Spectral Action via Astrophysical Observations, Physical Review Letters, 2010, vol. 105, p. 101602

Nelson W., Sakellariadou M., Inflation mechanism in asymptotic noncommutative geometry, Physics Letters B, 2009, vol. 680, p. 263

Nelson W., Sakellariadou M., Cosmology and the noncommutative approach to the standard model, Phys. Rev. D, 2010, vol. 81, p. 085038

Padmanabhan T., Cosmology and Astrophysics through Problems. Cambridge, UK: Cambridge University Press; First Published edition, 1996, 516 p.

Palma G. A., Patil S. P., UV/IR mode mixing and the CMB, Phys. Rev. D, 2009, vol. 80, p. 083010

Peter P., Uzan J. P., Primordial Cosmology. Oxford University Press, USA, 2009, 832 p.

Podles P., Muller E., Introduction to quantum groups. In eprint arXiv:q-alg/9704002 , 1997, p. 4002

Rim C., Yee J. H., Unitarity in space-time noncommutative field theories, Physics Letters B, 2003, vol. 574, p. 111

Sakurai J. J., Advanced Quantum Mechanics. Addison Wesley; 1 edition, 1967, 336 p.

Sattinger D. H., Weaver O. L., Lie Groups and Algebras with Applications to Physics, Geometry, and Mechanics (Applied Mathematical Sciences). Springer, 2010, 232 p. 
Schrödinger E., Space-Time Structure. Cambridge, UK: Cambridge University Press, 1950, $119 \mathrm{p}$.

Snyder H. S., Quantized Space-Time, Physical Review, 1947, vol. 71, p. 38

Spivak M., Calculus On Manifolds: A Modern Approach To Classical Theorems Of Advanced Calculus). Westview Press, 1971, 160 p.

Stewart J., Advanced General Relativity. Cambridge, UK: Cambridge University Press, 1993, $238 \mathrm{p}$.

Strocchi F., An Introduction to the Mathematical Structure of Quantum Mechanics: A Short Course for Mathematicians (Advanced Series in Mathematical Physics) . World Scientific Publishing Company; 2 edition, 2008, 292 p.

Szabo R. J., Quantum gravity, field theory and signatures of noncommutative spacetime, General Relativity and Gravitation, 2010, vol. 42, p. 1

Von Neumann J., Mathematical Foundations of Quantum Mechanics . Princeton University Press; Translation - from German edition, 1996, 464 p.

Wald R., General relativity. Chicago, University of Chicago Press, 1984, 504 p.

Weinberg S., Gravitation and Cosmology: Principles and Applications of the General Theory of Relativity. John Wiley \& Sons, Inc.; 1 edition, 1972, 688 p.

Weinberg S., The Quantum Theory of Fields, Volume 1: Foundations. Cambridge, UK: Cambridge University Press, 2005, 609 p.

Weinberg S., Cosmology. Oxford University Press, USA, 2008, 544 p.

Zainuddin H., Toh S. P., Nurisya M. S., Zainy M., Zulkarnain Z., Hassan J., Hassan Z. A., No-Go theorems and quantization, Journal of Fundamental Sciences., 2007, vol. 3, p. 127 